\title{
Experimental and Numerical Study of Flow Structures Associated with Low Aspect Ratio Elliptical Cavities
}

Taravat Khadivi, The University of Western Ontario

Supervisor: Dr. Eric Savory, The University of Western Ontario

A thesis submitted in partial fulfillment of the requirements for the Doctor of Philosophy degree in Mechanical and Materials Engineering

(C) Taravat Khadivi 2012

Follow this and additional works at: https://ir.lib.uwo.ca/etd

Part of the Aerodynamics and Fluid Mechanics Commons

\section{Recommended Citation}

Khadivi, Taravat, "Experimental and Numerical Study of Flow Structures Associated with Low Aspect Ratio Elliptical Cavities" (2012). Electronic Thesis and Dissertation Repository. 462.

https://ir.lib.uwo.ca/etd/462

This Dissertation/Thesis is brought to you for free and open access by Scholarship@Western. It has been accepted for inclusion in Electronic Thesis and Dissertation Repository by an authorized administrator of Scholarship@Western. For more information, please contact wlswadmin@uwo.ca. 


\section{EXPERIMENTAL AND NUMERICAL STUDY OF FLOW STRUCTURES ASSOCIATED WITH LOW ASPECT RATIO ELLIPTICAL CAVITIES}

(Spine title: FLOW STRUCTURES ASSOCIATED WITH ELLIPTICAL CAVITIES)

(Thesis format: Monograph)

by

Taravat Khadivi

Graduate Program in Mechanical and Materials Engineering

A thesis submitted in partial fulfillment of the requirements for the degree of

Doctor of Philosophy

The School of Graduate and Postdoctoral Studies

The University of Western Ontario

London, Ontario, Canada

(C) Taravat Khadivi 2012 
THE UNIVERSITY OF WESTERN ONTARIO

School of Graduate and Postdoctoral Studies

\section{CERTIFICATE OF EXAMINATION}

Supervisor

Examiners

Dr. Eric Savory

Dr. Girma Bitsuamlak

$\underline{\text { Supervisory Committee }}$

Dr. Roger E. Khayat

Dr. Gregory Kopp

Dr. Gary Rankin

Dr. Anthony Straatman

Dr. Jun Yang

The thesis by

\section{Taravat Khadivi}

entitled:

\section{Experimental and Numerical Study of Flow Structures Associated with Low Aspect Ratio Elliptical Cavities}

is accepted in partial fulfillment of the requirements for the degree of Doctor of Philosophy 


\section{Abstract}

The flow regimes associated with a 2:1 aspect ratio, elliptical planform cavity in a turbulent flat plate boundary layer have been systematically examined for various depth/width ratios $(0.1$ to 1.0$)$ and yaw angles $\left(0^{\circ}\right.$ to $\left.90^{\circ}\right)$, using a combination of wind tunnel experiments (involving Particle Image Velocimetry and helium bubble visualization) and Computational Fluid Dynamics (CFD) simulations (employing threedimensional steady calculations with the Reynolds Stress model turbulence closure). Satisfactory agreement has been found between the results using the two methods, indicating that the steady numerical simulations can be a cost-effective tool to predict the mean flow features.

The flow has been found to be highly dependent on yaw angle and cavity depth. For each of the three broad flow categories specified according to yaw angle, which include the symmetric flow regime (yaw angle $=0^{\circ}$ ), the straight vortex regime (yaw angle $=90^{\circ}$ ) and the asymmetric flow regime $\left(15^{\circ} \leq\right.$ yaw angle $\left.\leq 60^{\circ}\right)$ different regimes are found to exist, depending on cavity depth. For each combination of yaw angle and depth, the flow has been analyzed through investigation of shear layer parameters, the three-dimensional vortex structure, pressure distribution and drag, wake flow, and vortex oscillations.

While the elliptical cavity flows have been found to have some similarities with those of nominally two-dimensional and rectangular cavities, the three-dimensional effects due to the low aspect ratio and curvature of the walls give rise to features exclusive to low aspect ratio elliptical cavities, including formation of cellular structures at intermediate depths and unique vortex structures within and downstream of the cavity.

The three-dimensional flow structure of the flow is most pronounced in the asymmetric regimes with large yaw angles $\left(45^{\circ}\right.$ and $\left.60^{\circ}\right)$. The dominant feature in this regime is the formation of a trailing vortex that is associated with high drag and flow oscillations within the cavity. 


\section{Keywords}

Cavity, Elliptical Planform, Yaw Angle, Flow Structure, Separated Shear Layer, Vortex, Pressure Distribution, Drag, Turbulent Boundary Layer, Computational Fluid Dynamics (CFD), Particle Image Velocimetry (PIV). 


\section{Dedication}

This thesis is dedicated, in loving memory, to my father Iraj Khadivi, who passed away on July 28, 2011, while I was preparing this thesis. I know how important it was for him to see me graduate, but he never got the chance. He was my hero and role model. His beautiful heart was warm, nonjudgmental, and always open. I miss him every day of my life. 


\section{Acknowledgments}

It is my pleasure to thank the many people who made this thesis possible. My gratitude goes first and foremost to my advisor, Professor Eric Savory for his continued guidance, instruction, and encouragement. Also, I would like to thank him for his support and understanding during the difficult time I have been going through.

I am also grateful to Professor Gregory Kopp and Professor Horia Hangan for providing essential parts of the experimental equipment and facilities I have used in my research.

I want to acknowledge Mr. Christopher Vandelaar from University Machine Shop, whose professional services have been essential in preparation of my experimental setup. My sincere thanks go to Mr. Gerry Dafoe who was always willing to help and make equipment available at the Boundary Layer Wind Tunnel Laboratory.

I would like to thank my fellow graduate students, Mr. Adam Kirchhefer and Ms. Maryam Refan for helping me with the experimental setup and equipment, and also the members the Advanced Fluid Mechanics research group for their constructive comments.

My special appreciation goes to my loving, supportive, and patient husband, Dr. Arash Naghib-Lahouti, who has unconditionally supported me during my studies.

And finally, I would like to express my thanks to my late father Iraj, and my mother Vajiheh, for their encouragement and faith in me. I love them so much, and I would not have made it this far without them. 


\section{Table of Contents}

CERTIFICATE OF EXAMINATION ….................................................................. ii

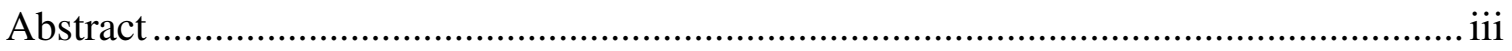

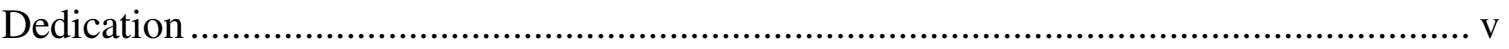

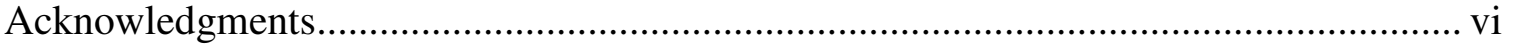

Table of Contents ......................................................................................................... vii

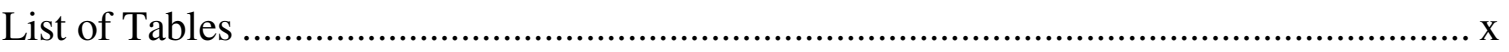

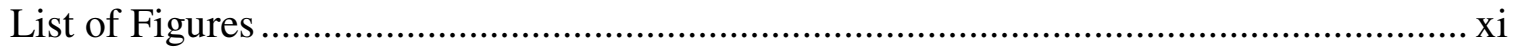

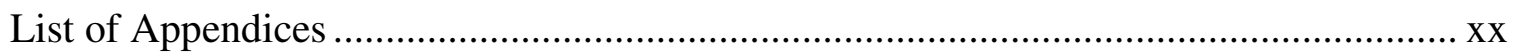

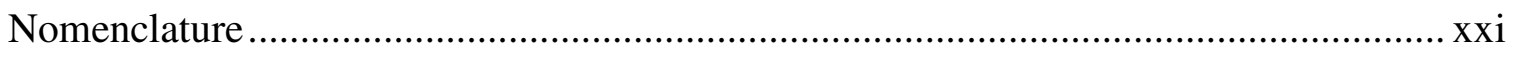

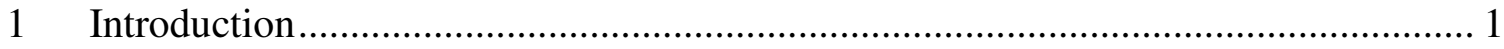

1.1 Gaps in Research on Elliptical Cavities .................................................. 2

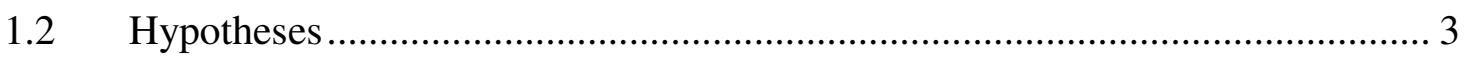

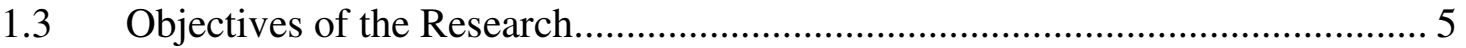

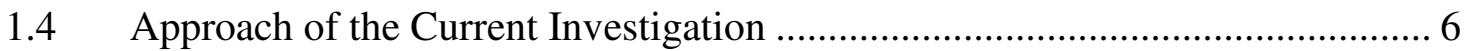

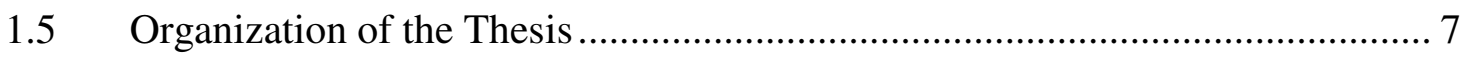

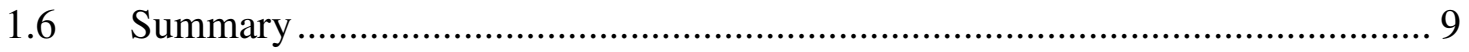

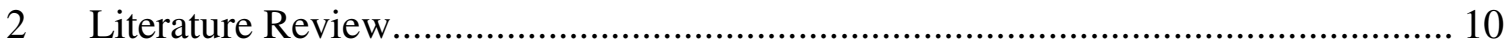

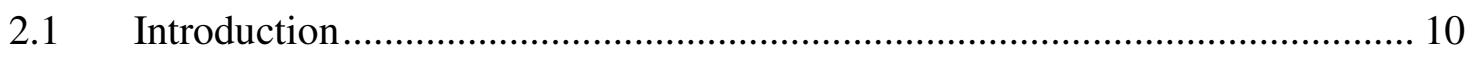

2.2 A Brief Review of the Fundamental Concepts of Vorticity ........................... 11

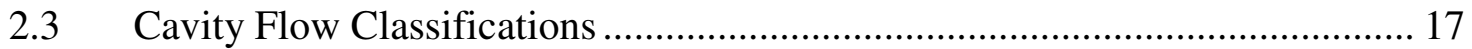

2.4 Effect of Upstream Conditions on Cavity Flow ........................................... 24

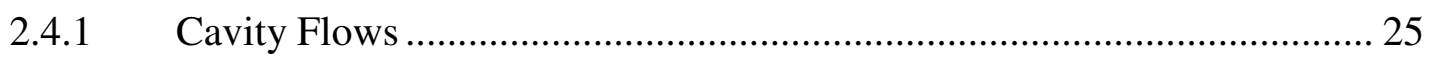

2.4.2 Backward Facing Step Flows............................................................... 29

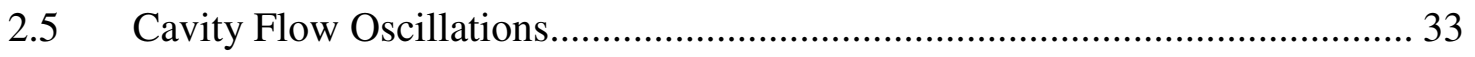

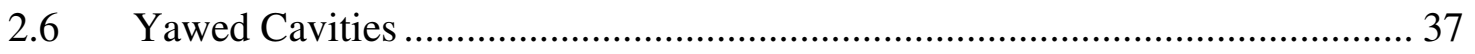

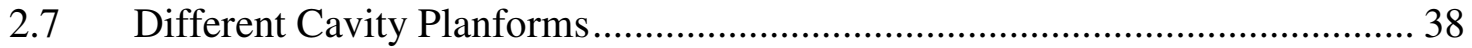

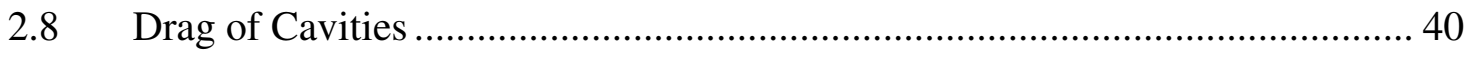

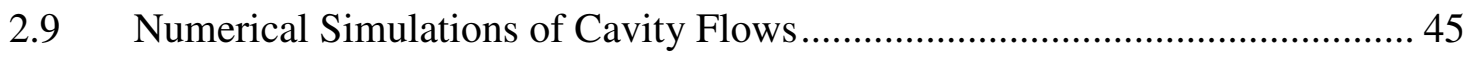

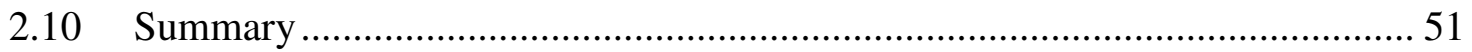




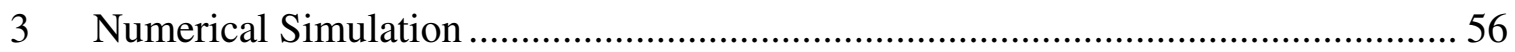

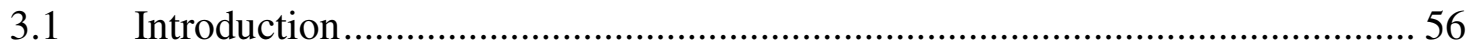

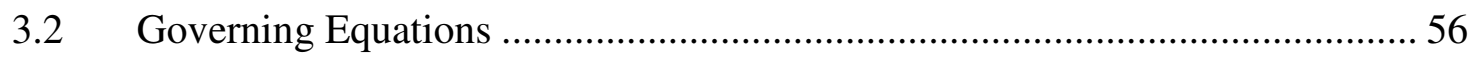

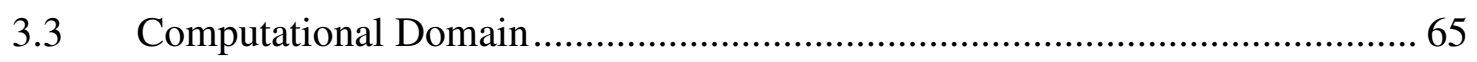

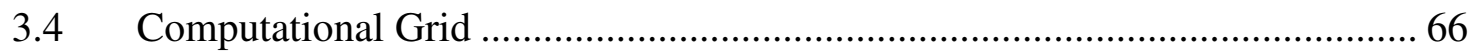

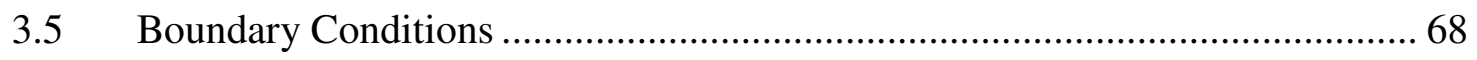

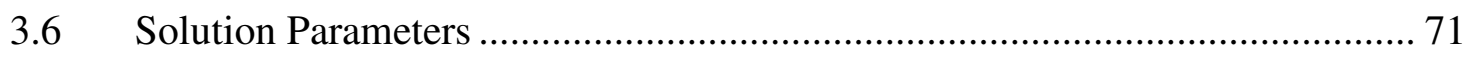

3.7 Validation of the Results of Numerical Simulations ....................................... 72

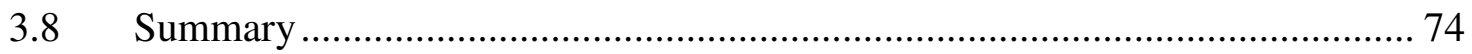

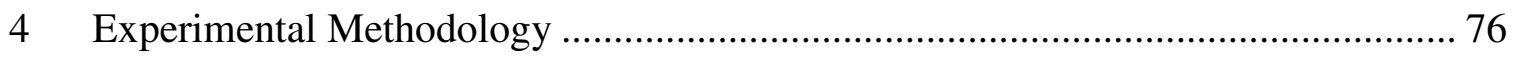

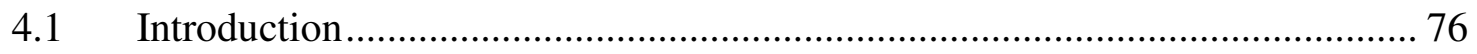

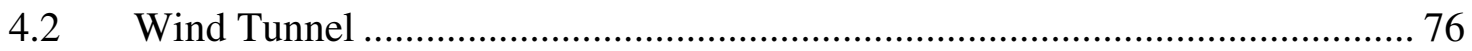

4.3 Model Specifications ................................................................................. 77

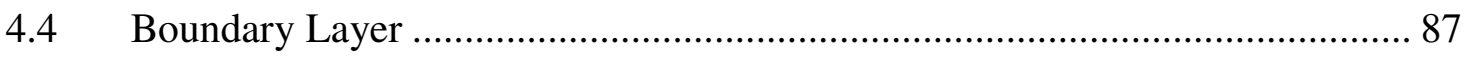

4.5 Pressure Measurement Equipment................................................................. 90

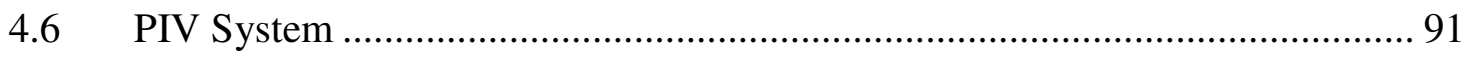

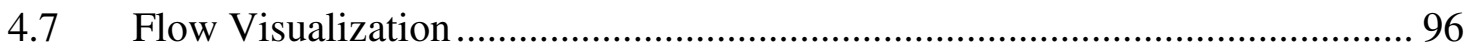

4.8 PIV Measurement Planes and Convergence Test ………………………......... 98

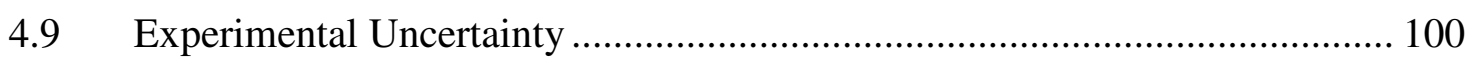

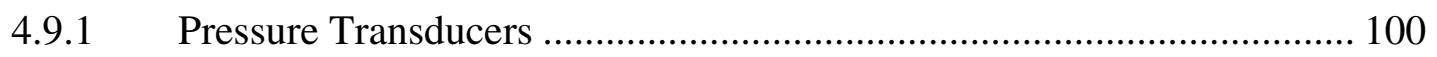

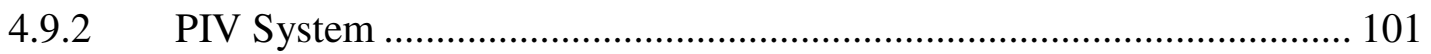

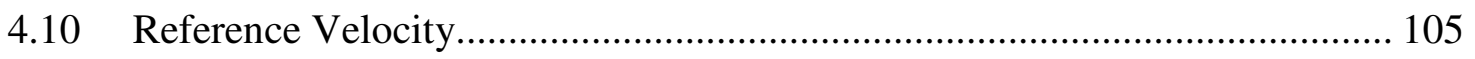

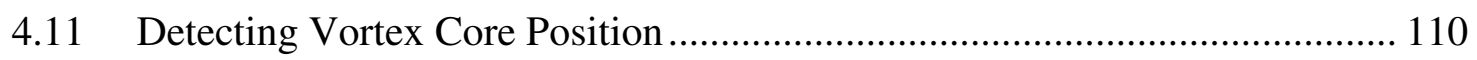

4.12 Proper Orthogonal Decomposition ................................................................ 112

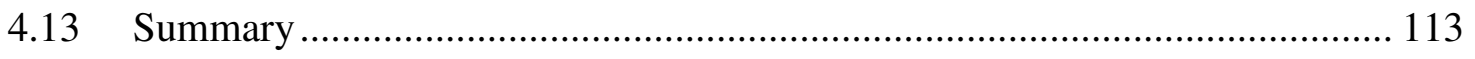

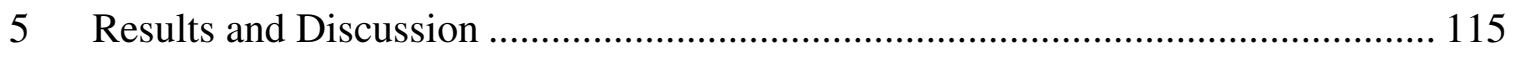

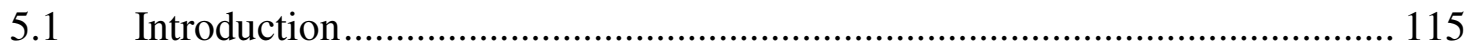

5.2 Comparative Evaluation of the Experimental and Numerical Results ........... 117

5.2.1 Comparison of PIV Experiments and Numerical Simulations ................ 118

5.2.2 Relationship between Pressure Distribution and Vortex Core Position . 124

$5.3 \quad$ Symmetric Flow Regimes.......................................................................... 130 
5.3.1 Behaviour of the Shear Layer …………………................................. 130

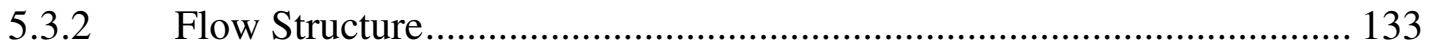

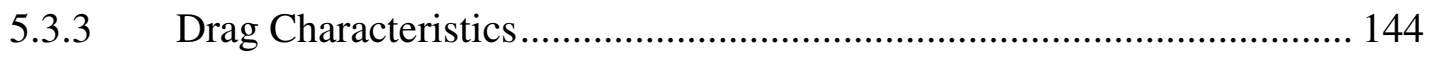

5.3.4 Flow Downstream of the Cavity ………………................................. 145

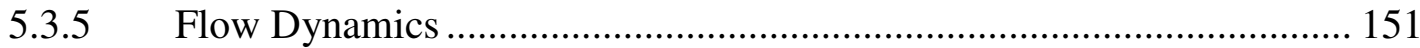

5.3.6 Summary of Findings for Symmetric Flow Regimes ............................. 160

$5.4 \quad$ Nominally Two-Dimensional Flow Regime.................................................... 163

5.4.1 Behaviour of the Shear Layer ............................................................. 163

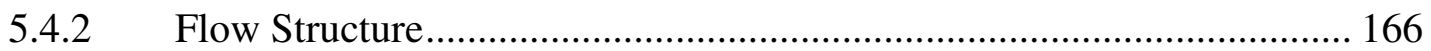

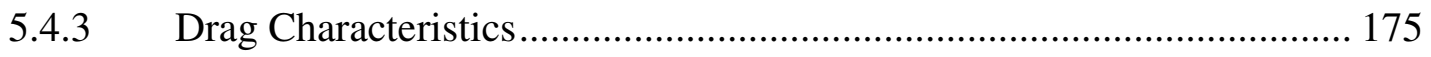

5.4.4 Flow Downstream of the Cavity ………………................................. 176

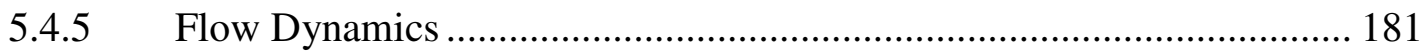

5.4.6 Summary of Findings for the Nominally Two-Dimensional Flow Regime 187

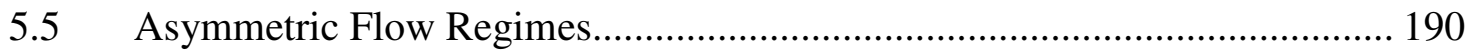

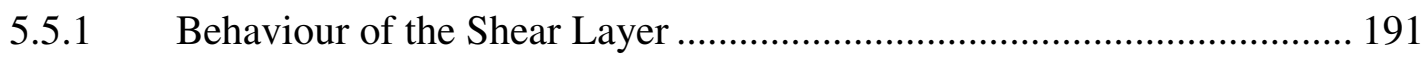

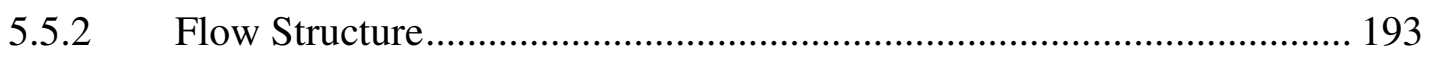

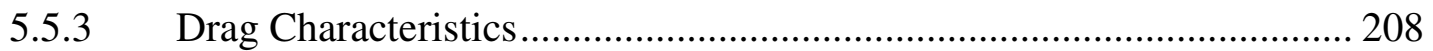

5.5.4 Flow Downstream of the Cavity ………………................................ 210

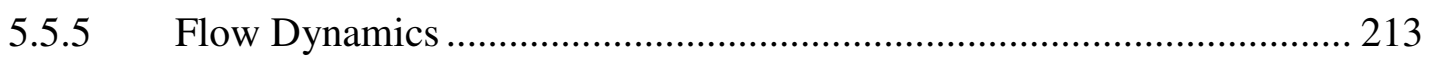

5.5.6 Summary of Findings for the Asymmetric Flow Regimes ..................... 219

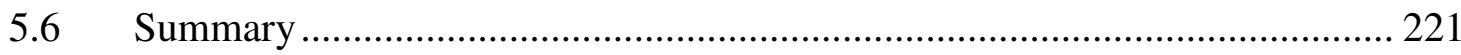

6 Conclusions and Recommendations ……………………................................ 224

$6.1 \quad$ Summary of the Findings............................................................................. 224

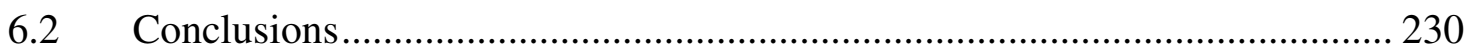

6.3 Recommendations for Future Work....................................................... 233

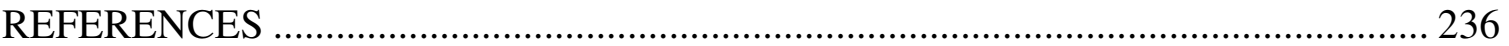

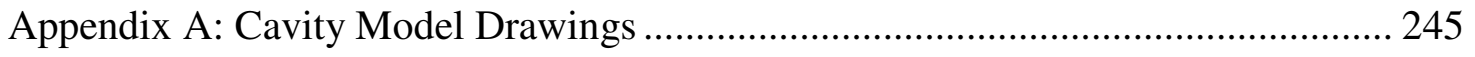

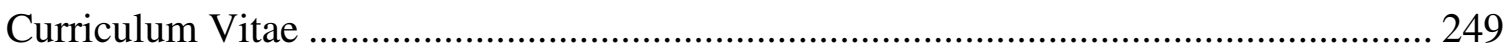




\section{List of Tables}

Table 3-1 Variations of selected parameters with the number of cells

Table 3-2 Quantitative comparison of $\mathrm{C}_{\mathrm{p}}$ values obtained by numerical simulations and

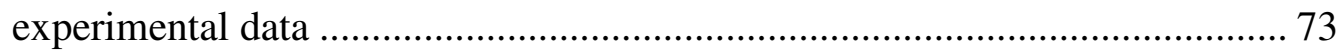

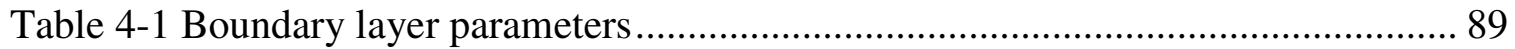

Table 4-2 The locations of vertical planes for different yaw angles............................. 98

Table 4-3 The locations of horizontal planes for different cavity depths ....................... 99

Table 4-4 Average variations of $\bar{u}$ and $u_{r m s}$ due to increasing the number of samples .. 100

Table 4-5 Total uncertainty of flow variables.......................................................... 104

Table 4-6 $\bar{u} / \mathrm{U}_{\text {inf }}$ for two yaw angles at two different locations $(\mathrm{h} / \mathrm{D}=1.0) \ldots \ldots \ldots \ldots \ldots . . . . . .109$

Table 4-7 List of numerical simulation and experimental measurement cases ............. 114

Table 4-8 Comparison of the parameters of present and Hering's experiments [58] .... 114

Table 5-1 Average distance between vortex cores (CFD \& PIV results) ....................... 124

Table 5-2 Amplitude of oscillations of the vortex core based on the phase-averaged

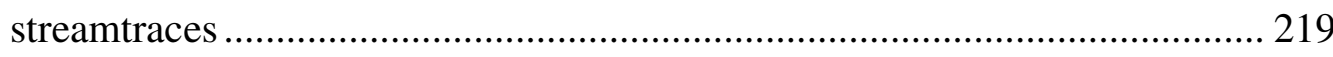




\section{List of Figures}

Figure 2-1 General cavity variables shown for a rectangular cavity ............................ 11

Figure 2-2 Closed (a) and open (b) cavity flow regimes at subsonic speeds (adapted from

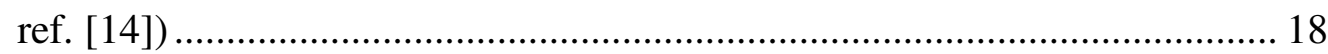

Figure 2-3 Closed (a) and open (b) cavity flow regimes at supersonic speeds (adapted

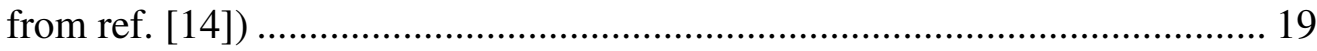

Figure 2-4 Cavity base centreline pressure profiles (adapted from ref. [15]).................. 20

Figure 2-5 Main three-dimensional flow structures for rectangular closed (a) and open (b) cavities (adapted from ref. [14]) .......................................................... 21

Figure 2-6 Topology of flow for closed (a) and open (b) rectangular cavities................ 24

Figure 2-7 Mean flow field (adapted from ref. [24]) ................................................. 28

Figure 2-8 Typical feedback resonance mechanism [53] …...................................... 35

Figure 2-9 Schematic model of aerodynamic phenomena resulting in vorticity shedding

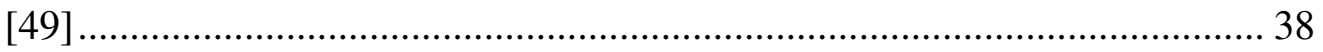

Figure 2-10 Resulting flow regimes of yawed elliptical cavities [6] ............................ 40

Figure 2-11 Variation of normalized drag coefficient with $\mathrm{h} / \mathrm{D}$ for rectangular cavities at $0^{\circ}$ yaw angle (The line is added to visualize the general trend.) .................. 43

Figure 2-12 Variation of normalized drag coefficient with $\mathrm{h} / \mathrm{D}$ for elliptical cavities at $0^{\circ}$ yaw angle (The lines are added to visualize the general trend for elliptical (solid line) and circular (dashed line) planform shape.) ............................. 44

Figure 2-13 Effect of yaw angle and depth on the normalized drag coefficient of an

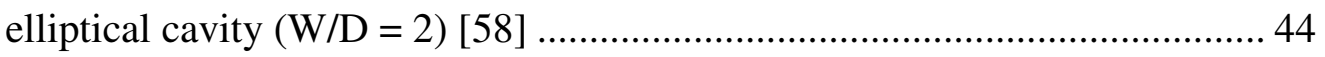

Figure 3-1 Typical velocity distribution in turbulent flow near a wall .......................... 61

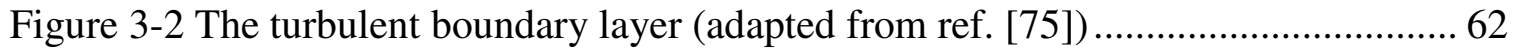

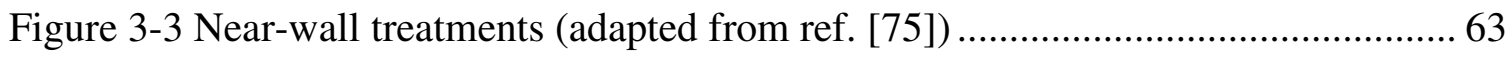

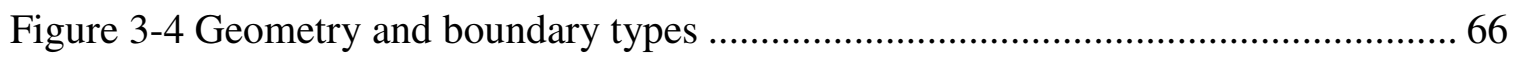

Figure 3-5 Computational grid in the cavity and the surroundings ............................... 67

Figure 3-6 Comparison of $C_{p}(\mathrm{a}), \bar{u} / U_{\mathrm{inf}}(\mathrm{b})$, and $\overline{u^{\prime} u^{\prime}} / U_{\mathrm{inf}}^{2}$ (c) for three different numbers of

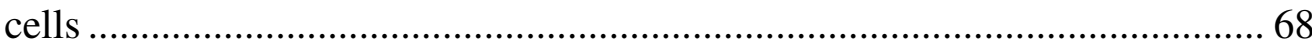

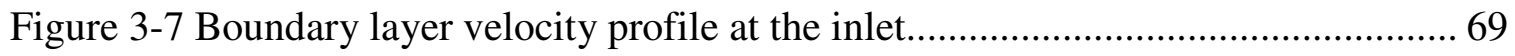


Figure 3-8 Dimensionless $\overline{u^{\prime} u^{\prime}}$ (a), $\overline{v^{\prime} v^{\prime}}$ (b), and $\overline{u^{\prime} v^{\prime}}$ (c) Reynolds stress profiles at the inlet 70

Figure 3-9 Turbulence kinetic energy $(k)$ (a) and Turbulence dissipation rate $(\varepsilon)(b)$ at the inlet 71

Figure 3-10 Comparison of $\mathrm{C}_{\mathrm{p}}$ profiles on the centreline of the cavity base for three different $h / D$ ratios at yaw angles of $0^{\circ}$ (a) and $90^{\circ}$ (b) ............................. 73

Figure $3-11 \mathrm{C}_{\mathrm{p}}$ contours on cavity walls and around the cavity for $\mathrm{h} / \mathrm{D}=1.0$ at $0^{\circ}$ yaw angle based on numerical simulation (a) and experimental results [58] (b) ... 74

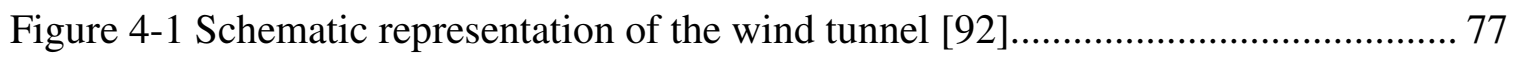

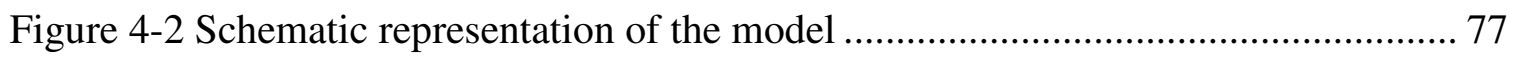

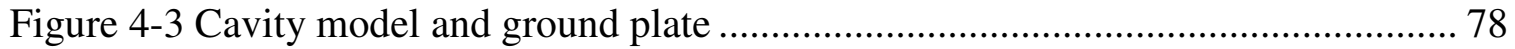

Figure 4-4 Leading edge shape and surface roughness ............................................. 79

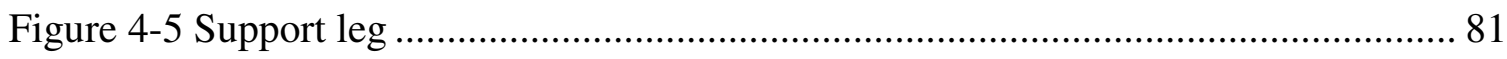

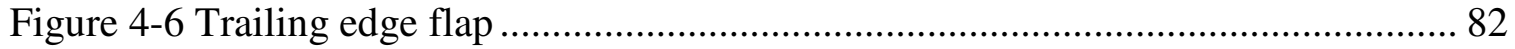

Figure 4-7 Plate surface pressure coefficient upstream of the cavity in streamwise (a) and spanwise directions (b) for different combinations of yaw angle and the flap

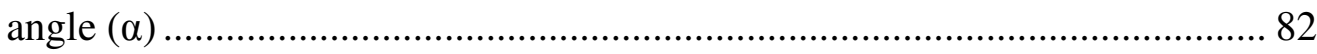

Figure 4-8 Velocity Profiles $1 \mathrm{~cm}$ upstream of the cavity lip...................................... 84

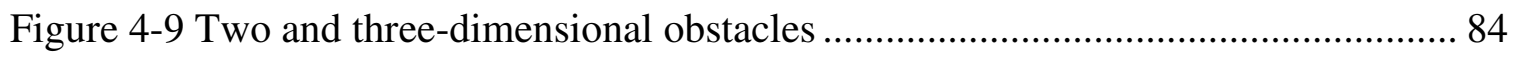

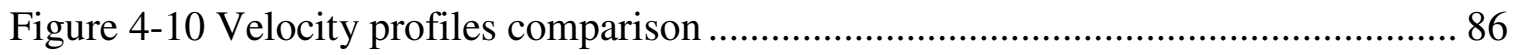

Figure 4-11 Velocity profiles downstream a two-dimensional obstacle, solid line shows experimental data and dashed line shows the power law profile [103] ......... 86

Figure 4-12 Comparison of the measured turbulent boundary layer data to the law of the wall. 88

Figure 4-13 Comparison of dimensionless $\bar{u}$ profiles (a) and $\overline{u^{\prime} v^{\prime}}$ profiles (b) at seven upstream spanwise locations. 89

Figure 4-14 Example calibration curves for pressure transducers 90

Figure 4-15 Images of the experimental setup, showing the main components: (1) model, (2) camera, (3) laser, (4) spherical lens, (5) laser beam reflector and cylindrical lens, (6) synchronizer, (7) imager recording computer ............... 93

Figure 4-16 schematic Laskin nozzle aerosol generator ........................................... 93 
Figure 4-17 Dry ice placed at the upstream of the cavity (a) and inside the cavity (b) .... 97

Figure 4-18 Helium bubble generator 98

Figure 4-19 Effect of the number of samples on distributions of $\bar{u}$ (a) and $u_{r m s}$ (b) on a horizontal line located at $\mathrm{z} / \mathrm{D}=0$ and $\mathrm{y} / \mathrm{D}=-0.6$, for the cavity with $\mathrm{h} / \mathrm{D}=1.0$

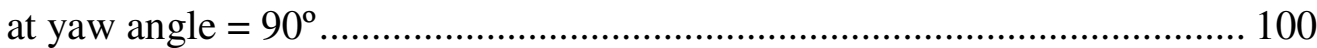

Figure 4-20 Image distortion for yaw angle $=90^{\circ}$ (a) and yaw angle $=0^{\circ}$ (b) $\ldots \ldots \ldots \ldots . . .105$

Figure 4-21 Velocity profile comparison for two different yaw angles at two different locations 107

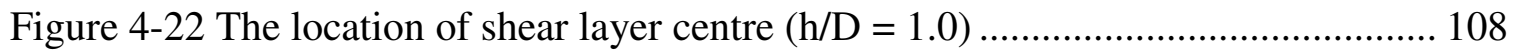

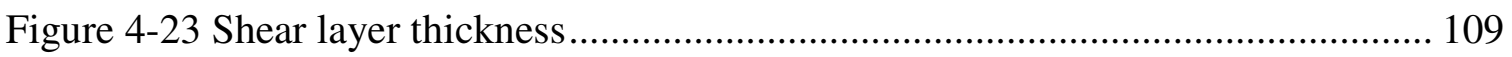

Figure 4-24 Schematic figure of velocity profile at $\mathrm{x} / \mathrm{D}=0$ (green line), contours of $\frac{\partial u}{\partial y}$ (red lines), shear layer (black dashed line), cavity walls (blue lines), and $U_{\text {ref }}$ (black vector) 110

Figure 4-25 Different methods to find vortex core location inside the cavity $(\mathrm{h} / \mathrm{D}=1.0 \&$ yaw $\left.=90^{\circ}\right)$ 111

Figure 5-1 Different flow regimes of yawed elliptical cavity (adapted from ref. [6]). Symmetric, asymmetric and nominally two-dimensional regimes are highlighted in blue, red, and green, respectively. .................................. 117

Figure 5-2 Numerical and experimental results comparison of $\bar{u}$ and $\overline{u^{\prime} u^{\prime}}$ profiles for $\mathrm{h} / \mathrm{D}$ $=1.0$ at $90^{\circ}$ yaw angle 120

Figure 5-3 Numerical and experimental results comparison of $\bar{u}$ and $\overline{u^{\prime} u^{\prime}}$ profiles for h/D $=1.0$ at $45^{\circ}$ yaw angle 121

Figure 5-4 Numerical and experimental results comparison of $\bar{u}$ and $\overline{u^{\prime} u^{\prime}}$ profiles for h/D $=1.0$ at $0^{\circ}$ yaw angle 122

Figure 5-5 Vortex core position (a) and pressure coefficient contours on cavity base, ground plane, and side walls for $\mathrm{h} / \mathrm{D}=1.0$, yaw angle $=0^{\circ}$ from reference [58] (b) and numerical simulations (c) 126

Figure 5-6 Vortex core position (a) and pressure coefficient contours on cavity base, ground plane, and side walls for $\mathrm{h} / \mathrm{D}=0.5$, yaw angle $=45^{\circ}$ from reference [58] (b) and numerical simulations (c) 128 
Figure 5-7 Vortex core position (a) and pressure coefficient contours on cavity base, ground plane, and side walls for $\mathrm{h} / \mathrm{D}=0.5$, yaw angle $=90^{\circ}$ from reference [58] (b) and numerical simulations (c)

Figure 5-8 Shear layer thickness at $0^{\circ}$ yaw angle 131

Figure 5-9 The location of shear layer centre at $0^{\circ}$ yaw angle. 132

Figure 5-10 Streamlines and $C_{p}$ contours for cavity with $h / D=1.0$ at $0^{\circ}$ yaw angle at $z / D$ $=0(\mathrm{a})$ and $\mathrm{z} / \mathrm{D}=0.7(\mathrm{~b})$

Figure 5-11 Instantaneous flow visualization image obtained using the Helium bubble technique, for the cavity with $\mathrm{h} / \mathrm{D}=1.0$ at yaw angle $=0^{\circ}$ 135

Figure 5-12 Vortex core positions and $C_{p}$ contours at yaw angle $=0^{\circ}$ for $h / D=0.7,0.5$, and 0.35

Figure 5-13 Sectional streamlines and $C_{p}$ contours for cavity with $h / D=0.5$ at $0^{\circ}$ yaw angle at $\mathrm{z} / \mathrm{D}=0$ (a) and $\mathrm{z} / \mathrm{D}=0.7$ (b) 138

Figure 5-14 $\mathrm{C}_{\mathrm{p}}$ contours for a rectangular cavity $(\mathrm{W} / \mathrm{D}=3.18 \& \mathrm{~h} / \mathrm{D}=0.5)$ at $0^{\circ}$ yaw angle (adapted from ref. [49]).

Figure 5-15 $C_{p}$ contours at yaw angle $=0^{\circ}$ for $h / D=0.5$, Hering [58] (a), Savory and Toy (adapted from ref. [5]) (b) 140

Figure 5-16 Vortex core positions and $C_{p}$ contours at yaw angle $=0^{\circ}$ for $h / D=0.2$ and 0.1 142

Figure 5-17 Streamlines and $C_{p}$ contours of cavity with $h / D=0.2$ at $0^{\circ}$ yaw angle (a) and instantaneous streamlines of a nominally two-dimensional rectangular cavity with $\mathrm{h} / \mathrm{D}=0.13$ [119] 142

Figure 5-18 Streamlines and and $C_{p}$ contours for cavity with $h / D=0.1$ at $0^{\circ}$ yaw angle at $\mathrm{z} / \mathrm{D}=0$ (a) and $\mathrm{z} / \mathrm{D}=0.7$ (b) 143

Figure $5-19 \mathrm{C}_{\mathrm{p}}$ contours at yaw angle $=0^{\circ}$ for $\mathrm{h} / \mathrm{D}=0.1[58]$ 144

Figure 5-20 Comparison of drag coefficient at yaw angle $=0^{\circ}$ (The lines are added to visualize the general trend for each planform shape.) 145

Figure 5-21 Velocity deficit contours based on numerical simulations (left) and experimental measurements [58] (right) at $0^{\circ}$ yaw angle

Figure 5-22 Velocity deficit contours based on PIV measurements at $0^{\circ}$ yaw angle in a horizontal plane. 149 
Figure 5-23 Quantitative comparison of velocity deficit at $0^{\circ}$ yaw angle 150

Figure 5-24 Streamwise vorticity contours at $\mathrm{x} / \mathrm{D}=0.5$ for $\mathrm{h} / \mathrm{D}=0.7$ (a) and $\mathrm{h} / \mathrm{D}=0.5$ (b) at $0^{\circ}$ yaw angle.

Figure 5-25 $\mathrm{u}_{\mathrm{rms}}$ contours for $\mathrm{h} / \mathrm{D}=1.0$ (a), 0.7 (b), and 0.5 (c) at $0^{\circ}$ yaw angle at $\mathrm{z} / \mathrm{D}=0$ 153

Figure 5-26 Comparison of $\mathrm{u}_{\mathrm{rms}}$ (a) and $\mathrm{v}_{\mathrm{rms}}$ (b) profiles on the vertical centreline 153

Figure 5-27 The first four POD modes of the fluctuating streamwise velocity component 154

Figure 5-28 The first four POD modes of the fluctuating vertical velocity component. 155 Figure 5-29 The first forty normalized eigenvalues for $\mathrm{h} / \mathrm{D}=1.0$ (a) and 0.5 (b) at $0^{\circ}$ yaw angle at $\mathrm{z} / \mathrm{D}=0$. 156

Figure 5-30 $\mathrm{v}_{\mathrm{rms}}$ contours on vertical mid-plane $(\mathrm{z} / \mathrm{D}=0)$ by Oszalp (adapted from ref. [29]) for a two-dimensional rectangular cavity (a), Haigermoser (adapted from ref. [54]) for a circular cavity (b), and the present study (c) 156

Figure 5-31 Normalized time-varying coefficients for the cavities with $\mathrm{h} / \mathrm{D}=1.0$ (a) and $0.5(\mathrm{~b})$ 157

Figure 5-32 Phase averaged streamtraces in the vertical plane located at $\mathrm{z} / \mathrm{D}=0.35$, for the cavity with $\mathrm{h} / \mathrm{D}=1.0$. The phase interval between consecutive plots is $90^{\circ}$.

Figure 5-33 Phase averaged streamtraces in the vertical plane located at $\mathrm{z} / \mathrm{D}=0.35$, for the cavity with $h / D=0.5$. The phase interval between consecutive plots is $90^{\circ}$.

Figure 5-34 Schematic representation of the flow structure in an elliptical cavity in the symmetric-deep flow regime..... 161

Figure 5-35 Schematic representation of the flow structure in an elliptical cavity in the symmetric-cellular structure flow regime. 161

Figure 5-36 Schematic representation of the flow structure in an elliptical cavity in the symmetric-shallow flow regime 162

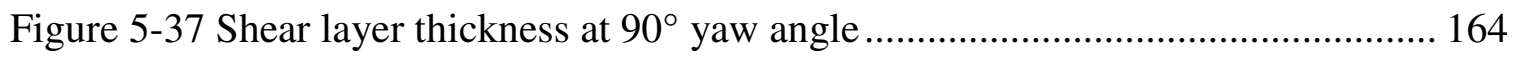

Figure 5-38 The location of shear layer centre at $90^{\circ}$ yaw angle 165 
Figure 5-39 Vortex core position (a) and pressure coefficient contours on cavity base, ground plane, and side walls for $\mathrm{h} / \mathrm{D}=1.0$, yaw angle $=90^{\circ}$ from reference [58] (b) and numerical simulations (c) 167

Figure 5-40 Sectional streamlines and $C_{p}$ contours for cavity with $h / D=1.0$ at $90^{\circ}$ yaw angle at $\mathrm{z} / \mathrm{D}=0(\mathrm{a})$ and $\mathrm{z} / \mathrm{D}=0.35(\mathrm{~b})$ 168

Figure 5-41 Example of mean velocity contours and streamlines in a nominally twodimensional rectangular cavity with $\mathrm{h} / \mathrm{D}=0.5$ (adapted from ref. [122]).... 168

Figure 5-42 Instantaneous flow visualization images obtained using the Helium bubble technique, for the cavity with $\mathrm{h} / \mathrm{D}=1.0$ at yaw angle $=90^{\circ}$ 169

Figure 5-43 Streamlines for cavity with $\mathrm{h} / \mathrm{D}=0.5$ at $90^{\circ}$ yaw angle at $\mathrm{z} / \mathrm{D}=0$ (a) and $\mathrm{z} / \mathrm{D}$ $=0.35(\mathrm{~b})$ 170

Figure 5-44 streamlines of a nominally two-dimensional rectangular cavity $(\mathrm{h} / \mathrm{D}=0.25)$ (adapted from ref. [24])...... 171

Figure 5-45 $\mathrm{C}_{\mathrm{p}}$ contours for a rectangular cavity $(\mathrm{W} / \mathrm{D}=2 \& \mathrm{~h} / \mathrm{D}=0.5)$ at $0^{\circ}$ yaw angle (adapted from ref. [49]) 171

Figure 5-46 Vortex core position (a) and pressure coefficient contours on cavity base, ground plane, and side walls for $\mathrm{h} / \mathrm{D}=0.1$, yaw angle $=90^{\circ}$ from reference [58] (b) and numerical simulations (c) 173

Figure 5-47 Streamlines for cavity with $\mathrm{h} / \mathrm{D}=0.1$ at $90^{\circ}$ yaw angle at $\mathrm{z} / \mathrm{D}=0$ (a) and $\mathrm{z} / \mathrm{D}$ $=0.35(\mathrm{~b})$

Figure 5-48 Two-dimensional RANS computation of the flow pattern for $h / D=0.1$ [123]

Figure 5-49 Comparison of $\mathrm{C}_{\mathrm{p}}$ profiles on the centreline of the cavity base at yaw angle $=$ $90^{\circ}$ from numerical simulations (a) and reference [58] (b) 175

Figure 5-50 Comparison of drag coefficient at yaw angle $=90^{\circ}$ (The lines are added to visualize the general trend for each planform shape.) ............................. 176

Figure 5-51 Velocity deficit contours based on numerical simulations (left) and experimental measurements [58] (right) at $90^{\circ}$ yaw angle 178

Figure 5-52 Velocity deficit contours based on PIV measurements in a horizontal plane at $90^{\circ}$ yaw angle 179

Figure 5-53 Quantitative comparison of velocity deficit at $90^{\circ}$ yaw angle 179 
Figure 5-54 Streamwise vorticity contours at $\mathrm{x} / \mathrm{D}=1.0$ for $\mathrm{h} / \mathrm{D}=0.7$ (a) and $\mathrm{h} / \mathrm{D}=0.5$ (b) at $90^{\circ}$ yaw angle 180

Figure 5-55 Streamwise vorticity contours at $\mathrm{y} / \mathrm{D}=0.08$ for $\mathrm{h} / \mathrm{D}=0.7$ at $90^{\circ}$ yaw angle 181

Figure 5-56 $\mathrm{u}_{\mathrm{rms}}$ contours for $\mathrm{h} / \mathrm{D}=1.0$ (a), 0.7 (b), and 0.5 (c) at $90^{\circ}$ yaw angle at $\mathrm{z} / \mathrm{D}=$ 0. 182

Figure 5-57 Comparison of $\mathrm{u}_{\mathrm{rms}}$ (a) and $\mathrm{v}_{\mathrm{rms}}$ (b) profiles on the vertical centreline 182

Figure 5-58 The first four POD modes of the fluctuating streamwise velocity component 184

Figure 5-59 The first four POD modes of the fluctuating vertical velocity component. 184 Figure 5-60 The first forty normalized eigenvalues for $\mathrm{h} / \mathrm{D}=1.0$ (a) and 0.5 (b) at $90^{\circ}$ yaw angle at $\mathrm{z} / \mathrm{D}=0$ 185

Figure 5-61 Normalized time-varying coefficients for the cavities with $\mathrm{h} / \mathrm{D}=1.0$ (a) and $0.5(\mathrm{~b})$ 185

Figure 5-62 Phase averaged streamtraces in the vertical plane located at $\mathrm{z} / \mathrm{D}=0$, for the cavity with $\mathrm{h} / \mathrm{D}=1.0$. The phase interval between consecutive plots is $90^{\circ} 186$

Figure 5-63 Phase averaged streamtraces in the vertical plane located at $\mathrm{z} / \mathrm{D}=0$, for the cavity with $h / D=0.5$. The phase interval between consecutive plots is $90^{\circ} 186$

Figure 5-64 Schematic representation of the flow structure in an elliptical cavity in the nominally two dimensional-deep flow regime 188

Figure 5-65 Schematic representation of the flow structure in an elliptical cavity in the nominally two dimensional-intermediate depth flow regime 188

Figure 5-66 The location of shear layer centre at $30^{\circ}$ yaw angle 192

Figure 5-67 Shear layer thickness for $\mathrm{h} / \mathrm{D}=0.5$ at different yaw angles 193

Figure 5-68 Vortex core positions and $C_{p}$ contours at yaw angle $=15^{\circ}$ for $h / D=1.0,0.5$ and 0.1 195

Figure 5-69 Vortex core positions and $C_{p}$ contours at yaw angle $=30^{\circ}$ for $h / D=1.0,0.5$ and 0.1 196

Figure 5-70 Streamlines and $C_{p}$ contours for cavity with $h / D=1.0$ at $30^{\circ}$ yaw angle at $\mathrm{z} / \mathrm{D}=-0.7$ (a) and $\mathrm{z} / \mathrm{D}=0.7(\mathrm{~b})$ 197 
Figure 5-71 $\mathrm{C}_{\mathrm{p}}$ contours at yaw angle $=30^{\circ}$ for $\mathrm{h} / \mathrm{D}=0.5$ for a $2: 1$ elliptical cavity [58] and a 2:1 rectangular cavity (adapted from ref. [49]) 198

Figure 5-72 Vortex core positions and $C_{p}$ contours at yaw angle $=45^{\circ}$ for $h / D=1.0,0.5$ and 0.1 201

Figure 5-73 Vortex core positions and $C_{p}$ contours at yaw angle $=30^{\circ}$ for $h / D=1.0,0.5$ and 0.1 202

Figure 5-74 Instantaneous flow visualization image obtained using the Helium bubble technique, for the cavity with $\mathrm{h} / \mathrm{D}=1.0$ at yaw angle $=45^{\circ}$..... 203

Figure 5-75 $C_{p}$ contours at yaw angle $=45^{\circ}$ for $h / D=0.5$, Hering [58] (a), Savory and Toy (adapted from [5]) (b). 204

Figure 5-76 $C_{p}$ contours at yaw angle $=60^{\circ}$ for $h / D=0.5$ for a $2: 1$ rectangular cavity (adapted from ref. [49]).................................................................... 205

Figure $5-77 \mathrm{C}_{\mathrm{p}}$ distribution at two vertical planes inside the cavity ........................... 206

Figure 5-78 $\mathrm{C}_{\mathrm{p}}$ distribution contours on cavity base (a) and $\mathrm{C}_{\mathrm{p}}$ distribution contours and velocity vectors in three horizontal planes inside the cavity (b)-(d)........... 207

Figure 5-79 Schematic representation of the flow structure in an elliptical cavity in the strongly asymmetric flow regime 208

Figure 5-80 Comparison of drag coefficient for cavity with $\mathrm{h} / \mathrm{D}=0.5$ at various yaw angles (The lines are added to visualize the general trend for each planform shape.) 209

Figure 5-81 Velocity deficit contours based on numerical simulations (left) and experimental measurements [58] (right) at $45^{\circ}$ yaw angel 211

Figure 5-82 Streamwise vorticity contours at $\mathrm{x} / \mathrm{D}=0.7$ for $\mathrm{h} / \mathrm{D}=0.7$ at $45^{\circ}$ yaw angle211 Figure 5-83 Velocity deficit contours based on PIV measurements in a horizontal plane at $45^{\circ}$ yaw angle 212

Figure 5-84 Quantitative comparison of velocity deficit at $45^{\circ}$ yaw angle 213

Figure 5-85 Comparison of $\mathrm{u}_{\mathrm{rms}}$ (a) and $\mathrm{v}_{\mathrm{rms}}$ (b) profiles on the vertical centreline ....... 214 Figure 5-86 The first four POD modes of the fluctuating streamwise velocity component 215

Figure 5-87 The first four POD modes of the fluctuating vertical velocity component. 216 
Figure 5-88 The first forty normalized eigenvalues for $\mathrm{h} / \mathrm{D}=1.0$ (a) and 0.5 (b) at $45^{\circ}$

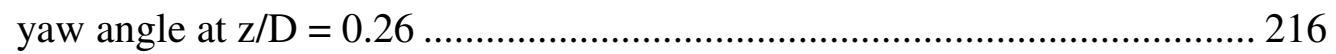

Figure 5-89 Normalized time-varying coefficients for the cavities with $\mathrm{h} / \mathrm{D}=1.0$ (a) and

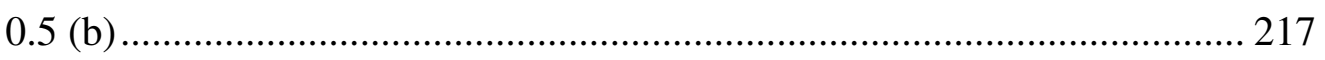

Figure 5-90 Phase averaged streamtraces in the vertical plane located at $\mathrm{z} / \mathrm{D}=0.26$, for the cavity with $\mathrm{h} / \mathrm{D}=1.0$. The phase interval between consecutive plots is $90^{\circ}$ 218

Figure 5-91 Phase averaged streamtraces in the vertical plane located at $\mathrm{z} / \mathrm{D}=0.26$, for the cavity with $\mathrm{h} / \mathrm{D}=0.5$. The phase interval between consecutive plots is $90^{\circ}$

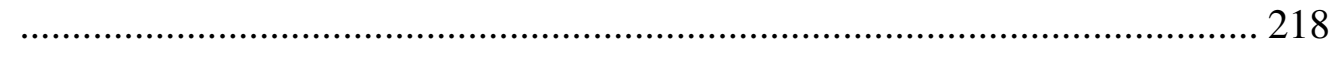

Figure 5-92 Comparison of $\mathrm{u}_{\mathrm{rms}}$ values for three different yaw angles at 0.1D downstream

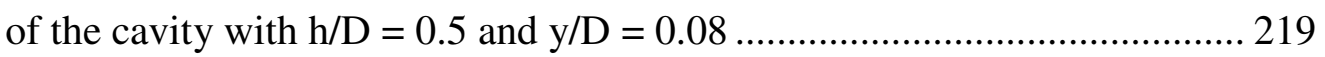

Figure 6-1 Modified classification of cavity flow regimes ....................................... 233 


\section{List of Appendices}

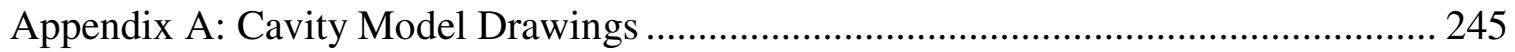




\section{Nomenclature}

\begin{tabular}{|c|c|}
\hline$A$ & Dimensionless drag parameter (function of $h / D$ ) \\
\hline$a$ & POD time varying coefficient \\
\hline$B$ & Dimensionless drag parameter (function of $M$ ) \\
\hline$C_{D}$ & Drag coefficient \\
\hline$\Delta C_{D}$ & Incremental drag coefficient due to cavity presence \\
\hline$C_{f}$ & Local skin friction coefficient \\
\hline$C_{p}$ & Mean pressure coefficient, $\left(\bar{P}-P_{\text {static }}\right) / P_{\text {dynamic }}$ \\
\hline$D$ & Cavity minor axis \\
\hline$e$ & Absolute uncertainty \\
\hline$h$ & Cavity depth \\
\hline$H$ & Boundary layer shape factor, $\delta^{*} / \theta$ \\
\hline$k$ & Turbulent kinetic energy \\
\hline$l$ & Turbulent length scale \\
\hline$M$ & Mach number \\
\hline$N$ & Number of samples \\
\hline $\bar{P}$ & Mean pressure \\
\hline$P_{\text {dynamic }}$ & Freestream dynamic pressure \\
\hline$P_{\text {static }}$ & Freestream static pressure \\
\hline$r m s$ & Root mean square, $\sqrt{\bar{u}^{2}}$ \\
\hline $\operatorname{Re}_{D}$ & Reynolds number based on cavity minor axis, $U_{\text {inf }} D / v$ \\
\hline$S_{i j}$ & Shear strain rate \\
\hline$S_{t}$ & Stokes number \\
\hline$U_{\text {inf }}$ & Freestream velocity \\
\hline$U_{\text {ref }}$ & Reference velocity \\
\hline $\bar{u}$ & Mean velocity \\
\hline$u_{\lambda}$ & Kolmogorov velocity scales \\
\hline$u^{\prime}$ & Rms value of fluctuating streamwise velocity \\
\hline
\end{tabular}




\begin{tabular}{|c|c|}
\hline$\overline{u^{\prime} u^{\prime}}$ & Reynolds stress component in streamwise direction \\
\hline$W$ & Cavity width \\
\hline$x$ & Streamwise coordinate \\
\hline$y$ & Vertical coordinate \\
\hline$z$ & Spanwise coordinate, probability of estimating the mean value within $\pm e$ \\
\hline$\alpha$ & Trailing edge flap angle \\
\hline$\Gamma$ & Circulation \\
\hline$\delta$ & Boundary layer thickness (based on $u=0.99 U_{i n f}$ ) \\
\hline$\delta^{*}$ & Boundary layer displacement thickness \\
\hline$\delta_{\omega}$ & Vorticity thickness \\
\hline$\varepsilon$ & Rate of strain, Dissipation rate \\
\hline$\theta$ & Boundary layer momentum thickness \\
\hline$\kappa_{r}$ & Real part of the wave number \\
\hline$\Lambda$ & Integral length scale \\
\hline$\lambda$ & POD eigenvalue, Kolmogorov length scales \\
\hline$\mu$ & Dynamic viscosity \\
\hline$\mu_{t}$ & Turbulence viscosity \\
\hline$v$ & Kinematic viscosity \\
\hline$\rho$ & Density of air \\
\hline$\sigma_{x}$ & Standard deviation \\
\hline$\tau^{\prime}$ & Shear stress across cavity mouth \\
\hline$\tau_{f}$ & Characteristic time scale of the fluid flow \\
\hline$\tau_{f D}$ & Time scale of macroscopic fluid motion \\
\hline$\tau_{f \Lambda}$ & Time scale of integral-scale turbulent motions \\
\hline$\tau_{f \lambda}$ & Time scale associated with Kolmogorov scale \\
\hline$\tau_{P}$ & Response time of a seeding particle \\
\hline$\tau_{w}$ & Wall hear stress \\
\hline$\varphi$ & POD Mode (eigen function) \\
\hline$\psi$ & Stream function \\
\hline$\Omega_{i j}$ & Vorticity magnitude \\
\hline$\omega$ & Vorticity \\
\hline
\end{tabular}




\section{Introduction}

The flow over cavities (surface cut-outs) is relevant to the aerodynamics of aircraft and road vehicles and has been investigated since the early 1930s. The main focus of the research has been drag reduction by removing or modifying cavities and reducing the noise associated with them. Drag reduction is a major challenge in aerodynamic design but there is usually room for improvement. For a typical civil transport aircraft, parasitic drag, which originates from skin friction, surface imperfections, and pressure drag of the non-lifting components, is about 3\% of the total drag [1]. Reduction of the parasitic drag can improve the vehicle's fuel economy without affecting its aerodynamic performance. As an example of the significance of aerodynamic refinement through drag reduction, a $1 \%$ reduction in aerodynamic drag for an Airbus A340 airplane operating in the long range mode will result in savings of about 400,000 litres of fuel per year [2].

Surface irregularities are mostly caused by design or manufacturing constraints on aircraft or road vehicles. These include landing gear wells, weapons bays, flap recesses, rivet depressions and recessed windows for airplanes [3] and truck beds, sun roofs and door gaps for automobiles [4]. Most of these examples can be adequately approximated by models of simple geometry such as cavities of rectangular, circular or elliptical planforms. This highlights the importance of a thorough understanding of the flow associated with three-dimensional cavities of various planform shapes and depths.

The present study is focused on three-dimensional cavities with elliptical planforms, as one of the most general and complex forms of cavities. Compared to three-dimensional cavities with rectangular planforms, the flow associated with an elliptical cavity is more complicated due to the curved sidewalls. Furthermore, unlike a three-dimensional cavity with a circular planform, the flow associated with an elliptical cavity is affected by yaw angle, due to its elongated planform shape.

A comprehensive review of the studies on cavity flows has been carried out and summarized in the next chapter. In summary, these studies indicate that the cavity flow regime varies with geometric parameters, including depth to length ratio, aspect ratio, 
planform shape, and yaw angle, as well as flow related parameters, including Reynolds number (laminar or turbulent flow), approaching boundary layer parameters, and Mach number. These parameters have been shown to have significant effects on all aspects of the flow inside and around cavities, including flow structure and vortices, pressure distribution and aerodynamic forces, and flow dynamics. It is therefore not adequate to generalize the findings related to cavities with a given set of geometric and flow parameters to other combinations of these parameters, which may require dedicated studies.

Although many researches have been performed on cavity flow over the past eighty years, most of them have been focused on nominally two-dimensional rectangular cavities subject to laminar flows. Only a few studies were focused on three-dimensional cavities, which were mostly confined to rectangular planforms, parallel to the flow.

The elliptical cavities have been studied by a few investigators. Friesing [3] studied the effect of elliptical cavity depth and aspect ratio on the resulting drag. Savory and Toy [5] have studied the effect of yaw angle and cavity depth for the elliptical cavities with aspect ratio of 2:1 and noticed an increase in drag for certain yaw angles. Hering and Savory [6] have studied the elliptical cavities with aspect ratio of 2:1 with different combinations of cavity depths and yaw angles. They have investigated the associated drag for each case, and presented a classification of different flow regimes for elliptical cavities. The limited number of studies on elliptical cavity flow has left many gaps in this research field. These gaps are discussed in detail in the next section.

\subsection{Gaps in Research on Elliptical Cavities}

The review presented in Chapter 2 indicates that the physics of the flow associated with elliptical cavities has not been completely determined, because of the limited number of previous studies in this area. Certain gaps exist in the knowledge on the flow associated with elliptical cavities, which can be summarized as follows:

- While the three-dimensional effects caused by the presence of sidewalls, such as variations of the flow parameters across the span, and formation of three- 
dimensional vortical structures, are relatively well-documented for cavities with straight walls (rectangular cavities), little is known about these effects in the case of cavities with curved walls.

- Elliptical cavities have been found to have highly asymmetric flows at certain yaw angles, and drag forces larger than those of comparable rectangular cavities. This behaviour has been observed in a few previous studies, based on surface pressure distributions and wake velocity measurements; however, the flow structure at these conditions has not been investigated in detail, and therefore little knowledge of the mechanisms leading to this behaviour is available

- The effect of yaw angle on flow structure, pressure distribution, and drag of elliptical cavities is different for cavities with different depths (deep, intermediatedepth, and shallow cavities). Specifically, the high drag associated with strongly asymmetric flows is observed only for deep and intermediate depth cavities. The reason for this behaviour has not been explained.

- Previous studies on elliptical cavities include surface pressure measurements, which can provide evidence of the flow structure near the cavity surfaces. No measurement of the flow inside an elliptical cavity, which would make it possible to establish a detailed and comprehensive three-dimensional representation of the flow structure inside the cavity, has been reported in the past. Also, the knowledge on dynamics of the flow inside elliptical cavities is limited to pointwise measurements using hotwire anemometry or surface pressure data, and no description of the dynamic behaviour of the principal flow structures (such as the shear layer and vortices) are available, in the case of elliptical cavities.

\subsection{Hypotheses}

The present study has been defined to address some of the above-mentioned gaps in the knowledge on the flow associated with three-dimensional cavities with elliptical planforms, to provide a better understanding of the flow structure, the parameters affecting the flow, and the underlying fluid mechanic mechanisms. 
Definition of the objectives, direction, and scope of the research is based on the following hypotheses, which will be evaluated throughout the thesis:

- Significant changes occur in the flow, when a nominally two-dimensional cavity is bounded by sidewalls. These changes are more pronounced for cavities with low aspect ratios. It is expected that the curvature of the sidewalls leads to additional three-dimensional effects in the flow, such as curvature and displacement of recirculation regions and the associated vortices, and more complicated secondary flow structures, and gives rise to flow features unique to elliptical cavities, especially for low aspect ratios.

- Different flow regimes have been observed in both nominally two-dimensional and finite aspect ratio rectangular cavities, depending on cavity depth. Similarly, the flow regime in elliptical cavities is also expected to be dependent on the cavity depth, but the flow structure in these regimes may not necessarily be similar to those of the aforementioned cavities, due to the presence of curved walls.

- It is hypothesized that various asymmetric flow structures exist in elliptical cavities, depending on the combination of yaw angle and cavity depth. These flow structures are expected to have unique features due to the elliptical planform shape. It is also conceivable that the high drag observed for elliptical cavities at certain yaw angles is related to asymmetric flow structures similar to that of the circular cavity with $\mathrm{h} / \mathrm{D}=0.5$.

- Considering previous studies on nominally two-dimensional and rectangular cavities, it is conceivable that certain aspects of the separated shear layer (such as shear layer centre position) change according to depth and yaw angle of an elliptical cavity, while other aspects (such as shear layer growth rate) remain unaffected. The quantitative values of flow parameters inside the cavity are also expected to be related to those of the separated shear layer, rather than the freestream. 
- Based on the relationship between position of the vortex core and the lowpressure regions inside the cavity, which will be verified for elliptical cavities in the present study, a quantitative relationship is expected to exist between vortex strength and pressure levels on cavity surfaces.

- The flow in the wake of the elliptical cavity is expected to show traces of the flow phenomena caused by presence of the cavity. Depending on these phenomena, which may include the separation bubble formed immediately downstream of the cavity, side-edge vortices, and trailing vortices formed in certain asymmetric flow regimes, the wake may be dominated by velocity deficit or vorticity. Drag of the cavity is expected to be larger in the cases in which the wake is dominated by velocity deficit.

- The dynamic phenomena of the flow in an elliptical cavity, including feedback resonance in the shear layer, shear layer oscillations, and vortex oscillations, are expected to be dependent on the combination of upstream boundary layer properties and state, geometric proportions of the cavity, and yaw angle. Therefore, it is conceivable for the flow in the cavity to show different levels of oscillation for different combinations of the above-mentioned parameters.

\subsection{Objectives of the Research}

In order to evaluate the above-mentioned hypotheses, the following objectives have been defined for the present research:

- To establish a comprehensive three-dimensional representation of the flow structure in the elliptical cavity volume, in order to complement previous studies in which the flow structure is determined based on surface pressure measurements.

- To determine the effects of wall curvature on the flow structure in elliptical cavities at various combinations of cavity depth and yaw angle, and through comparison with the flows associated with rectangular cavities. 
- To analyze the effect of depth on the flow structure inside and around elliptical cavities, in order to identify the flow regimes and features affected by depth and to determine similarities and differences with cavities with other planform shapes.

- To investigate the effect of yaw angle on the flow structure inside and around elliptical cavities with various depths, and to determine the flow structure and mechanisms associated with the increased drag forces associated with strongly asymmetric flows.

- To analyze the flow in the wake of the cavity, in order to investigate effect of the cavity flow phenomena on the wake

- To study the dynamic behaviour of the vortex structures in the cavity.

- To identify the underlying mechanisms from which the above-mentioned effects originate.

\subsection{Approach of the Current Investigation}

Fulfillment of the above-mentioned objectives requires investigation of the flow inside and around elliptical cavities for multiple combinations of geometric parameters, such as cavity aspect ratio, relative depth, and yaw angle, and flow parameters, including Reynolds number, and the thickness and state of the upstream boundary layer.

An investigation involving combinations of all of the above-mentioned parameters is beyond the time and facility resources available for the present research. Therefore, the scope of the present research has been defined by limiting these parameters to values that cover an adequate range of flow regimes, and make it possible to compare the results of the present study to previous ones involving elliptical and rectangular cavities.

With regards to the geometric parameters, an aspect ratio of $2: 1$ is chosen for the elliptical cavity. The reason for selecting this aspect ratio is that the present research aims at studying three-dimensional flows in cavities, which are associated with low aspect ratio cavities. This aspect ratio has been examined experimentally for three-dimensional 
cavities with rectangular planforms. The cavity with a circular planform (which is a special case of elliptical cavity with an aspect ratio of 1:1), has also been studied experimentally in the past, and interesting three-dimensional flow features have been reported in both cases. The elliptical planform with an aspect ratio of 2:1 has been chosen to examine the flow mechanisms in comparison with those reported with the abovementioned cases. Combinations of six ratios of cavity depth to minor axis length (h/D), including $0.1,0.2,0.35,0.5,0.7$, and 1.0 , and six yaw angles, including $0^{\circ}, 15^{\circ}, 30^{\circ}, 45^{\circ}$, $60^{\circ}$, and $90^{\circ}$ have been analyzed.

With regards to the flow parameters including Reynolds number, and the thickness and state of the upstream boundary layer, an attempt has been made to adjust them so that they match or are close to those of previous studies involving elliptical cavities. As will be shown in the coming chapters, this makes it possible to compare the observations and results of the present study with those of the previous work. Details of the flow related parameters will be described in Chapters 3 and 4 .

Particle Image Velocimetry (PIV) and CFD simulations are the most adequate measurement and analysis techniques for achieving the first objective, since they can be used to acquire information not only on the boundaries of the cavity, but also inside the cavity volume. Therefore, these techniques have been selected as the principal tools for the present study.

\subsection{Organization of the Thesis}

The methodologies implemented in the present research, the results of the measurements and simulations, and their analysis, are presented in the following chapters of this thesis, according to the following organization:

- In Chapter 2, a comprehensive review of the literature related to the flow in cavities, is presented. The review covers several topics, including fundamental concepts of vorticity, classification of cavity flows, effects of upstream conditions on cavity flows, cavity flow dynamics and oscillations, yawed cavities, different cavity planforms, drag of cavities, numerical simulations of cavity flow, PIV 
measurements of cavity flow. The findings of the reviewed research work have been summarized at the end of the chapter, to specify the framework within which the present research has been defined.

- Chapter 3 describes the details of the methodology of the numerical simulations conducted in the present study. The chapter includes detailed description of the governing equations and their discretization, description of the turbulence treatment approach, the simulation domain and its boundary conditions, the grid and sensitivity of the results to it, and validation of the numerical methodology adopted in the present study through comparison with previously published experimental data.

- Chapter 4 describes the details of the methodology of the experiments conducted in the present study. The chapter starts with a description of the experimental equipment, including the wind tunnel, the model, and the flow measurement and visualization equipment. This is followed by a description of techniques used to adjust the flow upstream of the cavity and the boundary layer parameters. The chapter also includes an analysis of the experimental uncertainties, considerations for selection of reference velocity, and methods for detection of vortex cores.

- Chapter 5 presents the results of the simulations and the experiments, with the objectives of providing a detailed explanation of the flow structure for various combinations of cavity depth and yaw angle, comparing the findings with those of previous studies involving elliptical cavities and other similar flows, and identifying underlying trends and flow mechanisms. The chapter starts with a qualitative and quantitative comparison of the results of the present study with previous studies involving elliptical cavities, to combine the results for description of the flow behaviour. This is followed by presentation and analysis of the results, including flow structure, pressure distribution, and flow dynamics, in three major categories elliptical cavity flows: Symmetric flow regimes, asymmetric flow regimes, and nominally two-dimensional flow regimes. The chapter ends with a 
summary, in which similarities and differences of the results of the present study with previous work are highlighted.

- Chapter 6 presents the conclusions of the present research. In this chapter, the findings presented in Chapter 5 are summarized with the objectives of identifying and describing the underlying trends and fluid mechanics phenomena leading to the observed flow structures, and highlighting the flow features unique to elliptical cavities. Based on the findings and trends observed in the present study, suggestions for future research are made.

\subsection{Summary}

This chapter has highlighted the need of fundamental research of flow over yawed, elliptical cavities with varying depths, where the flow physics are not well understood so far. The gaps in previous researches have been discussed and based on the observations in literature, some hypotheses have been presented. To evaluate the hypotheses, the research objectives have been defined and approaches to fulfill these objectives have been introduced. At the end the organization of the thesis and the major topics of each chapter were outlined. 


\section{Literature Review}

\subsection{Introduction}

In this chapter, a comprehensive review of literature is presented. Considering that the flow inside the cavity is dominated by vortices, the fundamental concepts of vorticity are discussed at the beginning of the review. This is followed by presentation of cavity flow classifications defined for nominally two-dimensional rectangular cavities.

Later, the effects of upstream flow condition, such as boundary layer characteristics and flow state (laminar or turbulent), on cavity flow are reviewed. The unsteady nature of cavity flows is discussed and flow dynamic mechanisms, including feedback resonance and conditions leading to it, are introduced. This is followed by a discussion on the principal factors affecting the cavity flow, such as yaw angle, depth and cavity planform shape. Cavity drag is another topic which is reviewed in this chapter.

It should be mentioned that most of the review of the cavity flows is based on experimental studies undertaken in wind or water tunnels. Computational fluid dynamics studies of cavity flows are discussed separately, to determine the best and the most feasible approach to perform the numerical simulation part of the present research.

Finally the key findings are summarized succinctly which leads to highlighting the gaps in the previous studies, which have been mentioned in section 1.1. The research hypotheses and objectives, as well as investigation approach have been defined accordingly in sections 1.2 to 1.4 .

In the following paragraphs, $\mathrm{D}$ refers to the stream-wise length of the cavity (chord), $\mathrm{W}$ is the span-wise width of the cavity and $h$ is the depth of the cavity. Aspect ratio is the cavity width to length ratio (W/D). Figure 2-1 shows these variables for a yawed rectangular cavity. 


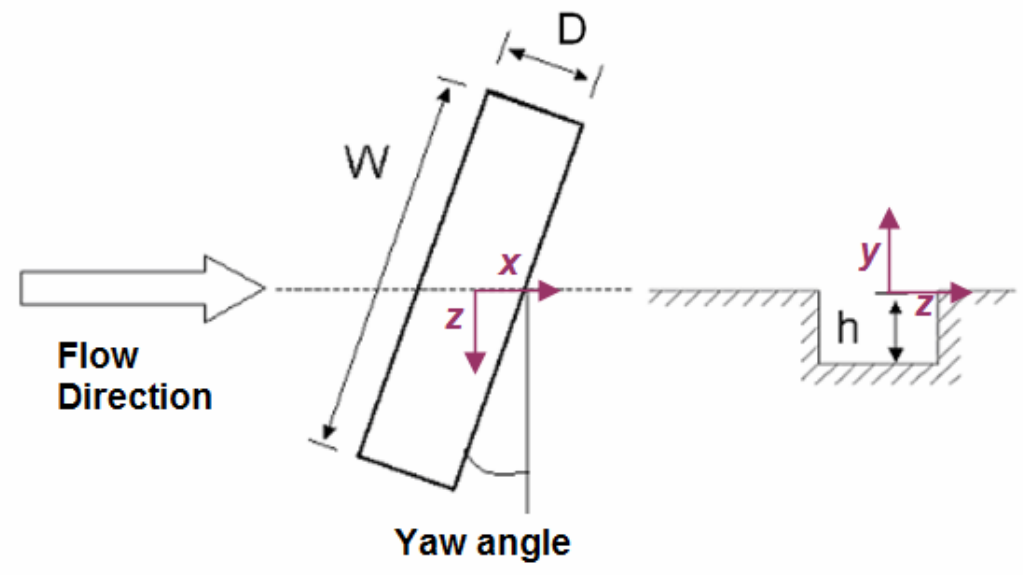

Figure 2-1 General cavity variables shown for a rectangular cavity

\subsection{A Brief Review of the Fundamental Concepts of Vorticity}

Flow in the cavity is dominated by one or more vortices, generated as a result of the interaction of the shear layer separated from the upstream edge of the cavity, and the cavity boundaries. Throughout the thesis, the vorticity contained in the shear layer, the vortices, and their interaction with the boundaries will be discussed in several instances. These discussions often rely on the fundamental concepts related to vorticity, which might not be mentioned in the discussion. The objective of this section is to provide a brief review of these concepts, which will support the discussions in the following chapters of the thesis.

The review includes some fundamental definitions, followed by an overview of the concepts of generation of vorticity, the relationship between vorticity and rate-of-strain, and diffusion and decay of vorticity.

The fundamental concepts of vorticity can be found in many texts in fluid mechanics. The present review is mostly based on the approach presented in references [7]-[11]. 


\section{- Definitions}

Conceptually, vorticity is defined as the local angular rate of rotation in a fluid. This conceptual definition leads to the mathematical definition of vorticity, as the curl of the velocity vector:

$$
\vec{\omega}=\vec{\nabla} \times \vec{u}
$$

A number of fundamental definitions are usually used to describe vorticity fields. As indicated by its definition, vorticity is a three-dimensional vector in a three-dimensional flow field. The line that is tangent to the vorticity vector in the flow field is called a "vortex line". It can be proved mathematically that vortex lines at a solid boundary are orthogonal to the streamlines [7].

Vortex lines can form closed loops (finite but endless paths) within the flow field, or extend to the boundary (solid, free-surface, or infinite), but cannot end within the flow field [8]. A "vortex tube" is a virtual closed surface formed by a series of vortex lines. It is usually assumed to have a circular cross section. The integral of the vorticity component normal to the cross section of a vortex tube, across the cross section is known

as circulation. Circulation, given by $\Gamma=\int_{S} \vec{\omega} \cdot \vec{n} d S$, is a measure of the strength of the vortex tube, and is constant along the tube in the absence of viscous effects, such as the "cross-diffusive" mechanism described later in this section, and turbulent decay of vorticity.

The equation governing transport and diffusion of vorticity in the flow field is known as the Helmholtz equation, or vorticity transport equation. This equation is written for an incompressible, homogeneous fluid as follows:

$$
\frac{D \vec{\omega}}{D t}=\frac{\partial \vec{\omega}}{\partial t}+(\vec{u} \cdot \vec{\nabla}) \vec{\omega}=(\vec{\omega} \cdot \vec{\nabla}) \vec{u}+v \nabla^{2} \vec{\omega}
$$

This equation plays a fundamental role in vortex dynamics, as it describes the vorticity field related to a discrete vortex from which vorticity is diffused into the flow field through viscous diffusion [7]. 
The material derivative on the right hand side of the equation shows the rate of change of vorticity (or angular velocity) for a fluid particle or element as it moves in the flow field. It accounts for the effect of unsteadiness in the flow (through the term $\frac{\partial \vec{\omega}}{\partial t}$ ), as well as movement of the fluid element from one point to another (through the term $(\vec{u} . \vec{\nabla}) \vec{\omega})$.

The first term on the left hand side of the equation $((\vec{\omega} \cdot \vec{\nabla}) \vec{u})$ is called the processing term. This term accounts for local amplification of vorticity because of velocity gradients. The effect of velocity gradients can be stretching of vortex filaments, or local tilting or turning of them. The second term on the left hand side of the equation $\left(\nu \nabla^{2} \vec{\omega}\right)$ accounts for diffusion of vorticity in the flow field due to viscosity [7].

\section{- Generation of vorticity}

The Helmholtz equation does not contain any generation term to account for creation of vorticity. The source of vorticity generation in homogeneous fluids is the boundary, and is included in the solution of the vorticity equation through wall boundary conditions. The physical effect of a wall boundary on the flow is the wall shear stress $\left(\tau_{w}=\left.\mu \frac{\partial u}{\partial y}\right|_{y=0}\right)$, where $\mathrm{y}$ is the wall-normal coordinate. The shear stress leads to a tangential force, which in turn causes angular acceleration, torque, and rotation of the fluid near the wall.

In the simplified case of a two-dimensional flow, the vorticity vector assumes the following form:

$$
\vec{\omega}=\{0, \partial u / \partial y-\partial v / \partial x, 0\}
$$

At the wall boundary, $\partial v / \partial x=0$, because the wall-normal velocity is zero. Therefore, the boundary conditions at the wall becomes:

$$
\omega_{0}=\{0, \partial u / \partial y, 0\}=\left\{0, \frac{1}{\mu} \tau_{w}, 0\right\}
$$


Therefore, $\omega_{0} \cdot \tau_{w}=0$. This is an important conclusion regarding the relationship between vorticity and wall shear stress. It implies that at the wall, vorticity is tangential to the surface, and at a $90^{\circ}$ angle to the shear stress. The other implication is that wall shear stress is not responsible for generation of vorticity. In fact, vorticity and wall shear stress are two representations of the wall-normal velocity gradient, related by $\mu$ [7] and [9]).

The time rate of change of circulation in the vicinity of the wall boundary can be considered as a measure of generation of vorticity. In a two dimensional flow field, circulation a thin closed circuit in the vicinity of the wall can be approximated by:

$$
\delta \Gamma=(u-U) \delta x
$$

in which $U$ is the flow velocity outside the near wall region. Circulation per unit length of the solid boundary is therefore $\Gamma=u-U$, and the time rate of change of circulation is:

$$
\frac{d \Gamma}{d t}=\frac{d u}{d t}-\frac{d U}{d t}
$$

Substituting the conservation of momentum equation $\left(\frac{d u}{d t}=\frac{\partial u}{\partial t}+u \frac{\partial u}{\partial x}=-\frac{1}{\rho} \frac{\partial p}{\partial x}+v \nabla^{2} u\right)$ in the above equation, leads to the following equation for generation of vorticity:

$$
\frac{d \Gamma}{d t}=-\frac{1}{\rho} \frac{\partial p}{\partial x}+v \nabla^{2} u-\frac{d U}{d t}
$$

Several conclusions regarding generation of vorticity can be made based on this equation. The formula indicates that vorticity generation results from acceleration or initiation of motion of a boundary in the tangential direction, and tangential pressure gradients along a boundary. In the case of a flat plate boundary layer in uniform flow, for example vorticity is generated at the leading edge of the plate (which in practice has a finite radius) at a rate of $\frac{1}{2} U^{2}$, and is then transferred downstream and diffused into the thickening boundary layer [9]. 
According to the equation above, a change in the direction of wall acceleration or pressure gradient leads to generation of vorticity with opposite sense at the wall. Also, viscosity plays no role in generation of vorticity, but it has a major role in redistribution of vorticity after generation. The formula also indicates that vorticity generation is instantaneous, and once it is generated, it cannot be lost by diffusion to the wall boundaries ([7] and [10]).

\section{- Vorticity and rate-of-strain}

Rate-of-strain in a two-dimensional flow is given by:

$$
\varepsilon=\partial u / \partial y+\partial v / \partial x
$$

There are extreme cases of a flow in which vorticity can exist without rate of strain, or vice versa. For example, in a flow with pure rigid-body type rotation, uniform vorticity exists, without any rate-of-strain. However, in most cases, a combination of both exists in the flow.

For example, in a shear flow with parallel streamlines, in which velocity is different on each streamline, a specific relationship exists between vorticity and rate-of-strain. The flow motion near the wall boundary is parallel to the wall. Therefore, the wall acts as a

source of both vorticity and rate-of-strain. At the wall, $\partial v / \partial x=0$, and rate of strain is equal to vorticity. This is the reason why there is vorticity but no large scale vortex structures in a laminar boundary layer.

The relationship between vorticity and rate-of-strain can be used to identify vortices in a region where both vorticity and rate-of-strain exists. This is the concept of the Q-criterion for identifying vortices, which subtracts rate-of-strain from vorticity, to identify the boundary of a vortex [11].

$$
Q=\frac{1}{2}\left(u_{i, i}{ }^{2}-u_{i, j} u_{j, i}\right)=\frac{1}{2}(\|\Omega\|-\|S\|)
$$


In the above-mentioned formula, $S$ and $\Omega$ are the symmetric and anti-symmetric components of $\nabla u$; i.e. $S_{i j}=\frac{1}{2}\left(u_{i, j}+u_{j, i}\right)$ and $\Omega_{i j}=\frac{1}{2}\left(u_{i, j}-u_{j, i}\right)$. Thus, Q represents the local balance between shear strain rate and vorticity magnitude. It should be noted that unlike vorticity, rate-of-strain can be generated not only at the boundaries, but also inside the fluid. Examples and more detail can be found in Reference [7].

\section{- Decay of vorticity}

The Helmholtz equation (Equation 2-2) and the equation of vorticity generation (Equation 2-7) show that vorticity cannot be lost by diffusion to the solid boundaries. The decay and loss of vorticity in a flow field occurs by a mechanism called "cross-diffusive" annihilation [7] and [9]). The concept of this mechanism is based on the fact that vorticity of opposite signs is generated at different regions (or time intervals) in the flow field. When the vorticity of opposite signs is transferred into the flow field, they interact and cancel each other. This leads to net loss of vorticity in the flow field.

Morton cites the global condition for circulation, which states that the gross circulation in an enveloping contour of a bounded region with outer boundary at rest must be zero, and concludes that at any given time, there exists an equal strength of vorticity of opposite signs within the domain, which may in time lead annihilation of vorticity when they diffuse and interact, depending on the flow structure. This principle can be verified for several types of flow, which are bounded completely or in part by solid boundaries [7].

\section{- Diffusion of vorticity}

Vorticity is a diffusive quantity and the mechanism by which it diffuses is viscous diffusion. Diffusion of vorticity is quantified by the $v \nabla^{2} \vec{\omega}$ term in the Helmholtz equation. Unlike linear momentum, diffusion of vorticity does not occur at the molecular level. Therefore, vorticity is categorized as a "continuum variable" which is transported in the flow field as a consequence of the diffusion of linear momentum (represented 
by $\nu \nabla^{2} \vec{v}$ in the Navier-Stokes equations), which in turn is related to velocity gradients in the flow field.

The cavity flow structure is dominated by vortices, and is strongly influenced by vorticity dynamics. Many of the cavity flow studies that will be reviewed in the following sections focus on global phenomena in the cavity, such as drag, pressure distribution, and acoustics. The present study, however, focuses on the flow structure in the cavity, including vortices and their dynamic behaviour. The principles described in the previous paragraphs provide a theoretical basis for the analysis and description of the behaviour of these vortices, which will be presented in Chapter 5.

\subsection{Cavity Flow Classifications}

In the case of depressions or cavities, simple geometries were initially investigated to examine the effects of cavity dimensional ratios on aerodynamic drag. Most of these studies were conducted on two-dimensional rectangular slots normal to the flow direction [4] and [12]. In later studies, fully three-dimensional cavities with different geometries and approaching flow conditions have been examined. These investigations have shown that the cavity flow regime varies with geometry (depth to length ratio, aspect ratio, and planform shape), yaw angle, Reynolds number (laminar or turbulent flow), approaching boundary layer parameters, and Mach number [13]-[58].

In 1961, the effects of depth to length ratio for two-dimensional rectangular cavities were studied and classic definitions of the different flow regimes were introduced [13]. Distinct flow patterns arise for certain conditions and, in general, cavity flows have been classified into two distinct categories, "open" and "closed" cavities with a transitional region between the two (Figure 2-2). These categories are based upon rectangular twodimensional cavities, where the spanwise width (W) is much larger than the length (D) and end effects can be neglected. Although the terms were first proposed for twodimensional rectangular cavities, they are also used to classify cavities with different planform shapes. It should be noted that these flow patterns relate to the time-averaged flow, whilst the flow unsteadiness will be discussed later. 
The term "closed" cavity refers to the case where the shear layer separates at the leading cavity lip and impinges on the cavity base near the middle of the cavity, as seen in Figure 2-2-(a). The shear layer then separates from the cavity base and passes over the downstream wall of the cavity. A stagnation point exists near the cavity lip of the downstream wall. In this case two regions of flow recirculation exist, one aft of the rearward-facing front wall, the other ahead of the forward-facing rear wall [14]. This flow regime occurs for shallow cavities where the cavity depth is much smaller than the cavity length $(\mathrm{h} / \mathrm{D}<<1)$.

The "Open" cavity regime occurs for cavities in which $h / D>0.1$ in the case of a rectangular cavity planform. In this regime, the shear layer separates at the upstream cavity lip and bridges the cavity opening. The stagnation point occurs slightly closer to the cavity lip on the downstream cavity wall when compared to the closed flow [14]. A stable vortex forms inside the cavity volume for open type flows, as noted by Roshko [4]. Different configurations are possible for the stable vortex depending on the ratio $h / D$. This captive stable vortex is driven by the separated shear layer spanning the cavity [4].

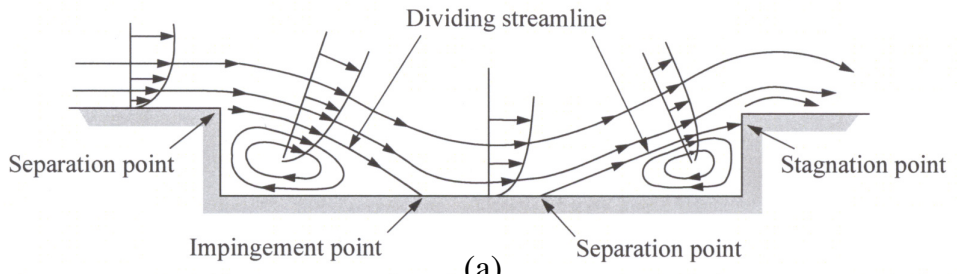

(a)

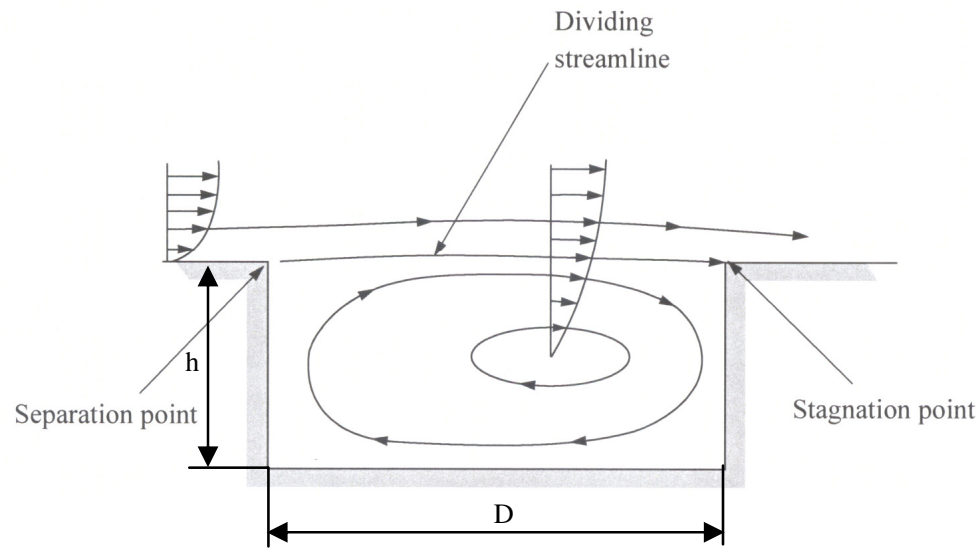

(b)

Figure 2-2 Closed (a) and open (b) cavity flow regimes at subsonic speeds (adapted from ref. [14]) 
The flow regimes are strongly affected by Mach number. The same categorization is possible for supersonic speeds as well. In case of closed cavities expansion fans are generated at the upstream and downstream edges. For the closed regime, two shock waves, known as the impingement and exit shock waves originate from the cavity base. In the case of open cavities two shock waves exist close to the two edges (Figure 2-3). For supersonic speeds two transitional stages, known as transitional-open and transitional-closed, were identified [14].
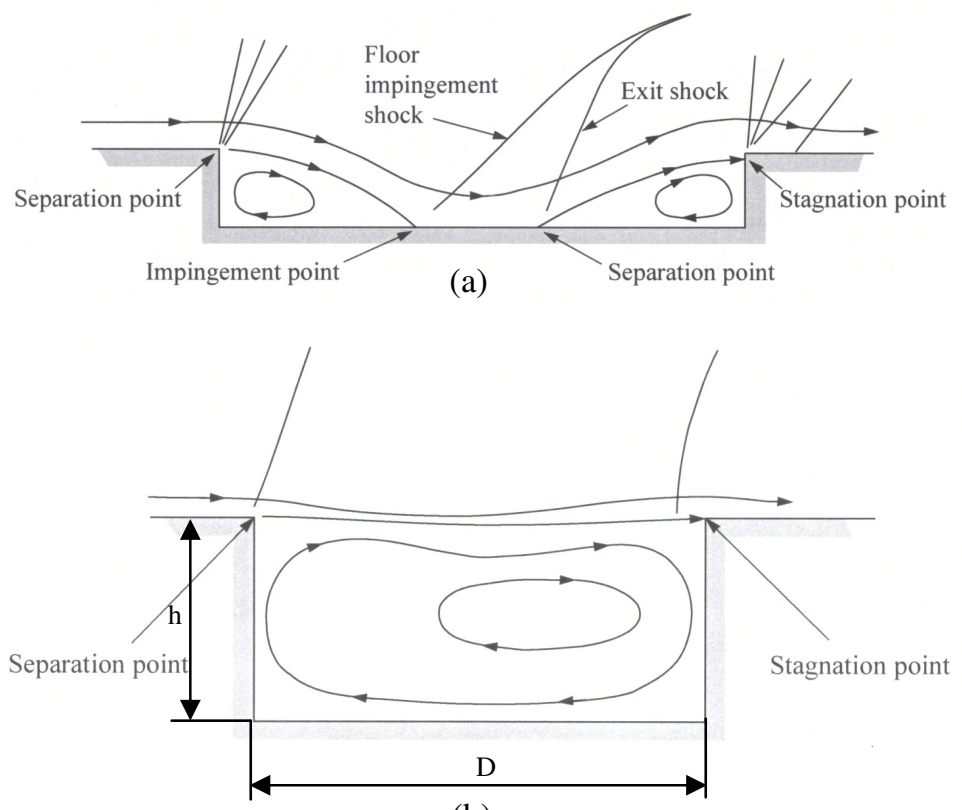

(b)

Figure 2-3 Closed (a) and open (b) cavity flow regimes at supersonic speeds (adapted from ref. [14])

Plentovich et al. studied the centre line ( $\mathrm{x}-\mathrm{y}$ plane at $\mathrm{z}=0$ on the cavity base) pressure coefficient pattern for a rectangular cavity subjected to subsonic $(0.2<\mathrm{M}<0.95)$ fully turbulent flow with a very thin boundary layer, to determine the effect of the flow regime on the centre line pressure distribution. They realized that for open flow the pressure is approximately uniform for a considerable extent and then increases near the downstream wall. For closed flow a plateau occurs and downstream of the plateau the pressure decreases to form a minimum, followed by a rise in pressure to a maximum. For transitional flow, the pressure over the forward portion is similar to that of the open flow, and over the rearward portion is concave down (Figure 2-4) [15]. 
The other important geometrical aspect of cavity flow is the width of the cavity. Early studies have shown that, even in cavities with large span-to-chord ratios which would normally be considered to have two-dimensional flow near their centre lines, the flow is in fact three-dimensional and wave like structures (also known as cellular structures) can be observed across the span of the cavity. The number of cellular structures was dependent on the aspect ratio and cavity depth. The study by Maull and East [16], which deals with rectangular cavities with varying aspect ratios of width to streamwise length (W/D) and depth to streamwise length (h/D) documents the cellular structures in detail for the open cavity flow regime beneath turbulent boundary layer. At a constant width to length ratio (W/D = 9), as the cavity depth was increased (h/D decreased from 2.5 to 0.5 ), cellular structures with a fewer number of cells were observed. Also, for a constant depth ( $\mathrm{h} / \mathrm{D}=0.5$ ), when the width to length ratio (W/D) was increased from 2 to 5.5 , the number of cellular structures increased from 2 to 6 . The case of W/D $=2$ and $h / D=0.5$, in which 2 cellular structures were observed, is of particular interest, as it resembles a part of the findings of the present study, which will be discussed in Chapter 5 .

Figure 2-4 Cavity base centreline pressure profiles (adapted from ref. [15]) 
For rectangular cavities where $\mathrm{W} / \mathrm{D}<1$ the side walls and corners greatly affect the flow inside the cavity. The schematic diagrams of flow structures for small width open and closed rectangular cavities at subsonic speeds (Figure 2-5) illustrate the possible threedimensional nature of the main flow [14].

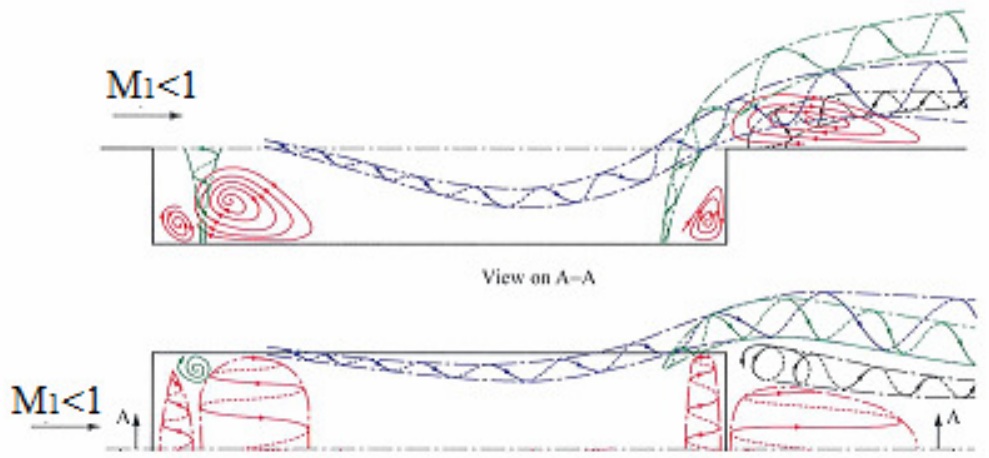

(a)
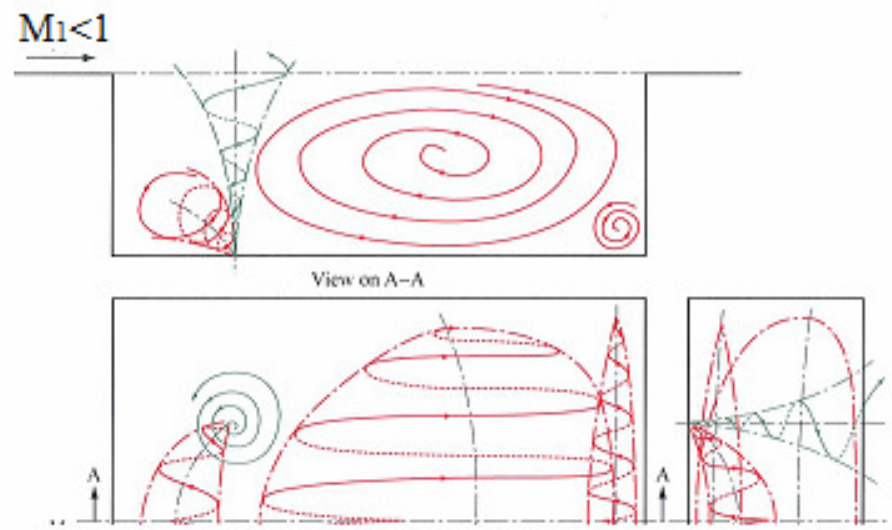

(b)

Figure 2-5 Main three-dimensional flow structures for rectangular closed (a) and open (b) cavities (adapted from ref. [14])

The flow patterns suggest the following main flow structures within the closed and open cavities:

\section{- Closed cavity (Figure 2-5-(a))}

There are two main regions of vortical flow aft of the front wall and forward of the rear wall as shown in Figure 2-2 earlier. The impingement line is curved due to the side wall 
effects. A very weak lateral vortex forms between the front wall and the forward separation line, making a counter rotating flow region. On the cavity base just aft of the front wall and close to the side walls, there are two weak separation nodes, which are associated with a pair of counter rotating vertical vortices. There are two other separation nodes on the cavity floor, close to the side walls and at the extremities of the separation line, which are indicated by a pair of counter rotating vertical vortices. While the shear layer cascades over the front wall, the flow is entrained over the side walls. Because of the recompression action of shear layer rising to pass over the rear wall, some of the entrained flow is ejected from the side walls.

When this schematic is compared to cavity base centreline pressure profiles (Figure 2-4), it can be observed that there is a low pressure area near the upstream wall of the cavity which is associated with the recirculation region at the upstream corner. Downstream of the recirculation region, the pressure rises again. This is then followed by a pressure drop, which can be attributed to the shear flow approaching the cavity floor. Then, another pressure rise is observed just before the downstream wall, due to a stagnation region formed when the entrained flow exits the cavity.

The results of previous studies on the flow inside and around rectangular cavities indicate that for the cases in which the length (streamwise dimension) of the rectangular planform is much larger than its width (spanwise dimension), streamwise vortices are formed along the edges of the cavity sidewalls [14]. The mechanism leading to formation of these vortices is the "cascading" of the shear layer over the upstream edge of the cavity, which leads to entrainment of the flow into the cavity over the sidewalls. When this entrained flow passes over the sharp, 90-degree edge of the cavity sidewall, "side edge vortices" are formed. Further downstream, these vortices are lifted clear of the downstream wall, and extend into the downstream flow. This is due to the effect of the recompressing shear layer, rising to pass over the downstream wall of the cavity. The results show that decreasing the width of the rectangular cavity compared to its depth leads to stronger entrainment of flow over the edges of the sidewalls and, therefore, stronger side edge vortices. The side edge vortices are shown in Figure 2-5-(a). 


\section{- Open cavity (Figure 2-5-(b))}

There are tandem rotating vortices, in the central spanwise region. There is also a small, weak corner vortex, which has a sense of rotation opposite to that of the strong rear vortex. Also there are two separation nodes on the cavity floor just aft of the front wall, which indicate a pair of counter rotating vertical vortices.

For deep open cavities, one large captive vortex usually dominates the flow and the two recirculation areas associated with corner vortices are either small or negligible. Therefore, as shown in Figure 2-4, the pressure on the centreline is more or less uniform, for the most part, and then increases near the downstream wall of the cavity. However, for open-transitional flow regimes, the upstream recirculation zone has a non-negligible size. In these cases, the pressure distribution shows a lower pressure near the upstream wall when compared to the open cavity flow type.

Hunt et al. (1978) have established a criterion for consistency of topological representations of flow structures [17]. This criterion can be used to verify whether or not a topological representation, which can be based on flow visualizations, measurements or simulations, is compatible with the principles of kinematics, which require a finite, continuous velocity field. The criterion can be summarized as follows:

$$
\sum\left(N+\frac{1}{2} N^{\prime}\right)-\sum\left(S+\frac{1}{2} S^{\prime}\right)=1-n
$$

where $\mathrm{N}$ is the number of node points, $\mathrm{N}^{\prime}$ is the number of half-nodes, $\mathrm{S}$ is the number of saddle points, and $\mathrm{S}^{\prime}$ is the number of half-saddle points. Nodes and saddle points represent critical point in the flow field, for which a detailed mathematical definition can be found in the work of Hunt et al. [17]. From the point of view of practical application of this criterion, a vortex core can be considered an example of a node, while separation, reattachment, and stagnation points can be considered as practical examples of saddle points. Half-nodes and half-saddle points are nodes and saddle points forming on the solid boundaries of the flow field. Also, $\mathrm{n}$ is the surface connectivity parameter, which is 0 for an unbounded flow (such as a free shear layer), 1 for a flow bounded by a solid wall (such as the cavity flow), and 2 for flow around a solid object (such as a flying vehicle). 
In order to verify the consistency of the topological representations of the flow in open and closed cavities, shown in Figure 2-5-(a) and (b), nodes and saddle points have been identified and shown in Figure 2-6-(a) and (b). For the closed cavity case, 4 nodes representing the vortices, and eight half-saddle points representing separation, reattachment, and stagnation points are found. In the case of open cavity flow three nodes, and six half-saddle points are observed. Considering the connectivity parameter of $\mathrm{n}=1$ for the cavity flow, both representations balance Equation 2-10. It can be concluded that these topological representations of flow in open and closed cavities are physically plausible, satisfying the criterion established by Hunt et al. [17].

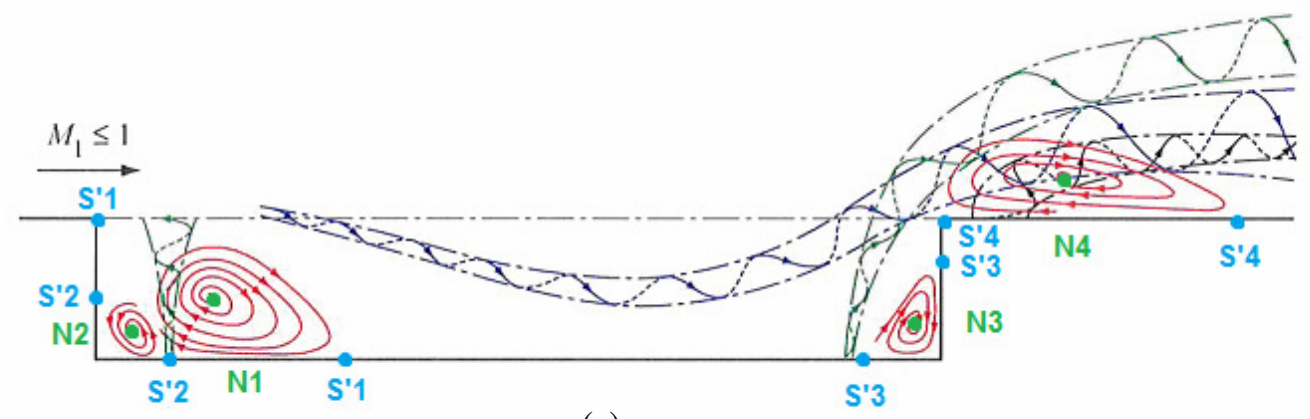

(a)

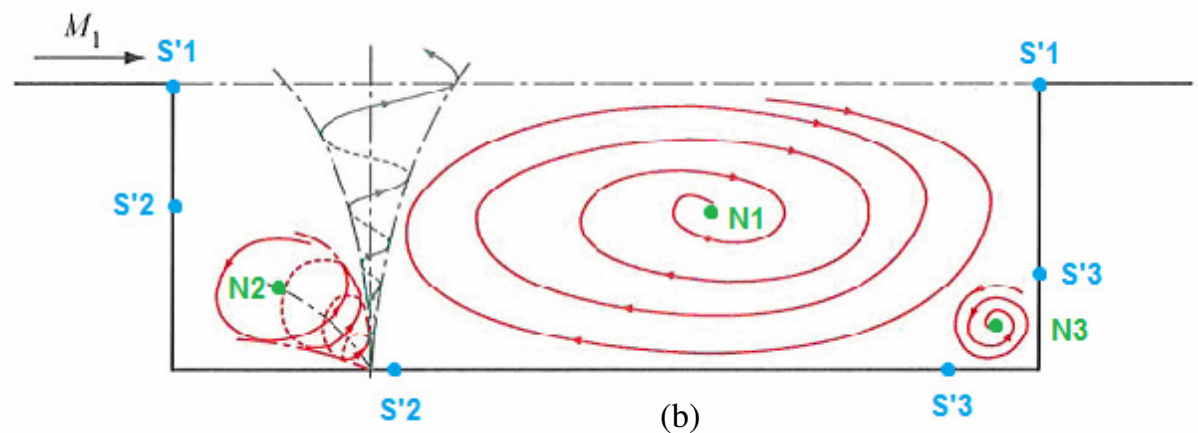

Figure 2-6 Topology of flow for closed (a) and open (b) rectangular cavities

\subsection{Effect of Upstream Conditions on Cavity Flow}

The condition of the boundary layer upstream of the cavity plays an important role on the characteristic of the flow inside the cavity. The thickness of the boundary layer relative to the depth of the cavity, and the state of the boundary layer (laminar or turbulent) are two 
important parameters in determining these effects. Considering fundamental similarities between flow in cavities and flow downstream of backward facing steps, the findings reported for backward facing steps will be used in this section to support the findings of the limited number of studies involving cavity flows.

\subsubsection{Cavity Flows}

Despite the importance of the effects of upstream boundary layer on the cavity flow, the studies in which these effects have been investigated systematically and described in detail are very few. However, there are a number of studies in which the results highlight the effect of boundary layer thickness on the flow inside the cavity implicitly.

Plentovich measured cavity wall pressure distributions in his study of rectangular cavities with depth to length ratios (h/D) between 0.09 and 0.23 at Reynolds numbers between $1.0 \times 10^{6}$ and $4.2 \times 10^{6}[18]$. His investigation included pressure measurements for two different upstream boundary layer thicknesses of $\delta / \mathrm{D}=0.021$ and 0.014 . The results of his study indicate that as the boundary layer thickness decreases, positive pressure levels inside the cavity increase. This effect has been attributed to higher velocities being entrained into the cavity. However, since only two relatively thin boundary layer thicknesses have been investigated, and no velocity measurements were taken in his study, these results can not be generalized.

In another example of the study of the effect of boundary layer thickness on cavity flow characteristics, Hering and Savory [6] compared measurements of pressure on the walls of an elliptical cavity exposed to a turbulent layer with $\delta / \mathrm{D}=1.08$, at $\operatorname{Re}_{D}=9.1 \times 10^{4}$ to the earlier results for a similar cavity exposed to a boundary layer with $\delta / \mathrm{D}=4.57$, at $\operatorname{Re}_{D}=5.6 \times 10^{4}$ [5]. Although the pattern of pressure distributions is similar in these two studies, the level of pressure coefficients is lower for the case with thicker boundary layer. Again, this behaviour has been attributed to lower velocities being entrained into the cavity. 
Atvars et al. [19] mention the effect of boundary layer thickness on the velocity at the edge of the cavity very briefly. Their study involves experimental and computational investigation of a transonic flow (with a Mach number of 0.85 , and $\operatorname{Re}_{D}=3 \times 10^{6}$ ) over a rectangular cavity with a depth to length ratio (h/D) of 0.2 . Even though they present results for one value of $\delta / D=0.12$, they mention that the ratio between the shear layer velocity and the freestream velocity is 0.57 for a "thin" boundary layer, and is expected to decrease as the boundary layer thickness increases. They do not specify if this ratio applies to the shear layer centre or any other location within the shear layer.

In recent years, non-intrusive and instantaneous measurement methods such as particle image velocimetry (PIV) have allowed detailed quantitative velocity data inside the cavities to be captured and have helped to gain a deeper insight into the physics of cavity flows [20]. Considering the limited and bounded space inside the cavities and the sensitivity of the flow due to any disturbance due to the measurement equipment, the importance of using non-intrusive techniques is highlighted.

With regards to the effect of the boundary layer state, a study by Haigermoser et al. [21] investigates the effect of the boundary layer state on growth of the separated shear layer downstream of the separation point, for nominally two-dimensional rectangular cavities with $\mathrm{h} / \mathrm{D}$ varying between 0.25 and 0.5 , and Reynolds numbers ranging between $1.0 \times 10^{4}$ and $3.2 \times 10^{4}$. Their results show that the thickness of the shear layer increases linearly in the streamwise direction along the edge of the cavity with a turbulent incoming boundary layer. The growth of the shear layer past the separation point can be evaluated by investigating the variations of vorticity thickness $\left(\delta_{\omega}\right)$. Vorticity thickness is defined as follows by Brown and Roshko [22]:

$$
\delta_{\omega}=\frac{U_{2}-U_{1}}{\left(\frac{\partial u}{\partial y}\right)_{M A X}}
$$

where $U_{2}=U_{\text {inf }}$ and $U_{1}=0$ in calculating vorticity thickness along the shear layer at the upper edge of cavities. 
The shear layer growth for cavities exposed to laminar boundary layers is found to be non-linear, with increasing growth rate for cavities with smaller h/D [21]. It was shown that the highest growth rate belongs to the cavity exposed to a turbulent boundary layer, indicating that more momentum is entrained into the cavity compared to a cavity of the same h/D ratio, exposed to a laminar boundary layer. The linear growth of the shear layer thickness in the case of turbulent boundary layer was also confirmed by other studies [23]-[26]. For nominally two-dimensional rectangular cavities, values between 0.114 and 0.3 have been reported for $d \delta_{\omega} / d x$ [21]-[26].

In the case of laminar upstream flow, three PIV measurements were performed on open nominally two-dimensional rectangular cavity flow. These studies were carried out on cavities with $\mathrm{h} / \mathrm{D}=0.25$ at $\mathrm{Re}_{D}=1.6 \times 10^{4}-5.2 \times 10^{4}$ [27], $\mathrm{h} / \mathrm{D}=0.2$ at $\operatorname{Re}_{D}=5 \times 10^{3}$ $2.9 \times 10^{4}$ [28], and $\mathrm{h} / \mathrm{D}=0.5$ at $\operatorname{Re}_{D}=7.7 \times 10^{2}-1.4 \times 10^{3}$ [29]. As expected, open type flow with two large counter rotating recirculation bubbles was observed. By increasing the Reynolds number, the upstream recirculation bubble became smaller and the downstream recirculation bubble centre moved toward the upstream edge and increased in size for all cases [27]-[29]. This behaviour was attributed to the increased energizing effect of the shear layer [27]. The location of the vortices in the cavity was studied statistically and it was concluded that the vortices with a sense of rotation similar to the main recirculation zone originated from the shear layer, while vortices of opposite sign originated in the flow near the downstream wall. The circulation of the vortices was found to be larger for vortices with larger cores [27]. Also, velocity fluctuations and Reynolds stress components were found to increase with increasing Reynolds number, and towards the downstream wall [28] and [29].

In the case of turbulent approaching flow, nominally two-dimensional rectangular cavities with $\mathrm{h} / \mathrm{D}=0.25-0.5$ at $\operatorname{Re}_{D}=1.0 \times 10^{5}-1.4 \times 10^{5}$ [23], $\mathrm{h} / \mathrm{D}=0.25$ at $\operatorname{Re}_{D}=1.9 \times 10^{4}$ [24], and $\mathrm{h} / \mathrm{D}=0.17$ and $\operatorname{Re}_{D}=2 \times 10^{5}-7.6 \times 10^{5}$ [25] were studied. It was observed that the flow inside the cavity was dominated by a large recirculation zone, accompanied by a smaller one close to the upstream wall. As the freestream Reynolds number increases, the centre of rotation moves from near the downstream wall to the cavity centre, which is similar to what happens in the case of a laminar approaching boundary layer [25]. Figure 
2-7 shows the mean velocity vectors and the streamlines of the mean velocity field. The streamlines illustrate not only the impingement of the mean shear layer onto the downstream wall, but also a second recirculation zone toward the upstream wall. The time-resolved results were used to study vortical structures with different scales in the shear layer, and it was observed that the vortical structures originating from the shear layer grew in size and formed large-scale coherent structures with increasing distance downstream of the cavity [23] and [24]. Haigermoser et al. calculated the mean pressure field based on velocity data, and found a low pressure region associated with the main recirculation region, and a smaller high pressure region on the downstream wall, associated with shear layer impingements [24].

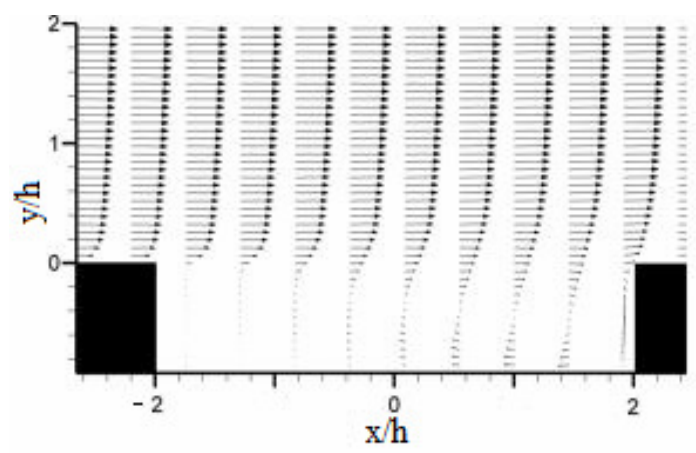

(a) Velocity field

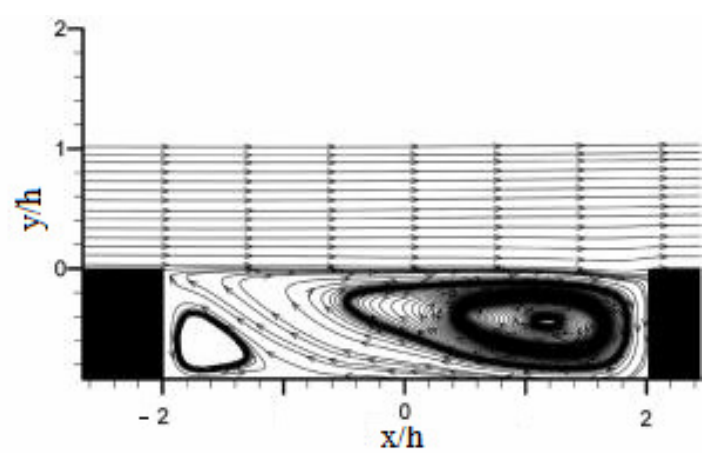

(b) streamlines

Figure 2-7 Mean flow field (adapted from ref. [24])

Also a periodic, low frequency oscillation of the shear layer was reported and attributed it to a flow instability leading to regular in- and outflow events [24]. Murray et al.'s study is one of the very few examples of application of proper orthogonal decomposition (POD) to cavity flows [25]. Their results show that although the relative energy of the dominant modes decreased at higher Reynolds numbers, the POD mode shapes at all Reynolds numbers were similar, indicating that the underlying dynamic structure of the flow is similar, despite different mean recirculation flow patterns. Their study also included some results regarding cavity resonance, a phenomena which is explained in section 2.5 . 
In another study, a wider range of $h / D$ (0.2-0.7) for nominally two-dimensional rectangular cavities with a turbulent boundary layer $\left(\operatorname{Re}_{D}=2.7 \times 10^{5}-3.3 \times 10^{5}\right)$ were investigated [26]. Their findings in the case of the shallow cavity $(\mathrm{h} / \mathrm{D}=0.2)$ were similar to those described in the previous paragraphs. In the case of the intermediate depth cavity $(\mathrm{h} / \mathrm{D}=0.7)$, Ukeiley and Murray reported one dominant circulation zone, driven by the shear layer [26]. Using quadratic stochastic estimation, they reconstructed the timedependent behaviour of the flow, and reported flapping of the shear layer regardless of resonant conditions, which they named "breathing". Unlike Ashcroft and Zhang [23], they found this phenomena to be non-periodic.

The above-mentioned research shows the capabilities Particle Image Velocimetry (PIV) as a non-intrusive technique for measurement of the complex flow in the volume of the cavity. Thanks to its capability of simultaneous velocity field measurement in multiple points in the flow field, PIV can provide valuable information about the flow structure that cannot be obtained otherwise. The effect of Reynolds number and h/D ratio on the position of the circulation zones inside the cavity, the spatial distribution and growth of velocity fluctuations and turbulent stresses downstream of the leading edge of the cavity, shear layer position and growth, and dynamic behaviour distinguished using POD, are examples of the valuable insight provided by PIV measurements of the cavity flow. When PIV measurements with sufficiently high frequency resolution are used, these data are completed with direct measurements of the time-dependent parameters of flow.

Although the above mentioned observations provide some insight into the effect of the upstream boundary layer on the cavity flow, a more in-depth understanding can be obtained by investigating these effects for other flow types which have fundamental similarities to cavity flows.

\subsubsection{Backward Facing Step Flows}

The flow over a backward facing step is one of the flow types which has fundamental similarities to the cavity, since it involves separation of a shear layer from a sharp edge, 
impingement of the shear layer onto a surface, and formation of a recirculation zone. The general structure of the flow is especially similar to that of a closed cavity.

Unlike cavity flows, several investigations have been carried out to study the effect of boundary layer thickness on the characteristics of the backward facing step flow. One of the earliest examples in which the effects of upstream boundary layer thickness and freestream turbulence on the flow behind a backward facing step have been investigated is the experimental study by Cheun et al. [30]. The study involves a backward facing step exposed to a turbulent boundary layer at a Reynolds number based on the step height of $\operatorname{Re}_{\mathrm{h}}=5.2 \times 10^{4}$. Combinations of two boundary layer thicknesses $(\delta / \mathrm{h}=0.14$ and $\delta / \mathrm{h}=$ $0.67)$ and two levels of freestream turbulence intensity $(0.5 \%$ and $3.5 \%)$ were investigated. The results indicated that for the case with thicker boundary layer the velocity profiles were broader, and had smaller gradients in the vertical direction, for all downstream locations (down to the reattachment region). The smaller gradients led to a larger recirculation zone, and reattachment was found to occur further downstream. The shear layer for the case exposed to a thicker boundary layer was thicker, and showed a larger growth rate downstream of the cavity. Smaller peaks of turbulence intensity and Reynolds shear stress were also observed for the case with thicker boundary layer.

With regards to the effects of freestream turbulence, the higher freestream turbulence has led to higher levels of turbulence intensity downstream of the step. The increase in turbulence intensity, indicated by the difference between turbulence intensity profiles at several downstream locations, is more pronounced for the case exposed to a thicker boundary layer. In general, the effects of increasing turbulence intensity have been reported to be less significant than the effects of increasing the thickness of the boundary layer.

The effects of boundary layer thickness on the flow characteristics of a nominally twodimensional backward facing step was investigated systematically by Adams and Johnston ([31] and [32]), for Reynolds numbers ranging from 800 to 40000, based on the step height. Their study involved variation of boundary layer thickness in the range of $0 \leq \delta / h \leq 2$. Their results indicated that for a turbulent incoming boundary layer $\left(\operatorname{Re}_{\mathrm{h}}=\right.$ 
$\left.3.6 \times 10^{4}\right)$, the peak pressure, pressure at the reattachment point, and the pressure downstream of the reattachment point decrease, when the boundary layer thickness increases. A thicker boundary layer also leads to a larger reattachment length due to a larger recirculation zone, in which the vortex core is located farther downstream from the step. The reattachment length is found to be generally larger for turbulent boundary layers.

Turbulence intensity and the overall level of shear stress in the recirculation zone also decrease when the boundary layer thickness increases. An increase in the thickness of the boundary layer has also been found to decrease the skin friction coefficient on the floor of the step. These observations are consistent with the previously described observations in the case of a cavity, and are indicative of lower velocities being entrained into the recirculation zones.

Some investigators have used axisymmetric backward facing step models to minimize the end effects, and achieve flows that are more similar to that of an infinite two-dimensional backward facing step. An axisymmetric backward facing step model comprises a circular cylinder with its axis aligned with the free stream, in which the step if formed by a sudden change of radius of the cylinder. The change in radius is usually chosen to be a small fraction of the radius of the cylinder, to minimize the effects of curvature at the step. Studies involving these types of models verify the trends originally established by Adams and Johnston ([31] and [32]) regarding the effects of boundary layer thickness.

Hudy [33] has studied the effect of boundary layer thickness on the flow characteristics of an axisymmetric backward facing step, by changing $R_{\mathrm{h}}$ between $6 \times 10^{3}$ and $1.9 \times 10^{5}$. In her study, the boundary layer thickness decreases as Reynolds number increases, therefore, the trend of variations if the flow characteristics with Reynolds number have been attributed to variations in the boundary layer thickness. The results indicate that friction velocity and skin friction coefficient on the floor of the step both decrease as the boundary layer thickens. Comparison of Reynolds shear stress profiles downstream of the step shows a larger peak (closer to the floor of the step) for higher Reynolds numbers 
(thinner boundary layers). The reattachment length is also found to be larger at lower Reynolds numbers, which correspond with thicker boundary layers.

In another similar study, Terekhov and Bogatko have investigated the effect of boundary layer thickness before separation on aerodynamic characteristics of an axisymmetric backward facing step [34]. The geometry in the study by Terekhov and Bogatko is different from that of Hudy, as it models the axisymmetric step as a sudden expansion in a cylindrical pipe. However, the general observations with regards to the effect of boundary layer thickness are similar. The simulations cover Reynolds numbers ranging from $\mathrm{Re}_{\mathrm{h}}=5 \times 10^{3}$ to $10^{5}$, and the effects of boundary layer thickness have been investigated by varying the boundary layer thickness between $\delta / \mathrm{h}=0.25$ and $\delta / \mathrm{h}=3$ at the constant Reynolds number of $\mathrm{Re}_{\mathrm{h}}=6700$. According to their results, as the boundary layer thickness increases, the peak pressure levels, and the skin friction coefficient in the recirculation zone decrease. Similar to the results of the study by Adams and Johnston ([31] and [32]), the reattachment length increases when the boundary layer thickens.

Another example, which involves a different problem and setting, but supports the abovementioned trend, is the study of flow in an urban-type street canyon by Savory et al. [35]. Their study involves two blocks with square profiles preceded by a series of cubic roughness elements of the same height, to simulate the interaction of the atmospheric boundary layer with two rows of buildings in an urban area. Velocity and turbulence intensity profiles have been compared near the upstream end of the cavity formed between the two blocks, for a cavity depth to length ratio of $h / D=0.1$. Their comparison with previous work involves two cases with Reynolds numbers close to one another, but with different boundary layer thicknesses at the separation point, due to different setup of roughness elements upstream. The comparison indicates that the case with thinner boundary layer shows a higher position of shear layer, and higher levels of turbulence intensity. Most importantly, the gradient of the streamwise velocity in the vertical direction, at the edge of the cavity, is much higher. These differences have been attributed by the authors to effects of several parameters, including boundary layer thickness. 
In previous paragraphs, a variety of studies involving various flow types and geometries have been reviewed to identify the effects of boundary thickness and state on the characteristic of the flow field formed by the separated shear layer. Although the flow types and the conditions of the experiments or simulations are different in the mentioned studies, certain general trends can be observed in all of them, which can be summarized as follows:

- A thicker upstream boundary layer results in lower velocities being entrained into the cavity or the recirculation zone. As a result the positive pressures in the cavity or recirculation region will be lower

- The parameters associated with the velocity gradients, such as skin friction coefficient and shear stress, will also be lower for a thicker upstream boundary layer

- The growth rate of the separated shear layer is larger for a turbulent boundary layer, compared to a laminar one, therefore, more momentum is entrained into the cavity exposed to a turbulent boundary layer.

For backward facing steps, the length of the recirculation zone, and consequently the distance between the vortex core and the upstream wall is larger for a thicker upstream boundary layer, and when the boundary layer is turbulent. Although the effect of the boundary layer thickness on the distance between the vortex core and the upstream wall has not been explicitly examined for cavities, there are a number of studies, in which the effect of free stream velocity on the recirculating flow inside the cavity are investigated.

\subsection{Cavity Flow Oscillations}

In reality, an open cavity flow is strongly unsteady because of the intense oscillations that occur in the cavity. These oscillations are self-sustained and classified in three main categories:

- fluid elastic oscillations due to coupling of the oscillations with the solid boundary motion, 
- fluid-resonant oscillations which can occur if the ratio of the cavity length (D) to the acoustic wave length is very small,

- fluid-dynamic oscillations due to velocity fluctuations in the turbulent boundary layer, shear layer instabilities, and fluctuations of the captive vortex within the cavity [36] and [37].

According to Rockwell and Naudascher [36], the physically significant parameters for fluid dynamic oscillations are: D/W, $\delta^{*} / \mathrm{D}, \theta / \mathrm{D}$, Reynolds number, and Mach number. One of the important phenomena resulting from fluid dynamic oscillations is feedback resonance. This is a special type of fluid-dynamic oscillation where the free shear layer is the main driving source of the oscillations. Feedback resonance refers to an interaction between a pressure pulse created at the downstream cavity lip by shear layer vortex stagnation and an unstable shear layer at the upstream cavity lip. Resonance occurs when a feedback loop develops along the cavity length, and amplifies distinct frequencies inside the cavity due to the instabilities in the shear layer. These resonant oscillations can cause structural fatigue and have been known to increase the drag by as much as $250 \%$ when compared to a non-resonating cavity [39].

As will be discussed in the following paragraphs, most oscillations of this type are only present for very thin approaching boundary layers and are suppressed by the level of turbulence in a thick turbulent boundary layer. Since, in the current study, the approaching turbulent boundary layer is thick $(\delta / \mathrm{D}=0.6)$, this type of oscillations does not occur. The main aspect of this type of oscillations will be briefly described in this section, for completeness of the review.

Rossiter described the feedback loop formation as follows (Figure 2-8) [38]:

1. Concentrated vortices are shed periodically from the upstream lip of cavity.

2. These vortices travel downstream along the shear layer which impinges the downstream wall of the cavity.

3. Upon interacting with the downstream wall of the cavity acoustic pulses are produced. 
4. These acoustic disturbances move upstream inside the cavity and disturb the shear layer at the upstream lip.

5. When the acoustic pulses reach the upstream end of the cavity, they cause the shear layer to separate upstream of the edge and shedding a new vortices.
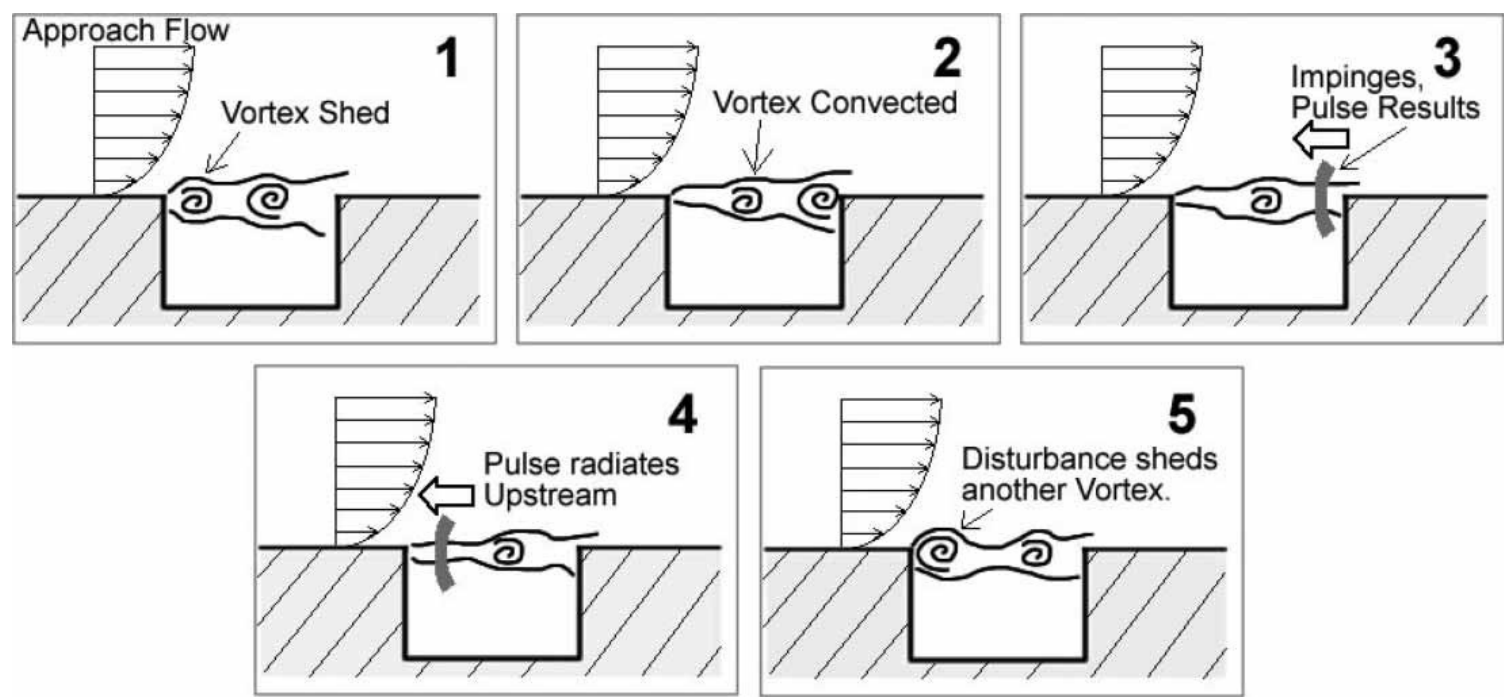

Figure 2-8 Typical feedback resonance mechanism [53]

Although Rossiter's semi-empirical formula was successful in some cases, it wasn't always predicting the tone frequency well, especially when the flow Mach number was outside the range covered in his experiments $(0.4 \leq M \leq 1.2)$ [41]. Later, Block modified Rossiter's formula by assuming that the acoustic waves reflected from the cavity base were also a key component [40]. Therefore, he suggested the following formula instead which has considerable effect on predicted frequencies for $M<0.4$ :

$$
f=\frac{U_{o}}{D} \frac{m}{M\left(1+\frac{0.514}{D / h}\right)+1 / \kappa_{r}}
$$

where $\kappa_{r}$ is the real part of the wave number of the disturbance traveling downstream. Both of these formulas predict the same frequencies for high Mach numbers. 
However, it should be noted that an open cavity flow does not always resonate. If $\delta$ is the boundary layer thickness immediately upstream of the cavity the, the following parameter ranges are necessary for cavity resonance:

1. Laminar upstream boundary layer

- Sarohia: $\delta / \mathrm{D}>0.075$ and $(\mathrm{D} / \delta)(\operatorname{Re} \delta) 0.5>290($ for $\delta / \mathrm{h}<0.5)[42]$

- Gharib and Roshko: 0.049<8/D<0.094 [43]

2. Turbulent

- Ahuja and Mendoza $\delta / \mathrm{D}<0.066$ [44]

Gharib and Roshko's experiments showed that for both $\delta / \mathrm{D}=0.06$ in one case and $(\mathrm{D} / \delta)\left(\operatorname{Re}_{\delta}\right)^{0.5}=285$ in another case, cavity is resonated which was in contrast with Sarohia's conditions. So, they suggested a new resonance condition [43]. They observed that when $\delta / \mathrm{D}>0.094$, the shear layer bridged smoothly over the cavity with no distinct oscillations. When $0.049<\delta / \mathrm{D}<0.094$, a self-sustained oscillation appeared in the cavity shear layer. The oscillations disappeared when $\delta / D<0.049$. In this case, the shear layer became unstable on a large scale comparable to the model dimensions, and no longer reattached at the downstream wall. This mode was called the wake mode [43].

Grace et al. have studied the effects of laminar and turbulent upstream boundary layers on a non-resonating cavity subject to low Mach number flow, using a removable turbulent trip at the entrance of the test section at the same velocity as laminar flow [45]. For the laminar flow, although $\delta / D=0.057$, the flow was non-resonating. This agrees with Sahoria's condition but is in contrast with Gharib and Roshko's findings. Also when they checked Sarohia's conditions for their experimental results, they realized that of the two parameters suggested by him, the dependence upon $\delta / \mathrm{D}$ is the strongest.

For the turbulent flow with $\delta / \mathrm{D}=0.127$ the cavity was non-resonating and it satisfies Ahuja and Mendoza's condition. However, Lin and Rockwell found cavity resonance for the approximately similar $\delta / \mathrm{D}$ of 0.113 in water [37]. The Reynolds number for both of the experiments was the same $\left(\operatorname{Re}=1.2 \times 10^{4}\right)$. The most significant parameter difference between the Lin and Rockwell's experiment and Grace's was the Mach number which 
due to the different fluid media, differed by a factor of 250 . Grace et al. concluded that the previously reported parameter ranges for resonance only remained valid at the specific Mach number and the upstream boundary layer condition for each experiment. Sarohia worked at $\mathrm{M}=0.02$ [42], Gharib and Roshko worked at $\mathrm{M}=0.00015$ [43] and Ahuja and Mendoza used M>0.25 [44].

In the case of circular cavities, since the streamwise length (D), which is used in Rossiter's formula, is not constant, his formula is not suitable for resonance frequency prediction. Marsden et al. have studied circular cavities with $\mathrm{h} / \mathrm{D}=0.5,1.0$, and 1.5 at Mach numbers $=0.15$ and 0.34 . They showed that the diameter-based Rossiter curves do not represent the Strouhal number variation with Mach number [46]. They concluded that the resonance phenomenon for circular cavities is more complicated than rectangular cavities.

\subsection{Yawed Cavities}

Yaw angle is another important parameter affecting the cavity flow regime. Wieghardt investigated the effects of yaw angle variation on drag coefficient of cavities with $\mathrm{W} / \mathrm{D}>8.15$ and $\mathrm{h} / \mathrm{D}=0.5$. Little variation of the drag coefficient was observed up to yaw angle $=35^{\circ}$. While drag increased up to $300 \%$ at yaw angle around $70^{\circ}$ to $80^{\circ}$. Also, he realized that shallower cavities $(\mathrm{h} / \mathrm{D}<0.25)$ were less affected by the yaw angle variation [47]. Savory et al. studied combinations of cavity depths and yaw angles for a W/D = 2 rectangular cavity. For yawed cavities, not only is the flow inside the cavity asymmetric, but vortices are also strongly shed, unlike the cases which the cavity is normal to the flow direction. Also, for some cases, namely $\mathrm{h} / \mathrm{D}=0.4-0.5$ and yaw angle $=45^{\circ}-60^{\circ}$, a sudden rise in the drag force is observed [48].

Czech studied different combinations of rectangular cavity W/D ratios and yaw angles experimentally and numerically and measured mean and unsteady pressures [49]. He showed that the cavity flow field changes considerably with aspect ratio, and that flow field inside the cavities of small aspect ratio (W/D $=2$ ) are less affected by changing the yaw angle. Also, for $\mathrm{W} / \mathrm{D}=4.85$ considerable fluid entrainment occurs near the upstream 
end of the downstream cavity side wall at yaw angle $=45^{\circ}-75^{\circ}$ and a strong vortical structure forms along the cavity axis. It was shown by the mean pressure measurements that for cavities with aspect ratio larger than $\mathrm{W} / \mathrm{D}=2$, at yaw angle $=30^{\circ}-60^{\circ}$, steep pressure gradients occur. Also, a region of high stagnation pressure at the downstream cavity side walls shows the shear layer impingement. Figure 2-9 illustrates a schematic model of flow field inside a yawed cavity. By a detailed study of the unsteady pressure field for several cavity depths, it was found that strong instabilities only occur if the vortical structure inside the cavity is well established. Shallower cavities $(h / D<0.5)$ produce lower instability magnitude at all aspect ratios and yaw angles and the highest instabilities occur at $\mathrm{h} / \mathrm{D}=0.57-0.67$. It was observed that the magnitudes or frequencies do not change significantly with boundary layer thickness. He concluded that the extent of flow entrainment into the cavity is the main parameter that influences the mean and unsteady pressures as well as the drag coefficient. Also, it was shown that the interactions between the separated shear layer and the cavity base, which change with yaw angles and cavity depth, are the main reasons for changes in the mean and unsteady pressure field [50].

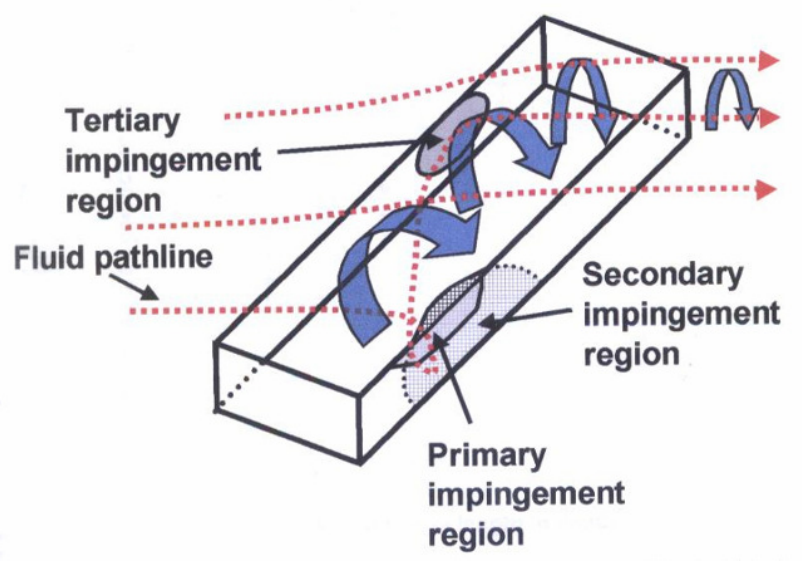

Figure 2-9 Schematic model of aerodynamic phenomena resulting in vorticity shedding [49]

\subsection{Different Cavity Planforms}

The flow associated with cavities with curved walls (elliptical and circular cavities) is similar in certain aspects to that of the rectangular cavities. However, some differences exist in flow field. Most of the investigations related to the cavity planform geometry are 
limited to the effect of cavity planform shape on drag [3], and only a few focus on the flow inside and downstream of the cavity. The earliest investigations led to the determination of the effect on drag of cavity depth and aspect ratio for elliptical cavities [3]. A peak in the drag was found, due to flow asymmetry, at certain cavity depths ( $h / D \cong 0.5$ ) for a cavity with a circular planform, which is a special case of the elliptical planform cavity with a 1:1 aspect ratio. It was also noted that increasing the aspect ratio decreased the resulting drag for $\mathrm{h} / \mathrm{D}<0.8$, while for deeper cavities, the drag value was almost constant [3]. Through mean pressure measurements on circular cavity surfaces, it was shown that a stable captive vortex exists inside the circular cavity for most depths. But, again, for $h / D \cong 0.5$ the pressure distribution was found to be highly asymmetric [51], [52] and vortices were shed from the cavity into the external flow [53].

The work of Haigermoser et al. [54] is an example of application of the latest nonintrusive flow measurement techniques in the case of a three-dimensional cavity with a circular planform and $\mathrm{h} / \mathrm{D}=0.5$, exposed to a turbulent upstream boundary layer. They used stereoscopic PIV measurements in the vertical plane $(\mathrm{z}=0)$ and multiple horizontal planes, as well as tomographic PIV in the volume of the cavity, to describe the threedimensional flow inside the cavity. In the vertical plane, the flow was found to be dominated by a recirculation zone, with the locations of the maximum velocity fluctuations close to those of rectangular cavities. An important phenomenon observed, thanks to the ability of their measurement techniques to cover the three-dimensional volume of the cavity, was the asymmetric structure of the vortex associated with the circulation zone. While care was taken to ensure symmetry in the experimental setup, the flow was found to enter the cavity volume on one side, circulate, and then exit the volume on the other side. Increased turbulence intensity was found where the flow exited the cavity and interacted with the freestream. A similar asymmetric behaviour has been reported by Dybenko and Savory for a circular cavity with $\mathrm{h} / \mathrm{D}=0.5$, at $\operatorname{Re}_{D}=1.3 \times 10^{5}$ [43].

Elliptical cavities yawed to the flow were first investigated by Savory and Toy [5], who noticed an increase in drag for certain yaw angles. Also, Hering and Savory studied flow regimes for a 2:1 aspect ratio elliptical cavity with various yaw angles and cavity depths, 
and categorized the elliptical cavity flow regimes into several different types (Figure 2-10) [6]. Although some similarities have been found between rectangular and elliptical cavity flow regimes, the effect of the planform radius of curvature is not fully understood and so far only a limited number of yaw angles have been investigated.

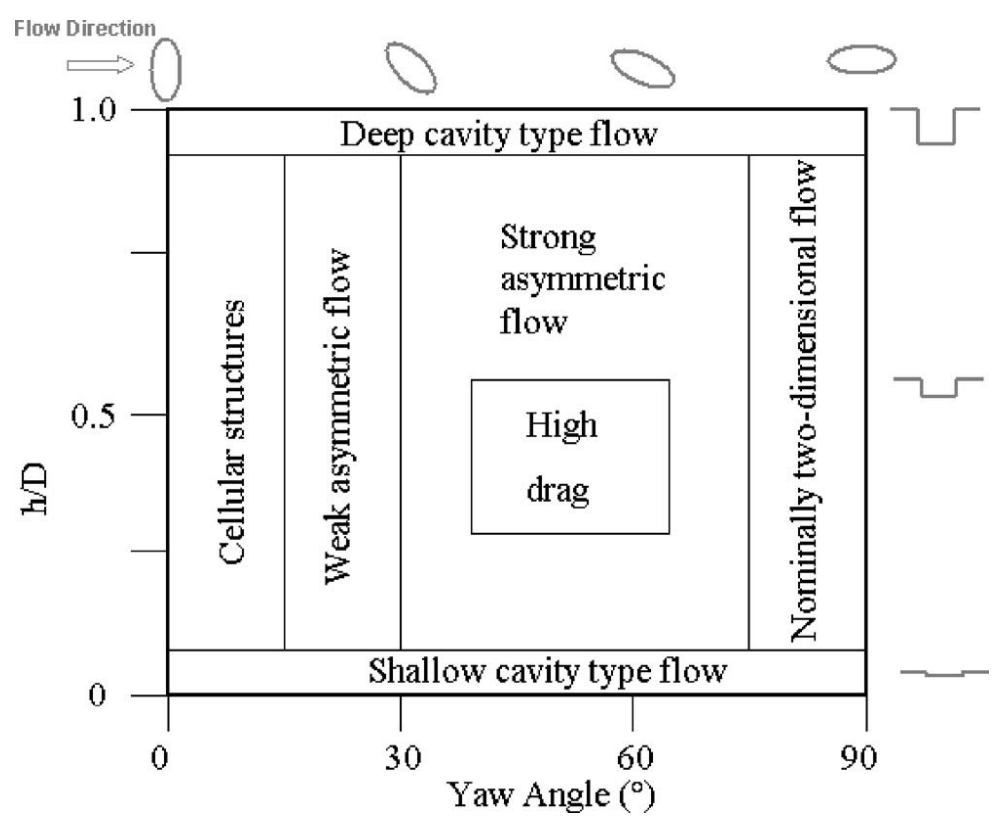

Figure 2-10 Resulting flow regimes of yawed elliptical cavities [6]

Due to the complexity of the PIV measurements through curved surfaces, no PIV results for elliptical cavities have been reported so far. One of the solutions to overcome this problem is gaining optical access to the interior of the cavity by setting the camera at an angle, similar to the experimental setup used by Haigermoser et al. [54]. The other solution is to compensate the effect of the image distortion caused by the surface by quantifying the distortion using a reference image.

\subsection{Drag of Cavities}

The effects of different cavity geometries and approaching boundary layer conditions on drag coefficient have been studied by many researchers. Most of these drag data have been derived from force balance measurements which can not be used to specify the 
cavity flow regimes. Only a few researchers have used surface pressure distribution measurements to extract the aerodynamic forces [48].

Tani et al. showed that open cavities have lower drag compared to the closed ones [12]. In the case of open cavities the lower part of free shear layer, which has a lower velocity, spans the cavity opening and the maximum pressure on the downstream wall is found at the lip. However, for the closed cavities the upstream wall acts like a forward facing step and not only faces higher velocity, but also the stagnation point is located slightly below the lip. As a result, the pressure on the downstream wall is higher in the case of shallower cavities, which means a higher drag.

They have also shown that for the step case, as the boundary layer thickness is reduced, the pressure rise due to flow reattachment increases [12]. The result of a later study by Plentovich with two different approaching boundary layer thicknesses, $\delta / \mathrm{D}=0.021$ and 0.014 , show that when the boundary layer entering a cavity is thinner, the pressure distribution becomes more positive in the aft region and more negative downstream of the cavity [18]. The reason is that, in case of thinner boundary layer, a higher velocity flow is entrained into the cavity. Therefore, when the boundary layer thickness decreases, the drag of the cavity increases.

This phenomenon was also explained by Haugen and Dhanak's analytical model for a two-dimensional rectangular cavity exposed to a turbulent oncoming boundary layer [55]. They proposed a model in which the drag of the cavity is proportional to the average shear stress $\bar{\tau}$ at the mean dividing streamline (i.e., $\mathrm{y}=0$ ). When the boundary layer thickness increases, the shear layer entrainment thickness increases as well (less curvature), and as a result the velocity gradient $\left(\frac{\partial u}{\partial y}\right)$ and thereby $\bar{\tau}$ decreases and one can conclude based on this proposed model that drag will also be lower. The model has been verified against experimental data obtained by measuring the difference between the integrated mean values of pressure coefficient over the upstream and downstream walls of the cavity. 
Gaudet and Winter studied the drag of rectangular and circular cavities [56]. They suggested a model that the separated shear layer along the cavity opening was the reason for the complexity of the cavity flow regimes. In their model, the cavity drag is a function of the effective shear stress $\left(\tau^{\prime}\right)$ arising from the separated shear layer. For rectangular cavities they used the following equation to make the effective shear stress nondimensional, using the approaching boundary layer shear stress $(\tau)$ and related that to the net drag coefficient $\left(\frac{\Delta C_{D}}{C_{f}}\right)$ :

$$
\frac{\Delta C_{D}}{C_{f}}=\frac{\tau^{\prime}-\tau}{\tau}
$$

where $\Delta C_{D}$ is the incremental drag coefficient due to the presence of the cavity and $C_{f}$ is the local skin friction coefficient of the boundary layer. Using the normalized drag coefficient makes it possible to fairly compare experimental data with different approaching boundary layer characteristic. However, the effects of particular aspects of the cavity flow, such as cavity planform shape, aspect ratio, h/D and also Reynolds and Mach numbers should be considered.

In the case of circular cavities the model is more complicated and $\frac{\Delta C_{D}}{C_{f}}$ is a function of geometrical specifications, Mach number, viscosity, and friction velocity. So, the following model has been proposed for circular cavities:

$$
\frac{\Delta C_{D}}{C_{f}}=A\left(\frac{u_{*} D}{v}\right)^{B}
$$

where $u_{*}$ is the friction velocity, $D$ is the diameter of the cavity, $v$ is the viscosity of the fluid, $A$ is a function of $\mathrm{h} / \mathrm{D}$ and Mach number, and $B$ is a constant for any given Mach number.

Young and Paterson provided comprehensive drag data which can be used for design purposes [57]. They used previous researchers' experimental data to extract a mean curve 
for variation of normalized drag coefficient with h/W. As can be seen in Figure 2-11, shallower cavities produce larger drag. Also, increasing the aspect ratio decreases the resulting drag for shallow cavities. But for deeper cavities $(\mathrm{h} / \mathrm{W}>1)$ the drag coefficient does not vary with $\mathrm{h} / \mathrm{D}$. The same trend was observed for elliptical cavities as well except for the particular case of $\mathrm{D} / \mathrm{W}=1$ (circular cavity) which shows a peak in drag for $\mathrm{h} / \mathrm{D}=$ 0.5, as was discussed in the previous section (Figure 2-12).

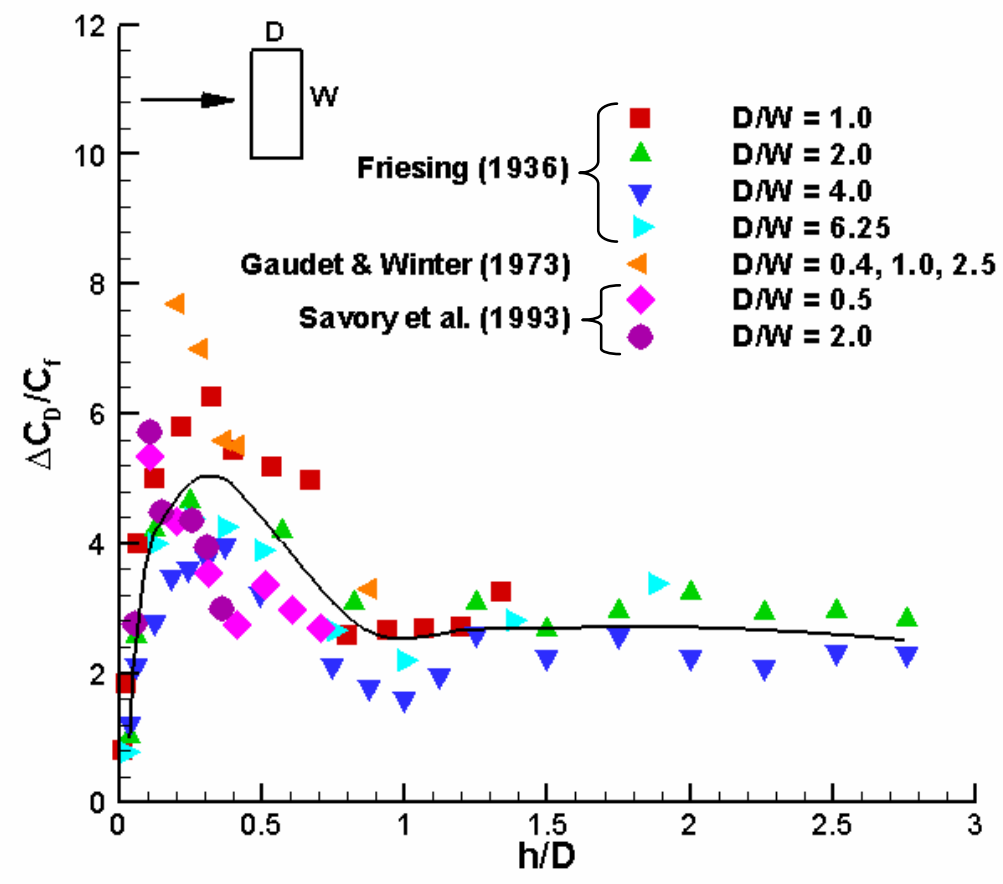

Figure 2-11 Variation of normalized drag coefficient with $\mathbf{h} / \mathrm{D}$ for rectangular cavities at $0^{\circ}$ yaw angle (The line is added to visualize the general trend.)

The effects of yaw angle on cavity drag for rectangular cavities were explained in section 2.6. In the case of elliptical cavities, Hering produced a contour plot which illustrates variation of the normalized drag coefficient with h/D and yaw angle (Figure 2-13) [58]. The general trend is similar to that found by Savory et al. for rectangular cavities [48] and the maximum drag occurs for $\mathrm{h} / \mathrm{D} \cong 0.5$ and yaw angle $=45^{\circ}-60^{\circ}$ which can be due to highly asymmetric flow patterns in these cases. 


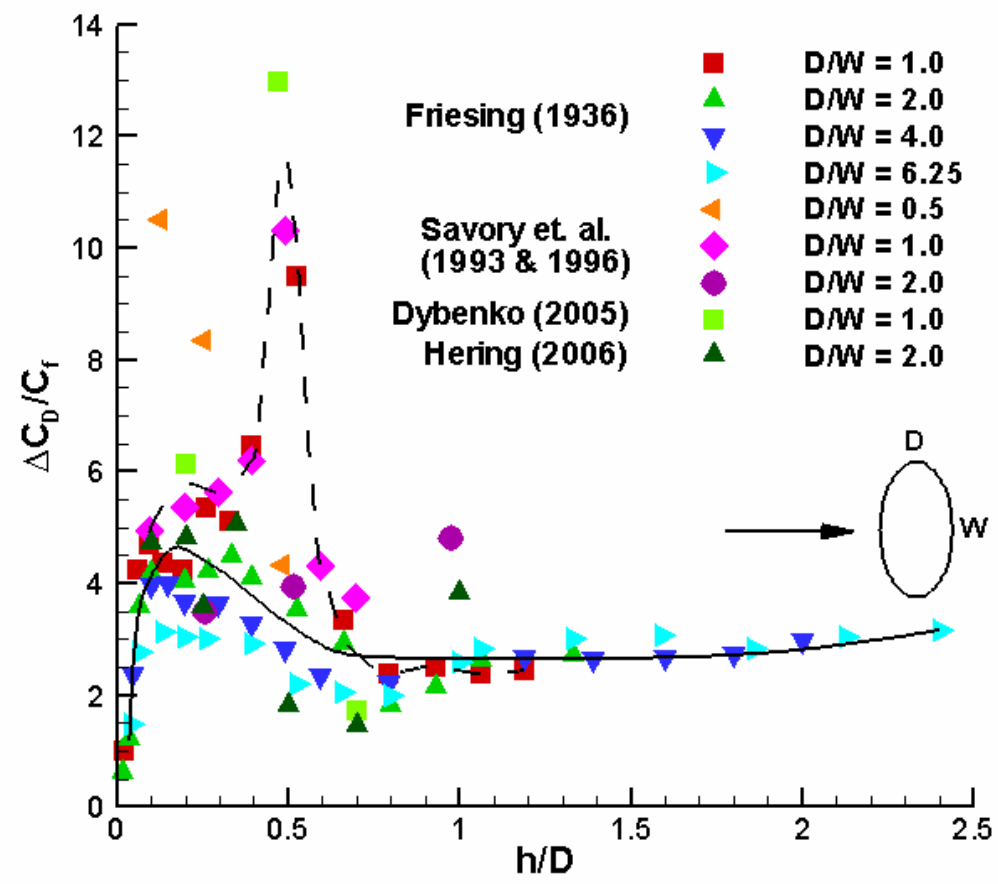

Figure 2-12 Variation of normalized drag coefficient with $\mathrm{h} / \mathrm{D}$ for elliptical cavities at $0^{\circ}$ yaw angle (The lines are added to visualize the general trend for elliptical (solid line) and circular (dashed line) planform shape.)

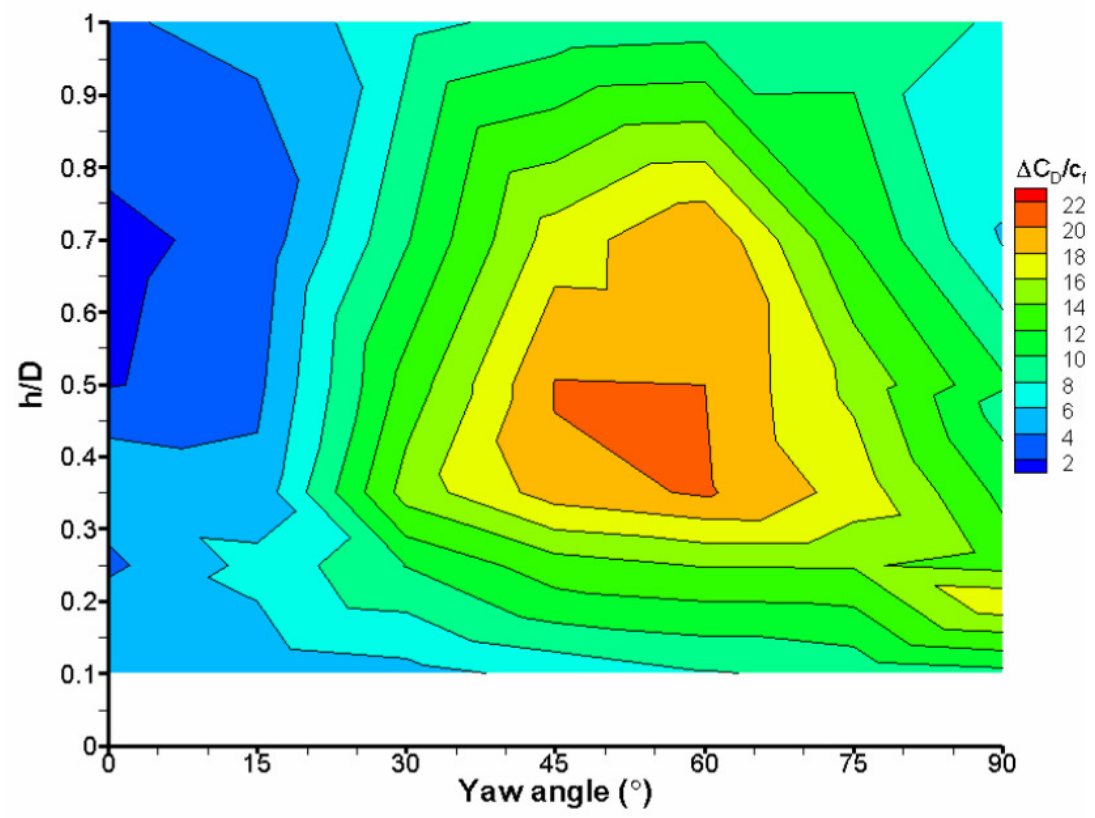

Figure 2-13 Effect of yaw angle and depth on the normalized drag coefficient of an elliptical cavity $(\mathrm{W} / \mathrm{D}=2)[58]$ 


\subsection{Numerical Simulations of Cavity Flows}

Considering the highly three-dimensional flow inside the cavities and the challenges of experimental measurements in the enclosed space of a cavity, researchers have used numerical analysis as a tool to model and predict the flow in a cavity, and to avoid the experimental complexities. With modern advances in computing techniques and computing power, Computational Fluid Dynamics (CFD) methods are now helpful as an aid to the understanding of the flow processes involved in the unsteady aerodynamics of cavities. Numerical analysis also makes it possible to examine multiple combinations of the parameters discussed in the previous sections, for which experimental analysis can be time consuming and costly.

Cavity flow simulations have been under development and there are many challenges to overcome. Two main problems are meshing and turbulence modeling which can be interdependent, thus adding the complexity. The simulation can be affected by many other factors such as discretization technique, flux representation, boundary conditions, and time stepping [14].

Direct Numerical Simulation (DNS), with no requirement for turbulence modeling would be the best approach, but the computational effort is prohibitive. Therefore, different degrees of turbulence modeling, ranging from Large Eddy Simulation (LES) or Detached Eddy Simulation (DES) to Reynolds Averaged Navier Stokes (RANS) solutions using an assortment of two-equation (e.g. $k$ - $\omega$ or $k-\varepsilon$ ) or algebraic turbulence models are used. LES and DES methods require more computing power than RANS based two-equation methods. Although recent LES-based methods are better for modeling cavity static forces (steady or time averaged pressures) and dynamic forces (unsteady pressures), RANS based methods should not be dismissed, as they are less computationally demanding and have been used with some success. DES is a hybrid RANS-LES approach with benefits from both methods in difficult areas, such as in the free shear layer (LES) and lower computational demands in other suitable areas such as approaching boundary layer (RANS) [14]. 
The backward facing step problem served as a precedent for the numerical analysis of cavity flows. Due to the simple geometry and availability of experimental data, the backward facing step has become a benchmark and validation test problem for CFD simulations. Several researches simulated the laminar flow over two-dimensional and three-dimensional backward facing steps, using different methods and algorithms. The two-dimensional numerical simulations usually show good agreement with the experimental results for $\operatorname{Re}<400$, but underestimate the reattachment length for $\operatorname{Re}>400$ [59], [60]. The reason for the discrepancy is the three-dimensionality of the flow as has been observed in experiments before [61]. Le et al. investigated the three-dimensional turbulent flow over a backward facing step, using DNS at $\operatorname{Re}=5100$. The results were in very good agreement (within 3\% error) with experimental data in predicting reattachment length and skin friction coefficient [62]. Later investigators showed the complementary nature of the experimental and numerical simulations. They showed that the main advantage of experiment was the relative easiness in measurement of unsteady flow behaviour and on the other hand numerical simulation is capable of providing spatiotemporal details of the flow which cannot be easily obtained in the experiment [63].

The numerical analysis of cavity flows begun in the 1960s. These studies were mainly focusing on flow inside two or three-dimensional lid-driven cavities [64]. In lid-driven cavity flow simulation, the interaction between the shear layer and the recirculation flow and the effects of cavity flow on the downstream are not considered and only the flow field inside the cavity is modeled [65]. Therefore, the results of these simulations are different from the real cavity flow phenomena.

Yao et al. simulated the unsteady incompressible laminar flow past three-dimensional open rectangular cavities using a finite difference scheme for solving the unsteady Navier-Stokes equations. They studied different inflow conditions, different Reynolds numbers, and h/D. They observed that by increasing the Reynolds number, the cavity flow becomes extremely unsteady and it affects the boundary layer downstream of the cavity. At high Reynolds numbers, longitudinal vortex structures were also captured which can not be simulated by a two-dimensional model. Also, it was observed that as 
the thickness of the boundary layer decreases, the flow becomes more unstable in terms of larger oscillations in the shear layer [64].

The other main focus of cavity flow numerical simulations in the last two decades has been on flow oscillations. Early numerical studies of compressible cavity flows have used the two-dimensional unsteady RANS equations with a $k-\varepsilon$ turbulence model [66] while the compressible turbulence models for predicting separated oscillating flows and, especially, their radiated acoustic field have not been found to be effective [67]. Rizzeta simulated supersonic flow over a three-dimensional cavity using the RANS method and a simple algebraic closure model for the turbulent viscosity coefficient and found that, even for the three-dimensional method, discrepancies in comparison with experimental data exist. He suggested several possible reasons for these differences, including inadequate numerical resolution, numerical damping, the use of the eddy viscosity model, and discrepancy between the calculated and physical upstream shear layer profiles [68]. Later he tried the LES method and realized that this model produces more precise results [69]. Rowley et. al. [67] showed DNS at low Reynolds numbers presents a more accurate method to predict the details of oscillation modes consistent with the acoustic feedback mechanism of Rossiter [38]. Also, it was shown that the wake mode oscillation is similar to that reported by Gharib \& Roshko [43]. They found that the frequency of oscillations in the wake mode is independent of Mach number. In the wake mode, a significant backflow was present inside the cavity, which led to an absolute instability and, as a result, the feedback mechanism switched to the wake mode [67].

Ideally, a numerical study of a three-dimensional cavity should include the effects of the unsteady flow, and should use an approach to the issue of turbulence which would account for the effects of turbulence in all scales on the cavity flow. An example of such a study in the case of a circular cavity is Grottadaurea and Rona's work [70]. To model the effects of turbulence, they have used Detached Eddy Simulation, which is a combination of a RANS turbulence model for the near wall region and LES for the rest of the solution domain. Such a numerical analysis requires a large amount of computational resources, which has been provided by a national high performance computational network in case of Grottadaurea and Rona. This computational approach may not be 
feasible when multiple simulations are needed to study the effects of several combinations of flow and geometric parameters.

Zdanski et al. numerically simulated both laminar and turbulent flows over shallow rectangular cavities to model solar collectors with wind barriers [71]. They used the RANS equations with a $k-\varepsilon$ turbulence model. They studied the influence of cavity aspect ratio, turbulence level of the oncoming flow and Reynolds number. They have argued that although the instantaneous flow inside cavities plays an important role, the mean flow, which can be modeled through a steady simulation, can predict the pressure coefficient distribution with a difference of 0.03 or less with the experimental data. They used this comparison to consolidate their code validation [71]. Although steady simulations of the mean flow do not provide any information about flow dynamics, they allow the analysis to be carried out within a reasonable frame of time and computational resources. Therefore, steady simulations can be considered as a useful approach when several simulations involving combinations of flow and geometric parameters are required.

In another study conducted by Durteste, it has been shown that the quasi-steady assumption for cavity flow can be considered. She showed that it is possible to define a dividing streamline that separates the flow which enters the cavity from that which does not. Since the boundary layer is turbulent, some exchange of fluid occurs but it can be neglected. Therefore, although cavity flows are unsteady, it has been possible to identify a typical steady flow configuration to reduce the cost and time of computations. To confirm this, she compared the drag coefficient calculated from the steady simulation with the mean drag coefficient from the unsteady calculation and showed that the results were similar [72].

Czech used a commercial CFD package (FLUENT) to simulate the flow over yawed rectangular cavities [49]. He used time averaged turbulent flow for this simulation and the $k-\varepsilon$ model was chosen due to grid and computational limitations. The resulting drag coefficient data were compared with experimental data and were found to be within $10 \%$ of the experimental values. Pathlines, velocity field and turbulent kinetic energy inside 
the cavities were studied through these simulations which provided a valuable insight into the development of vortices in the cavity. The overall mean pressure field was predicted reasonably well, but there were quantitative differences (10\%) in drag coefficient calculations for some yaw angles. He suggested that those differences might arise due to disability of the $k-\varepsilon$ model to predict the separated flows accurately, grid distribution, or the use of wall functions.

Of the entire body of research dealing with the numerical analysis of cavity flows, only a small fraction is dedicated to the case of flow in a circular cavity. Beside the works of Grottadaurea and Rona [70] which was mentioned before, Hering et al. [73] and Marsden et al. [46] also conducted CFD studies of flow in and around circular cavities. Hering et al. used FLUENT to simulate turbulent flow over circular cavities with varying depths. They used time averaged turbulent flow and $k-\varepsilon$ model as well. The flow physics and qualitative flow behaviour for most of cases were simulated with reasonably good quality, but the pressure coefficient values were underestimated. Also, the flow asymmetry for the special case of $\mathrm{h} / \mathrm{D}=0.47$ was not as strong as predicted by the experimental results and this resulted in drag coefficient underestimation.

Marsden et al.'s research was mostly focused on acoustic radiation of circular cavities [74]. They used LES to simulate the turbulent flow over circular cavities with $h / D=0.5$ and 1.0 at Mach number $=0.26$. For the deeper cavity they observed a symmetrical pressure distribution on cavity walls, while the shallower cavity had an asymmetric mean flow field. Unlike Dybenko and Savory's results [53], for $\mathrm{h} / \mathrm{D}=0.5$, a tonal emission was not observed around $\mathrm{St}_{\mathrm{D}}=0.41$ for neither their experimental tests nor numerical simulations. Dybenko and Savory suggested that this mechanism and its related peak could be associated with the asymmetric mean flow. Marsden et al. concluded since they did not observe this peak and they still captured asymmetric flow, this can not be the main reason for the flow asymmetry.

The details of the flow in and around elliptical cavities are substantially different from other types of cavity, as described in section 2.7. However, very little experimental data, and almost no numerical analysis has been reported in the case of elliptical cavities. 
Numerical simulations, when based on a reliable numerical model, can lead to a better understanding of the physics of flow in and around cavities, by providing a threedimensional set of data with the appropriate spatial and temporal accuracy, which can be manipulated to identify flow phenomena such as generation and shedding of vortices, and to estimate the overall aerodynamic characteristics. Confidence in the numerical model can be gained by employing the techniques and methods that have been proven to provide acceptable results in the literature [49], [73], and by validating the model against the existing experimental data and tailoring the numerical model to replicate these data accurately. Once the appropriate numerical model is established, the simulations can be extended to the cases where no previous experimental numerical or experimental data are available, including various combinations of the governing parameters such as the oncoming boundary layer, $\mathrm{h} / \mathrm{D}$ ratio, and yaw angle. Such a systematic study can provide better insight into the flow in and around elliptical cavities, and extend the knowledge base in this area.

The review of previous research involving numerical simulation of cavity flow presented herein indicates that in order to achieve a high quality prediction of the flow, the unsteady effects should be accounted for in the simulation, and that the most accurate approach to the issue of turbulence should be implemented. Considering the state-of-the-art in CFD, this usually means a three-dimensional, unsteady simulation, with LES or even DNS employed for turbulence treatment. However, this level of sophistication may not be achievable for all researchers, due to limitations in computer resources. These limitations become especially prohibitive when several simulations involving various combinations of geometric and flow parameters are to be studied in order to establish a trend, to describe the effects of these phenomena on cavity flows.

The usual practice in this situation, as implemented by Czech [50], Zdanski et al. [71], Durteste [72] and Hering [73] is to settle for a simpler numerical model that is still able to predict the principal flow phenomena, which means the use of the best available RANS turbulence model instead of LES or DNS, and focusing on the mean flow characteristics through steady simulations. The same practice has been implemented in the present research. However, unlike the above-mentioned researchers, who used turbulence models 
limited to isotropic turbulence (i.e. $k-\varepsilon$ ), the more advanced Reynolds Stress Model (RSM), which is able to account for non-isotropic turbulence has been utilized in the present research, and the individual Reynolds stress components have been modeled using input profiles based on actual wind tunnel measurements.

\subsection{Summary}

In this chapter, the important features of cavity flows were introduced and discussed. It was shown that the cavity flow regime varies with geometry (depth to length ratio, aspect ratio, and planform shape), yaw angle, Reynolds number (laminar or turbulent flow), approaching boundary layer parameters, and Mach number. Cavity flows were classified into open, transient, and closed regimes, and their main features were defined. The threedimensional nature of flow inside the cavities was discussed.

The different types of cavity flow oscillations including fluid-elastic, fluid-resonant, and fluid-dynamic, were introduced, with the main focus on the feedback resonance mechanism. Some empirical models for prediction of the resonant frequency and their validity for different flow conditions and planform shapes were discussed. Also, the effects of the approaching flow conditions (Re and Mach numbers), as well as the effect of boundary layer thickness on cavity resonance were described.

Afterwards, the effects of yaw angle and different planform shapes, including rectangular, circular, and elliptical planforms, on flow field asymmetry were explained. The drag of cavities and the effects of the previously mentioned parameters including the cavity type, geometry, planform shape, yaw angle, etc. on drag was studied. Also, a nondimensional parameter, normalized drag, was introduced, which enables researchers to compare the drag results regardless of the approaching boundary layer characteristics.

As discussed in detail in Chapters 3 and 4, experimental measurements of the flow field using Particle Image Velocimetry (PIV) and numerical simulations have been utilized in the present study as the two principal methods to investigate the flow inside and around cavities with elliptical planforms, with various yaw angles and depths. 
The review of the studies involving PIV measurement of flow in cavities highlights the capabilities of this technique to provide valuable information on flow structure (as specified by the characteristics of shear layer, and the arrangement and position of vortices in the cavity), the spatial distribution and growth of velocity fluctuations and turbulent stresses in the cavity and the dynamic behaviour of the cavity flow. This was complemented by an overview of the effects of parameters including boundary layer state, Reynolds number, and cavity depth, on the above-mentioned characteristics of the flow.

Finally, different numerical simulations and the respective simulation techniques including turbulence modeling methods, steady/unsteady modeling, etc. were discussed. Based on the findings of the previous studies involving numerical simulations, and considering the limitations of computational resources and the numerous combinations of geometric and flow parameters involved in the present study, steady simulations with RSM as the turbulence model are selected for the present research. Although such numerical simulations have their own limitations, they have previously been shown to be able to provide valuable insights into cavity flow phenomena associated with the mean flow [50], [71], [72], and [73].

The results of the previous research work on the flow in cavities, which have been reviewed in this chapter, indicate that the flow structure, pressure distribution, and drag of cavities are affected by a number of principal parameters. These parameters can be divided into two groups: geometric parameters, which include the presence of sidewalls and curvature, cavity depth, yaw angle and planform shape and flow-related parameters, including Reynolds number and boundary layer thickness and state. The effects of these parameters will be summarized in the following paragraphs:

\section{- Sidewalls and curvature}

While the flow in three-dimensional cavities is dominated by the same principal phenomena that dominate the flow in two-dimensional cavities (shear layer separation, reattachment, and impingement, and formation of vortices inside the cavity), there are significant differences between the flow in two and three- 
dimensional cavities. These differences originate from the effects of cavity sidewalls, and in the case of cavities with circular and elliptical planforms, the curvature of the cavity walls. The presence of sidewalls and curvature of the cavity walls lead to variations in the position and strength of the vortices across the span of the cavity, emergence of flow components in the spanwise direction, and additional vortical structures resulting from the interaction of the side walls and the freestream. These effects, in turn, result in spanwise variations of pressure distribution on the cavity surfaces. In addition to these effects, the flow in cavities is also affected by the formation of "cellular" structures across the span, even for large aspect ratio cavities. This phenomenon has been documented for rectangular cavities, and the number of these structures is found to be dependent on the geometric proportions of the cavity, with a larger number of structures for cavities with larger aspect ratio. Previous pressure measurements have shown indications of this phenomenon in elliptical cavities.

\section{- Cavity depth}

Depending on the cavity depth, the flow inside the cavity follows certain distinct changes in the flow structure, represented by "closed", "transitional" and "open" regimes. When the cavity depth is increased, a transition of flow regime from "closed" to "transitional" and then to "open" is observed. The definition of these regimes originates from two-dimensional cavities. However, three-dimensional cavities with various planforms have been found to follow the same transition of flow structures. The drag of cavities in the "closed" flow regime has been found to be larger than that of "open" cavities.

\section{- Planform shape}

Previous studies on cavity flow include rectangular, circular, and elliptical planforms. The studies on circular and elliptical cavities mainly focus on pressure distribution and drag, rather than flow structure. These studies indicate that 
cavities with circular and elliptical planforms have highly asymmetric flows at certain yaw angles, which lead to drag forces larger than those of comparable rectangular cavities. Curvature of the cavity sidewalls results in additional threedimensional effects on the flow, which have not been documented systematically.

\section{- Yaw angle}

Increasing yaw angle has been found to lead to flow asymmetry, for both rectangular and elliptical cavities. The extent of this asymmetry, however, depends on the geometric parameters and yaw angle. At moderate yaw angles (up to $30^{\circ}$ ) the cavity flow characteristics remain similar to the cavity aligned with the flow. However, when the yaw angle reaches $45^{\circ}$, a sudden increase in cavity drag is observed. This behaviour is accompanied by the departure of the vortex from the downstream part of the cavity and formation of a trailing vortex which affects the flow downstream of the cavity. This behaviour is more pronounced in deep cavities, and the flow in shallow cavities is found to retain its similarities to the flow in a cavity aligned with the flow, even at high yaw angles. Previous studies involving rectangular and elliptical cavities indicate a maximum drag for cavities with intermediate depth, at yaw angles of $45^{\circ}$ and $60^{\circ}$.

\section{- Thickness and state of the boundary layer}

Another parameter affecting the cavity flow is the thickness and state of the boundary layer upstream of the cavity. A thicker boundary layer leads to lower velocities being entrained in the cavity and, therefore, lower levels of positive pressure in the cavity. As a result, a cavity exposed to a thicker boundary layer has a lower drag. Parameters related to the velocity gradient in the separated shear layer, such as skin friction coefficient and shear stress components inside the cavity, are also found to have lower levels when the upstream boundary layer is thicker. The growth of the separated shear layer is larger for a turbulent upstream boundary layer, leading to more momentum being entrained in the cavity. For 
open cavities, it has been shown that for certain combinations of upstream flow conditions (including Reynolds number and boundary layer parameters) and cavity length, a feedback resonance mechanism is observed in the shear layer, as described in detail in the literature review.

\section{- Reynolds number}

Reynolds number also affects the flow in the cavity. In all of the studies reviewed in the previous sections, dimensionless velocity fluctuations and Reynolds stress components were found to increase with increasing Reynolds number, and towards the downstream wall. For a given state of boundary layer (laminar or turbulent), the growth of the shear layer has been found to be independent of Reynolds number in most studies, and follow a linear trend with increasing distance from the upstream edge of the cavity. The size and position of the vortices inside the cavity is also affected by Reynolds number. While a distinct secondary recirculation zone is observed near the upstream wall at lower Reynolds numbers, for cavities exposed to turbulent boundary layer the primary recirculation zone grows and the secondary recirculation zone diminishes in size as Reynolds number increases.

The review presented in this chapter indicates that the physics of the flow associated with elliptical cavities has not been completely determined, because of the limited number of previous studies in this area. The gaps in the knowledge on the flow associated with elliptical cavities, have been described in Chapter 1, and form a basis for definition of the hypotheses and objectives of the present research. 


\section{Numerical Simulation}

\subsection{Introduction}

In recent years, the use of Computational Fluid Dynamics (CFD) codes for flow simulation and related calculations has increased significantly. Numerical simulations help researchers to understand more details regarding the flow field. Different techniques (including different turbulence modeling methods, steady/unsteady, wall functions, etc.) are being used for these simulations according to the nature of the flow, its complexity and the computational resources. As was explained in section 2.9, cavity flow simulations have been carried out since the 1960s, with the main focus being on rectangular cavities.

In this chapter, the governing equations of fluid flow and the turbulence models are introduced. Then, the computational domain, boundary conditions and computational grid are explained. These sections are followed by a description of the computational implementation chosen in this study.

\subsection{Governing Equations}

Computational Fluid Dynamics (CFD) is based on the fundamental governing equations of fluid dynamics, including the continuity, momentum, and energy equations. The energy conservation equation is only solved for flows involving heat transfer. Otherwise, only mass and momentum conservation (Navier-Stokes) equations are solved.

As mentioned in Chapter 2, Direct Numerical Simulation (DNS), with no requirement for turbulence modeling would be the best approach to simulate the flow. However, in the case of complex geometries and high Reynolds number turbulent flows, due to the computational cost, time-dependent direct solution of the Navier-Stokes equation is not feasible. There are two main methods which do not require the direct simulation of the small scale turbulent fluctuations. These methods are: Large Eddy Simulation (LES) and Reynolds Averaged Navier-Stokes (RANS). Statistically unsteady (or non-stationary) flows can be treated using RANS method. This is sometimes referred to as URANS. There is nothing inherent in Reynolds averaging to preclude the use of RANS models for 
unsteady flows, but the turbulence models used to close the equations are valid only as long as the time over which changes in the mean occur is large compared to the time scales of the turbulent motion. All of the above mentioned methods introduce additional terms in the governing equations that need to be modeled in order to achieve a closure for the unknowns [75].

In the LES method, large eddies are resolved in a time-dependent simulation using the filtered Navier-Stokes equations. Filtering means removing eddies that are smaller than the size of the filter, which is usually taken as the mesh size. Since large scale motions are generally more energetic and effective than the small scale ones, this kind of approach make sense. Due to the mesh size and the number of cells (especially in near wall regions), LES for high Reynolds number flows requires a significant amount of computational resources [75].

In Reynolds averaged approaches to turbulence, all of the unsteadiness is averaged out and the nonlinearity of the Navier-Stokes equations gives rise to terms that must be modeled. Therefore, this method greatly reduces the required computational resources and is a proper approach for applications in which the fine spatial and temporal scales related to turbulence are not required to be resolved. In this method, each variable in the Navier-Stokes equations is divided to time-averaged and fluctuation components. For the velocity components:

$$
u_{i}=\bar{u}_{i}+u_{i}^{\prime}
$$

where $\bar{u}_{i}$ is the mean and $u_{i}^{\prime}$ is the fluctuating velocity component.

By substituting all of the variables into the instantaneous continuity and momentum equations for incompressible flow and taking an ensemble average (represented by the overbar), they can be written as:

$$
\frac{\partial \bar{u}_{i}}{\partial x_{i}}=0
$$




$$
\frac{\partial}{\partial t}\left(\bar{u}_{i}\right)+\frac{\partial}{\partial x_{j}}\left(\bar{u}_{i} \bar{u}_{j}\right)=-\frac{1}{\rho} \frac{\partial \bar{p}}{\partial x_{i}}+\frac{\partial}{\partial x_{j}}\left[v\left(\frac{\partial \bar{u}_{i}}{\partial x_{j}}+\frac{\partial \bar{u}_{j}}{\partial x_{i}}\right)\right]+\frac{\partial}{\partial x_{j}}\left(-\overline{u_{i}^{\prime} u_{j}^{\prime}}\right)
$$

These equations are called the Reynolds Averaged Navier-Stokes (RANS) equations. The variables in these equations represent ensemble averaged values, however, an additional term, which represents the effect of turbulence, appears in the equations. This term, which is known as the Reynolds stress $\left(-\rho \overline{u_{i}^{\prime} u_{j}^{\prime}}\right)$, must be modeled in order to close the equations [76]. A simple method is that the effect of turbulence can be represented as an increased viscosity. This approach is called Boussinesq hypothesis or the eddy viscosity model:

$$
-\rho \overline{u_{i}^{\prime} u_{j}^{\prime}}=\mu_{t}\left(\frac{\partial \bar{u}_{i}}{\partial x_{j}}+\frac{\partial \bar{u}_{j}}{\partial x_{i}}\right)-\frac{2}{3} \rho \delta_{i j} k
$$

where $\mu_{t}$ is the turbulence viscosity and $k$ is the turbulence kinetic energy which can be calculated from:

$$
k=\frac{1}{2} \overline{u_{i}^{\prime} u_{i}^{\prime}}
$$

The Boussinesq hypothesis is used in the Spalart-Allmaras model [77], $k$ - $\varepsilon$ models [78], and $k$ - $\omega$ models [79]. The disadvantage of this approach is that it assumes $\mu_{t}$ is an isotropic scalar quantity resembling a fluid property, which is not strictly true, since it depends on the local turbulence velocity and length scales. In three-dimensional flows, the Reynolds stress and strain rate will not be related in such a simple way. Both measurements and simulations show that $\mu_{t}$ becomes a tensor quantity and is no longer a scalar [76].

The alternative approach, used in the Reynolds Stress Model (RSM), is to solve transport equations for each of the terms in the Reynolds stress tensor [75]. The exact transport equations for the transport of the Reynolds stresses, $-\rho \overline{u_{i}^{\prime} u_{j}^{\prime}}$, may be written as follows: 


$$
\begin{aligned}
& \underbrace{\frac{\partial}{\partial t}\left(\rho \overline{u_{i}^{\prime} u_{j}^{\prime}}\right)}_{\text {Local Time Derivative }}+\underbrace{\frac{\partial}{\partial x_{k}}\left(\rho u_{k} \overline{u_{i}^{\prime} u_{j}^{\prime}}\right)}_{C_{i j} \equiv \text { Convection }}=\underbrace{\left.-\frac{\partial}{\partial x_{k}}\left[\rho \overline{u_{i}^{\prime} u_{j}^{\prime} u_{k}^{\prime}}+\overline{p^{\prime}\left(\delta_{k j} u_{i}^{\prime}+\delta_{i k} u_{j}^{\prime}\right.}\right)\right]}_{D_{T, i j} \equiv \text { Turbulent Diffusion }}+\underbrace{\frac{\partial}{\partial x_{k}}\left[\mu \frac{\partial}{\partial x_{k}}\left(\overline{u_{i}^{\prime} u_{j}^{\prime}}\right)\right]}_{D_{L, i j} \equiv \text { Molecular Diffusion }} \\
& \underbrace{-\rho\left(\overline{u_{i}^{\prime} u_{k}^{\prime}} \frac{\partial u_{j}}{\partial x_{k}}+\overline{u_{j}^{\prime} u_{k}^{\prime}} \frac{\partial u_{j}}{\partial x_{k}}\right)}_{P_{i j} \equiv \text { Stress Production }}-\underbrace{\rho \beta\left(g_{i} \overline{u_{j}^{\prime} \theta}+g_{j} \overline{u_{i}^{\prime} \theta}\right)}_{G_{i j} \equiv \text { Buoyancy Production }}+\underbrace{p^{\prime}\left(\frac{\partial u_{i}^{\prime}}{\partial x_{j}}+\frac{\partial u_{j}^{\prime}}{\partial x_{i}}\right)}_{\phi_{i j} \equiv \text { Pressure Strain }}-\underbrace{2 \mu \frac{\partial u_{i}^{\prime} \frac{\partial u_{j}^{\prime}}{\partial x_{k}} \frac{\partial x_{k}}{\partial}}{D_{i}}}_{\varepsilon_{i j} \equiv \text { Dissipation }} \\
& \underbrace{-2 \rho \Omega_{k}\left(\overline{u_{j}^{\prime} u_{m}^{\prime}} \varepsilon_{i k m}+\overline{u_{i}^{\prime} u_{m}^{\prime}} \varepsilon_{j k m}\right)}_{F_{i j} \equiv \text { Production by System Rotation }}+\underbrace{S_{\text {user }}}_{\text {User-Defined SourceTerm }}
\end{aligned}
$$

Of the various terms in these exact equations, $C_{i j}, D_{L, i j}, P_{i j}$, and $F_{i j}$ do not require any modeling. However, $D_{T, i j}, G_{i j}, \phi_{i j}$, and $\varepsilon_{i j}$ need to be modeled to close the equations:

- $\quad D_{T, i j}$ is turbulent diffusive transport term and can be approximated by Daly and Harlow's generalized gradient-diffusion hypothesis [80]:

$$
D_{T, i j}=\frac{\partial}{\partial x_{k}}\left(C_{s} \frac{k \overline{u_{k}^{\prime} u_{l}^{\prime}}}{\varepsilon} \frac{\partial \overline{u_{i}^{\prime} u_{j}^{\prime}}}{\partial x_{l}}\right)
$$

This equation can result in numerical instabilities, so it has been simplified as follows:

$$
D_{T, i j}=\frac{\partial}{\partial x_{k}}\left(\frac{\mu_{t}}{\sigma_{k}} \frac{\partial \overline{u_{i}^{\prime} u_{j}^{\prime}}}{\partial x_{k}}\right)
$$

By applying Equation 3-8 to the case of a planar homogeneous shear flow, a value of $\sigma_{k}=0.82$ is derived [81]. $\mu_{t}$ is computed using the following equation:

$$
\mu_{t}=\rho C_{\mu} \frac{k^{2}}{\varepsilon}
$$

and $C_{\mu}=0.09$, which is an empirical constant specified in the turbulence model [76]. 
- The production terms due to buoyancy, $G_{i j}$, are modeled as

$$
G_{i j}=-\frac{\mu_{t}}{\rho \operatorname{Pr}_{t}}\left(g_{i} \frac{\partial \rho}{\partial x_{j}}+g_{j} \frac{\partial \rho}{\partial x_{i}}\right)
$$

where $\operatorname{Pr}_{t}$ is the turbulent Prandtl number, which is a non-dimensional term defined as the ratio between the momentum eddy diffusivity and the heat transfer diffusivity. From experimental data, $\operatorname{Pr}_{\mathrm{t}}$ ranges from 0.7 to 0.9 depending on the Prandtl number of the fluid and has an average value of 0.85 [82] which is used for this equation. So, this value is used for $\operatorname{Pr}_{\mathrm{t}}$ in Equation 3-10 and $g_{i}$ is the component of the gravitational vector in the $i^{\text {th }}$ direction.

- $\phi_{i j}$, which is the pressure strain component can be calculated from:

$$
\begin{aligned}
& \phi_{i j}=-C_{1} \rho \frac{\varepsilon}{k}\left[\overline{u_{i}^{\prime} u_{j}^{\prime}}-\frac{2}{3} \delta_{i j} k\right]-C_{2}\left[\left(P_{i j}+F_{i j}+G_{i j}-C_{i j}\right)-\frac{2}{3} \delta_{i j}(P+G-C)\right] \\
& +C_{1}^{\prime} \frac{\varepsilon}{k}\left(\overline{u_{k}^{\prime} u_{m}^{\prime}} n_{k} n_{m} \delta_{i j}-\frac{3}{2} \overline{u_{i}^{\prime} u_{k}^{\prime}} n_{j} n_{k}-\frac{3}{2} \overline{u_{j}^{\prime} u_{k}^{\prime}} n_{i} n_{k}\right) \frac{k^{3 / 2}}{C_{\ell} \varepsilon d} \\
& +C_{2}^{\prime} \frac{\varepsilon}{k}\left(\phi_{k m, 2} n_{k} n_{m} \delta_{i j}-\frac{3}{2} \phi_{i k, 2} n_{j} n_{k}-\frac{3}{2} \phi_{j k, 2} n_{i} n_{k}\right) \frac{k^{3 / 2}}{C_{\ell} \varepsilon d}
\end{aligned}
$$

where $P_{i j}, F_{i j}, G_{i j}$, and $C_{i j}$ are defined in Equation 3-6 and $P=\frac{1}{2} P_{k k}, G=\frac{1}{2} G_{k k}$, and $C=\frac{1}{2} C_{k k} \cdot C_{\ell}=\frac{C_{\mu}^{3 / 4}}{\kappa}(\kappa=$ the von Kármán constant $=0.4187)$ and $C_{1}=1.8$, $C_{2}=0.6, C_{1}^{\prime}=0.5$, and $C_{2}^{\prime}=0.3$ are model constants [83] and [84]. When the RSM is applied to near-wall flows using the enhanced wall treatment these constants will be different since they become functions of the Reynolds stress invariants and the turbulent Reynolds number.

- The dissipation rate, $\varepsilon_{i j}$, is modeled as: 


$$
\varepsilon_{i j}=\frac{2}{3} \delta_{i j}\left(\rho \varepsilon+\frac{2 \rho \varepsilon k}{a^{2}}\right)
$$

where $a$ is the speed of sound and the scalar dissipation rate, $\varepsilon$, is computed with a model transport equation [75].

Also, it should be mentioned that the presence of walls considerably affects turbulent flows. The reason is that the tangential velocity fluctuations are reduced due to viscous damping and the normal fluctuations are reduced due to kinematic blocking very close to the wall. In addition, the turbulence is rapidly increased by the production of turbulence kinetic energy due to the large gradients in mean velocity. Therefore, consideration needs to be given to make turbulence models suitable for wall bounded flows.

The near-wall region consists of three layers. The closest region to the wall is called the viscous sublayer, where the flow can be considered laminar and viscosity affects momentum and heat or mass transfer. The outer layer which is affected by turbulence is called the fully turbulent layer and the third layer is the interim region which is affected by both viscosity and turbulence (Figure 3-1).

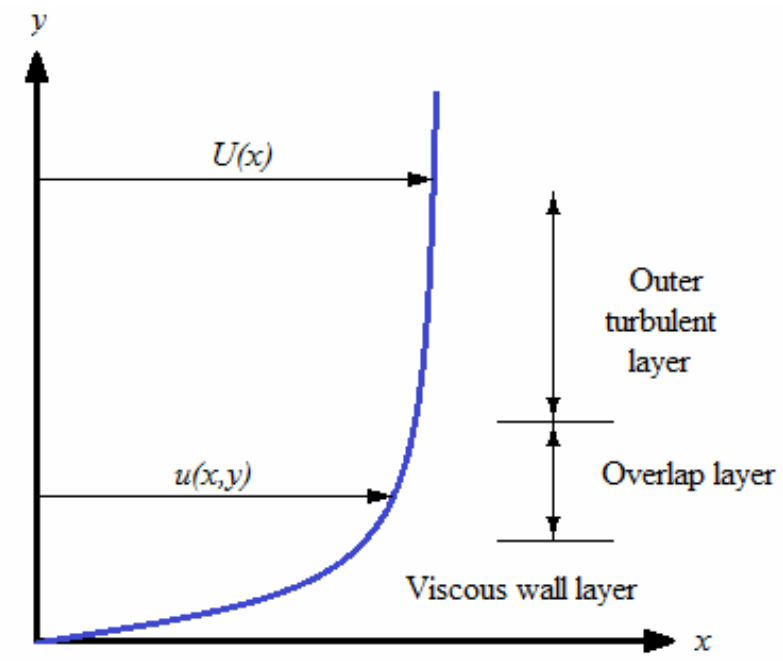

Figure 3-1 Typical velocity distribution in turbulent flow near a wall 
There are two important dimensionless groups which are $u^{+}=\frac{u}{u_{*}}$ and $y^{+}=\frac{\rho u_{*} y}{\mu}$, where $u_{*}$ is friction velocity and equal to $\sqrt{\frac{\tau_{w}}{\rho}}$ [83]. In the viscous sublayer, these two quantities have a linear relationship:

$$
u^{+}=y^{+}
$$

while, for the outer layer $\left(y^{+}>30\right)$ the velocity variation with $y$ can be fit by a logarithmic expression:

$$
u^{+}=\frac{1}{\kappa} \ln \left(y^{+}\right)+B
$$

where $\kappa=0.4187$ and $B$ is a constant and different values for it can be found from the literature [83]-[87]. This equation is valid for all turbulent flows past smooth walls at high Reynolds number. Figure 3-2 shows the close agreement between experimental data and semi-empirical equations 3-13 and 3-14.

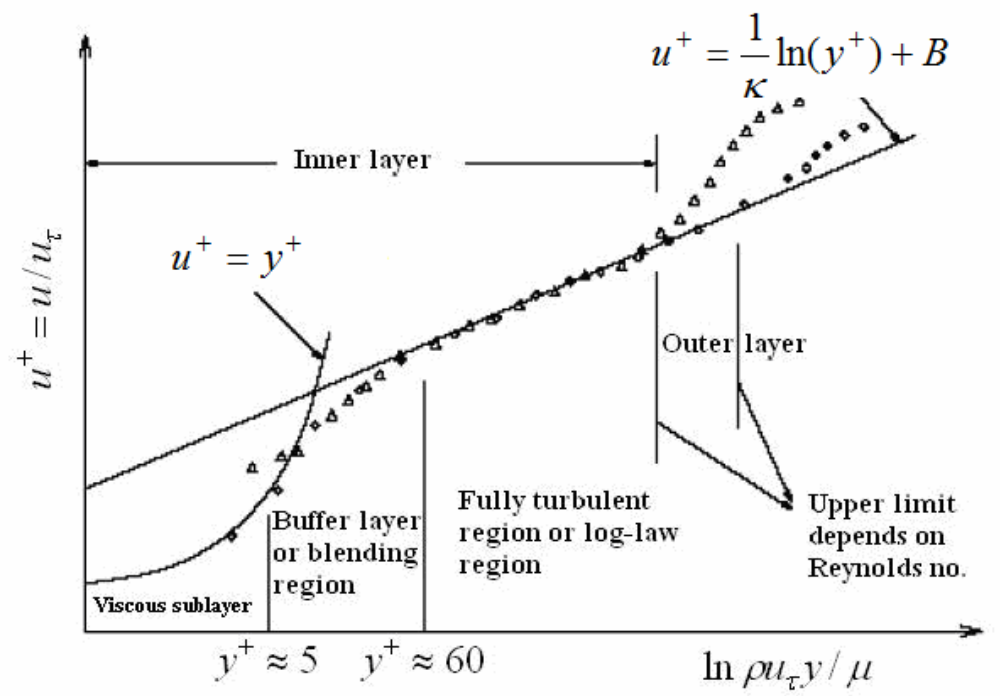

Figure 3-2 The turbulent boundary layer (adapted from ref. [75])

There are two methods to model the near-wall region. In the first approach, turbulence models are modified to be able to resolve the viscosity affected region with a very fine 
mesh near the walls, which is called near-wall modeling. In the other approach the nearwall region is not resolved and semi-empirical formulae are used to bridge over the viscous sub-layer, between the wall and the fully-turbulent region (Figure 3-3). This approach is called wall function modeling. The wall function approach, especially for high Reynolds number flows, is a practical option because it not only saves the computational cost, but also is robust and maintains the physical properties of the viscous sub-layer through the empirical function [75]. There are three different wall function approaches:

- Standard wall functions

- Non-equilibrium wall functions

- Enhanced wall treatment

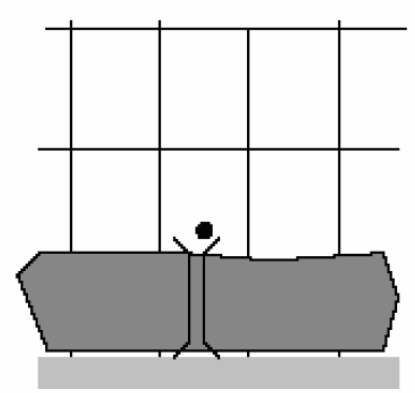

Wall Function Approach

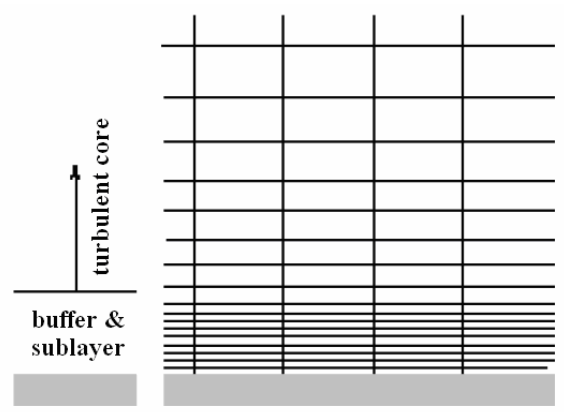

Near-Wall Model Approach

Figure 3-3 Near-wall treatments (adapted from ref. [75])

The standard wall functions work well for many cases, unless the flow situations are too different from the derivation assumptions, such as the local equilibrium hypotheses, which means the production of $k$ and its dissipation rate are assumed to be equal in the wall-adjacent control volume and the first grid point should be within the logarithmic region (Figure 3-2), i.e. when $y^{+}>30$ [76]. Therefore, in the case of flows with high pressure gradients and non-equilibrium conditions this model is not so useful. 
For complex flow phenomena such as separation, reattachment, and impingement, where high pressure gradients occur, the two-layer-based, non-equilibrium wall functions are recommended [88]. In such flows, improvements can be obtained, especially in the prediction of wall shear (skin friction coefficient). Considering the dominance of the above-mentioned flow phenomena in the flow in the elliptical cavity, the two-layer-based non-equilibrium wall functions are the most adequate wall treatment approach for simulation of flow in the cavity. Comparison of the results of the simulations involving this approach with previously published experimental results, which is presented in section 3.7, confirms that this approach is appropriate for the flow under investigation in the present study.

Sometimes, the flow conditions are greatly different from the ideal condition assumptions which are used for these two models (such as severe pressure gradients leading to boundary layer separations and high three-dimensionality in the near-wall region, as in the case as Ekman spiral flow and strongly skewed boundary layers). In these cases, the near-wall modeling approach should be combined with adequate mesh resolution and enhanced wall treatment.

Enhanced wall treatment is a combination of the two-layer model with enhanced wall functions. If the near-wall mesh is fine enough $\left(y^{+} \approx 1\right)$, then the enhanced wall treatment will be identical to the traditional two-layer zonal model. This model is suitable for cases where a combination of mesh sizes, including coarse meshes (wall function meshes), fine meshes (low Reynolds number meshes), and intermediate meshes exists near the walls. As a result of using this approach, the accuracy of the standard two-layer approach for fine near-wall meshes can be achieved [89]. This approach may also be adequate for simulation of flow in the elliptical cavity. However, the combination of the mesh size requirements in this approach, especially in the case of the complex, threedimensional geometry of the cavity, leads to a prohibitively large grid, which would not be practical for the number of simulations required for multiple combinations of cavity depth and yaw angle. 
Ideally, a direct numerical simulation to resolve all turbulence scales is the best approach to any problem, from the point of view of accuracy. However, such a simulation becomes too costly, when complex geometries and relatively large amounts of turbulence are involved. It can be shown that the number of grid points in each direction is proportional to $\operatorname{Re}_{L}^{3 / 4}$, where $\operatorname{Re}_{L}$ is the Reynolds number based on the magnitude of the velocity fluctuations and the integral scale [90]. Therefore, for a three-dimensional unsteady flow, the cost of a simulation typically scales as $\operatorname{Re}_{L}^{3}$. For this reason, DNS is possible only at low Reynolds numbers and this model is not practical for elliptical cavity flow simulation, hence the RANS method and Reynolds Stress Model (RSM) are used. In the next sections, the details of the computational domain and grid, boundary conditions, and the solution parameters will be discussed.

\subsection{Computational Domain}

The solution domain is bounded by four solid walls representing the wind tunnel test section, 11.4D in width and 4D in height, where $\mathrm{D}$ is the length of the minor axis of the cavity $(53.75 \mathrm{~mm})$. These dimensions are equivalent to the experiment of Hering and Savory which were conducted in the Boundary Layer Wind Tunnel Laboratory (BLWTL) at the University of Western Ontario [6].

The wind tunnel length is $6.5 \mathrm{~m}(120.8 \mathrm{D})$ and the cavity model was placed a distance of $3.45 \mathrm{~m}$ downstream of the tunnel working section entrance for a thick, fully-developed boundary layer to develop. In order to reduce the number of cells and have a higher resolution inside and around the cavity, the streamwise length is not completely modeled. In addition, to have the same boundary layer condition, the fully-developed experimental profiles are defined at the inlet which will be explained in detail in section 3.5. The domain extended 5D upstream and 15D downstream as shown in Figure 3-4.

The geometric model consists of an elliptical cavity located inside the test section. The cavity has a 2:1 ratio of major axis to minor axis length. The modeled cavity depth to the minor axis length ratios (h/D) are 0.1, 0.2, 0.35, 0.5, 0.7, and 1.0. Also, the yaw angles 
are $0,15,30,45,60$, and 90 degrees, where $0^{\circ}$ represents the minor axis parallel to the boundary layer flow direction.

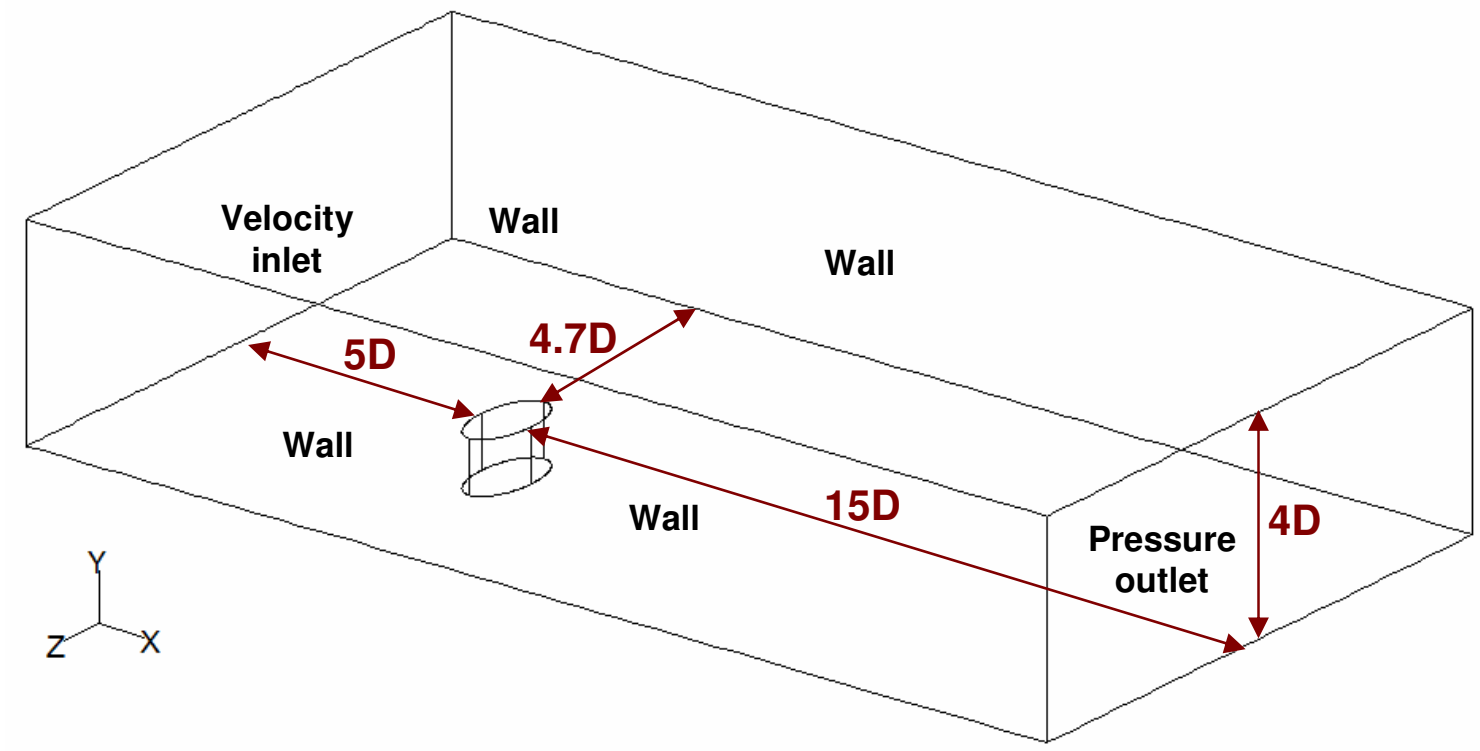

Figure 3-4 Geometry and boundary types

\subsection{Computational Grid}

A structured mesh consisting of hexahedral shaped cells has been used for discretization of the solution domain. In order to have a better control on the mesh size in important areas, the computational domain is divided to several sub-volumes. The resulting solution grid is shown in Figure 3-5. A similar grid structure is used for all cavity depths and the only difference among the grids for the different cavity depths are the cell aspect ratios in the interior of the cavity.

The choice of grid may influence the accuracy and convergence of a solution. Although a finer mesh usually gives a more accurate solution, it increases the computational cost. Therefore, an optimum number of cells should be chosen to achieve accurate results within a reasonable computational cost. In the meantime, the grid should be fine enough so that the results can be considered independent of the mesh size. 


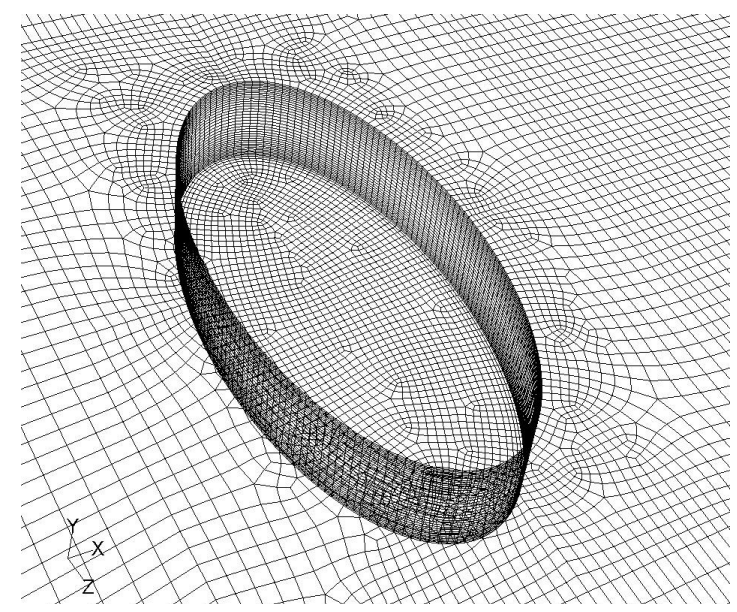

Figure 3-5 Computational grid in the cavity and the surroundings

Independence of the numerical results from the grid size was checked by comparing the pressure coefficient on the centreline of the cavity base, and also the profiles of $\bar{u} / U_{\text {inf }}$ and $\overline{u^{\prime} u^{\prime}} / U_{\text {inf }}^{2}$ on a vertical line located at the centre of the cavity, based on solutions with various numbers of cells. The grid independence criterion was considered to be a less than $2.5 \%$ variation in above mentioned profiles as a result of the change in grid size. Multiple simulations were carried out, in which the number of cells was increased in 150,000 cell increments. The results of this comparison are presented in Table 3-1. Since the changes in the selected parameters were less than the $2.5 \%$ criterion for grids finer than 800,000 cells, this number of cells was chosen for the numerical simulations. The centreline $C_{p}, \bar{u} / U_{\text {inf }}$, and $\overline{u^{\prime} u^{\prime}} / U_{\text {inf }}^{2}$ profiles are shown in Figure 3-6.

Table 3-1 Variations of selected parameters with the number of cells

\begin{tabular}{|l|c|c|}
\hline \multirow{2}{*}{ Parameter } & \multicolumn{2}{|c|}{ Average absoloute variation } \\
\cline { 2 - 3 } & 650,000 to 800,000 cells & 800,000 to 950,000 cells \\
\hline$C_{p}$ & $8.4 \%$ & $2.3 \%$ \\
\hline $\bar{u} / U_{\text {inf }}$ & $1.5 \%$ & $1.0 \%$ \\
\hline$\overline{u^{\prime} u^{\prime}} / U_{\text {inf }}^{2}$ & $3.3 \%$ & $1.3 \%$ \\
\hline
\end{tabular}




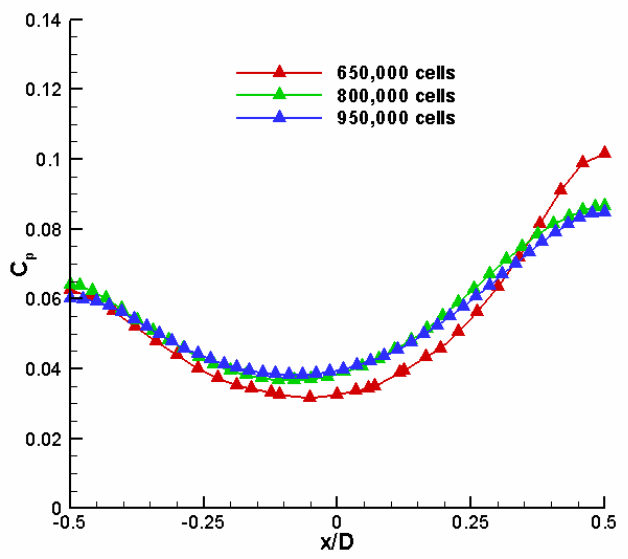

(a)

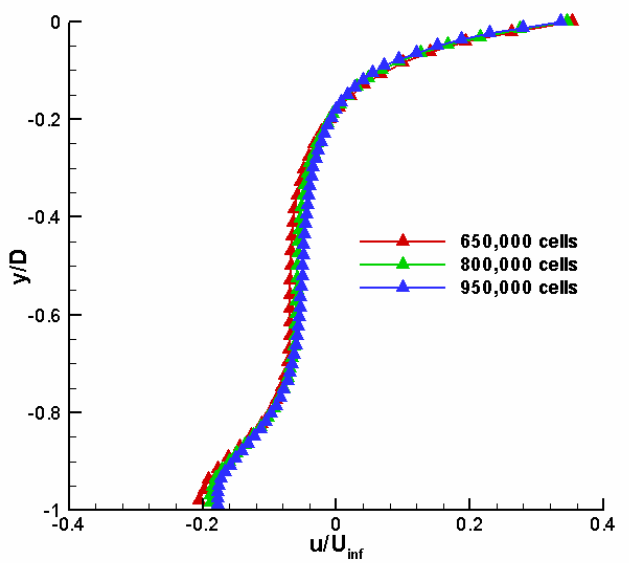

(b)

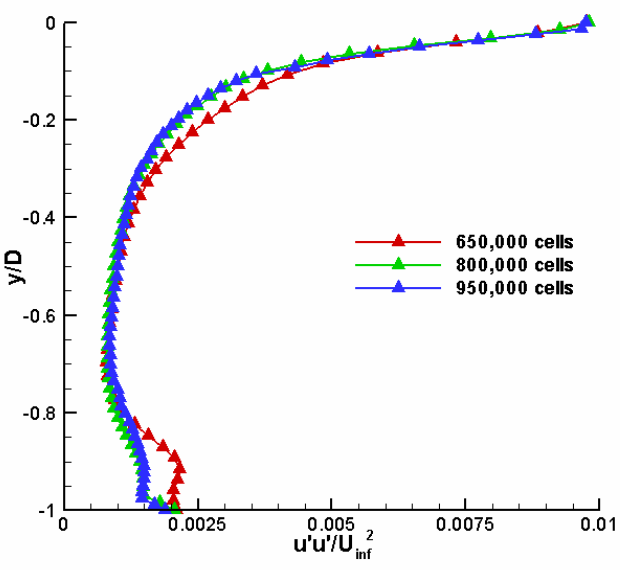

(c)

Figure 3-6 Comparison of $C_{p}(\mathbf{a}), \bar{u} / U_{\mathrm{inf}}(\mathbf{b})$, and $\overline{u^{\prime} u^{\prime}} / U_{\mathrm{inf}}^{2}(\mathbf{c})$ for three different numbers of cells

\subsection{Boundary Conditions}

The solution domain is defined by the following boundaries, as shown in Figure 3-4:

- Four solid walls representing the wind tunnel test section (as described in the section 3.3). All walls, including the wind tunnel test section and cavity body, are stationary with the no-slip condition.

- A velocity inlet point profile based on the actual boundary layer wind tunnel data of Hering and Savory [6] was used at the velocity inlet boundary. The boundary layer parameters are mentioned in detail in summary section of the next chapter. 
The following power law curve was fit to the experimental data and was used to generate the point profile data for the inlet boundary (Figure 3-7):

$$
\frac{\bar{u}}{U_{\mathrm{inf}}}=\left(\frac{y}{\delta}\right)^{1 / 6.9}
$$

in which $U_{\text {inf }}$ is the free stream velocity $(27 \mathrm{~m} / \mathrm{s})$, and $\delta$ is the estimated boundary layer thickness $(59 \mathrm{~mm})$. This free stream velocity results in a Reynolds number of $9.1 \times 10^{4}$, based on the minor axis (D) of the cavity. This curve is similar to the typical $1 / 7^{\text {th }}$ power law for a smooth wall turbulent boundary layer [83]. The details of the boundary layer, including boundary layer thickness $(\delta)$, displacement thickness $\left(\delta^{*}\right)$, momentum thickness $(\theta)$, shape factor $(\mathrm{H})$, skin friction $\left(\mathrm{C}_{\mathrm{f}}\right)$, and friction velocity $\left(\mathrm{u}_{*}\right)$, are presented in the summary section of Chapter 4.

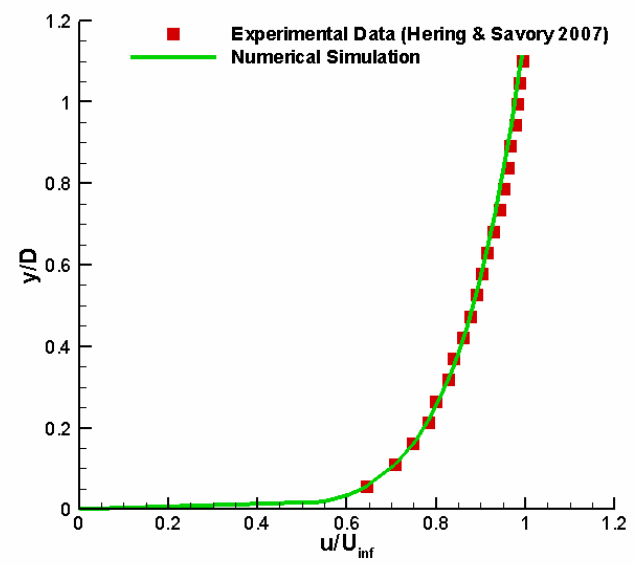

Figure 3-7 Boundary layer velocity profile at the inlet

- In order to have similar turbulent component profiles to the measurements in the experiment, point profiles for Reynolds stress components, turbulence kinetic energy $(k)$, and dissipation rate $(\varepsilon)$ are used at the inlet.

For the Reynolds stress components, the experimental values measured by Hering [6] were used (Figure 3-8). While these measurements were for the half of the tunnel height, these profiles are mirrored to cover the whole height. It is assumed 
that the Reynold stress components in the $y$ and $z$ directions are similar, therefore: $\overline{w^{\prime} w^{\prime}}=\overline{v^{\prime} v^{\prime}}$. Also, as these stresses arise due to shear and there is only shear in $\mathrm{y}$ direction through the boundary layer, it can be assumed that $\overline{u^{\prime} w^{\prime}}=\overline{v^{\prime} w^{\prime}}=0$.

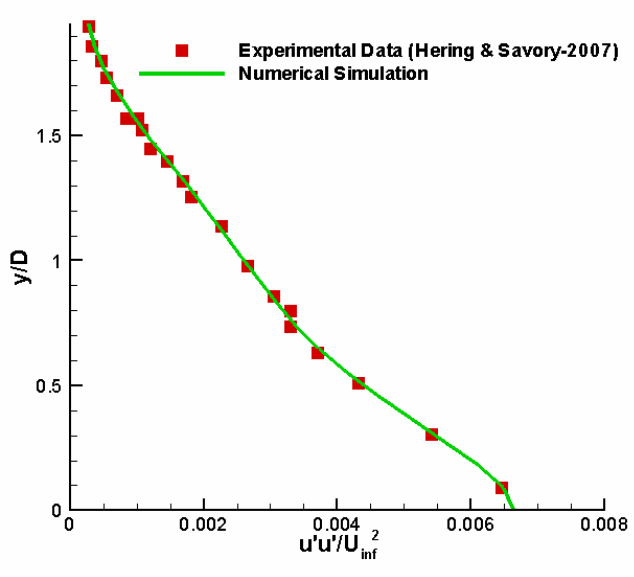

(a)

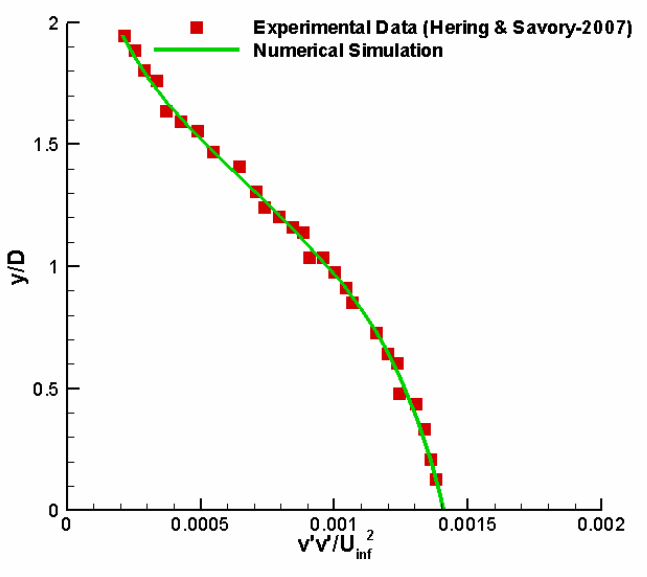

(b)

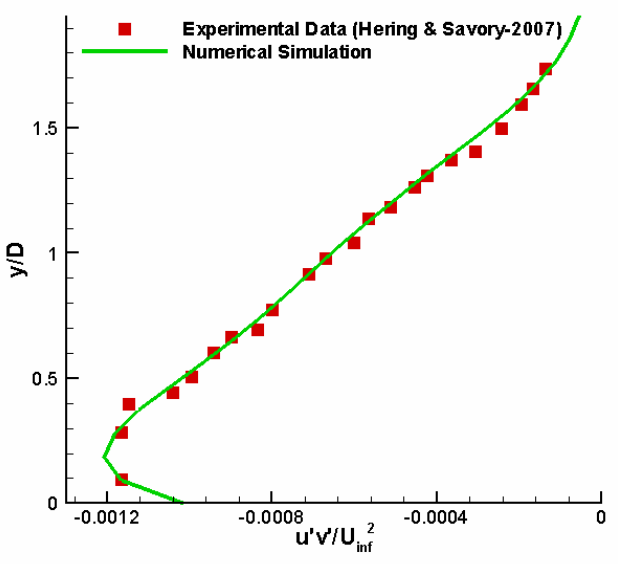

(c)

Figure 3-8 Dimensionless $\overline{u^{\prime} u^{\prime}}$ (a), $\overline{v^{\prime} v^{\prime}}$ (b), and $\overline{u^{\prime} v^{\prime}}$ (c) Reynolds stress profiles at the inlet

For the turbulence kinetic energy $(k)$ inlet profile, Equation 3-5 is used and the dissipation rate $(\varepsilon)$ is determined from the following relationship [91]:

$$
\varepsilon=C_{\mu}^{3 / 4} \frac{k^{3 / 2}}{l}
$$


where $C_{\mu}=0.09$, and $l$ is the turbulence length scale. For wall-bounded flows in which the inlets involve a turbulent boundary layer, the recommended value for length scale is $l=0.4 \delta$, in which $\delta$ is the thickness of the boundary layer [75]. In the experiments by Hering [58], the thickness of the boundary layer upstream of the cavity is $0.06 \mathrm{~m}$, which results in a value of $0.024 \mathrm{~m}$ for the turbulence length scale. The inlet profiles for $k$ and $\varepsilon$ are shown in Figure 3-9.

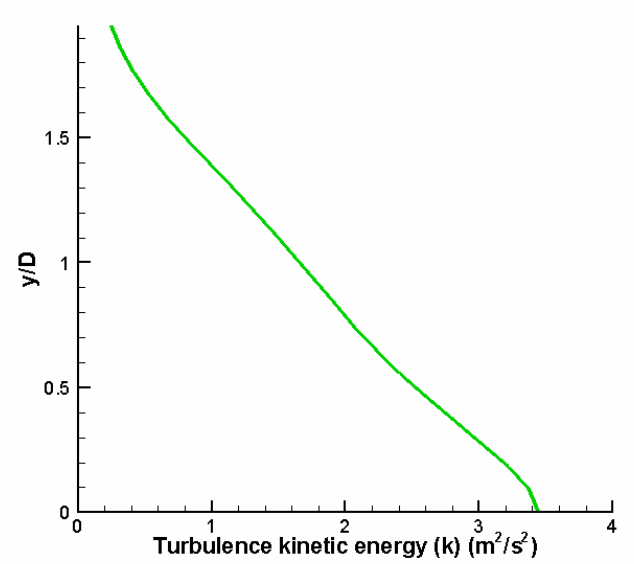

(a)

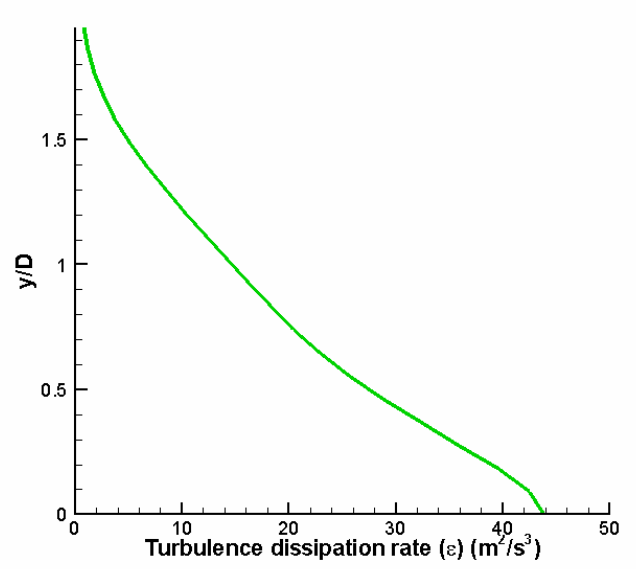

(b)

Figure 3-9 Turbulence kinetic energy $(k)$ (a) and Turbulence dissipation rate $(\varepsilon)(b)$ at the inlet

- A pressure outlet boundary condition was defined for the wind tunnel outlet. This boundary condition allows the static pressure at outlet to be defined and also if backflow occurs during iteration, pressure outlet boundary condition results in a better rate of convergence [75].

\subsection{Solution Parameters}

The governing equations, which were described in section 3.2, are numerically solved using the steady state three-dimensional segregated implicit solver available in FLUENT 6.3. As was discussed previously in section 2.9, some researchers ([50] and [71]-[73]) have previously shown (taking into consideration the cost, time of computation and required computational resources) that although a steady state solver cannot capture the 
transient terms and fluctuations, it is still able to predict the principal flow phenomena and the mean flow characteristics.

Due to the shear layer separation at the leading edge of the cavity and the non-isotropic turbulence in the experiments, a turbulence model capable of capturing shear layer separation and individual Reynolds stress components is required. Hence the 7-equation Reynolds Stress Model (RSM) is chosen.

As mentioned before, considering the flow behaviour inside the cavity, non-equilibrium wall functions were the most suitable wall function and they were chosen for these numerical simulations.

Reduction of the global residual of the solution of all the governing equations to the order of $10^{-6}$ was selected as the convergence criterion and the discretisation scheme was second order upwind.

For the present work different numerical models, including different grids, domain sizes (upstream and downstream), turbulence intensities, inlet profiles and wall functions and Reynolds Stress Model parameters, were tested and the best numerical model was chosen.

\subsection{Validation of the Results of Numerical Simulations}

In other to validate the numerical results, $\mathrm{C}_{\mathrm{p}}$ contours on cavity walls and around the cavity, and quantitative values of $\mathrm{C}_{\mathrm{p}}$ on the centreline of the cavity base, were compared to Hering's experimental data [58]. This comparison has been conducted for different combinations of yaw angle and cavity depth, Here, the results of this comparison for selected cases will be presented. The results for several other combinations can be found in Chapter 5.

Figure 3-10 shows the comparison of experimental measurements and numerical simulation results for $C_{p}$ profiles on the centreline of the cavity base. The results are presented for three different cavity depths, which include deep cavity $(\mathrm{h} / \mathrm{D}=1.0)$, intermediate-depth cavity $(\mathrm{h} / \mathrm{D}=0.5)$, and shallow cavity $(\mathrm{h} / \mathrm{D}=0.1)$, at two different 
yaw angles of $0^{\circ}$ and $90^{\circ}$. The profiles based on experimental and numerical results follow similar trends for all cases, and the values of $\mathrm{C}_{\mathrm{p}}$ at most of the points are close.

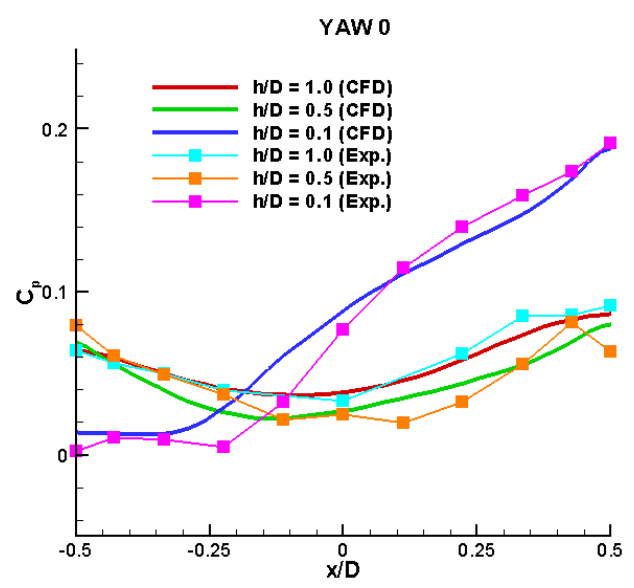

(a)

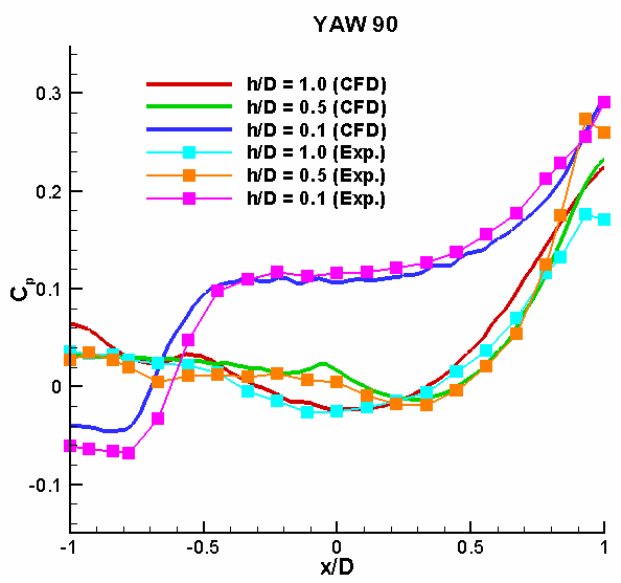

(b)

Figure 3-10 Comparison of $C_{p}$ profiles on the centreline of the cavity base for three different $h / D$ ratios at yaw angles of $0^{\circ}(a)$ and $90^{\circ}(b)$

The quantitative comparison of these profiles is presented in Table 3-2. The results indicate a maximum of $5 \%$ average difference between numerical simulations and experimental data for $\mathrm{C}_{\mathrm{p}}$, which can be considered as a reasonable agreement between the results.

Table 3-2 Quantitative comparison of $C_{p}$ values obtained by numerical simulations and experimental data

\begin{tabular}{|c|c|c|c|c|c|c|}
\hline Yaw angle & \multicolumn{3}{|c|}{$0^{\circ}$} & \multicolumn{3}{c|}{$90^{\circ}$} \\
\hline $\mathrm{h} / \mathrm{D}$ & 0.1 & 0.5 & 1.0 & 0.1 & 0.5 & 1.0 \\
\hline $\begin{array}{c}\text { Average absoloute difference } \\
\text { between numerical simulation and } \\
\text { experimental results for } \mathrm{C}_{\mathrm{p}}(\%)\end{array}$ & 4.9 & 3.8 & 1.9 & 4.0 & 3.2 & 3.8 \\
\hline
\end{tabular}

As an overall representation of numerical and experimental results in comparison, contours of $C_{p}$ on cavity side walls and the area around the cavity for $h / D=1.0$ at $0^{\circ}$ yaw 
angle are shown in Figure 3-11. This figure indicates that the contour shapes and levels are similar for both methods. A more detailed quantitative and qualitative comparative evaluation of the experimental and numerical results, which includes velocity profiles, Reynolds stress profiles, and vortex core position inside the cavity, will be presented in Chapter 5.

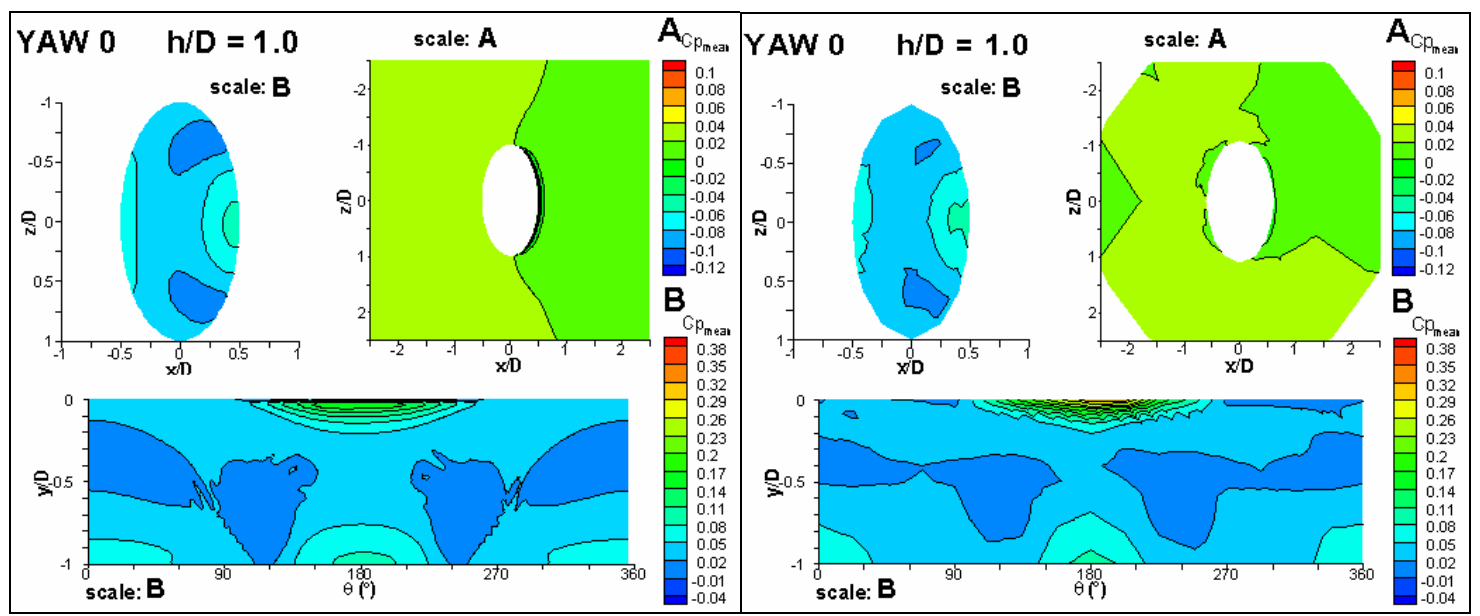

(a)

(b)

Figure 3-11 $C_{p}$ contours on cavity walls and around the cavity for $h / D=1.0$ at $0^{\circ}$ yaw angle based on numerical simulation (a) and experimental results [58] (b)

\subsection{Summary}

In this chapter, details of the numerical simulation settings and procedure were presented. The governing equations and treatment of turbulence in the Reynolds-Averaged NavierStokes (RANS) approach were explained, followed by detailed discussion of near-wall treatment approaches relevant to the cavity flow problem. The set-up and discretization of the computational domain based on the considerations and restrictions imposed by the numerical model were explained. The main specifications of the numerical simulations are as follow:

- The grid was generated using Gambit. The exact dimensions of the wind tunnel test section and the model of the elliptical cavity with an aspect ratio of 2:1 was generated and proper boundary conditions were chosen. 
- Grid independence was checked by comparison of pressure coefficients, velocities, and shear stress components.

- The flow inside and around the cavity was simulated using FLUENT

- The segregated implicit solver was used for three-dimensional steady state simulations. $2^{\text {nd }}$ order spatial discretization was applied.

- Reynolds Stress Model and non-equilibrium wall functions were used for turbulence treatment.

- At the inlet, the velocity profile, turbulent kinetic energy, dissipation rate, and Reynolds stress components were matched to the conditions of the wind tunnel experiments reported in [6] and [58]. The details of these profiles are presented in the summary section of Chapter 4.

- Different combinations of cavity depth and yaw angle, including $\mathrm{h} / \mathrm{D}=0.1$, $0.2,0.35,0.5,0.7$, and 1.0 at yaw angles of $0,15,30,45,60$, and 90 degrees were simulated.

- The results of the numerical simulations were validated through quantitative and qualitative comparison with the experimental data by Hering [58]. These comparisons showed a good qualitative agreement, with a maximum average difference of $5 \%$ in $\mathrm{C}_{\mathrm{p}}$ values, between two sets of data.

More results of the numerical simulations and additional quantitative and qualitative comparison of the data will be presented in Chapter 5 . 


\section{Experimental Methodology}

\subsection{Introduction}

This chapter describes the experimental facilities and measurement equipment and methods, as well as experimental setup, used to obtain the data presented in the next chapter. Also, the uncertainties of these methods and equipment are presented. The model components, design considerations, and manufacturing challenges to obtain the proper boundary layer profile and the best optical quality are explained. The main constraint in designing the model dimensions and boundary layer thickness was to generate the experimental conditions as close as possible to those of Hering [58] in order to obtain comparable results.

\subsection{Wind Tunnel}

The wind tunnel used for the present experiments was a suction type open loop tunnel in the Boundary Layer Wind Tunnel Lab (BLWTL) of the University of Western Ontario. The test section dimensions were $0.45 \mathrm{~m} \times 0.46 \mathrm{~m} \times 1.5 \mathrm{~m}$ with an adjustable roof. A schematic representation of the wind tunnel is shown in Figure 4-1. There were a honeycomb and a fine grid mesh at the inlet of the tunnel to straighten the incoming flow and reduce free-stream turbulence. After the diffuser, there was a 9-blade, 0.8m diameter fan which was powered by a hydraulic motor. An almost uniform free stream was shown across the test section using hot wire anemometry within the $1 \%$ measurement uncertainty in previous experiments [92]. The maximum sustainable free stream speed of the tunnel was $20 \mathrm{~m} / \mathrm{s}$ and the turbulence intensity was $2 \%$.

It should be mentioned that this tunnel was not the same wind tunnel which was used by Hering [58], due to the limited optical access to the test section of that tunnel. The lower surface of the test section of the current tunnel is made of glass, as well as two side windows. Therefore, the optical access through two transparent perpendicular surfaces, which is an essential condition for particle image velocimetry (PIV) measurements, was made possible by this tunnel. 


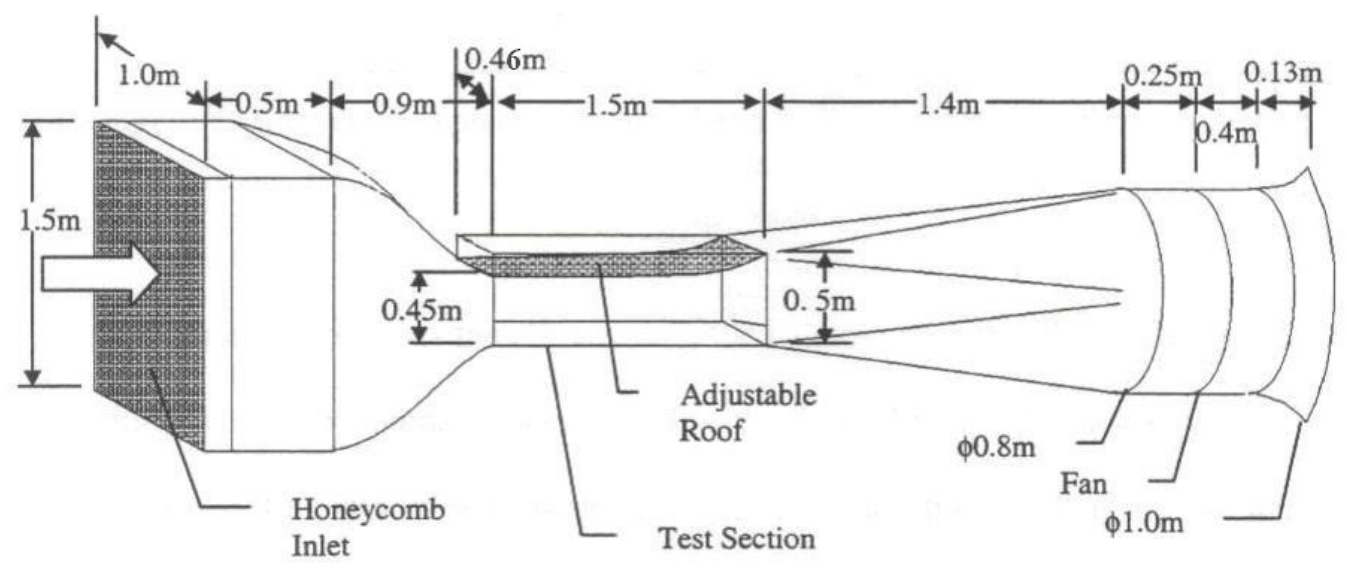

Figure 4-1 Schematic representation of the wind tunnel [92]

\subsection{Model Specifications}

The model consisted of a circular ground plate at the centre of which the cavity is located, a base plate, a trailing edge flap, and eight support legs (Figure 4-2). All model components were made of acrylic due to its ease of manufacturing to obtain the required transparency for PIV measurements.

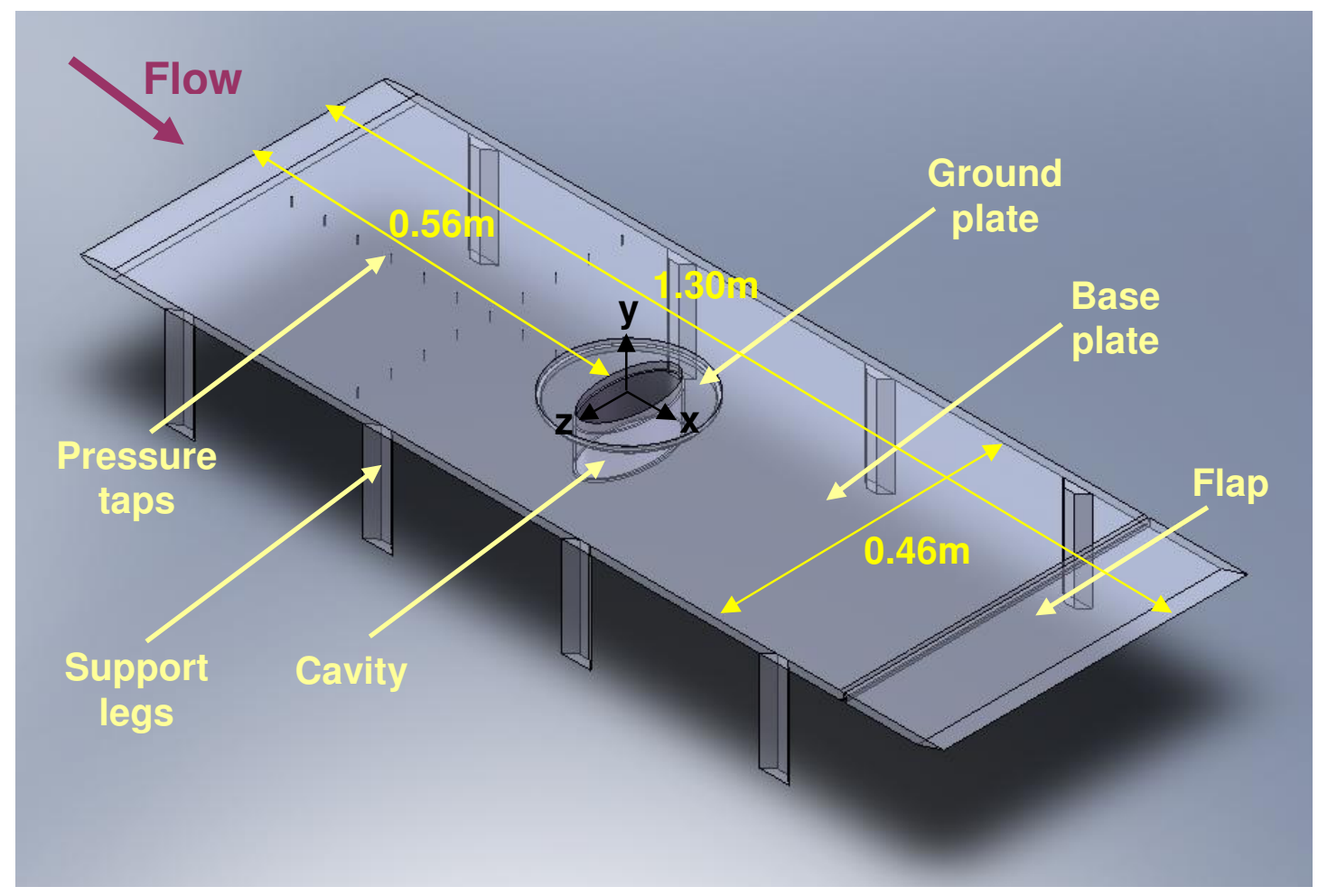

Figure 4-2 Schematic representation of the model 
The dimensions and design considerations of each of these components are as follows (all drawings can be found in Appendix A):

\section{- Ground plate and cavity}

The ground plate was a circular shaped plate with a 2:1 aspect ratio elliptical cavity in the centre. The plane diameter was $20 \mathrm{~cm}$ and the cavity minor axis and its depth were both $7.25 \mathrm{~cm}$. The cavity depth could be changed by stacking elliptical solid cylinders made of acrylic, with various heights inside the cavity. The reason this cavity size was chosen is to obtain the same Reynolds number, based on the minor axis (D) of the cavity $\left(9.1 \times 10^{4}\right)$ as the experiments of Hering [58] at the maximum velocity of the tunnel $(20 \mathrm{~m} / \mathrm{s})$. Also, these cavity dimensions allowed a distance of $19 \mathrm{~cm}(2.6 \mathrm{D})$ from the test section side walls to reduce the wall effect on the cavity flow. The ground plate had a step-shaped circumference, which could be flush-mounted inside the base plate while the cavity yaw angle could be adjusted to $0,15,30,45,60$, and 90 degrees (Figure 4-3).

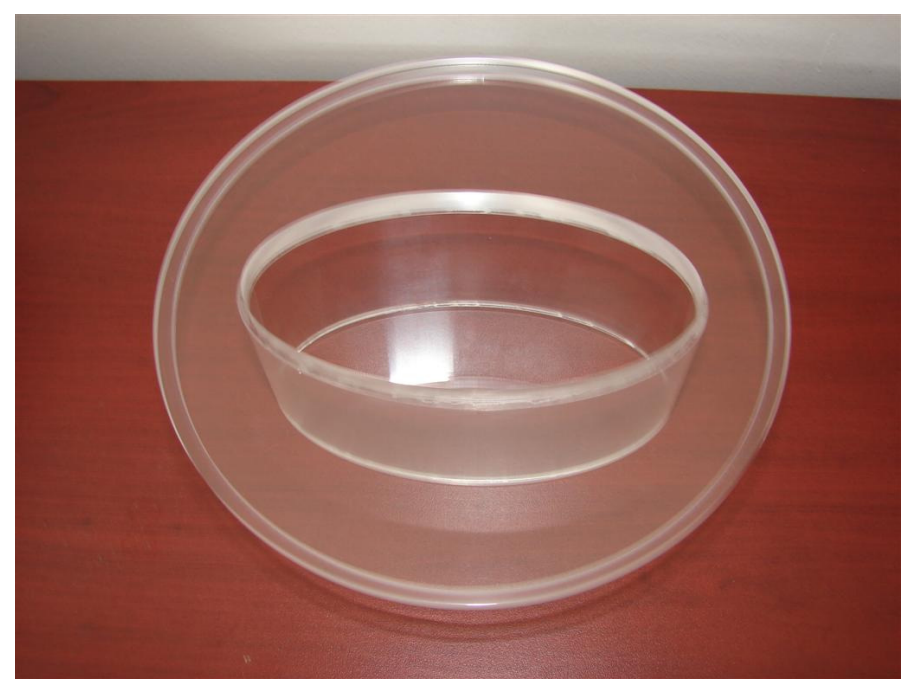

Figure 4-3 Cavity model and ground plate

For changing the cavity depth two different elliptical solid cylinders were used. The elliptical section of the cylinders was made slightly smaller than the cavity to make it possible for them to be slid inside the cavity and at the same time fit in the cavity 
properly without any noticeable gap. The height of the cylinders were $2.18 \mathrm{~cm}$ and $3.63 \mathrm{~cm}$ to obtain $\mathrm{h} / \mathrm{D}=0.7$ and 0.5 respectively. Those cylinders were completely transparent to provide the optimum optical access for the measurements. Due to the optical blockage of the side view of the cavity by the ground and base plates it was not possible to do the PIV measurements for shallower cavities.

\section{- Base plate}

The base plate was a flat plate with a semi-elliptical shaped leading edge. The aspect ratio was $8: 1$ and the chord of the elliptical section was $5 \mathrm{~cm}$. This configuration was chosen to prevent flow separation at the leading edge [93]. The plate dimensions were $116 \mathrm{~cm} \times 46 \mathrm{~cm} \times 1.27 \mathrm{~cm}$ (including leading edge). The leading edge upper surface was covered with a 50D grit garnet paper strip (Figure 4-4). This surface roughness forces the boundary layer to transition to turbulence [94]. To check the flow attachment on the surface for each yaw angle, which leads to a different blockage ratio on the bottom side of the plate, the pressure distribution was measured in the streamwise and spanwise directions using nine and seven pressure taps (brass tubing), respectively. The pressure taps had an inner diameter of $0.8 \mathrm{~mm}$ and an outer diameter of $1.2 \mathrm{~mm}$ and the spacing between them was $50 \mathrm{~mm}$.

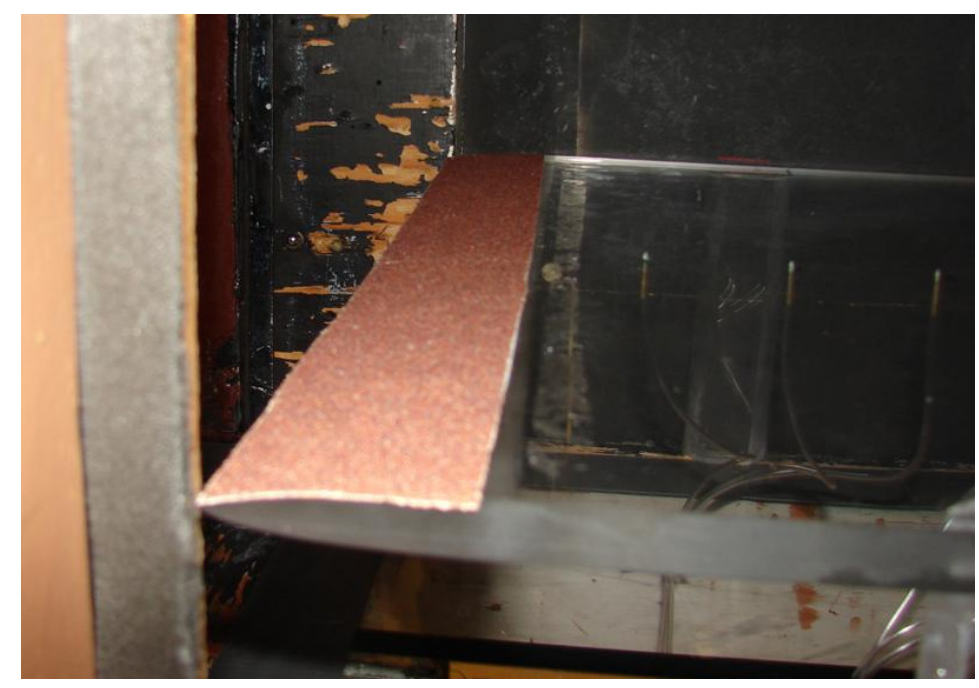

Figure 4-4 Leading edge shape and surface roughness 
At the centre of the plate, $0.5 \mathrm{~m}$ downstream, there was a circular opening with a stepshaped circumference, into which the ground plate is fitted. The reason this opening was located at the centre of the base plate was that a proper distance upstream the cavity was needed to obtain a developed thick boundary layer profile and also the cavity should be at a distance from the trailing edge so that the wake of the cavity was not affected by the trailing edge proximity.

\section{- Support legs}

The base plate was positioned on four pairs of support legs (Figure 4-5). This number of legs was chosen to minimize the base plate deflection due to its weight. The following formula gives the maximum deflection at the centre of a rectangular plate simply supported at its four corners [95]:

$$
[w]_{0,0}=\frac{q a^{4}}{4 E h^{3}}\left(\left(1+\mu-\mu^{2}\right)\left(1+\frac{b^{4}}{a^{4}}\right)-2(7 \mu-1)\left(\frac{b^{2}}{a^{2}}\right)\right)
$$

where, $[w]_{0,0}$ is the maximum deflection at the centre of the plate, $b$ and $a$ are half of the width and the length of the plate respectively, $E$ is the modulus of elasticity, $h$ is the plate thickness, $q$ is the intensity of uniformly distributed load, and $\mu$ is Poisson's ratio.

Considering the spacing of $0.4 \mathrm{~m}$ between each two sets of legs, the maximum deflection at the centre of the plate due to self weight is $0.2 \mathrm{~mm}$. It should be mentioned that this equation is for a simply supported plate, therefore it overestimates the deflection since the support legs of the model are glued to the base plate. The first pair of legs was located $0.15 \mathrm{~m}$ downstream from the leading edge, therefore they did not interfere with the flow and their cross-section was chosen in a way to reduce the flow separation and wake effects with $40^{\circ}$ chamfered edge in front and aft. The height of the legs was $0.15 \mathrm{~m}$ which allowed the cavity to be mounted inside the base plate and also a $0.3 \mathrm{~m}$ (4D) distance from the cavity lip to the test section roof minimized any flow interference due to the wall effects. 


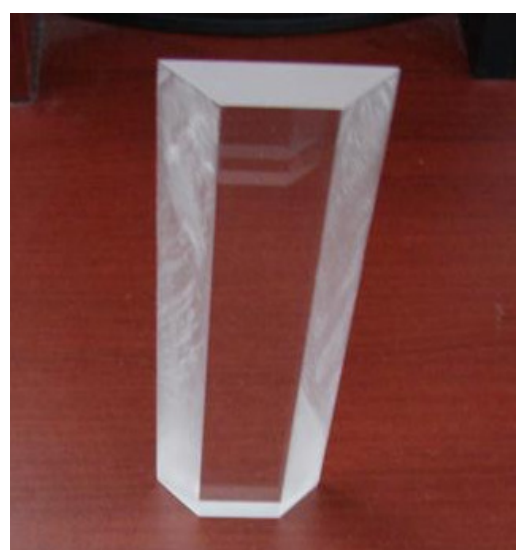

Figure 4-5 Support leg

\section{- Trailing edge flap}

Due to the presence of the cavity model and the resultant blockage and also the location of the base plate which was closer to the ground than the roof, flow circulated around the plate. In order to get a well-attached flow on the upper surface of the base plate, a flap with a sharp trailing edge was used at the downstream end, which could be raised to balance the blockage between two sides of the plate and control the attachment line on the leading edge [96]. The flap chord ratio compared to the plate length varied between 9.6\%-12.0\% in similar experiments [94]-[98]. The flap chord was $15 \mathrm{~cm}$ which was a chord ratio of $11.5 \%$. The flap was attached to the base plate with two hinges at the two ends (Figure 4-6).

To achieve the same attached flow for all cavity yaw angles (different blockages), a series of experiments were performed and a range of flap angles were tested for each yaw angle. In each combination of yaw angle and flap angles, the streamwise and spanwise pressure distributions were measured. To obtain the same plate pressure distribution upstream of the cavity, the flap angle varied between $7^{\circ}-15^{\circ}$ for the smallest (yaw angle $=90^{\circ}$ ) and largest blockage (yaw angle $=0^{\circ}$ ), respectively. Figure 4-7 illustrates the $C_{P}$ distribution in both directions for the final combination of yaw and flap angles $(\alpha)$. Mean pressure coefficient $\left(\mathrm{C}_{\mathrm{P}}\right)$ values were calculated by applying the following equation: 


$$
C_{p}=\frac{\bar{P}-P_{\text {static }}}{P_{d y n a m i c}}
$$

where $\bar{P}$ is the mean pressure at the point for which the pressure coefficient is being calculated. Details of the pressure measurement process and the location of $P_{\text {static }}$ measurements are explained in section 4.5. It should be mentioned that in part (a) of this figure $x^{\prime} / \mathrm{D}$ shows the streamwise distance from the plate leading edge.

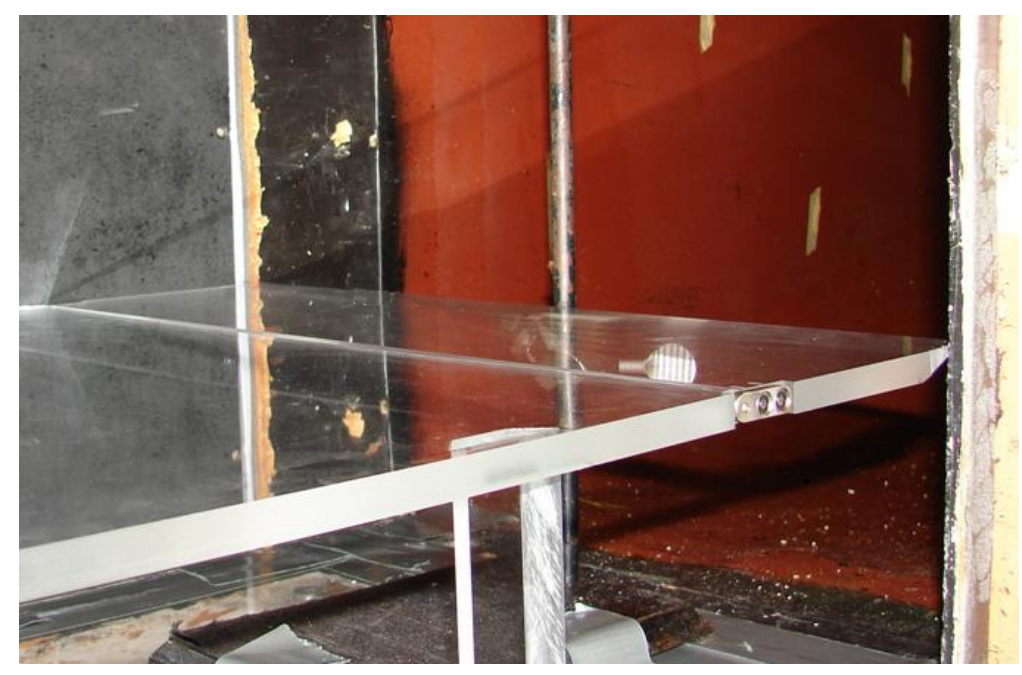

Figure 4-6 Trailing edge flap

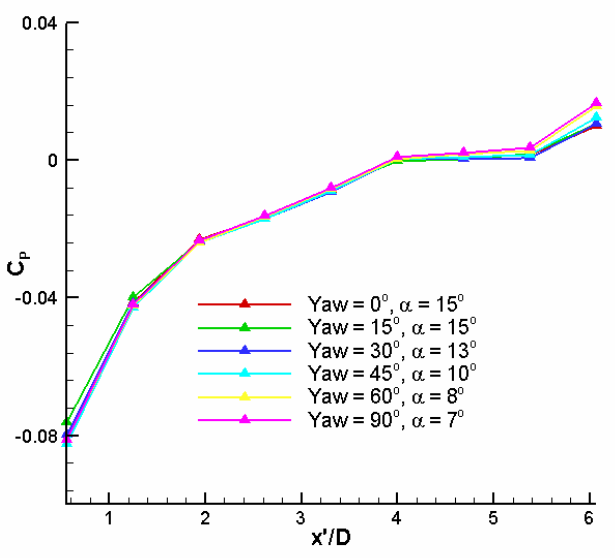

(a)

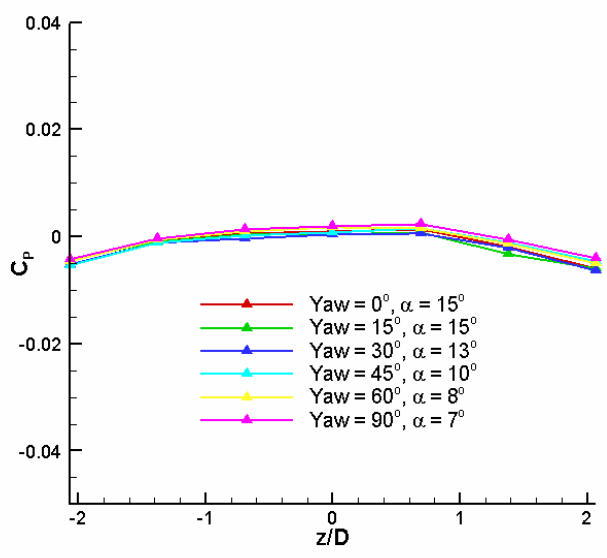

(b)

Figure 4-7 Plate surface pressure coefficient upstream of the cavity in streamwise (a) and spanwise directions (b) for different combinations of yaw angle and the flap angle ( $\alpha$ ) 


\section{- Roughness elements}

In order to generate experimental conditions similar to those of Hering [58] the same $\operatorname{Re}_{D}$ (Reynolds number, based on the minor axis length) and ratio between the thickness of the boundary layer upstream of the cavity and the length of the major axis $(\delta / \mathrm{D})$ should be established.

The nominal maximum velocity was $20 \mathrm{~m} / \mathrm{s}$ but at that velocity, tunnel flow was not steady. Therefore the experiments were carried out at the wind tunnel velocity of $18.3 \mathrm{~m} / \mathrm{s}$, resulting in $\mathrm{Re}_{\mathrm{D}}=8.7 \times 10^{4}$. This Reynolds number is slightly less than that of Hering and Savory's experiments $\left(9.1 \times 10^{4}\right)$, but of the same order of magnitude.

Also, the $\delta / \mathrm{D}$ ratio was 1.08 in Hering's experiments. To achieve the same ratio in the present experiments, a boundary layer thickness $(\delta)$ of $7.8 \mathrm{~cm}$ must be established at the upstream lip of the cavity. The natural turbulent boundary layer thickness at the cavity position, which was located $50 \mathrm{~cm}$ downstream the flat plate leading edge, can be estimated using the following formula [87]:

$$
\delta=0.37 x \operatorname{Re}_{x}^{-0.2}
$$

$\delta$ is equal to $1.3 \mathrm{~cm}$, which is comparable with the boundary layer thickness of $1.6 \mathrm{~cm}$ obtained based on the PIV experiments (Figure 4-8-(a)). Details of the PIV measurement are explained in section 4.6. The actual boundary layer was slightly thicker than predicted by Equation 4-3, possibly due to the garnet paper at the leading edge. The free stream turbulence intensity was around 2\% (Figure 4-8-(b)). However, this value is larger than the $1 \%$ value which was reported by Bailey [92]. This difference is possibly due to deterioration of flow quality in the tunnel over time.

A well-known approach for thickening the boundary layer is to use two- and threedimensional surface obstacles or roughness elements [99]. The height and location of surface obstacles should be chosen considering the fact the cavity should not be affected by the recirculation region generated by the roughness elements. The downstream 
recirculation region varies from 11-15 times the roughness element height, and it is affected by the depth of immersion of the obstacle into the boundary layer [100].

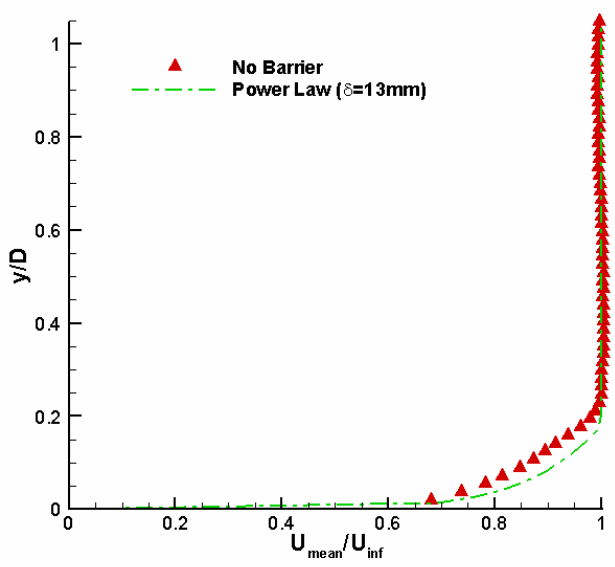

(a)

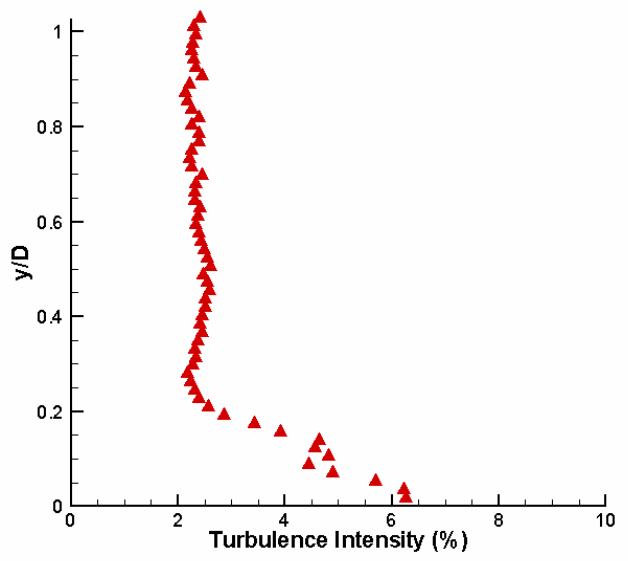

(b)

Figure 4-8 Velocity Profiles 1cm upstream of the cavity lip

Several combinations of different two- and three-dimensional roughness elements were used to find the best arrangement for the well-developed velocity profile with the proper boundary layer thickness at the upstream lip of the cavity. Two-dimensional obstacles with heights of $1.1,0.8,0.6$ and $0.3 \mathrm{~cm}$ were used, all of which had a width of $0.1 \mathrm{~cm}$. Hexagonal nuts with $0.5 \mathrm{~cm}$ height were used as three-dimensional roughness elements. (Figure 4-9).

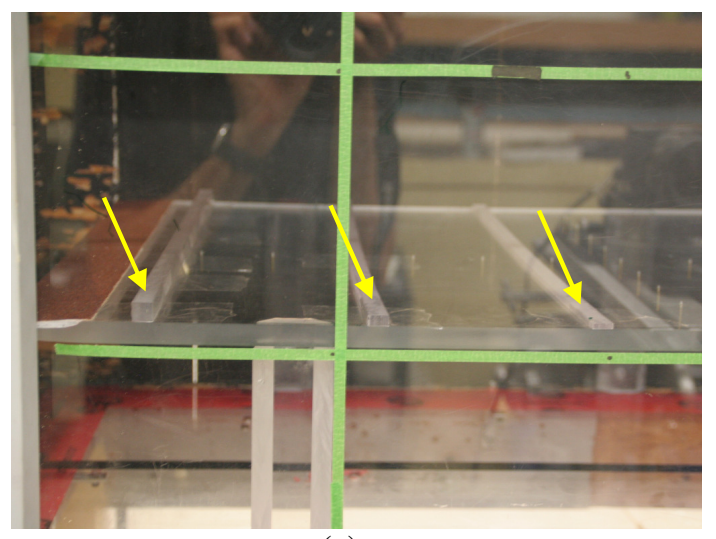

(a)

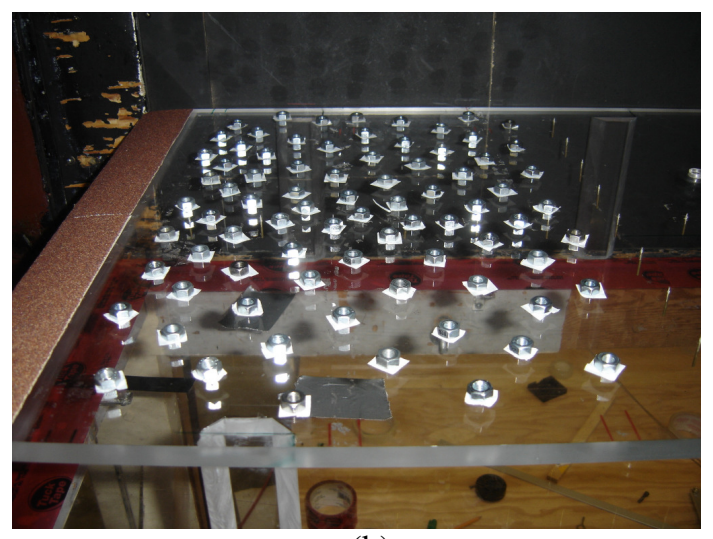

(b)

Figure 4-9 Two and three-dimensional obstacles 
The first approach for thickening the boundary layer was attaching one, two or three obstacles (descending in height) on the base plate. The first obstacle was attached to the surface immediately downstream of the garnet paper, and the next two were attached at equal intervals of $11 \mathrm{~cm}$ (Figure 4-9-(a)). The results (Figure 4-10) showed the following points:

- The boundary layer became thicker when the first obstacle was taller.

- The boundary layer was strongly affected by the height of the first obstacle height, whereas adding the shorter second and third obstacles did not change the velocity and turbulence intensity profiles significantly.

- Increasing the height of the first obstacle, led to deviation of the velocity profile from the power law velocity profile for the same turbulent boundary layer thickness (Figure 4-10-(b)). The reason for this was that the distance from the leading edge to the cavity lip was not long enough for velocity profile to become well-developed.

- Although the velocity profile was not changed very much by adding the second and third obstacles, adding them caused the velocity profile to deviate even further from that of a well-developed turbulent boundary layer.

The second approach was adding randomly placed nuts upstream and downstream of the cavity (Figure 4-9-(b)), and also combining them with 1.1 and $0.6 \mathrm{~cm}$ obstacles immediately after the garnet paper. In all three cases, adding the nuts led to profiles with a smaller velocity gradient compared to the cases where nuts were not used (Figure 4-10(a)).

Comparison of the results to those previously published in the literature, indicates that the same trend has been observed for velocity profiles downstream of a bluff plate (fence) with a splitter plate [101], rows of square plates [102], backward facing step [62], and two-dimensional obstacles [100] and [103]. Figure 4-11 shows velocity profiles downstream of a two-dimensional obstacle which are comparable to Figure 4-10-(b). 


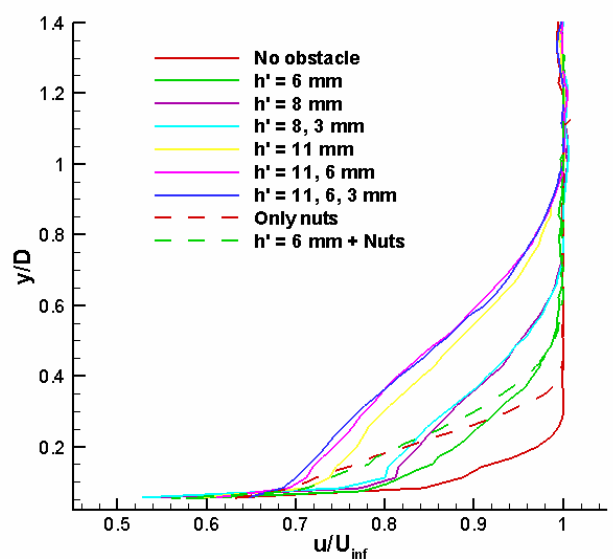

(a)

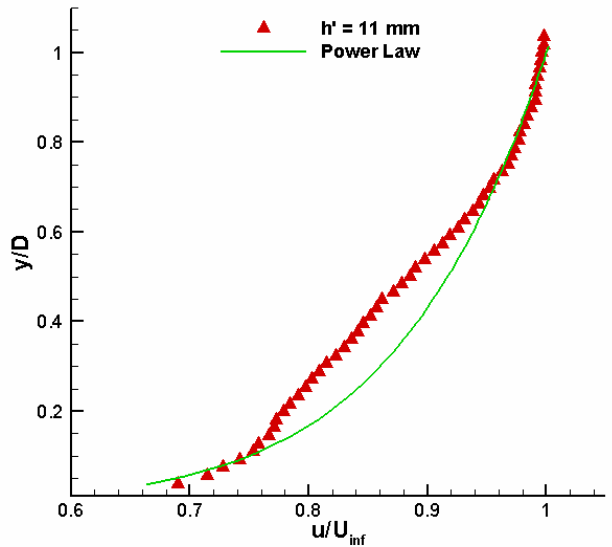

(b)

Figure 4-10 Velocity profiles comparison

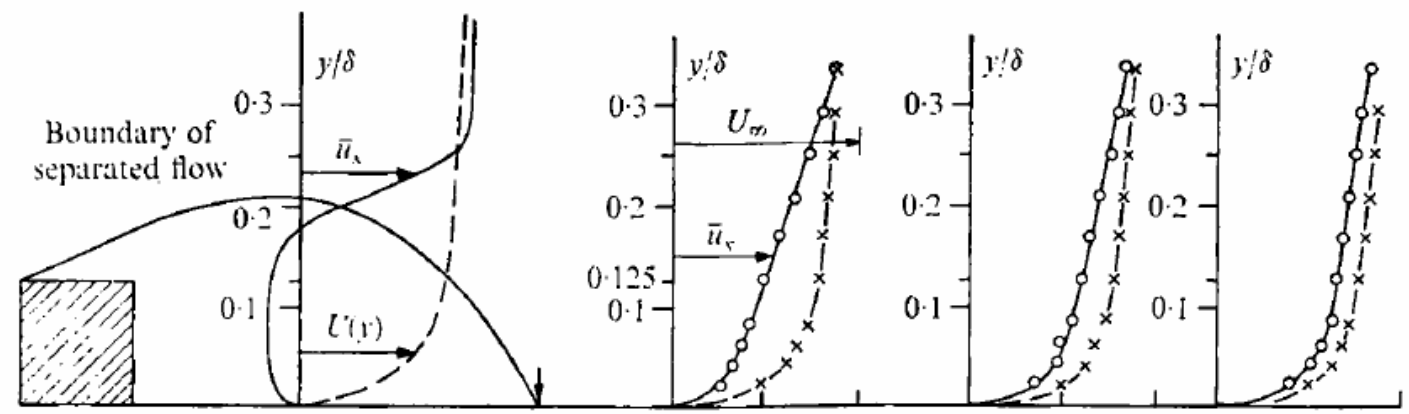

Figure 4-11 Velocity profiles downstream a two-dimensional obstacle, solid line shows experimental data and dashed line shows the power law profile [103]

It was concluded that the only solution to achieve a well-developed velocity profile of the desired thickness was a longer upstream length, which was not possible due to the limited length of the test section of the tunnel. Therefore, the combination that generated a velocity profile that best fitted the power law profile was chosen, although the boundary layer thickness was less than the value of $7.8 \mathrm{~cm}$, which was required to achieve the same $\delta / \mathrm{D}=1.08$ as Hering's experiments. The chosen velocity profile was obtained downstream of an obstacle of $0.6 \mathrm{~cm}$ height, with a $4.35 \mathrm{~cm}$ boundary layer thickness. The boundary layer parameters are explained in the next section. 


\subsection{Boundary Layer}

To have a proper boundary layer profile at the cavity location, two criteria should be met:

1. How closely the boundary layer profile matches that of a fully developed turbulent boundary layer

2. If the boundary layer can be considered as being two-dimensional (i.e. the same boundary layer profile at all spanwise locations)

One of the approaches to verify that the profile can be considered as a fully developed turbulent boundary layer over a flat plate, is matching of the friction velocity $\left(u_{*}\right)$ extracted from the experimental boundary layer data with that from the Law of the Wall approximation. Based on the experimental boundary layer data $u_{*}$ can be calculated using the following equation [85]:

$$
u_{*}=\sqrt{\frac{\tau_{w}}{\rho}}
$$

where, $\tau_{w}$ is the maximum wall shear stress measured in the region near the surface and equal to $-\rho \overline{u^{\prime} v^{\prime}}$ and $\rho$ is the density of the air. Therefore, $u_{*}=\sqrt{-\overline{u^{\prime} v^{\prime}}}$.

In order to determine the value of $u_{*}$ in the law of the wall formula, the experimental boundary layer velocity profile should be plotted in comparison with the Law of the Wall formula (Equation 4-5) [86]. Different values of $u_{*}$ in the law of the wall formula lead to different relative positions of the experimental profile and the law of the wall profile. There exists a $u_{*}$ value which causes the two profiles to match (Figure 4-12). If this value of $u_{*}$ matches the experimental value obtained using Equation 4-4, then the measured boundary layer profile can be considered as an empirical flat-plate turbulent boundary layer profile.

$$
\frac{\bar{u}}{u_{*}}=5.85 \log \frac{u_{*} y}{v}+5.56
$$




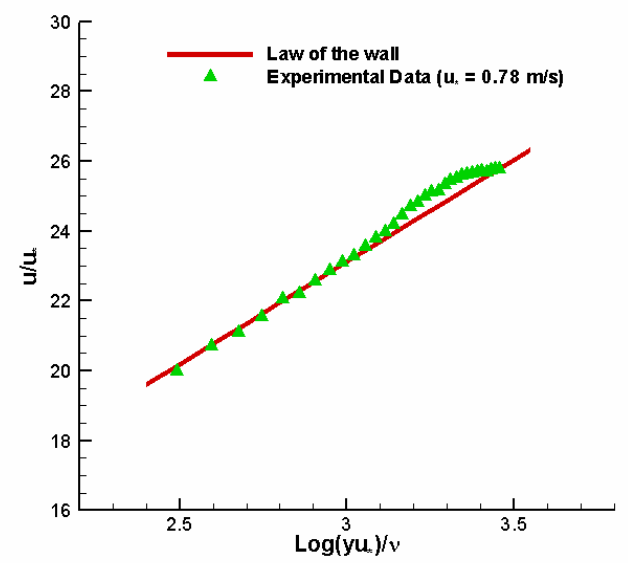

Figure 4-12 Comparison of the measured turbulent boundary layer data to the law of the wall

According to the calculations, the difference between these two values of $u_{*}$ is $4.9 \%$ which is within acceptable range and the profile can be considered as a fully developed turbulent boundary layer. Other parameters of the boundary layer are presented in Table 4-1. The value of shape factor $(H)$ is within the acceptable range for turbulent boundary layers [104] and [105].

The spanwise uniformity of the velocity profile was also checked. To do so, the measurements were carried out at seven parallel vertical planes upstream of the cavity lip, with a spanwise spacing of $50 \mathrm{~mm}$. The fourth plane was located in the centre of the plate $(\mathrm{z} / \mathrm{D}=0)$. The comparison of results showed that the velocity profile was similar at all spanwise locations. Figure 4-13 shows the comparison of dimensionless velocity and $\overline{u^{\prime} v^{\prime}}$ profiles, $10 \mathrm{~mm}$ upstream of the cavity lip. This figure shows similar profiles in the spanwise direction upstream of the cavity. The results presented in this section indicate that the flow upstream of the cavity includes a fully developed turbulent boundary layer with adequate spanwise uniformity, which meets both of the criteria mentioned at the beginning of this section. 
Table 4-1 Boundary layer parameters

\begin{tabular}{|l|c|}
\hline \multicolumn{1}{|c|}{ Parameter } & Value \\
\hline Freestream velocity $\left(\mathrm{U}_{\text {inf }}\right)$ & $18.3 \mathrm{~m} / \mathrm{s}$ \\
\hline Boundary layer thickness $(\delta)$ & $43.5 \mathrm{~mm}$ \\
\hline Displacement thickness $\left(\delta^{*}\right)$ & $4.55 \mathrm{~mm}$ \\
\hline Momentum thickness $(\theta)$ & $3.03 \mathrm{~mm}$ \\
\hline Shape factor $(\mathrm{H})$ & 1.5 \\
\hline Wall shear stress $\left(\tau_{w}\right)$ & $0.66 \mathrm{~Pa}$ \\
\hline Skin friction coefficient $\left(C_{f}\right)$ & 0.0035 \\
\hline Dimensionless friction velocity $\left(u_{*} / \mathrm{U}_{\mathrm{inf}}\right)$ measured from wall shear stress & $4.04 \times 10^{-2}$ \\
\hline Dimensionless friction velocity $\left(u_{*} / \mathrm{U}_{\mathrm{inf}}\right)$ from law of the wall & $4.26 \times 10^{-2}$ \\
\hline Difference in friction velocity measures & $4.9 \%$ \\
\hline
\end{tabular}

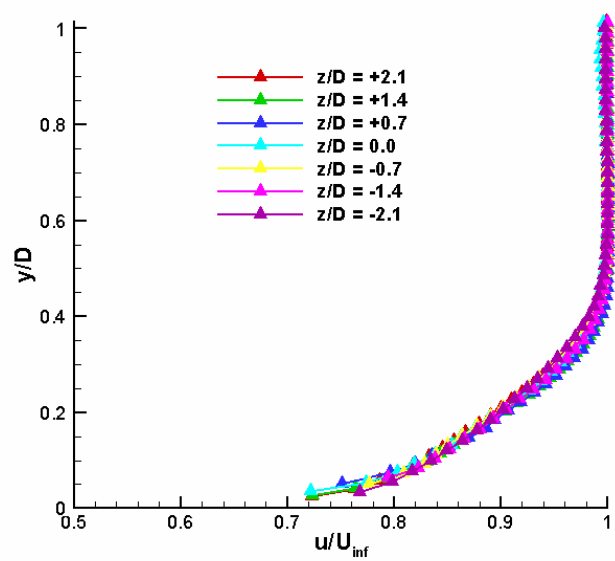

(a)

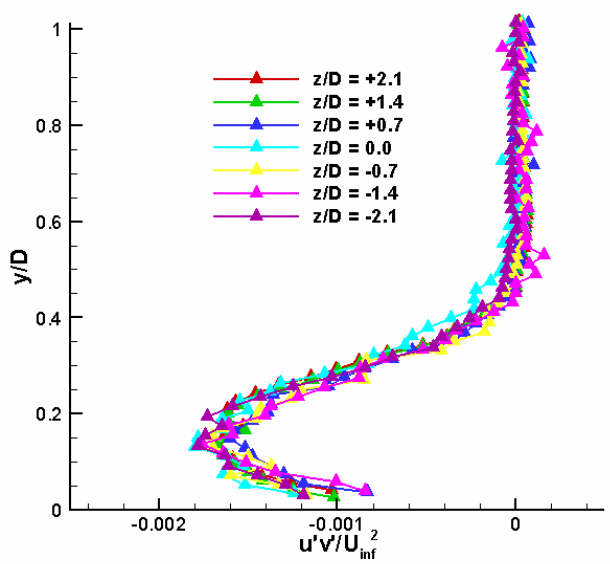

(b)

Figure 4-13 Comparison of dimensionless $\bar{u}$ profiles (a) and $\overline{u^{\prime} v^{\prime}}$ profiles (b) at seven upstream spanwise locations 


\subsection{Pressure Measurement Equipment}

For pressure measurement via pressure taps on the base plate, 15 linear differential pressure transducers were used. These pressure transducers were Honeywell DC001NDR4 and capable of measuring differential pressures between $\pm 25 \mathrm{~mm}$ of water $( \pm 250 \mathrm{~Pa})$. One port of these transducers was connected to the model pressure taps with \#16 PVC tubing and the other port was connected to static pressure port of the pitot-static tube. Also a pressure transducer with a $\pm 50 \mathrm{~mm}$ of water range was connected to the static and total ports of the pitot-static tube to measure the dynamic pressure of the freestream.

The pressure measurement signals were transmitted through a National Instruments SCB68 shielded connector block to a NI PCI-6052 data acquisition card. A LabVIEW code was used to coordinate between the sensing equipment and data acquisition system.

The pressure transducers were calibrated using a water-filled manometer as a reference. Nine different values of reference pressure were used to determine a calibration equation (linear fit) for each transducer. An example of a calibration graph obtained using this method for \pm 25 and $\pm 50 \mathrm{~mm}$ of water pressure transducers can be seen in Figure 4-14.

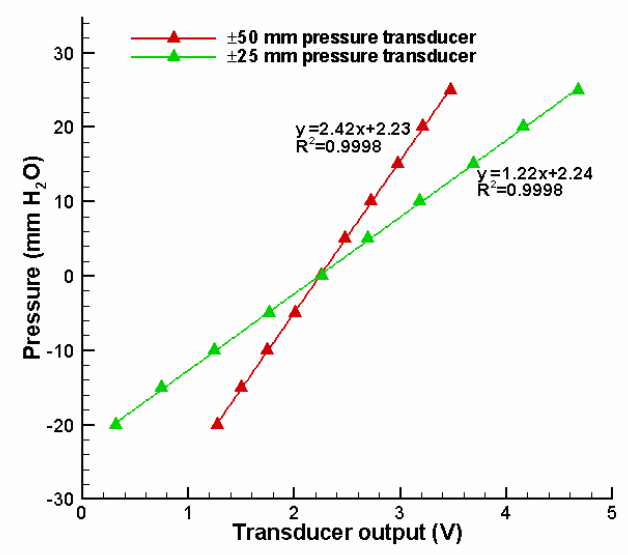

Figure 4-14 Example calibration curves for pressure transducers

The objective of these experiments was limited to determining the mean values of pressure at the measurement points, and measurement of possible unsteadiness in pressures was beyond the scope of the experiments. Considering this, a number of 
combinations of sampling rate and duration were tested, to determine the smallest number of samples beyond which the variations in mean values were negligible. Based on the results of these tests, the pressure taps were simultaneously sampled at $100 \mathrm{~Hz}$ for 10 seconds for each experiment.

Mean pressure coefficient $\left(\mathrm{C}_{\mathrm{p}}\right)$ values were calculated by using Equation 4-2. $P_{\text {static }}$ was measured at height of $15 \mathrm{~cm}$ above the base plate, $25 \mathrm{~cm}$ downstream of beginning of the test section and a distance of $15 \mathrm{~cm}$ from the side window. $\bar{P}-P_{\text {static }}$ for each location was measured by the $\pm 25 \mathrm{~mm}$ of water gauge pressure transducers and $P_{\text {dynamic }}$ was measured by the $\pm 50 \mathrm{~mm}$ of water gauge pressure transducer. The results of these measurements have been presented in Figure 4-7.

\subsection{PIV System}

Particle Image Velocimetry (PIV) is a non-intrusive technique which is used to obtain instantaneous velocity measurements and related flow properties such as turbulent characteristics of the flow with high spatial resolution.

In PIV measurement, a laser beam is expanded into a light plane using a cylindrical lens, and a spherical lens is used to compress the plane into a thin sheet. Then some tracer particles are seeded into the flow. A synchronizer acts as an external trigger for control of the camera and laser and two consecutive frames are captured within a very short time interval to obtain the light scattered by the particles from the laser pulses. Velocity vector fields are obtained by cross-correlating the interrogation window in one image to the search region in the other image in two dimensions [106].

As shown in Figure 4-15 and Figure 4-16 the PIV measurements were performed using:

- a CCD camera with resolution of $1600 \times 1200$ pixels with a 12 bit dynamic range operating in double exposure mode,

- a double pulse Nd-YAG laser with $120 \mathrm{~mJ} /$ pulse which generated a laser beam with a wavelength of $532 \mathrm{~nm}$, 
- a cylindrical lens with a focal length of $-15 \mathrm{~mm}$ to convert the laser beam to a light plane,

- different spherical lenses with various focal lengths of 500 or $1000 \mathrm{~mm}$, depending on the distance of the laser optics and the imaging area, to obtain the minimum thickness of the light plane at the imaging area,

- a computer equipped with built-in image buffer and timing unit, using TSI Insight $3 \mathrm{G}$ software for generation of vectors,

- a synchronizer, controlled by the computer to dictate the timing of each frame of the CCD camera's sequence in conjunction with the firing of the laser,

- A Laskin nozzle particle generator and olive oil to generate aerosol particles with average diameter of $2 \mu \mathrm{m}$ to seed the flow. The particles should be small enough so that response time of the particles to the motion of the fluid is reasonably short to accurately follow the flow, yet large enough to scatter a significant quantity of the incident laser light.

The size of the interrogation window was set to $32 \times 32$ pixels, and a $50 \%$ window overlap was used to increase the number of vectors. Considering the $1600 \times 1200$ pixel resolution of the images, an array of $99 \times 74$ velocity vectors was generated from each image pair. 


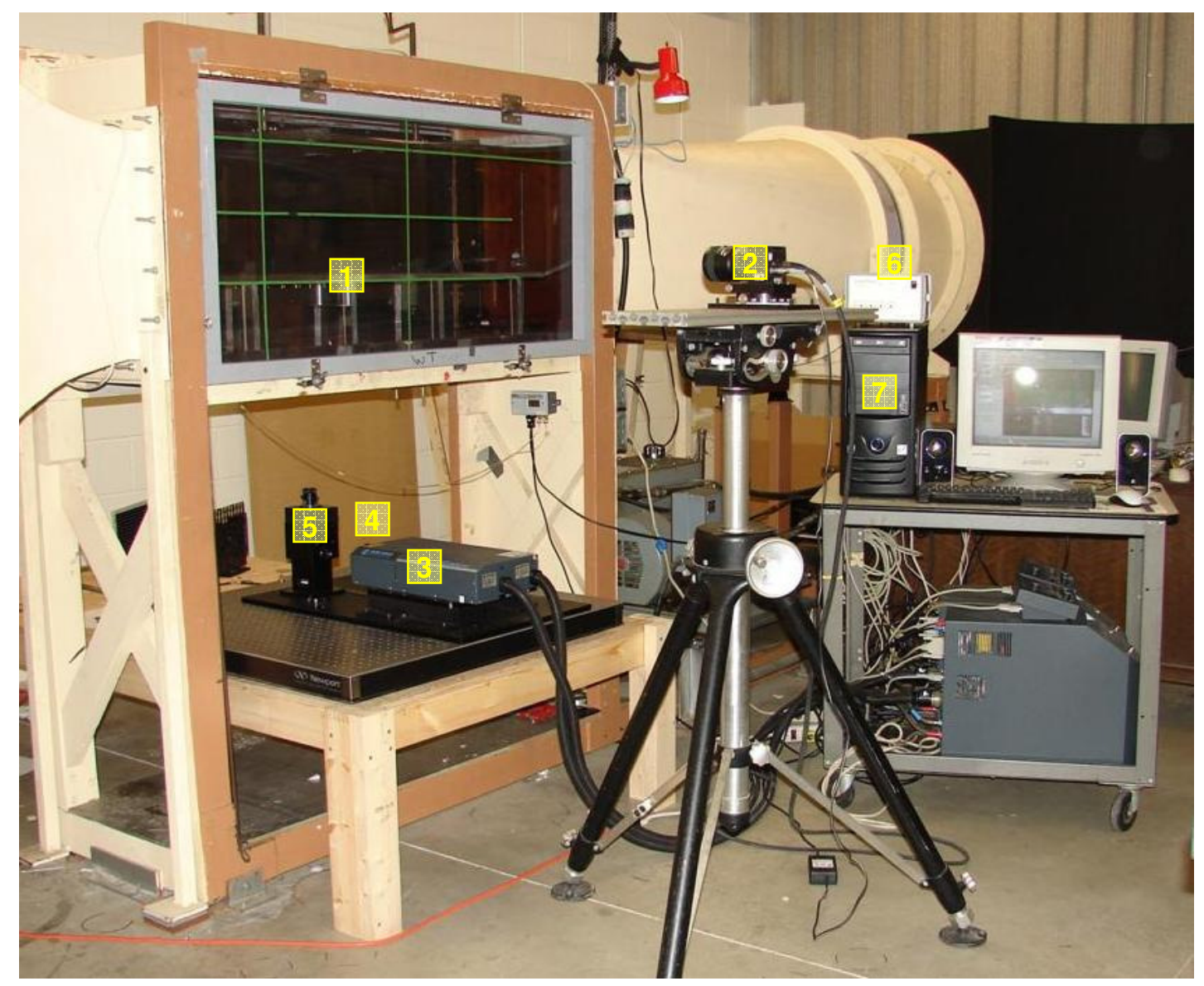

Figure 4-15 Images of the experimental setup, showing the main components: (1) model, (2) camera, (3) laser, (4) spherical lens, (5) laser beam reflector and cylindrical lens, (6) synchronizer, (7) imager recording computer

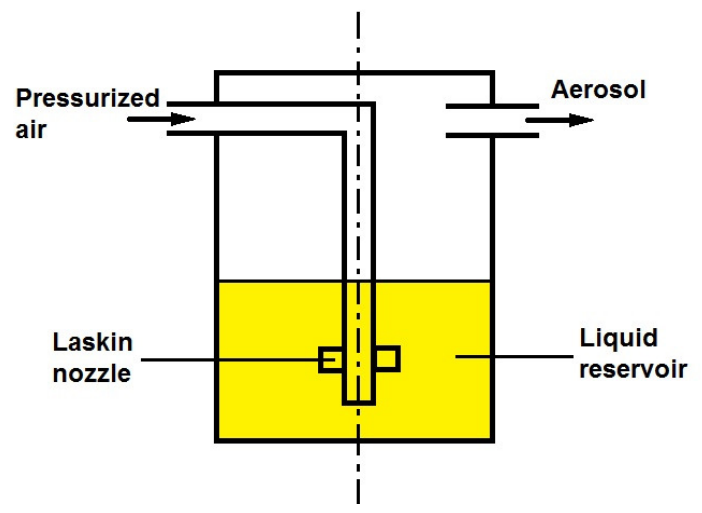

Figure 4-16 schematic Laskin nozzle aerosol generator 
The ability of seeding particles to follow the fluid flow is characterized by the Stokes number, which is given by Loth [107] as:

$$
S_{t}=\frac{\tau_{P}}{\tau_{f}}
$$

where $\tau_{P}$ is the response time of a seeding particle, and $\tau_{f}$ is any characteristic time scale of the fluid flow. When the Stokes number is much smaller than unity, the time required for the particle to assume the velocity of the fluid is negligible, and the particle can be assumed to have the same velocity as the fluid at any instance, which is the desirable case for PIV measurements. When the Stokes number is close to unity, the time lag between particle velocity and fluid velocity is no longer negligible, and when the Stokes number is larger than one, the particle velocity will not be affected by flow fluctuations. In both cases, the particle size is no longer appropriate for PIV measurements [107].

The response time of the particle is given in [107] as:

$$
\tau_{P}=\frac{\rho_{P} d_{P}{ }^{2}}{18 \mu}
$$

where $\rho_{P}$ and $d_{P}$ are density and diameter of the seeding particles, and $\mu$ is dynamic viscosity of air. For the particles used in the present study, which have an average diameter of $2 \mu \mathrm{m}$, and are made of light olive oil with $\rho_{P}=800 \mathrm{~kg} / \mathrm{m}^{3}$, approximately, the response time is $\tau_{P}=9.0 \times 10^{-6} \mathrm{sec}$.

Usually, the Stokes number is determined based on three characteristic time scales: $\tau_{f D}$, which is the time scale of macroscopic fluid motion, $\tau_{f \Lambda}$, which is the time scale of integral-scale turbulent motions, and $\tau_{f \lambda}$, which is the time scale associated with Kolmogorov scale, which is the scale of the smallest structures in a turbulent flow.

The macroscopic time scale can be estimated by the following equation [107]: 


$$
\tau_{f D}=\frac{D}{u_{D}}
$$

where $D$ and $u_{D}$ are the macroscopic characteristic length and velocity of the flow. For the present study, in which $u_{D}=\mathrm{U}_{\mathrm{inf}}=18.3 \mathrm{~m} / \mathrm{s}$, and $D=0.0725 \mathrm{~m}, \tau_{f D}=0.008 \mathrm{sec}$. This value leads to a Stokes number of 0.001, which is much smaller than unity, indicating that the seeding particles can follow the macroscopic fluid motions with negligible lag.

The integral-scale time scale can be estimated by the following equation [107]:

$$
\tau_{f \Lambda}=\frac{\Lambda}{u_{r m s}}
$$

where $\Lambda$ is the integral length scale. According to Piirto et al. [108], for an order of magnitude analysis similar to the one presented here, $\Lambda$ can be estimated to be $0.35 \mathrm{~h}$, where $\mathrm{h}$ is the smallest geometric length scale in the flow. Assuming $\mathrm{h}=0.006 \mathrm{~m}$, which is the height of the two-dimensional obstacles used upstream of the cavity, and approximate value of $0.002 \mathrm{~m}$ is obtained for $\Lambda$, leading to a time scale of $\tau_{f \Lambda}=9.5 \times 10^{-4} \mathrm{sec}$, based on a typical value of $u_{\mathrm{rms}}=2.2 \mathrm{~m} / \mathrm{s}$. This time scale results in a Stokes number of 0.0094, approximately, which is much smaller than unity, and indicates that the seeding particles can follow the fluid motion at the integral scale with negligible lag.

An estimate for the time scale associated with Kolmogorov scale can be obtained by the following equation [107]:

$$
\tau_{f \lambda}=\frac{\lambda}{u_{\lambda}}
$$

in which $\lambda=\left(\frac{v^{3}}{\varepsilon}\right)^{1 / 4}$ and $u_{\lambda}=\left(v^{3} \varepsilon\right)^{1 / 4}$ are the Kolmogorov length and velocity scales, repectively. In the above equations, $v$ is kinematic viscosity, and $\varepsilon$ is turbulent dissipation rate, which can be estimated using the following equation [108]: 


$$
\varepsilon=\frac{u_{r m s}^{3}}{\Lambda}
$$

For the present study, the above equation gives a value of $5.07 \times 10^{3}$ for turbulence dissipation rate, leading to the values of $2.86 \times 10^{-6} \mathrm{~m}$ and $0.064 \mathrm{~m} / \mathrm{s}$ for $\lambda$ and $u_{\lambda}$, respectively. Based on these values, the time scale associated with Kolomogorov scale is found to be $4.44 \times 10^{-5} \mathrm{~m} / \mathrm{s}$, which results in a Stokes number of 0.2. This value of Stokes number is close to unity, indicating that the particle size in the present study is not appropriate for following the turbulent fluid motions at the Kolmogorov scale.

\subsection{Flow Visualization}

Different methods such as smoke wire, tufts, dry ice, and helium bubbles were used to visualize the flow inside and downstream of the cavity. The first three methods were found not to be appropriate for this experimental setup and flow regime.

The problem with the smoke wire was that the smoke was not entrained into the cavity. The reason was that the smoke rose from the plate surface due to the two-dimensional obstacle at the leading edge of the base plate and because of its lower density compared to air. It did not reattach to the surface downstream of the obstacle and passed the cavity at same distance from it.

The second approach was using tufts. The problem with this method was that the velocity inside the cavity was low compared to the free-stream velocity. Therefore, the longer and heavier tufts were not moved very well by the flow and were tangled into each other, whilst the shorter tufts remained mainly in the direction in which they were attached to the surface rather than the flow direction.

The third method was dry ice. The dry ice had to be vaporized by pouring water on it. There were two possibilities regarding the dry ice location. It could be placed either upstream of the cavity, in which case it would disturb the upstream flow and the water used to vaporize it turned into ice after a very short period of time. The dry ice could also be put inside the cavity, in which case it not only made a disturbance and a change in the depth of the cavity, but also the glue which was used to attach the cavity bottom to the 
cavity walls could not tolerate the low temperature and detached immediately (Figure 4-17).

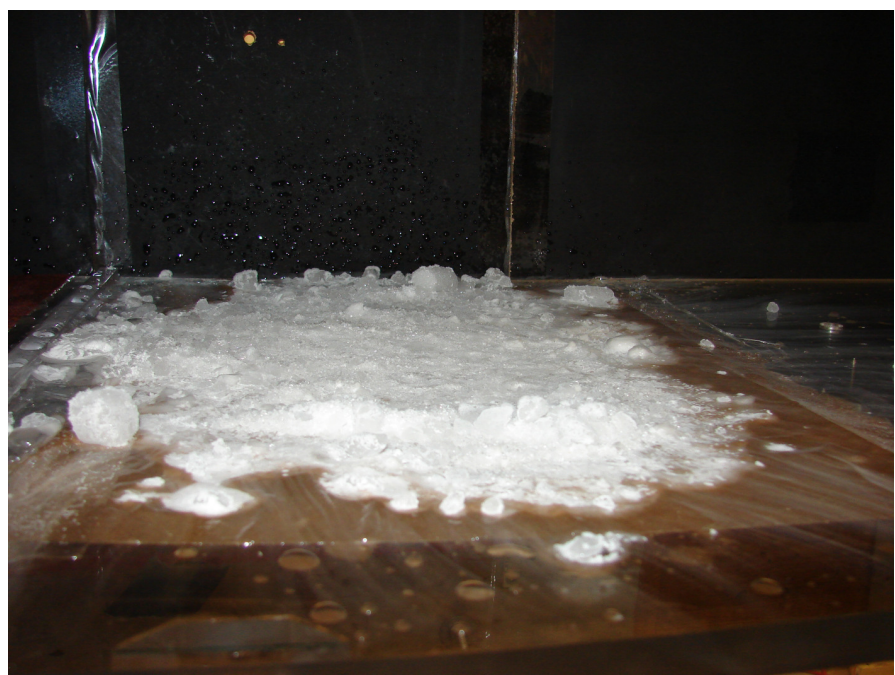

(a)

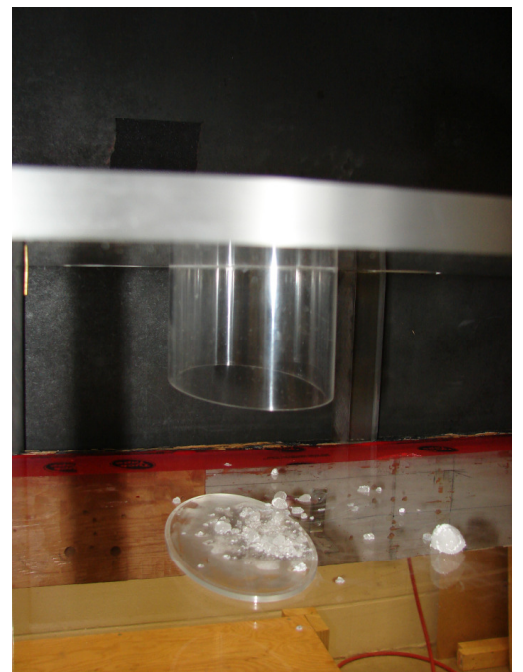

(b)

Figure 4-17 Dry ice placed at the upstream of the cavity (a) and inside the cavity (b)

The last method, which was used successfully, was helium bubbles. For this purpose $\mathrm{SAI}^{\mathrm{TM}}$ modified model 5 Helium bubble generator was used (Figure 4-18-(a)). Heliumfilled, almost neutrally-buoyant bubbles of uniform size, adjustable from $0.8 \mathrm{~mm}$ to $5 \mathrm{~mm}$ in diameter, could be generated simultaneously from one or two outputs.

The Plug-in head, shown in Figure 4-18-(b) consisted principally of a concentric arrangement of two stainless steel hypodermic tubes, one inside the other, attached in a cantilever fashion to a cylindrical manifold base or body. Within the head, helium passed through the inner hypodermic tube and "Bubble Film Solution" (BFS) through the annulus between the inner tube and the outer tube to form the helium-filled bubbles at the tip. A much larger concentric jet of air blew the bubbles continuously off the tip. To operate the system external sources of helium and compressed air were required. A cylinder of compressed helium with a 0 to $207 \mathrm{kPa}$ pressure regulator was used and compressed air at $414 \mathrm{kPa}$ was delivered to the system. The results of flow visualization using this technique are presented in Chapter 5. 


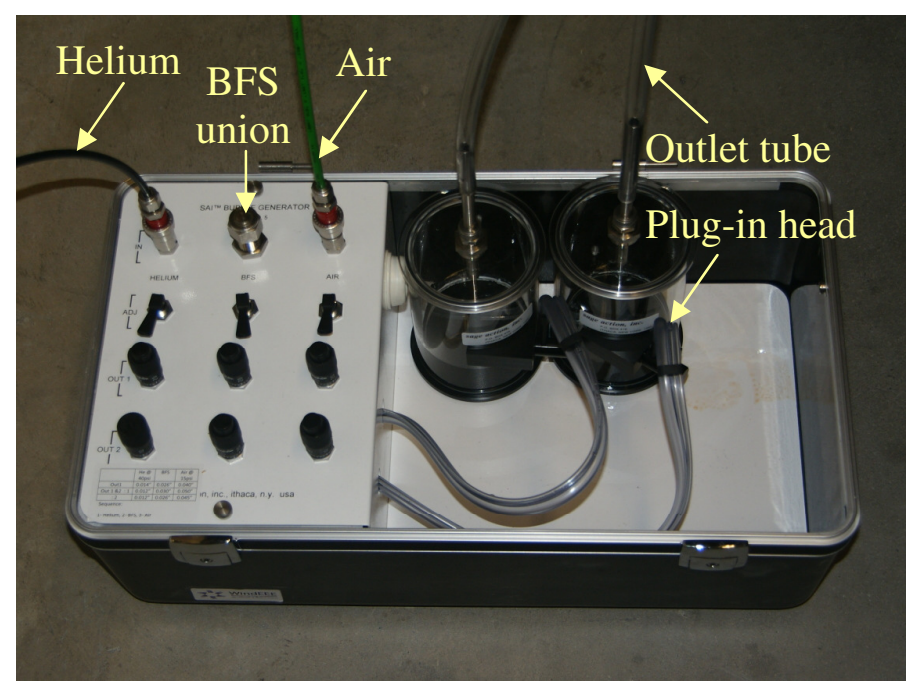

(a)

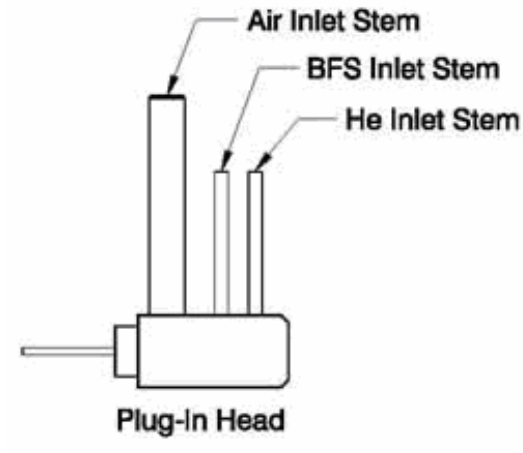

(b)

Figure 4-18 Helium bubble generator

\subsection{PIV Measurement Planes and Convergence Test}

To be able to capture flow phenomena inside the cavity, measurements were carried out in several vertical $(x-y)$ and horizontal $(x-z)$ planes to measure the respective velocity components. Also, wake measurements were carried out in two horizontal (x-z) planes downstream of the cavity.

For all the cases, regardless of the yaw angle and cavity depth, five vertical planes were chosen to carry out the measurements. The third plane was always located at $\mathrm{z} / \mathrm{D}=0$. Since a lower yaw angle means a longer spanwise length of the cavity, the spacing of the vertical planes spacing was larger at lower yaw angles compared to the higher ones. The locations of these vertical planes for each yaw angle are presented in Table 4-2.

Table 4-2 The locations of vertical planes for different yaw angles

\begin{tabular}{|c|c|}
\hline Yaw angle (degrees) & z/D \\
\hline 0,15 and 30 & $\pm 0.70, \pm 0.35$, and 0 \\
\hline 45 and 60 & $\pm 0.53, \pm 0.26$, and 0 \\
\hline 90 & $\pm 0.35, \pm 0.18$, and 0 \\
\hline
\end{tabular}


For horizontal plane measurements, the number of planes was independent of the yaw angles and depended only on the cavity depth. The lowest plane was at located $1 \mathrm{~cm}$ above the cavity floor, and the next planes were located in $1 \mathrm{~cm}$ intervals. The only exception was the last (highest) horizontal plane, for which the location was chosen individually in each case to provide optical accessibility. Table 4-3 shows the locations of the horizontal planes for each cavity depth.

For measurements in the wake area, two horizontal planes were chosen. The planes were located at heights of $3 \mathrm{~mm}$ and $6 \mathrm{~mm}$ from the surface of the ground plane, downstream of the cavity.

Table 4-3 The locations of horizontal planes for different cavity depths

\begin{tabular}{|c|c|}
\hline $\mathrm{h} / \mathrm{D}$ & $\mathrm{y} / \mathrm{D}$ \\
\hline 1.0 & $-0.86,-0.72,-0.59,-0.45,-0.31$, and -0.22 \\
\hline 0.7 & $-0.56,-0.42,-0.29$, and -0.20 \\
\hline 0.5 & -0.36 and -0.22 \\
\hline
\end{tabular}

To determine the numbers of samples (image pairs) that should be recorded to obtain results independent of the number of samples, several tests with different number of samples were carried out. The mean and standard deviation of velocity were compared for different numbers of samples. Qualitative comparison was carried out based on plots of mean and standard deviation of velocity in the measurement plane (an example of which is shown in Figure 4-19). Furthermore, distributions of mean and standard deviation of velocity along a horizontal line located at the mid-depth of the cavity, and a vertical line located at $\mathrm{x} / \mathrm{D}=0$, on the mid-plane $(\mathrm{z} / \mathrm{D}=0)$ of the cavity with $\mathrm{h} / \mathrm{D}=1.0$ at the yaw angle of $90^{\circ}$ were used for quantitative comparison. The objective of this quantitative comparison was to determine the smallest number of samples beyond which increasing the number of samples did not result in significant changes in the quantitative values. A maximum variation of $1.5 \%$ in mean and standard deviation of velocity was chosen as a quantitative criterion. Results of this quantitative comparison are presented in 
Table 4-4. The results indicate that increasing the number of samples from 2000 to 3000 results in less than $1.5 \%$ variations in both mean and standard deviation of velocity. Based on these results, the number of the samples to be recorded was set to 2000 for all measurement cases.

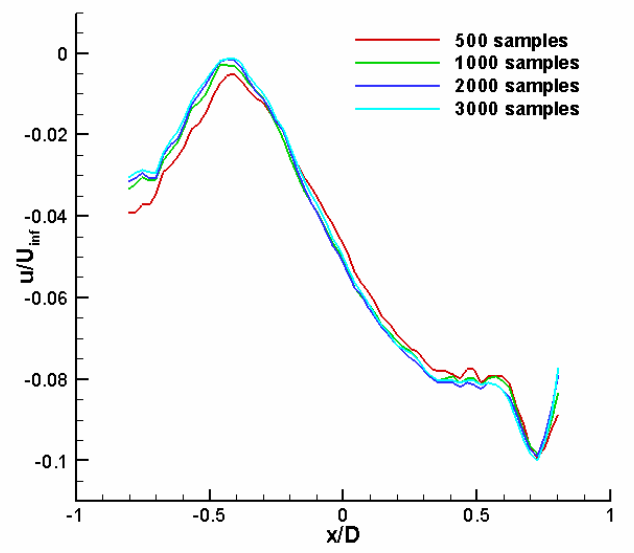

(a)

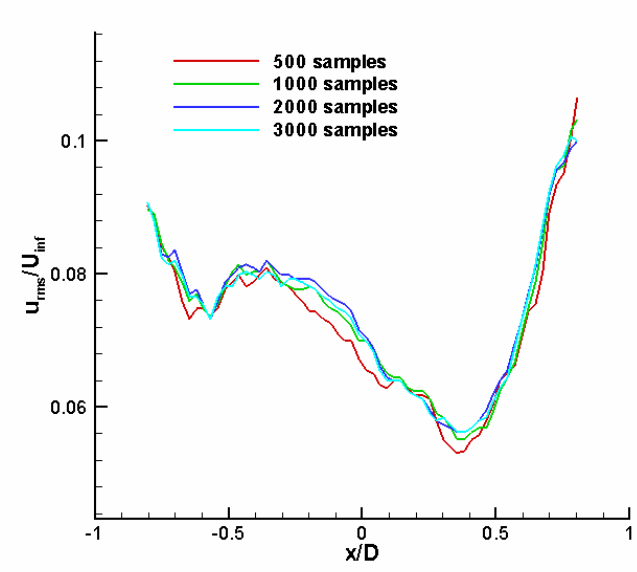

(b)

Figure 4-19 Effect of the number of samples on distributions of $\bar{u}$ (a) and $\boldsymbol{u}_{r m s}$ (b) on a horizontal line located at $\mathrm{z} / \mathrm{D}=\mathbf{0}$ and $\mathrm{y} / \mathrm{D}=\mathbf{- 0 . 6}$, for the cavity with $\mathrm{h} / \mathrm{D}=1.0$ at yaw angle $=90^{\circ}$

Table 4-4 Average variations of $\bar{u}$ and $u_{r m s}$ due to increasing the number of samples

\begin{tabular}{|c|c|c|c|c|c|}
\hline \multicolumn{2}{|c|}{ Number of samples } & 500 & 1000 & 2000 & 3000 \\
\hline \multirow{3}{*}{$\mathrm{y} / \mathrm{D}=-0.5$} & Average variation of $\bar{u}(\%)$ & - & 2.8 & 1.5 & 1.2 \\
\cline { 2 - 6 } & Average variation of $u_{r m s}(\%)$ & - & 2.2 & 1.6 & 0.9 \\
\hline \multirow{2}{*}{$\mathrm{x} / \mathrm{D}=0$} & Average variation of $\bar{u}(\%)$ & - & 2.8 & 1.1 & 1.0 \\
\cline { 2 - 6 } & Average variation of $u_{r m s}(\%)$ & - & 2.0 & 1.3 & 0.6 \\
\hline
\end{tabular}

\subsection{Experimental Uncertainty}

\subsubsection{Pressure Transducers}

Dybenko [109] has carried out an extensive error analysis for the DC001NDR4 pressure transducers which were used for the present experiments. The sources of errors identified 
are temperature effect and drift over the length of trial, linearity and hysteresis, and calibration. The root of the sum of the squares (RSS) method was applied in order to estimate the total error. According to this method, for adding multiple random error sources, the uncertainty to $95 \%$ confidence was calculated using the following equation [110]:

$$
w_{95}=\sqrt{\sum_{i=1}^{n}\left(\Delta E_{i}\right)^{2}}
$$

where, $i$ is the index for individual random error sources, $n$ is the total number of random error sources to be summed, and $\Delta E$ is a source of uncertainty as a fraction of the nominal value of the quantity measured. Results of the analysis by Dybenko [109] indicate that the error magnitude relative to nominal measurement for a typical $C_{p}$ value of 0.15 is $\pm 2.28 \%$.

\subsubsection{PIV System}

The uncertainty associated with measurement of mean and fluctuating velocities, and Reynolds stresses, using the PIV technique is comprised of two components. One component is the measurement error, also known as bias, which is caused by various aspects of the measurement technique and equipment. The other component is the statistical uncertainty, also known as random error, which is due to the statistical variation of the measured quantity in multiple measurements. These two components will be estimated in the following paragraphs.

There are several sources for PIV measurement error. These uncertainties can be resulted by velocity gradients, particle image size, out of plane motion, peak locking, and interpolation [111]. The error due to each of these parameters has been investigated and the total measurement error has been calculated accordingly.

- Velocity gradients

The mean velocity gradients can be calculated based on the results of PIV measurements in various combinations of measurement planes, h/D ratio, and yaw 
angle. A typical estimate of the mean velocity gradient, based on the results of PIV measurements for a representative case $\left(\mathrm{h} / \mathrm{D}=1.0\right.$, yaw angle $\left.=90^{\circ}\right)$ is used for error estimation. For this case, the normalized mean velocity gradients in the streamwise (x), vertical (y), and spanwise (z) directions are found to be $0.6 \%$ and $0.3 \%$, and $0.6 \%$, respectively, based on the data in vertical (x-y) and horizontal $(\mathrm{x}-\mathrm{z})$ planes. The error associated with velocity gradient can be determined using Figure 5(e) of Reference [111]. The figure indicates an error of 0.03 pixels, approximately, due to the mentioned velocity gradients.

- Particle image size

An investigation of the PIV images of the present experiments indicates that the size of the image of most of the particles in the field of view is 1 pixel. This particle image size leads to an error of 0.08 pixels, based on Figure 5(a) of Reference [111].

- Out of plane motion

The thickness of the laser light sheet in the present experiments is approximately $1 \mathrm{~mm}$ in the measurement area. This thickness is equivalent to 8.5 pixels, approximately, based on the calibration coefficient in both vertical (x-y) and horizontal ( $\mathrm{x}-\mathrm{z})$ planes. The thickness of the laser light sheet is therefore larger than the average in-plane pixel displacement of the present experiments, which is around 5 in most cases. Assuming that the out of plane pixel displacement is less than the in plane displacement, it can be concluded that the error due to out of plane motion of the particles is negligible.

- Peak locking

The term peak locking is used to describe the biasing of particle displacements toward integer pixel values. Peak locking is a result of both the choice of subpixel fit estimator, and under-resolved optical sampling of the particle images [112]. The RMS velocities and the Reynolds stress are sensitive to peak locking, however, the mean velocity profiles are insensitive to this effect [112]. Although 
various sub-pixel fit estimators have been proposed, Westerweel has shown that a Gaussian sub-pixel estimator is capable of reducing the effect of peak locking significantly, compared to other methods [113]. Since this is the method that has been implemented for correlation peak estimation in the Insight 3G PIV software, the peak-locking error has been assumed to be negligible.

- Interpolation

In PIV measurement, it is required to interpolate the randomly located data onto a grid in order to calculate turbulent statistics. According to Cower and Monismith's work, the error due to interpolation is almost constant and equal to 0.08 pixels [111].

By using the root of the sum of the squares (RSS) method [110], the total uncertainty of PIV measurements due to the above mentioned factors is 0.12 pixels approximately, which can be converted to a velocity magnitude using time intervals and calibration factor for each case. As a representative case, in mid-plane of the cavity with $\mathrm{h} / \mathrm{D}=1.0$ at yaw angle of $90^{\circ}$, which has the largest measurement area among all of the cases in the present study, this value translates into an uncertainty of $0.29 \mathrm{~m} / \mathrm{s}$ in terms of velocity.

The statistical uncertainty can be determined based on the approach described in [114]. In this approach, the following equation is used for estimating the statistical uncertainty, assuming a large population with normal distribution:

$$
e=\frac{z \sigma_{x}}{\sqrt{N}}
$$

where, $N$ is the number of independent measurements, $\sigma_{x}$ is the standard deviation of the quantity for which the statistical error is calculated, $e$ is the absolute uncertainty, and $z$ denotes the probability of estimating the mean value within $\pm e$. For example, $z=1.96$ for a probability of $95 \%$ for estimating mean value within $\pm e$.

Based on the above-mentioned equation, the absolute statistical uncertainty in each of the two velocity components $(\bar{u}$ and $\bar{v})$ for a single measurement (snapshot) in the 
representative case of the cavity with $\mathrm{h} / \mathrm{D}=1.0$ at $90^{\circ}$ yaw angle, is found to be $e_{\bar{u}}=z u_{r m s}=2.74 \mathrm{~m} / \mathrm{s}$ and $e_{\bar{v}}=z v_{r m s}=1.37 \mathrm{~m} / \mathrm{s}$, based on a value of 1.96 for $z$.

These values, which are based on the maximum root mean square velocity components in the cavity in the above-mentioned case, can then be combined with the absolute measurement error of the PIV system, using the root of the sum of the squares (RSS) method [110], according to Equation 4-14. The resulting total uncertainty for a single measurement (snapshot) are $e_{\bar{u} \text { Total }}=2.76 \mathrm{~m} / \mathrm{s}$ and $e_{\bar{v} \text { Total }}=1.40 \mathrm{~m} / \mathrm{s}$.

$$
e_{\text {total }}=\sqrt{e_{\text {random }}^{2}+e^{2} \text { measurement }}
$$

Based on the values of total uncertainty for a single measurement, the absolute value of the total uncertainty in the components of mean velocity for 2000 independent measurements can be calculated using Equation 4-13. Benedict and Gould [115] have expanded this approach, and provided formulations to estimate the uncertainty in certain other variables in a turbulent flow. This formulation has been applied to calculate the uncertainties in the mean and fluctuating velocities, and Reynolds stresses in the present experiments. The results of these calculations are summarized in Table 4-5. In all calculations, $N=2000$ and $z=1.96$.

Table 4-5 Total uncertainty of flow variables

\begin{tabular}{|c|c|c|c|c|}
\hline Flow parameter & Absolute uncertainty & Units & Reference value & Relative uncertainty \\
\hline $\bar{u}$ & 0.062 & $\mathrm{~m} / \mathrm{s}$ & 5 & $1.2 \%$ \\
\hline $\bar{v}$ & 0.031 & $\mathrm{~m} / \mathrm{s}$ & 5 & $0.6 \%$ \\
\hline$u_{r m s}$ & 0.044 & $\mathrm{~m} / \mathrm{s}$ & 1.4 & $3.1 \%$ \\
\hline$v_{r m s}$ & 0.022 & $\mathrm{~m} / \mathrm{s}$ & 0.7 & $3.2 \%$ \\
\hline$\overline{u^{\prime} u^{\prime}}$ & 0.122 & $\mathrm{~m}^{2} / \mathrm{s}^{2}$ & 1.96 & $6.2 \%$ \\
\hline$\overline{v^{\prime} v^{\prime}}$ & 0.031 & $\mathrm{~m}^{2} / \mathrm{s}^{2}$ & 0.49 & $6.4 \%$ \\
\hline$\overline{u^{\prime} v^{\prime}}$ & 0.061 & $\mathrm{~m}^{2} / \mathrm{s}^{2}$ & 0.72 & $8.5 \%$ \\
\hline
\end{tabular}


Another point to be noted with regards to PIV measurements in vertical planes inside the cavity is the curvature of the surface and the resulting image distortion in the horizontal direction. The cavity wall was manufactured as thin as practically possible $(3 \mathrm{~mm})$ to reduce the image distortion, and image distortion was measured quantitatively using a grid of uniform points, placed inside the cavity. The least curved surface (facing the camera) belongs to yaw angle $=90^{\circ}$ (Figure 4-20-(a)). In this case, the maximum image distortion, which occurs in a narrow band at both corners, is $3 \%$. In the remaining part of the cavity, image distortion is less than $1 \%$. The most curved surface (facing the camera) belongs to yaw angle $=0^{\circ}$ (Figure 4-20-(b)). In this case the image distortion is $4 \%$ and the value is almost constant over the entire length of the cavity.

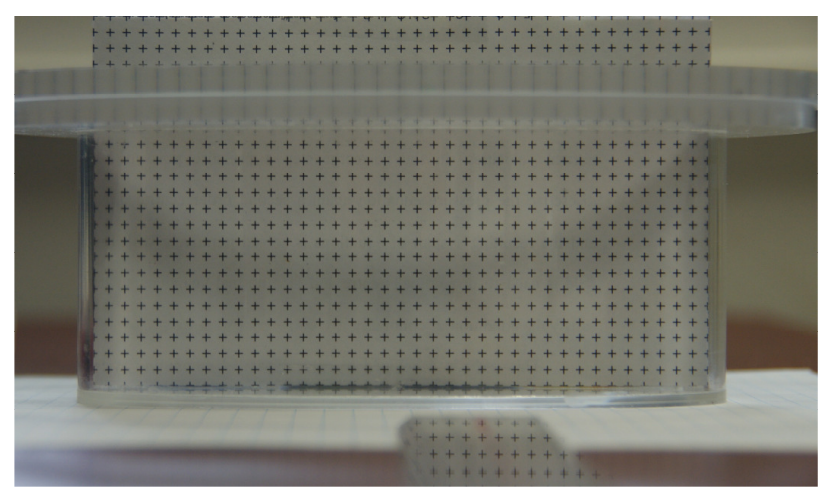

(a)

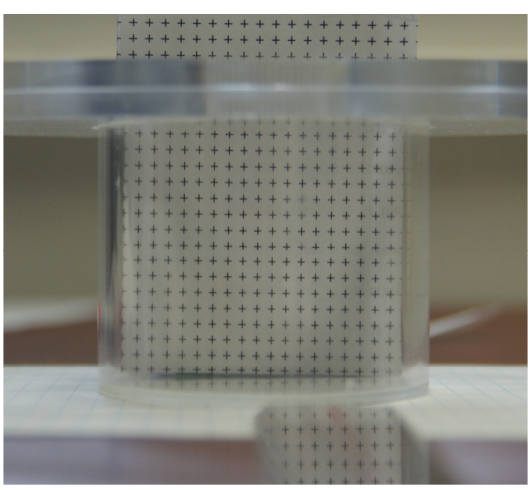

(b)

Figure 4-20 Image distortion for yaw angle $=90^{\circ}$ (a) and yaw angle $=0^{\circ}(\mathrm{b})$

\subsection{Reference Velocity}

The other important aspect of velocity profile comparison is the choice of an appropriate reference velocity $\left(U_{\text {ref }}\right)$ for normalizing the results. The first candidate for reference velocity is the freestream velocity $\left(U_{\text {inf }}\right)$. However, it should be noted that although the freestream velocity is the characteristic velocity of the flow, the cavity is not exposed to the freestream velocity directly. In practice, the velocity at the upper edge of the cavity, is related to the freestream velocity through the separated shear layer. As a result, the velocity distribution inside the cavity is strongly affected by the conditions of the shear layer, including its thickness and velocity gradient. One of the parameters affecting the condition of the shear layer along the upper edge of the cavity, and therefore the 
distribution of flow parameters inside the cavity is the upstream boundary layer characteristics. The effect of the thickness and state of the upstream boundary layer on the flow past the shear layer separation point is discussed earlier in Chapter 2.

As mentioned previously, the upstream boundary layer thickness in the experiments is different from that of the numerical simulations $(\delta / \mathrm{D}=0.6$ and 1.08 for the experiments and the numerical simulations). If $U_{\text {inf }}$ is used to normalize the profiles, the effects of the boundary layer thickness will not be accounted for. As a result, although experimental and numerical profiles normalized using $U_{\mathrm{inf}}$ would follow the same trend qualitatively, they would not match quantitatively.

Savory et al. have used the shear velocity $\left(u_{*}\right)$ as reference velocity to normalize pressures inside the cavity [48]. Using the shear velocity as reference accounts for the effects of upstream boundary layer thickness, and proves to be suitable for normalizing pressure distributions. The present results indicate that cases with similar values of $u_{*}$ in the upstream boundary layer can have different velocity distributions inside the cavity. This behaviour is illustrated in Figure 4-21. This figure shows the difference between the velocity distribution in the shear layer, for two cavities with similar upstream boundary layers, but different yaw angles. The figure compares the velocity profiles at the top of the cavity, for the cavity with $\mathrm{h} / \mathrm{D}=1.0$, at two extreme cases of $0^{\circ}$ and $90^{\circ}$ yaw angles, based on the CFD results. For each case, velocity profiles have been shown in the midplane, at a location $0.05 \mathrm{D}$ after the upstream cavity edge $\left(\mathrm{x} / \mathrm{D}=-0.45\right.$ for $0^{\circ}$ yaw and $\mathrm{x} / \mathrm{D}$ $=-0.95$ for $\left.90^{\circ}\right)$, and the centre of the cavity $(x / D=0)$.

Comparison of the profiles at $\mathrm{x} / \mathrm{D}=-0.45$ for yaw angle $=0^{\circ}$ and $\mathrm{x} / \mathrm{D}=-0.95$ for yaw angle $=90^{\circ}$ shows that the velocity magnitudes and gradients are similar right after the flow enters the cavity. Comparison of the two profiles at $\mathrm{x} / \mathrm{D}=0$ shows considerably different velocity profiles. The difference in velocity profiles may be attributed to two effects. One is the effect of the separated shear layer with respect to the upper edge of the cavity, and the other is the thickness of the shear layer. These effects will be investigated in the following paragraphs. 


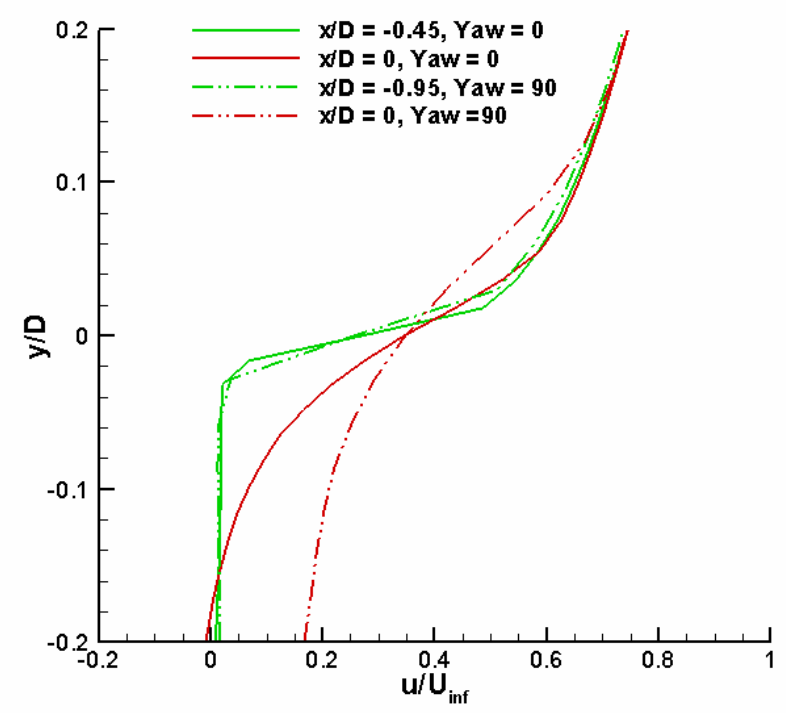

Figure 4-21 Velocity profile comparison for two different yaw angles at two different locations

The centre of the shear layer can be determined by finding the location of the maximum gradient of the streamwise velocity in the vertical direction $\left(\frac{\partial u}{\partial y}\right)$ within the shear layer at locations downstream of the separation point. Figure 4-22 shows the locations of $\left(\frac{\partial u}{\partial y}\right)_{\text {MAX }}$ for the two cases of yaw angle $=0^{\circ}$ and yaw angle $=90^{\circ}$. The figure shows that the shear layer centre connects the upstream and downstream lips of the cavity in an almost straight line, in both cases. This is not unexpected, considering that both cases studied here represent the deep cavity flow regime, in which the shear layer is known to bridge the cavity opening. This behaviour also indicates that the difference in the velocity profiles at the upper edge of the cavity in the two cases is not due to the effect of the shear layer being drawn into the cavity (as in the case of shallow cavities).

The analysis by Ashcroft and Zhang can be used to study the differences in the thickness of the shear layer [23]. They have studied the growth of the shear layer past the separation point for rectangular cavities with depth to length ratios in the range of $0.25 \leq h / D \leq 0.5$, exposed to turbulent boundary layers at $2.2 \times 10^{5} \leq \operatorname{Re} \leq 5.6 \times 10^{5}$ 
based on cavity length (D), by investigating the variations of vorticity thickness (Equation 2-11). The vorticity thickness growth rate $\left(d \delta_{\omega} / d x\right)$ between 0.114 and 0.3 have been reported for nominally two-dimensional rectangular cavities [23]-[26].

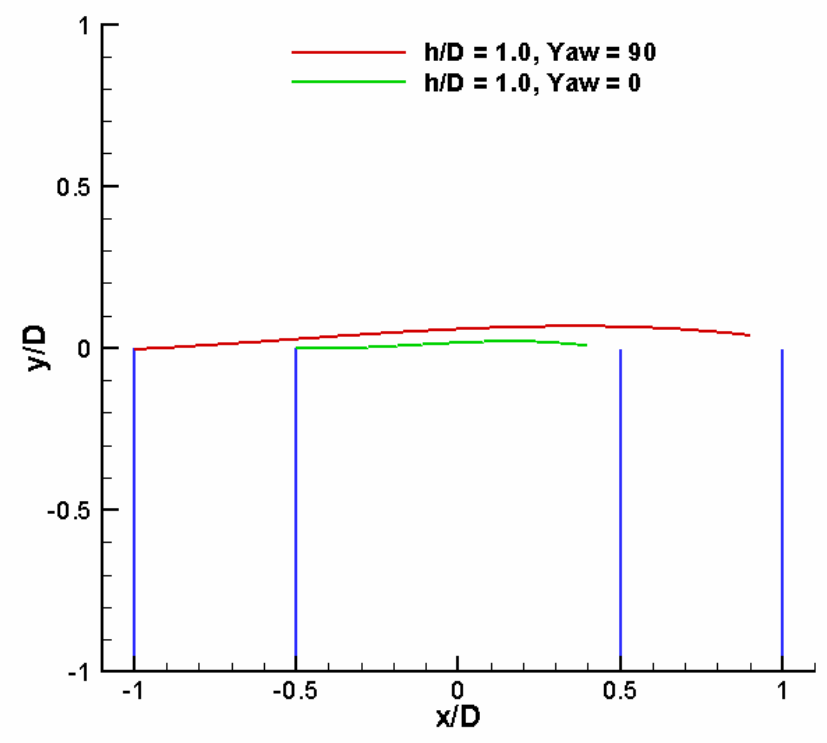

Figure 4-22 The location of shear layer centre $(h / D=1.0)$

Figure 4-23 shows variations of vorticity thickness along the shear layer, for the cavities at yaw angle $=0^{\circ}$ and yaw angle $=90^{\circ} . x^{\prime}$ is measured from the upstream cavity lip. It should be mentioned that since $\mathrm{D}$ is the length of minor axis of the cavity, in the case of $90^{\circ}$ yaw angle, the streamwise length of the cavity is $2 \mathrm{D}$. The figure shows that the shear layer grows uniformly past the separation point for both cases, regardless of the yaw angle, until it reaches the vicinity of the downstream lip of the cavity, where its thickness starts to decrease. This behaviour is similar to that reported by Ashcroft and Zhang [23] for the rectangular cavity. A comparison of $\delta_{\omega}$ at the centre of the cavity $(\mathrm{x} / \mathrm{D}=0)$ for the two cases $\left(x^{\prime} / \mathrm{D}=0.5\right.$ for $0^{\circ}$ yaw angle and $x^{\prime} / \mathrm{D}=1.0$ for $90^{\circ}$ yaw angle $)$ indicates that the shear layer has grown thicker in the case of yaw angle $=90^{\circ}$, due to larger distance from the separation point. The thicker shear layer in this case has resulted in larger velocities in the cavity, as shown in Table 4-6, which compares velocities at $y / D=0$ and $\mathrm{y} / \mathrm{D}=-0.2$ for the two cases. 


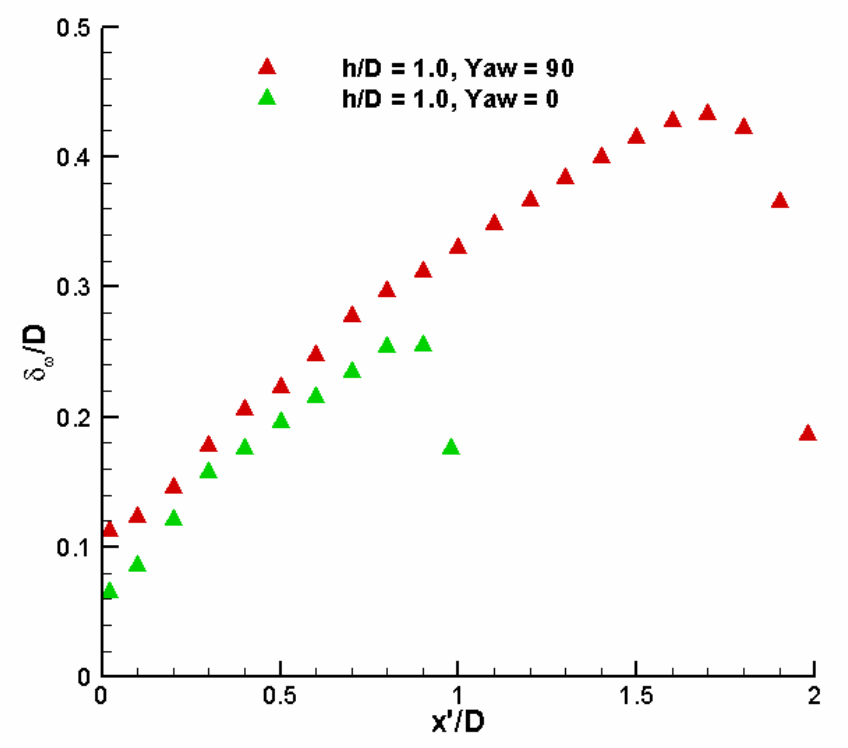

Figure 4-23 Shear layer thickness

Table 4-6 $\bar{u} / \mathrm{U}_{\text {inf }}$ for two yaw angles at two different locations $(\mathrm{h} / \mathrm{D}=1.0)$

\begin{tabular}{|c|c|c|}
\hline Yaw angle & $\bar{u} / \mathrm{U}_{\mathrm{inf}}(\mathrm{y} / \mathrm{D}=0)$ & $\bar{u} / \mathrm{U}_{\mathrm{inf}}(\mathrm{y} / \mathrm{D}=-0.2)$ \\
\hline $0^{\circ}$ & 0.308 & -0.003 \\
\hline $90^{\circ}$ & 0.348 & 0.602 \\
\hline
\end{tabular}

Based on the above-mentioned arguments, the velocity at the upper edge of the cavity $(y / D=0)$ at the streamwise location of $x / D=0$ (centre of the cavity) has been chosen as reference velocity $\left(U_{r e f}\right)$ to compare the profiles obtained form experiments and numerical simulations (Figure 4-24). The use of this reference velocity accounts for the quantitative differences in the velocity profiles due to different boundary layer thicknesses, and highlights the different profiles shapes at different yaw angles, due to differences in the thickness of the shear layer. It should be noted that due to the optical restriction caused by the boundary layer plate, $y / D=-0.2$ is the highest position at which velocity data are available from the PIV experiments. Therefore, velocity at the edge of the cavity has been extrapolated using the relationship between the velocities at $y / D=$ 
-0.2 and $y / D=0$ in the results of the numerical simulations, and the velocity at $y / D=-0.2$ in the experimental data.

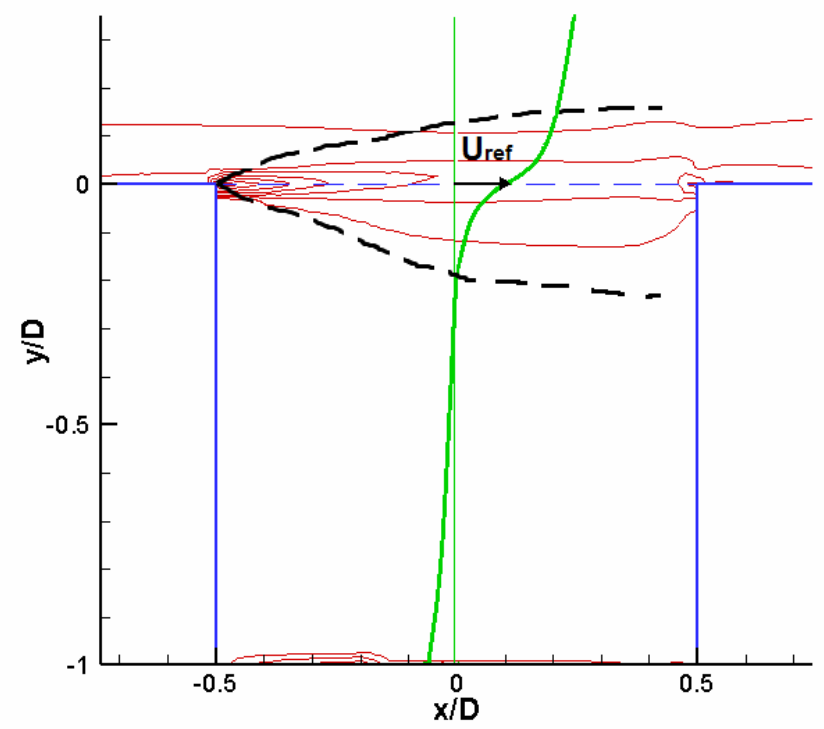

Figure 4-24 Schematic figure of velocity profile at $\mathbf{x} / \mathbf{D}=\mathbf{0}$ (green line), contours of $\frac{\partial u}{\partial y}$ (red lines), shear layer (black dashed line), cavity walls (blue lines), and $U_{\text {ref }}$ (black vector)

\subsection{Detecting Vortex Core Position}

Several methods can be used to detect the location of the vortex core. Three approaches for identification of the vortex core have been compared in Figure 4-25, which shows the results of PIV measurements in a plane located at $\mathrm{z} / \mathrm{D}=0$, for a cavity with $\mathrm{h} / \mathrm{D}=1.0$ at the yaw angle of $90^{\circ}$.

Figure 4-25 shows, (a) sectional streamlines generated based on the in-plane velocity vector components, (b) contours of the out of plane vorticity z-component $\omega_{z}=\partial v / \partial x-\partial u / \partial y,(\mathrm{c})$ contours of the streamfunction $(\psi)$ and $(\mathrm{d})$ contours of $\mathrm{Q}$ criterion. A comparison of Figure 4-25-(a) and (b) shows that the first two methods indicate a similar location for the vortex core. However, Contours of $\psi$ show a slightly different location for the vortex core. This is due two reasons: one reason is the approximation in 
numerical integration of the velocity field, which has to be carried out to determine $\psi$. The second is the fact that $\psi$ is a parameter defined for potential, two-dimensional flow. The flow under investigation does not have any of these two properties, therefore, $\psi$ cannot be expected provide an accurate representation of the flow pattern.

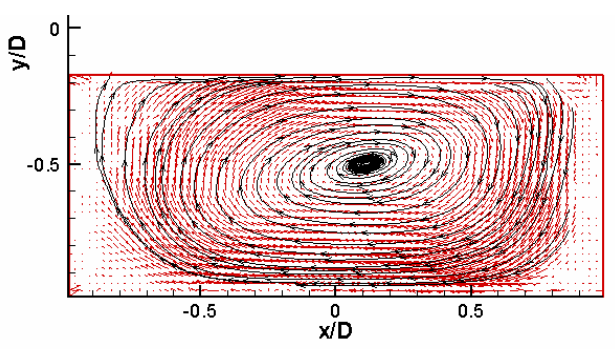

(a) Stream trace

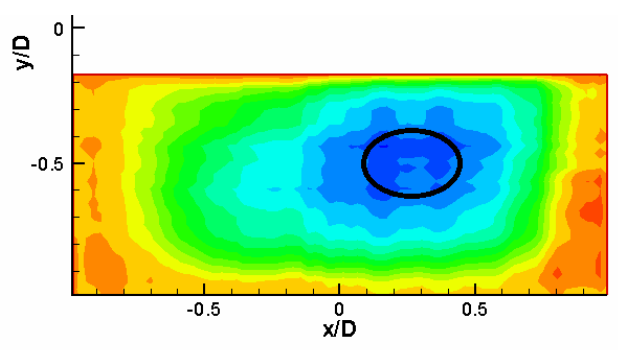

(c) Stream function contours $(\psi)$

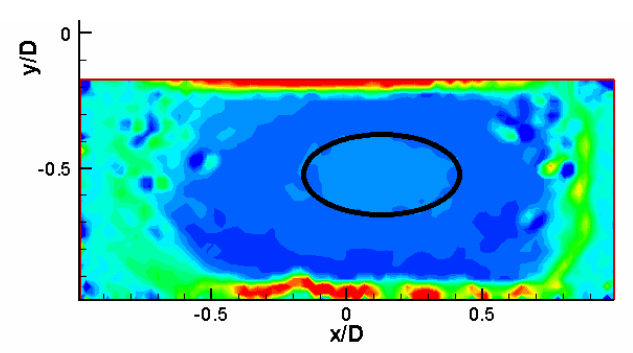

(b) Vorticity contours $\left(\omega_{z}\right)$

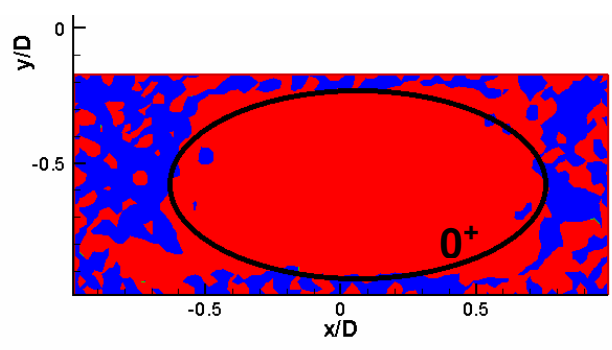

(d) Q criterion

Figure 4-25 Different methods to find vortex core location inside the cavity $\left(\mathrm{h} / \mathrm{D}=1.0 \&\right.$ yaw $\left.=90^{\circ}\right)$

As mentioned in Chapter 2, the Q criterion is a method for confirming the existence of a vortex in a flow field, where other factors, such as the shear layer, also contribute to vorticity [11]. This method differentiates vortices from other contributors to vorticity by subtracting the strain rate from vorticity (Equation 2-9).

Although this method is useful for identifying a vortex in the flow field, it can only specify the boundary of the vortex, which corresponds to the $Q=0^{+}$iso-value (i.e. the smallest positive iso-value), and not the location of the vortex core. As observed in Figure 4-25-(d), the vortex boundary, indicated by the $Q=0^{+}$iso-value, covers a large part of the measurement plane and so does not provide any information about the location of the vortex core. 
It should be noted that that iso-values of pressure can also provide a representation of vortex cores in the flow field. However, the use of this criterion is limited to the results of numerical simulations in the present study, since the experiments do not include pressure measurements in the cavity volume. Of the first three methods, the sectional streamlines based on the velocity vectors (Figure 4-25-(a)) have been found to provide the most detailed results, which compare very well with the vortex cores determined based on pressure iso-surfaces. Therefore, sectional streamlines have been used to determine the location of the vortex core in the following chapter.

\subsection{Proper Orthogonal Decomposition}

The Proper Orthogonal Decomposition (POD) method projects a data ensemble comprised of $N$ samples of a vector field variable $u_{i}\left(x_{i}, t\right)$, onto $N$ orthogonal spatial modes $\varphi^{n}\left(x_{i}\right)$. The POD modes are energetically optimal, so that a reconstruction of the data ensemble using the first $M \leq N$ POD modes contains more energy that any other reconstruction involving a similar number of modes obtained on any other basis. Holmes et al. [116] have shown that the POD modes can be obtained by finding the eigenvectors of the following problem:

$$
\int\left\langle u_{i}\left(x_{i}, t\right) \cdot u_{i}\left(x_{i}{ }^{\prime}, t\right)\right\rangle \varphi^{n}\left(x_{i}{ }^{\prime}\right) d x_{i}{ }^{\prime}=\lambda^{n} \varphi^{n}\left(x_{i}\right)
$$

The $\langle$.$\rangle operator represents spatial autocorrelation. The discretization and solution$ procedure for this problem can be found in the works of Smith et al. [117] and Shlens [118]. The eigenvalues $\lambda^{n}$ in Equation 4-15 represent the energy content of each mode. Each spatial POD mode is accompanied by a time-varying amplitude coefficient, which can be obtained by projecting the data ensemble onto each mode, using the following equation:

$$
a^{n}(t)=\int u_{i}\left(x_{i}, t\right) \cdot \varphi^{n}\left(x_{i}^{\prime}\right) d x_{i}
$$

The combination of the POD modes and time-varying amplitude coefficients can be used to reconstruct the original data ensemble, using the following equation: 


$$
u_{i}^{*}\left(x_{i}, t\right)=\sum_{n=1}^{M} a^{n}(t) \varphi^{n}\left(x_{i}\right)
$$

In Equation 4-17, $u_{i}^{*}\left(x_{i}, t\right)$ is an approximate reconstruction of the original data ensemble, using $M \leq N$ POD modes, also known as a low-dimensional representation.

Due to its unique features, including orthonormality and optimal energy content of the modes, POD analysis is an ideal means for filtering out the noise in a complex data ensemble, to reveal the underlying dynamic behaviour. In Chapter 5 of the present study, this method has been utilized to analyze the oscillations of the vortex in the cavity based on the results of the PIV velocity field measurements by calculating phase averaged velocity fields.

\subsection{Summary}

In this chapter, the wind tunnel, model design process and specifications, measurement equipments and the experimental setup were described in detail. Also, the boundary layer flow, measurement uncertainties, and data analysis methods were explained comprehensively.

Table 4-7 shows the different cases investigated through the experimental measurements and the numerical simulations. As mentioned in Chapter 3, for the numerical simulations, the dimensions and boundary layer specifications of Hering's experiments were replicated. However, in the present experiments, due to the practical limitations explained in section 4.4, it was not possible to maintain some of the experiment parameters at similar values. The details and comparison of the experiment parameters are presented in Table 4-8. The parameters have been expressed in non dimensional form where applicable, to facilitate the comparison.

The results of the experiments conducted using the equipment and methods described in this chapter are presented and discussed in the following chapter. 
Table 4-7 List of numerical simulation and experimental measurement cases

\begin{tabular}{|l|l|l|l|}
\hline \multicolumn{2}{|l|}{ Method } & h/D & Yaw angle (degrees) \\
\hline \multicolumn{2}{|l|}{ Numerical simulation } & $\begin{array}{l}0.1,0.2,0.35, \\
0.5,0.7, \text { and } 1.0\end{array}$ & $0,15,30,45,60$, and 90 \\
\hline \multirow{2}{*}{$\begin{array}{l}\text { PIV } \\
\text { measurement }\end{array}$} & Inside the cavity & $0.5,0.7$, and 1.0 & $0,15,30,45,60$, and 90 \\
\cline { 2 - 4 } & Downstream of the cavity & 0.5 and 0.7 & 0,45, and 90 \\
\hline Flow visualization & 1.0 & 0,45, and 90 \\
\hline
\end{tabular}

Table 4-8 Comparison of the parameters of present and Hering's experiments [58]

\begin{tabular}{|l|c|c|}
\hline Parameter & Present experiments & Hering (2006) \\
\hline Cavity minor axis $(\mathrm{D})$ & $72.5 \mathrm{~mm}$ & $53.75 \mathrm{~mm}$ \\
\hline Freestream velocity $\left(\mathrm{U}_{\mathrm{inf}}\right)$ & $18.3 \mathrm{~m} / \mathrm{s}$ & $27 \mathrm{~m} / \mathrm{s}$ \\
\hline Re $_{\mathrm{D}}$ & $8.7 \times 10^{4}$ & $9.1 \times 10^{4}$ \\
\hline Boundary layer thickness $(\delta / \mathrm{D})$ & 0.60 & 1.08 \\
\hline Displacement thickness $\left(\delta^{*} / \mathrm{D}\right)$ & 0.06 & 0.15 \\
\hline Momentum thickness $(\theta / \mathrm{D})$ & 0.04 & 0.11 \\
\hline Shape factor $(\mathrm{H})$ & 1.5 & 1.4 \\
\hline Skin friction coefficient $\left(C_{f}\right)$ & 0.0035 & 0.0026 \\
\hline Friction velocity $\left(u_{*} / \mathrm{U}_{\mathrm{inf}}\right)$ & 0.040 & 0.036 \\
\hline
\end{tabular}




\section{Results and Discussion}

\subsection{Introduction}

In this chapter, the results of the experimental measurements and numerical simulation of the flow in and around the elliptical cavity, which were described in the previous chapters, will be presented and analyzed. The main objectives of this chapter are as follows:

- To establish a comprehensive representation of the flow structure in the elliptical cavity volume, in order to complement previous studies of the flow structure based on surface pressure measurements

- To analyze the effect of depth on the flow structure inside and around elliptical cavities, in order to identify the flow regimes and features affected by depth and to determine similarities and differences with cavities with other planform shapes.

- To analyze the effect of yaw angle on the flow structure inside and around elliptical cavities with various depths, and to determine the flow structure and mechanisms associated with the increased drag forces associated with strongly asymmetric flows.

- To investigate the effects of wall curvature on the flow structure in elliptical cavities at various combinations of cavity depth and yaw angle, and through comparison with the flows associated with rectangular cavities.

- To analyze the flow in the wake of the cavity, in order to investigate the features of the flow in the wake that are related to the cavity flow, in terms of velocity and vorticity

- To study the dynamic behaviour of the flow in the cavity, including fluctuating velocity components and vortex oscillations 
- To identify the underlying mechanisms from which the above-mentioned effects originate, to compare them with previous findings for elliptical cavities and other cavity planforms where applicable, and to highlight the flow features unique to elliptical cavities.

Throughout the analysis of the results, a combination of the data obtained from numerical simulations, experimental measurements and visualizations of the flow, and the results of the experiments by Hering [58] will be used. In order to use this combination, quantitative and qualitative agreement between these three sources of data must be established, to ensure that the data support the observations mutually. This comparison will be presented in the following section, and prior to analysis of the results.

Hering and Savory [6] have categorized the flow regimes in an elliptical cavity with a major to minor axis ratio of (2:1), similar to the one studied herein, for various depths and yaw angles. This categorization, which is based on surface pressure measurements, is shown in Figure 5-1. The categories of flow regimes specified in this figure will be used as a guideline for selecting the combinations of cavity depth and yaw angle for which the flow will be analyzed in detail. Based on this categorization, the analysis of the results will be carried out for three following major flow regimes, which are highlighted by rectangles in Figure 5-1:

- Symmetric flow regimes: These flow regimes are associated with shallow, intermediate-depth, and deep cavities at $0^{\circ}$ yaw angle. The analysis of results for these regimes will be carried out for $\mathrm{h} / \mathrm{D}$ ratios of $0.1,0.2,0.35,0.5,0.7$, and 1.0 (blue region).

- Nominally two-dimensional flow regime: This flow regime is associated with cavities at the yaw angle of $90^{\circ}$. The analysis will be carried out for three depths $(\mathrm{h} / \mathrm{D}=0.1,0.5$, and 1.0), which represent the shallow, intermediate depth, and deep cavities (green region).

- Asymmetric flow regimes: These flow regimes are divided into two subcategories: The weakly asymmetric regime, which is associated with cavities with 
yaw angles up to $30^{\circ}$, and the strongly asymmetric regime, which is associated with cavities with yaw angles of $45^{\circ}$ and $60^{\circ}$. The analysis of results will be carried out for three depths $(\mathrm{h} / \mathrm{D}=0.1,0.5$, and 1.0$)$, which represent the shallow, intermediate depth and deep cavities (red region).

The analysis of the results includes position and development of the shear layer, position of the vortices in the cavity, surface pressure distribution and drag, flow downstream of the cavity, and dynamics of the flow. Qualitative and quantitative comparisons will be carried out with previously published results for elliptical cavities, as well as those of other comparable flows, where available.

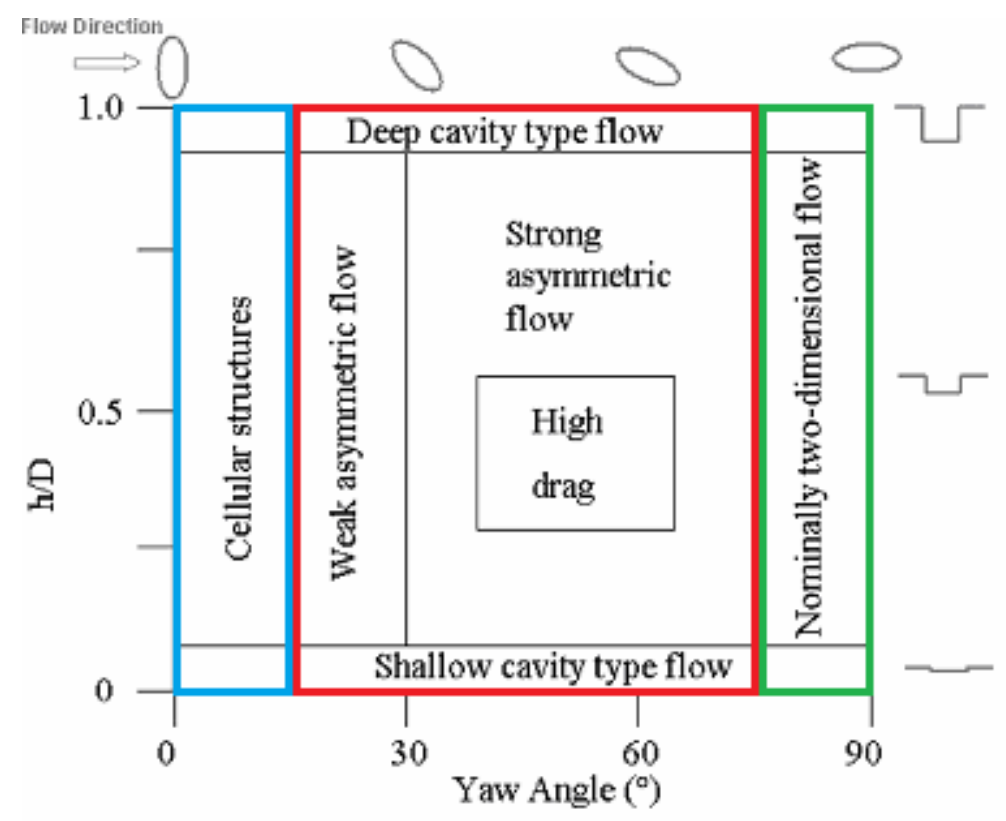

Figure 5-1 Different flow regimes of yawed elliptical cavity (adapted from ref. [6]). Symmetric, asymmetric and nominally two-dimensional regimes are highlighted in blue, red, and green, respectively.

\subsection{Comparative Evaluation of the Experimental and Numerical Results}

As mentioned earlier, a combination of the results of PIV velocity field measurements and numerical simulations will be used to analyze the results in this chapter. The 
experimental and numerical results of the present study will be complemented by the results of the pressure measurement experiments by Hering [58], where applicable. In order to study the flow based on a combination of these sources of data, quantitative and qualitative agreement among them should be established. In the following paragraphs, various comparisons between the results of the present experiments and numerical simulations, and the experiments by Hering will be carried out to verify the agreement among the results.

\subsubsection{Comparison of PIV Experiments and Numerical Simulations}

\subsubsection{Mean and turbulent velocity profiles}

In this section, profiles of the streamwise component of mean velocity $(\bar{u})$ and the streamwise component of turbulent kinetic energy, expressed by $\overline{u^{\prime} u^{\prime}}$, obtained from the PIV experiments and the numerical simulations, will be compared to examine the agreement between the two sources of data. The comparison includes profiles at selected locations inside the cavity, for representative combinations of $\mathrm{h} / \mathrm{D}$ and yaw angle.

The process of choosing an appropriate reference velocity $\left(U_{\text {ref }}\right)$ for normalizing the results is an important aspect of this comparison. This process illustrates the mechanism by which momentum and velocity is transferred from the boundary layer to the cavity. The details concerning the choice of reference velocity were given in section 4.10.

The cases of the cavity with $\mathrm{h} / \mathrm{D}=1.0$ at yaw angles of 0,45 and 90 degrees have been used as representative cases to examine the agreement between the numerical and experimental profiles. The reason for selecting this cavity depth is that the measurement area is the largest compared to the cases with other depths and yaw angles, therefore, more comparable data points were available. These three selected yaw angles represent the shortest and longest distances from the separation point, as well as an intermediate case.

Figure 5-2 shows the profiles of the streamwise component of mean velocity $(\bar{u})$ and the streamwise component of turbulent kinetic energy, expressed by $\overline{u^{\prime} u^{\prime}}$, based on numerical 
and experimental results, for the cavity with $\mathrm{h} / \mathrm{D}=1.0$ at $90^{\circ}$ yaw angle. The profiles have been extracted along the vertical (y) direction, for three streamwise locations, corresponding to $\mathrm{x} / \mathrm{D}=-0.5,0$, and 0.5 , on the centreline of the cavity. The results show similar trends for the experimental and numerical results, in the profiles of $\bar{u}$ and $\overline{u^{\prime} u^{\prime}}$.

Figure 5-3 and Figure 5-4 show the profiles of $\bar{u}$ and $\overline{u^{\prime} u^{\prime}}$, based on numerical and experimental results, for the cavity with $\mathrm{h} / \mathrm{D}=1.0$ at the yaw angles of $45^{\circ}$ and $0^{\circ}$ respectively. The profiles have been extracted along the vertical (y) direction, for three streamwise locations, corresponding to $\mathrm{x} / \mathrm{D}=-0.25,0$, and 0.25 , on the centreline of the cavity. Again, similar trends are observed for the experimental and numerical results.

A comparison of the velocity profiles at yaw angles $=0^{\circ}$ and $90^{\circ}$ verifies the trend shown in Figure 4-21 Velocity profile comparison for two different yaw angles at two different locations for the experimental data. Due to the larger thickness of the shear layer in the case of yaw angle $=90^{\circ}$ due to increased growth over a larger distance from the separation point, the profiles show milder gradients, and flow with larger velocity being entrained into the cavity, compared to the case of yaw angle $=0^{\circ}$.

The error bars in these figures represent the uncertainty based on the combination of random statistical errors and the error in velocity measurement using the PIV technique. Estimation of the uncertainties has been described in section 4.9. 


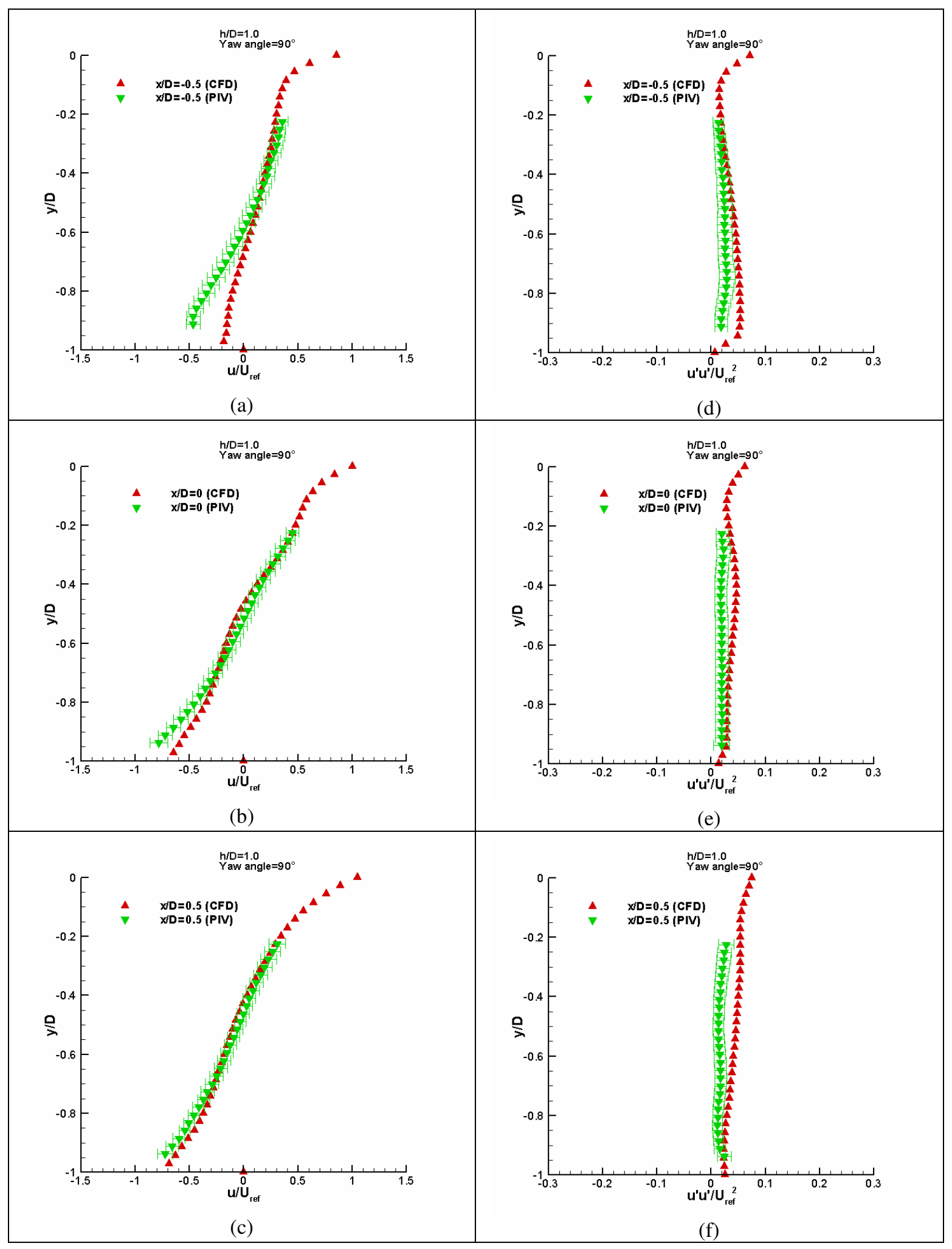

Figure 5-2 Numerical and experimental results comparison of $\bar{u}$ and $\overline{u^{\prime} u^{\prime}}$ profiles for $\mathbf{h} / \mathrm{D}=1.0$ at $90^{\circ}$ yaw angle 


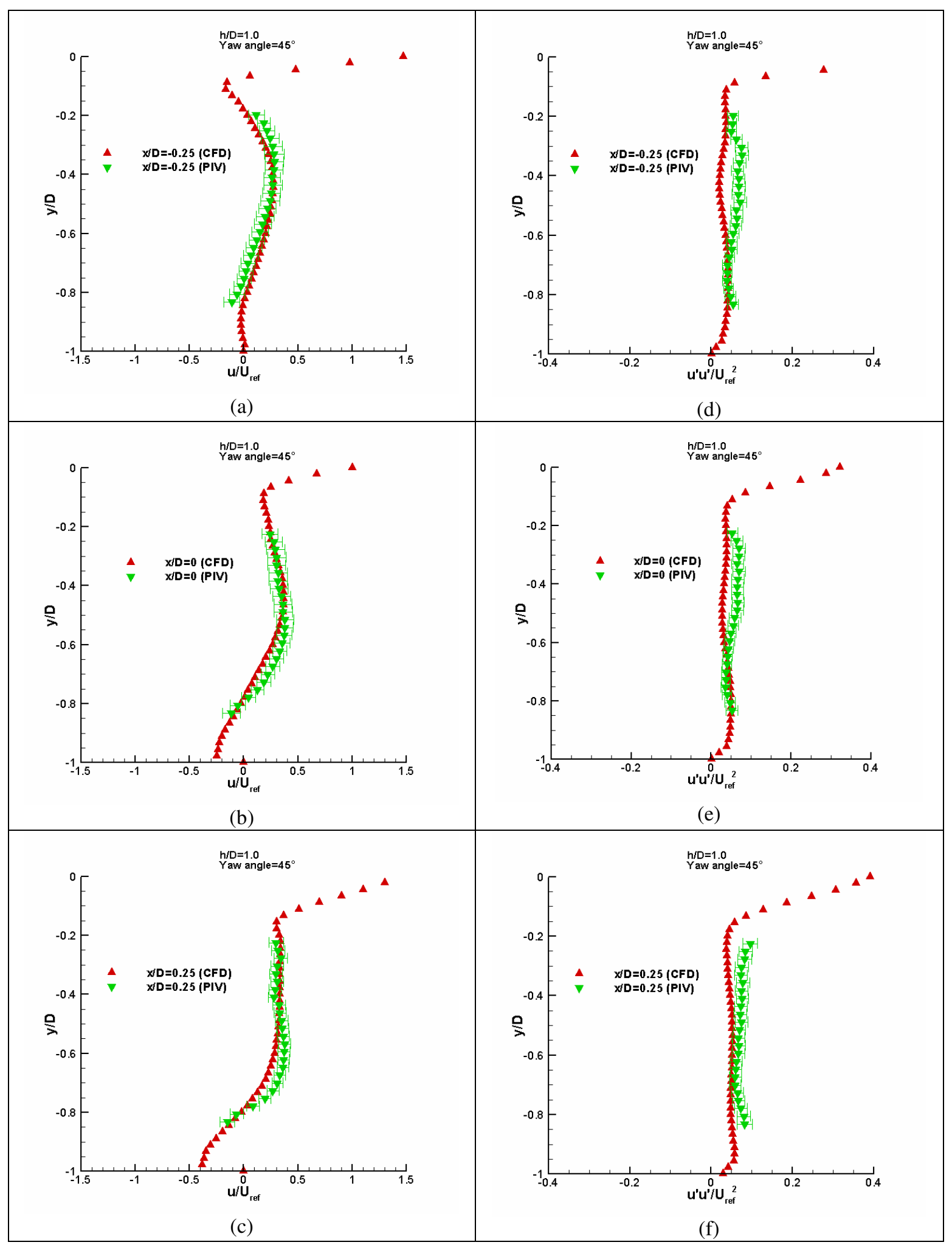

Figure 5-3 Numerical and experimental results comparison of $\bar{u}$ and $\overline{u^{\prime} u^{\prime}}$ profiles for $\mathbf{h} / \mathrm{D}=1.0$ at $45^{\circ}$ yaw angle 


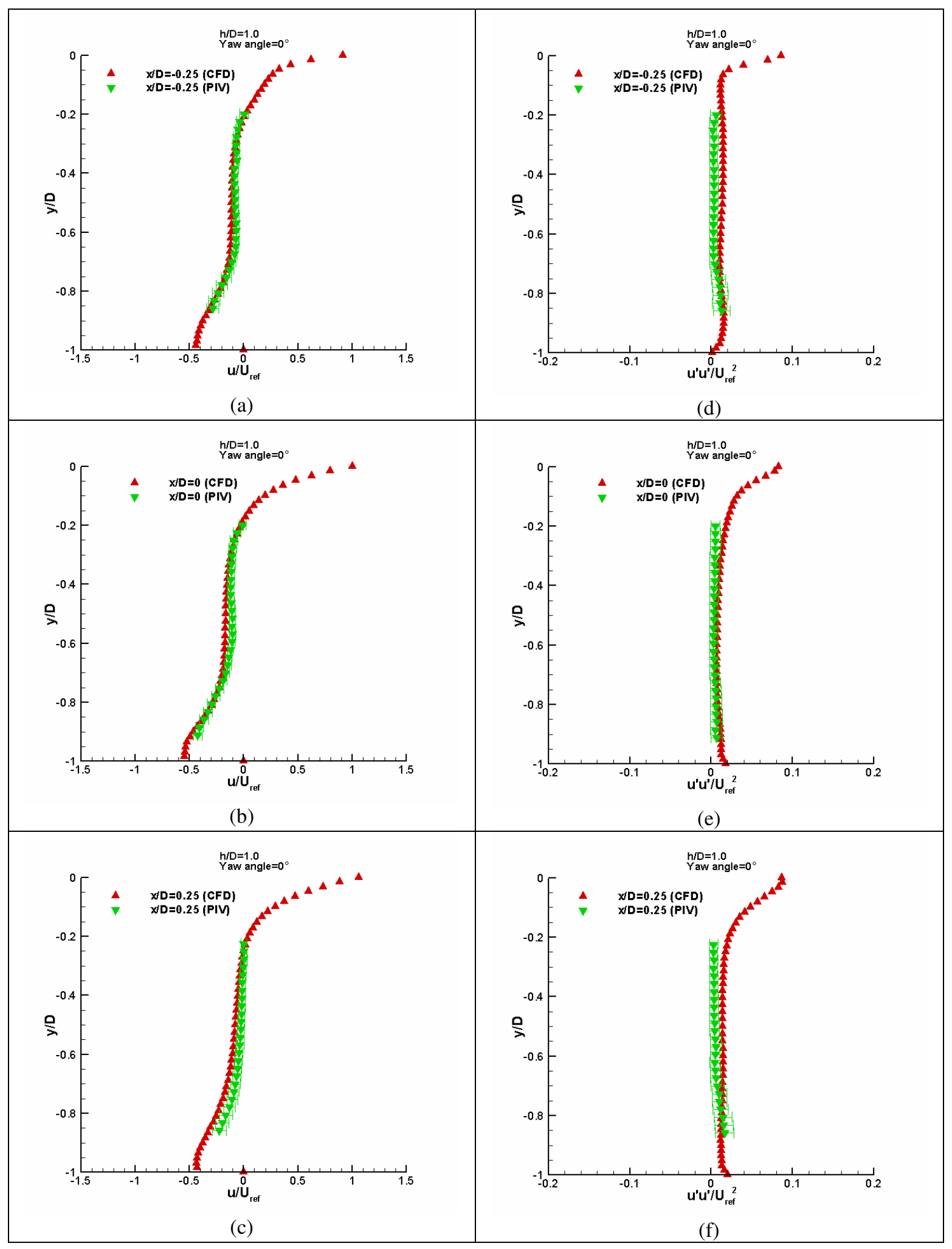

Figure 5-4 Numerical and experimental results comparison of $\bar{u}$ and $\overline{u^{\prime} u^{\prime}}$ profiles for $\mathbf{h} / \mathrm{D}=1.0$ at $0^{\circ}$ yaw angle 


\subsubsection{Vortex core positions}

The profiles presented above provide detailed quantitative information about the distribution of flow parameters at selected locations. However, it is difficult to infer the flow structure inside the cavity using the limited number of local profiles. Therefore, the location of the vortex core in the three-dimensional volume of the cavity, determined based on the experimental and numerical results, will be used in as tool to describe the flow structure in this chapter. In the case of the experimental results, the location of the vortex in the three-dimensional volume of the cavity has been reconstructed by detecting the vortex core based on the results of the PIV measurements in the horizontal and vertical planes listed in the previous chapter. In the case of the numerical results, planes similar to those involved in the PIV measurements have been used to extract data from the three-dimensional volume of the cavity. A complete discussion of the methods for determining the location of the vortex core has been presented in section 4.11.

Considering the agreement between the experimental and numerical velocity profiles presented in the previous section, the positions of the vortex cores are also expected to be in agreement. Nevertheless, the agreement between the experimental and numerical vortex core positions has been verified quantitatively, by calculating the average distance between the vortex cores obtained based on experimental and numerical results, for selected cases.

Table 5-1 shows the average distance between vortex cores obtained using the experimental and numerical results $(\bar{\Delta})$, as a quantitative criterion for assessing the agreement of the two data sets. The values in the table are normalized using the cavity minor axis (D). Based on the values presented in the table, it can be observed that the average distance between the vortex cores is $0.07 \mathrm{D}$.

The uncertainty in the position of vortex core has also been calculated based on the standard deviation of the vortex core positions determined from instantaneous PIV snapshots. The calculation has been carried out for a number of combinations of measurement plane, cavity depth, and yaw angle, with various levels of velocity fluctuations $\left(u_{r m s}\right.$ and $\left.v_{r m s}\right)$, which represent cases with various levels of vortex 
oscillations, as shown later in section 5.3.5. The results indicate a maximum uncertainty of $\pm 0.05 \mathrm{D}$ in the horizontal ( $\mathrm{x}$ ) direction and $\pm 0.02 \mathrm{D}$ in the vertical (y) direction. A comparison of the maximum uncertainty with the average difference between vortex core positions shown in Table 5-1 suggests that the vortex cores obtained based on experimental and numerical results are in reasonable agreement, and can be used in combination to describe the flow structure in the cavity.

Table 5-1 Average distance between vortex cores (CFD \& PIV results)

\begin{tabular}{|c|c|c|}
\hline$h / D$ & Yaw angle $\left({ }^{\circ}\right)$ & $\bar{\Delta} / D$ \\
\hline 1 & 0 & 0.06 \\
\hline 1 & 15 & 0.06 \\
\hline 1 & 30 & 0.08 \\
\hline 1 & 45 & 0.07 \\
\hline 1 & 60 & 0.11 \\
\hline 1 & 90 & 0.09 \\
\hline 0.5 & 0 & 0.06 \\
\hline 0.5 & 15 & 0.10 \\
\hline 0.5 & 30 & 0.05 \\
\hline 0.5 & 45 & 0.05 \\
\hline 0.5 & 60 & 0.05 \\
\hline 0.5 & 90 & 0.07 \\
\hline \multicolumn{2}{|c|}{ Average value } & 0.07 \\
\hline
\end{tabular}

\subsubsection{Relationship between Pressure Distribution and Vortex Core Position}

Haigermoser et al. [24] have shown the correspondence between low-pressure regions in the flow field and the location of vortex core using PIV measurements for a nominally two-dimensional rectangular cavity exposed to a thick turbulent upstream boundary layer flow. They have also shown the correspondence between the high-pressure regions and the locations of shear layer impingement onto the cavity walls. This correspondence for the elliptical cavity involved in the present study will be demonstrated in the following paragraphs. This will be done by investigating the relationship between the vortex core positions obtained based on the present PIV velocity field measurements and numerical 
simulations, the pressure distributions obtained from the present numerical simulations, and the pressure distributions reported by Hering [58].

The investigation will also evaluate the agreement between the results of the present experiments and numerical simulations, and the experiments of Hering [58]. Establishing this agreement will make it possible to use all three data sources in combination to describe the cavity flow in various $\mathrm{h} / \mathrm{D}$ ratios and yaw angles in this chapter. Throughout the process of validation of the numerical simulation methodology in section 3.7, quantitative agreement between the results of the present numerical simulations and the experiments by Hering [58] has been demonstrated. The quantitative comparison, which involves distribution of pressure coefficients along the centreline of the cavity base for selected combinations of cavity depth and yaw angle, indicates a maximum difference of $5 \%$ between pressure coefficients. To complement the quantitative comparison, position of the vortex core, and surface pressure distributions based on the present numerical experimental results, and the experimental results by Hering [58] will be compared in the following paragraphs, for three representative cases, with different flow regimes associated with various combinations of cavity depth and yaw angle.

It should be noted that the objective of the following comparison is only to further confirm agreement of the results from the three independent sources, and not to describe the flow structure. Considering this objective, only a brief overview of the flow structure has been given for each case. The detailed description of the cavity flow structure for these cases will be presented later in this chapter.

The viewing angle for each case has been chosen individually to show the threedimensional features of the particular vortex core.

The first example is the cavity with $\mathrm{h} / \mathrm{D}=1.0$ at yaw angle $=0^{\circ}$, which is an example of the "deep cavity" flow regime (Figure 5-1). Figure 5-5 shows the vortex core position in the cavity volume based on the present numerical and experimental results in part (a), as well as contours of pressure coefficient in and around the cavity based on the present numerical results (part b), and those of Hering's experiments (part c). Figure 5-5-(a) shows a three-dimensional vortex core. The centre part of the vortex core is close to the 
upstream wall of the cavity between the angular positions of $60^{\circ}$ and $300^{\circ}$, at an approximate depth between $\mathrm{y} / \mathrm{D}=-0.25$ and -0.5 . The pressure coefficient distributions shown in Figure 5-5-(b) and (c) illustrate a low-pressure area on the sidewall of the cavity, which covers the same range of angular position and depth as the vortex core. The contours shown in parts (b) and (c) are plotted using the same scale and number of levels, and can also be used to verify the quantitative agreement between the two sets of results.

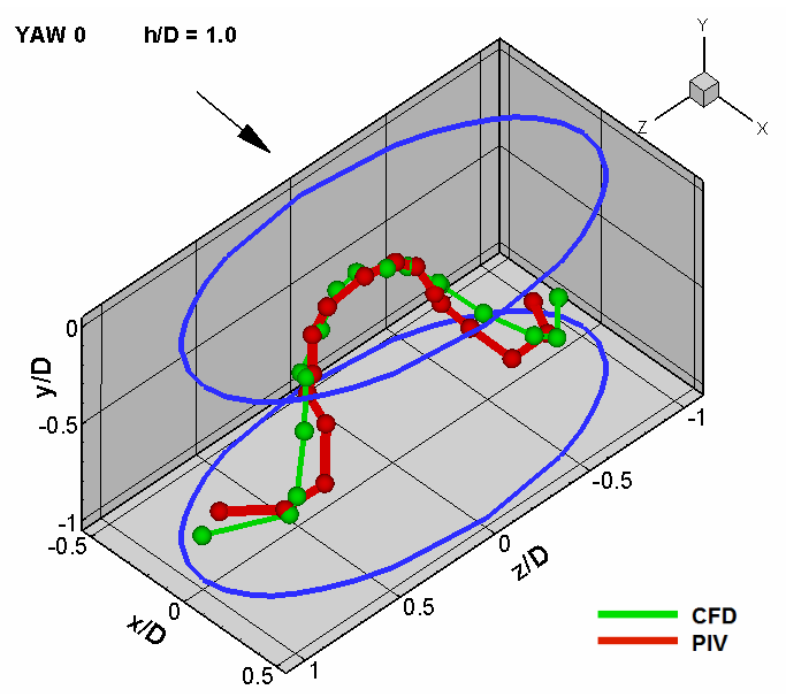

(a)

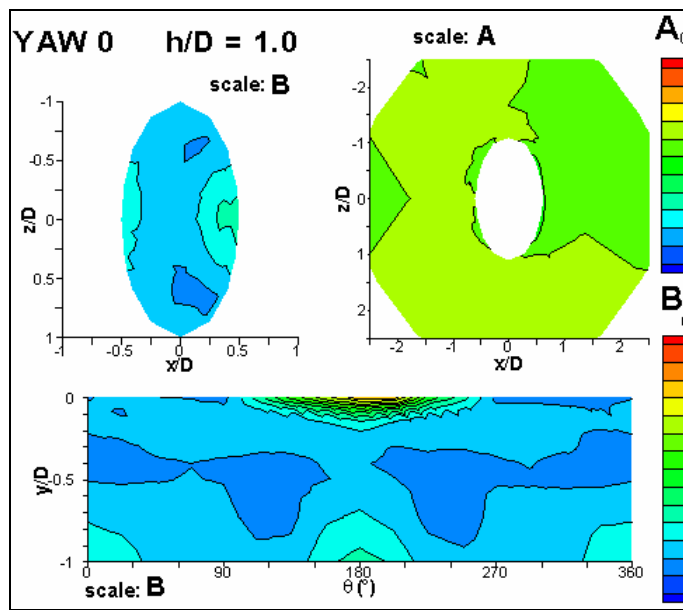

(b)

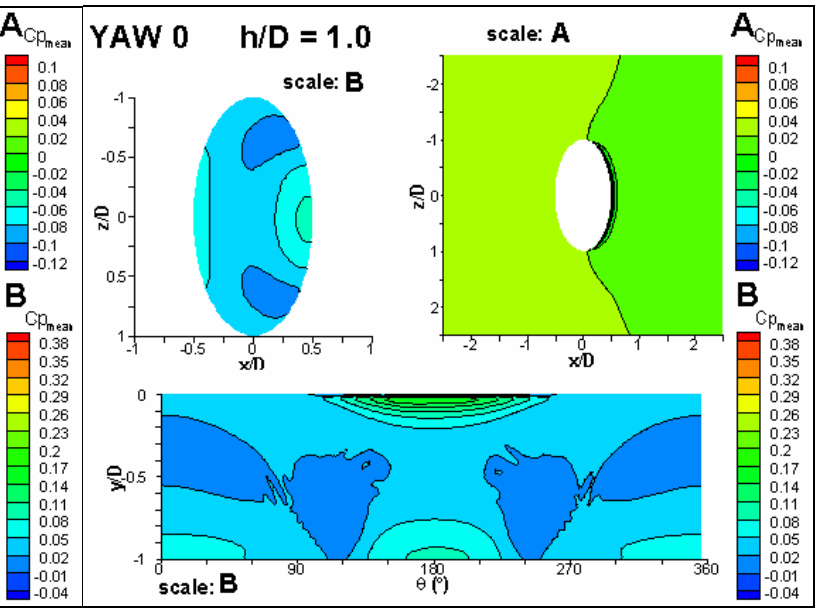

(c)

Figure 5-5 Vortex core position (a) and pressure coefficient contours on cavity base, ground plane, and side walls for $\mathrm{h} / \mathrm{D}=\mathbf{1 . 0}$, yaw angle $=0^{\circ}$ from reference [58] (b) and numerical simulations (c) 
The two extreme ends of the vortex core are located lower than the centre part $(y / D<-$ $0.5)$, and are close to the sidewalls at angular positions of $120^{\circ}$ and $240^{\circ}$. The pressure distributions shown in parts (b) and (c) indicate low pressure regions at the same positions. The extreme ends of the vortex core also approach the floor of the cavity at two locations ( $\mathrm{x} / \mathrm{D}$ between 0 and 0.25 , and $\mathrm{z} / \mathrm{D}= \pm 0.75$, approximately). Again, the pressure distributions show low-pressure areas at the same locations.

Figure 5-6 shows the results for the cavity with $\mathrm{h} / \mathrm{D}=0.5$ at yaw angle $=45^{\circ}$. This case is an example of the "strongly asymmetric" regime (Figure 5-1). Figure 5-6-(a) indicates a curved vortex core, in which the centre part is closer to the cavity floor. This corresponds to a low-pressure region on the cavity base in parts (b) and (c). According to Figure 5-6(a), the upstream end of the vortex is bound by the cavity sidewall at an angular position of $60^{\circ}$, and a depth between $-0.2 \mathrm{D}$ and $-0.3 \mathrm{D}$, with a corresponding low-pressure region at the same location in parts (b) and (c). The downstream end of the vortex core approaches the upper edge of the cavity at an angular position between $180^{\circ}$ and $270^{\circ}$, and leaves the cavity volume to form a trailing vortex. Again, the pressure distributions shown in parts (b) and (c) show a low-pressure area corresponding to the same location.

Figure 5-7 (a) shows the vortex core for the case of $\mathrm{h} / \mathrm{D}=0.5$ and yaw angle $=90^{\circ}$, which represents the regime categorized as "nominally two-dimensional". The results of the numerical simulations indicate a primary, and a smaller secondary vortex in the cavity. The secondary vortex is very close to the cavity base, and cannot be observed in results of PIV measurements due to reflections from the cavity base, except for the mid-plane, where the core was slightly higher. No trace of this weak vortex can be observed in the pressure distributions. The primary vortex core is relatively straight in the spanwise direction and curved slightly towards the cavity floor at its centre. The centre of the vortex is located at a streamwise position of $x / D=0.3$, which corresponds with a lowpressure area at the same location in the pressure distributions in Figure 5-7-(b) and (c). Both the vortex core position and the low pressure regions in the pressure distributions on the cavity sidewall indicate that the two ends of the vortex approach the side walls of the cavity at angular position slightly above $90^{\circ}$ and slightly below $270^{\circ}$. Based on these 
observations, the correspondence between the location of the vortex core and the lowpressure regions on the cavity walls can be confirmed for this case.

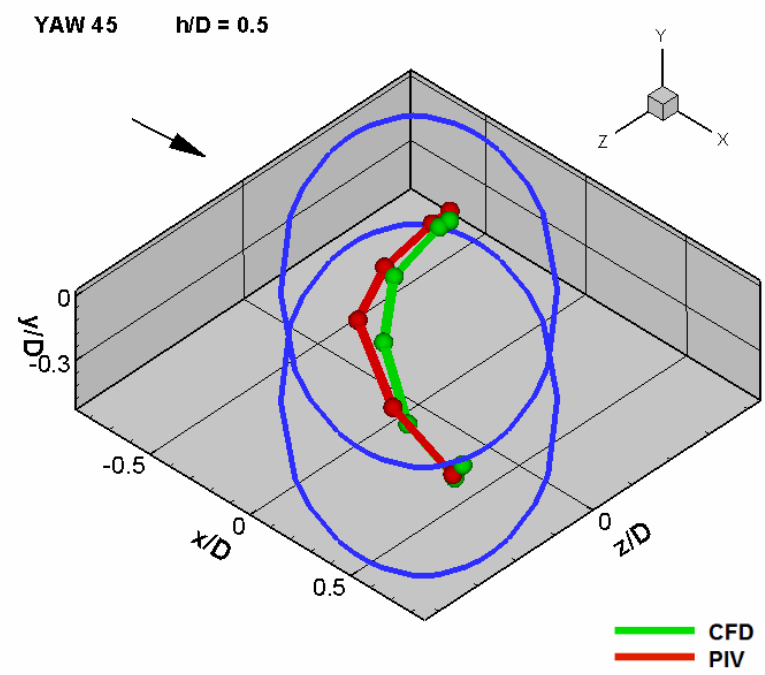

(a)
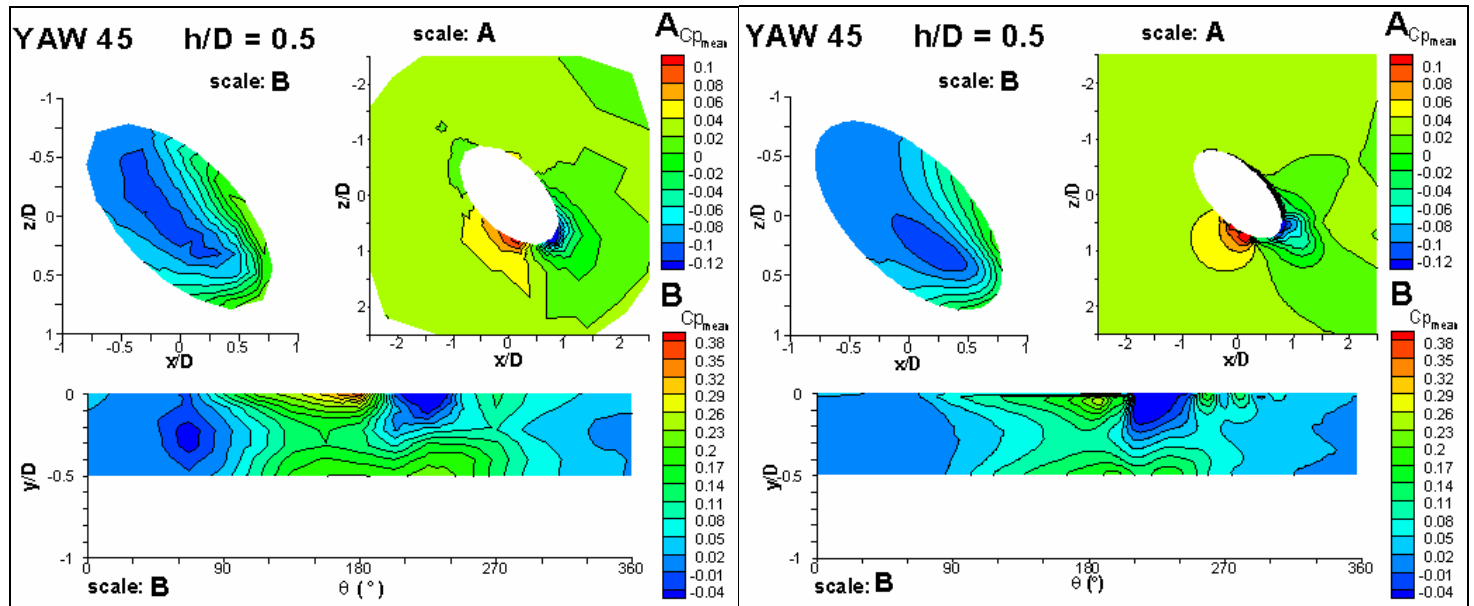

(b)

(c)

Figure 5-6 Vortex core position (a) and pressure coefficient contours on cavity base, ground plane, and side walls for $\mathrm{h} / \mathrm{D}=\mathbf{0 . 5}$, yaw angle $=45^{\circ}$ from reference [58] (b) and numerical simulations (c)

Based on the above-mentioned observations, it can be concluded that the direct correspondence between the location of the vortex core in the cavity volume and the low pressure areas, which was shown by Haigermoser et al. [24] for a two-dimensional rectangular cavity, continues to exist in the case of the three-dimensional elliptical cavity 
studied herein. The results also confirm the agreement between the results of the PIV measurements, the numerical simulations, and the pressure measurement experiments by Hering [58].

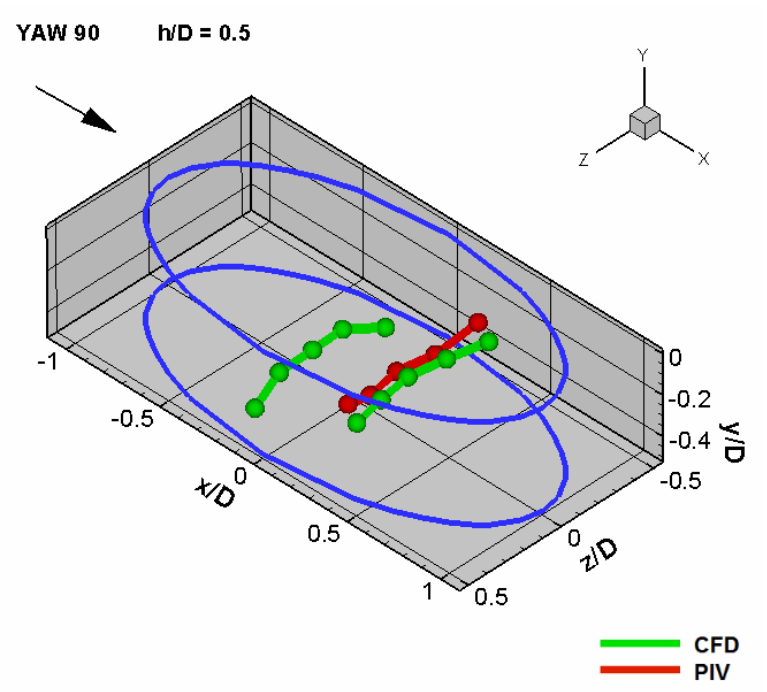

(a)

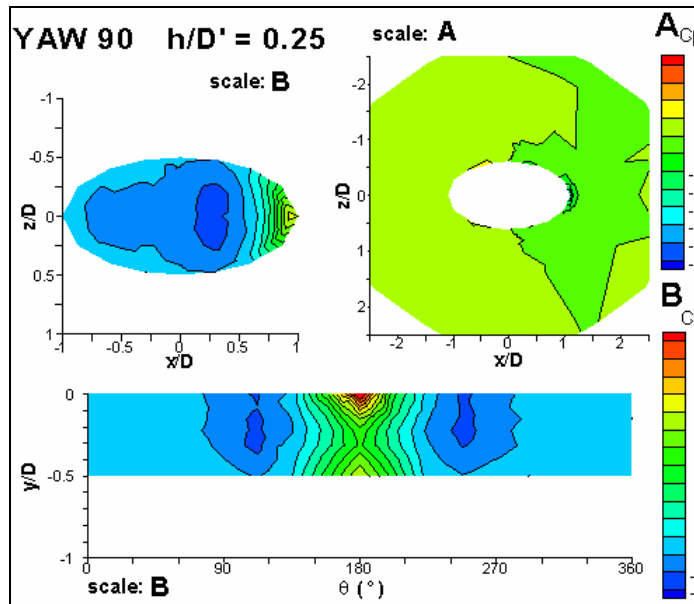

(b)

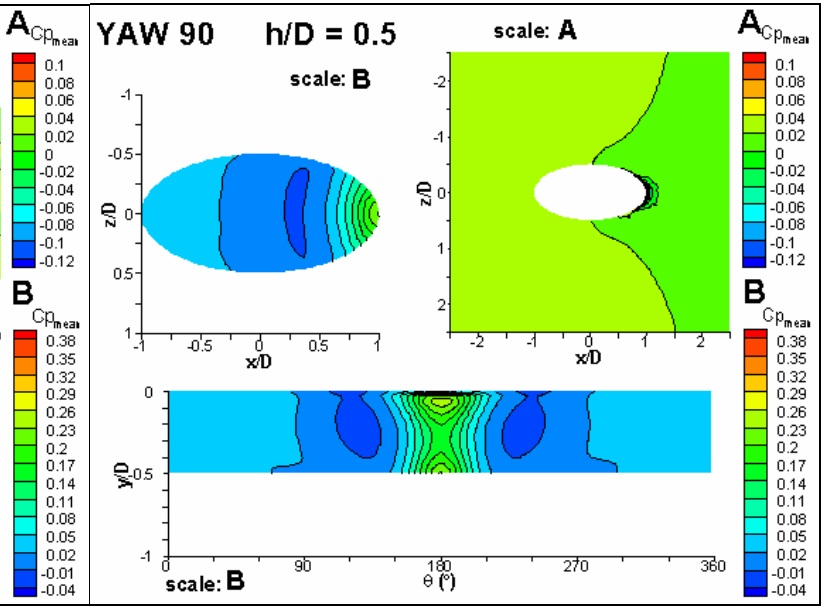

(c)

Figure 5-7 Vortex core position (a) and pressure coefficient contours on cavity base, ground plane, and side walls for $\mathrm{h} / \mathrm{D}=0.5$, yaw angle $=90^{\circ}$ from reference [58] (b) and numerical simulations (c)

In this section, quantitative agreement between the mean and turbulent velocity profiles based on the experimental and numerical results of the present study was shown in section 5.2.1. This was followed by demonstration of the direct correspondence between the location of vortex in the cavity and pressure distributions on cavity walls, based on 
the experimental and numerical results of the present study, and the results reported by Hering [58], which also confirmed the agreement between the results of the three sources. The comparisons presented in this section, together with the quantitative agreement between pressure distributions, shown in section 3.7, confirms the results from the three sources are in mutual agreement, and a combination of them can be used to analyze the flow in the following sections.

\subsection{Symmetric Flow Regimes}

Symmetric flow regimes are associated with the cavity at $0^{\circ}$ yaw angle. The main feature of the flow in these regimes is symmetry of the flow structure based on mean flow properties, with respect to the mid-plane of the cavity $(\mathrm{z} / \mathrm{D}=0)$. As suggested by Figure $5-1$, and indicated by the results presented in the following paragraphs, when the depth of the cavity changes, significant changes in the flow structures occur, while the flow maintains its symmetry. These changes lead to three distinct flow regimes: the "deep cavity" flow regime, associated with the cavity with $\mathrm{h} / \mathrm{D}=1.0$, the "intermediate depth" flow regime, associated with cavities with h/D between 0.35 and 0.5 , and "shallowtransitional cavity" flow regime, associated with the cavity with $\mathrm{h} / \mathrm{D}=0.1$ and 0.2 . Each of these three regimes have distinct features, which will discussed in detail in the following paragraphs based on various aspects of the flow, including shear layer behaviour, the three-dimensional flow structure, drag, flow downstream of the cavity, and the dynamic behaviour. Comparisons with previously published studies will be included where comparable results are available.

\subsubsection{Behaviour of the Shear Layer}

As previously discussed in detail in Chapter 2, the flow in a cavity exposed to an upstream boundary layer is dominated by the interaction of the shear layer separated from the upstream edge of the cavity and the solid boundaries of the cavity. Therefore, the behaviour of the separated shear layer can provide valuable insight into the flow structure. In this section, two parameters have been used for studying the behaviour of the shear layer. These parameters are the thickness of the shear layer and the position of the 
shear layer centre. The method for determining these two parameters has been described in section 4.10 .

Figure 5-8 shows variations of the thickness of the shear layer shear along the upper edge of the cavity $(y / D=0)$ in the cavity mid-plane $(z / D=0)$, determined based on the results of the numerical simulations, for cavities with $\mathrm{h} / \mathrm{D}$ ratios between 0.1 and 1.0 , at $0^{\circ}$ yaw angle. The figure indicates that the shear layer grows following an almost linear trend, after separation from the upstream edge, with a growth rate that is similar of all $\mathrm{h} / \mathrm{D}$ ratios. The results of the studies by Ashcroft and Zhang [23], Haigermoser et al. [24], Murray et al. [25], and Ukeiley and Murray [26] indicate that the shear layer separated from the upstream edge of nominally two-dimensional rectangular cavities exposed to turbulent boundary layers displays a similar behaviour, in which growth rate is linear and independent of the geometric proportions of the cavities such as relative depth and length.

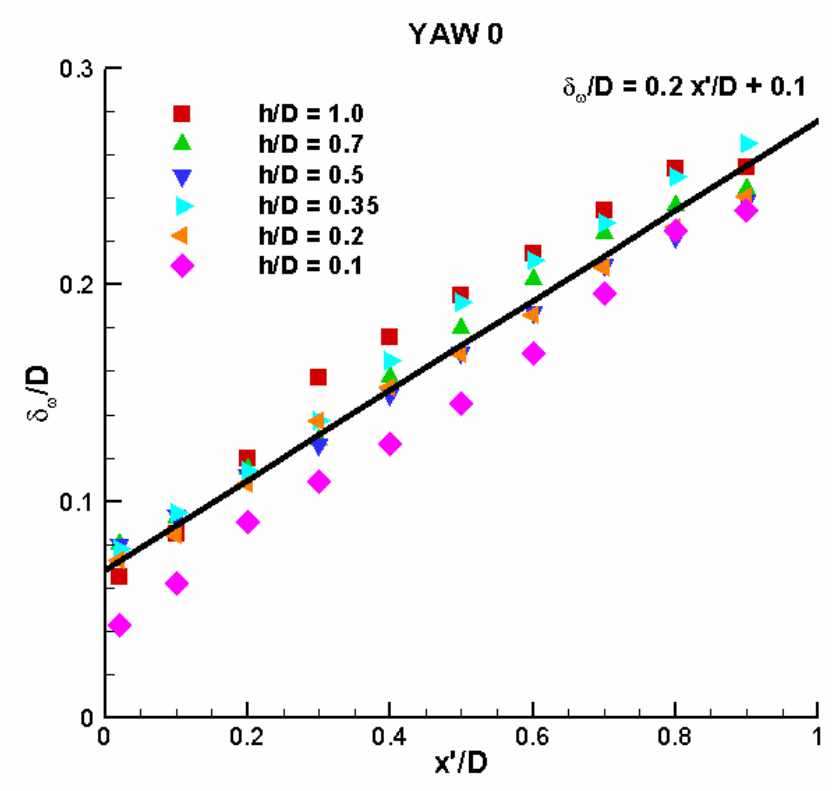

Figure 5-8 Shear layer thickness at $0^{\circ}$ yaw angle

The quantitative value of shear layer growth rate for the results shown in Figure 5-8 is $d \delta_{\omega} / d x=0.2$. For nominally two-dimensional rectangular cavities, values between 0.114 and 0.3 have been reported [23]-[26]. The value obtained based on the present 
results within the above-mentioned range, and is particularly close to the value of $d \delta_{\omega} / d x=0.19$ reported by Haigermoser et al. [24] for open rectangular cavities with h/D $=0.25$ exposed to a turbulent approaching flow with $\operatorname{Re}_{D}=O\left(10^{4}\right)$.

YAW 0

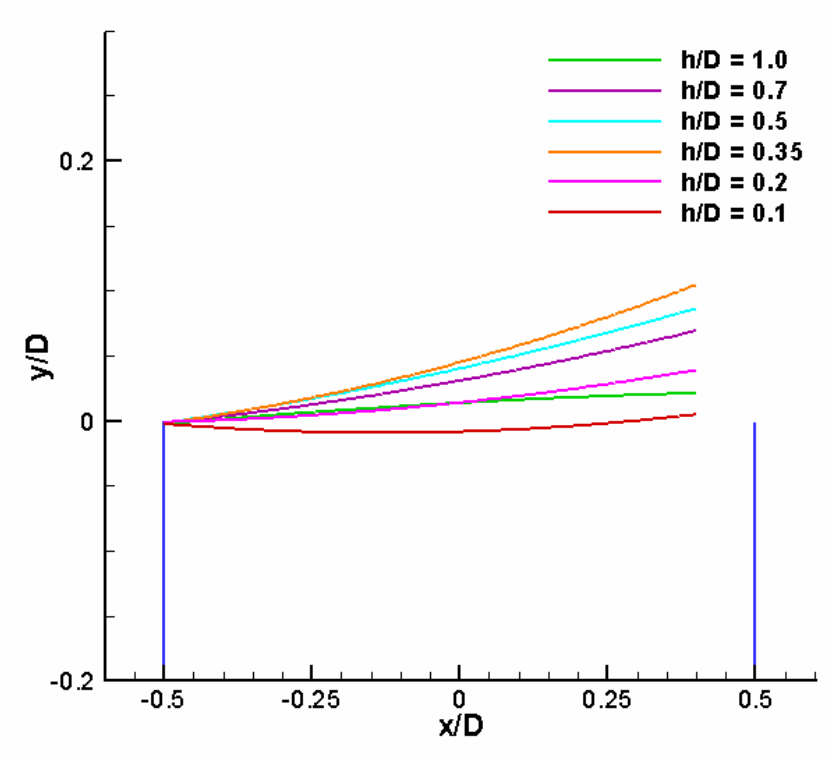

Figure 5-9 The location of shear layer centre at $0^{\circ}$ yaw angle

Figure 5-9 shows the position of the centre of the shear layer relative to the upper edge of the cavity in the cavity mid-plane $(\mathrm{z} / \mathrm{D}=0)$, determined based on the results of the numerical simulations, for cavities with $\mathrm{h} / \mathrm{D}$ ratios between 0.1 and 1.0 , at $0^{\circ}$ yaw angle.

An examination of the shear layer centre positions at $h / D=1.0$ indicates that the shear layer at this depth to width ratio extends over the upper edge of the cavity without considerable deflection into or out of the cavity volume. The behaviour of the shear layer in this case similar that of a nominally two-dimensional cavity in the "open" regime associated with deep cavities [15]. Since the shear layer thickness grows linearly downstream of the separation point (Figure 5-9), it can be concluded that an increasingly larger part of it is entrained further downstream of the separation point. This trend continues up to the vicinity of the downstream edge of the cavity, where the shear layer impinges upon the downstream wall. 
The shear layer centre positions at $\mathrm{h} / \mathrm{D}=0.1$ indicate that the shear layer centre is deflected into the cavity volume downstream of the separation point. Considering the growth of the shear layer downstream of the separation point (Figure 5-8), one can expect the shear layer to impinge upon, or approach the cavity base. The behaviour of the shear layer in this case resembles that of nominally two-dimensional rectangular cavity in the "closed" or "transitional-closed" regimes associated with shallow cavities.

Between the above-mentioned extremes of cavity depth, a significant change in the behaviour of the shear layer centre locations can be observed for the $\mathrm{h} / \mathrm{D}$ ratios of 0.7 , $0.5,0.35$, and 0.2. For these intermediate depths, the shear layer centre appears to be deflected upwards in the downstream half of the upper edge of the cavity. This deflection increases as the $\mathrm{h} / \mathrm{D}$ ratio decreases from 0.7 to 0.35 , and then decreases again at $\mathrm{h} / \mathrm{D}=$ 0.2. This behaviour of the shear layer centre locations cannot be directly associated with the typical "open" or "closed" regimes of deep and shallow cavities, but may be indicative of a different flow structure.

The behaviour of the shear layer depth will be related to the flow structure in the cavity in the following section, in which the flow structure for cavities with various $h / D$ at $0^{\circ}$ yaw angle will be investigated in detail.

\subsubsection{Flow Structure}

In the following paragraphs, a combination of the results of the PIV velocity field measurement, numerical simulations, and flow visualizations carried out in the present study, and the experiments by Hering [58] are used to identify the flow structure in elliptical cavities at $0^{\circ}$ yaw angle. Comparisons with previously published results are included where available.

\subsubsection{Deep cavity $(\mathrm{h} / \mathrm{D}=1 \mathrm{1.0})$}

Figure 5-5-(a) shows the vortex core position for the cavity with $\mathrm{h} / \mathrm{D}=1.0$. A more detailed image of the flow pattern in two vertical planes, located at $\mathrm{z} / \mathrm{D}=0$ and $\mathrm{z} / \mathrm{D}=0.7$, is shown in Figure 5-10. While the streamtraces shown in Figure 5-10 both indicate a 
flow pattern similar to that of a deep, nominally two-dimensional rectangular cavity containing a single captive vortex [15], considerable variation in the position and orientation of the vortex core is found to exist across the span, due to three-dimensional effects.

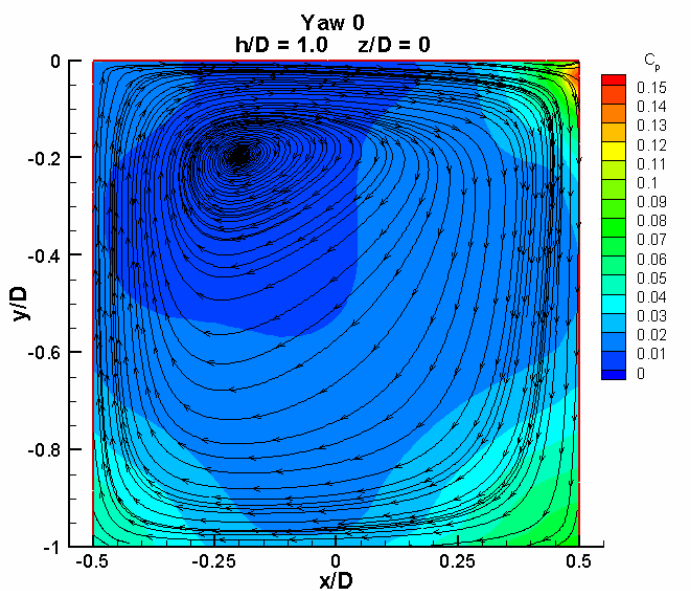

(a)

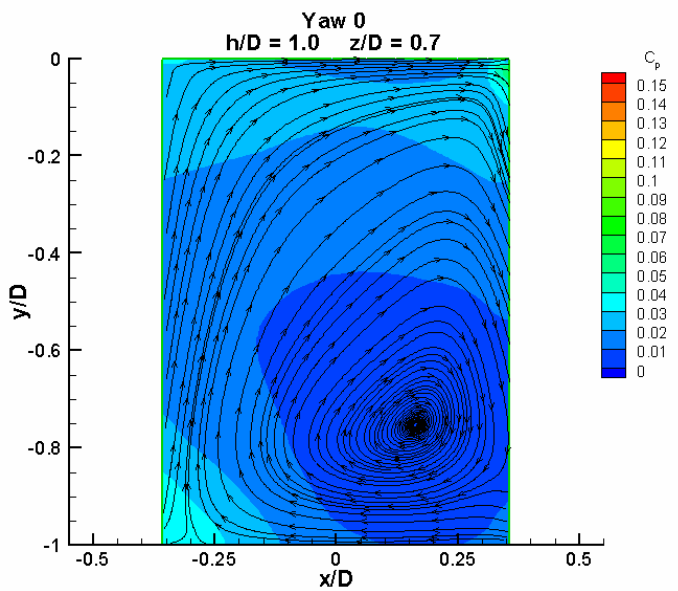

(b)

Figure 5-10 Streamlines and $C_{p}$ contours for cavity with $h / D=1.0$ at $0^{\circ}$ yaw angle at $\mathrm{z} / \mathrm{D}=0$ (a) and $\mathrm{z} / \mathrm{D}=0.7(\mathrm{~b})$

At the centre of the cavity $(\mathrm{z} / \mathrm{D}=0)$, the vortex core is located at a smaller depth, and closer to the upstream wall at the centre of the cavity. With increasing distance from the mid-plane, the core extends slightly downstream and downwards towards the base of the cavity, so that the two ends of the vortex core are bounded by the upstream wall, near the floor of the cavity. The downstream deflection of the vortex core with increasing distance from the centreline may be because of the curvature of the upstream wall in the downstream direction, which leads to formation of the recirculation region further downstream, at spanwise positions farther from the centreline. The pressure coefficient contours based on numerical simulations shown in Figure 5-5-(c), are in accordance with the above-mentioned flow structure. As described in section 5.2.2, the low-pressure contours are associated with the locations at which the vortex core approaches the cavity walls. Figure 5-5-(c) shows a low-pressure region on the upper half of the cavity sidewall, between the angular positions of $315^{\circ}$ and $45^{\circ}$, which corresponds with the centre part of the vortex core. The two low pressure regions on the cavity floor 
correspond with the parts of the vortex core that approach the cavity floor. At the locations where the two ends of the vortex core reach the sidewalls, two low pressure regions can be observed on the lower half of the sidewalls, at the angular positions of $120^{\circ}$ and $240^{\circ}$. A symmetric high pressure region can be observed at the upper edge of the downstream wall of the cavity, around the angular position of $180^{\circ}$. This region corresponds to the location where the shear layer impinges upon the downstream cavity wall after bridging the cavity, as shown clearly in Figure 5-10-(a).

Figure 5-11 shows an instantaneous visualization of the flow inside the cavity with $\mathrm{h} / \mathrm{D}=$ 1.0 at $0^{\circ}$ yaw angle, obtained using the Helium bubble technique. This figure shows a captive vortex inside the cavity. The vortex core is located in the upstream part of the cavity, and close to the upper edge. The location of the vortex core in this image corresponds to the location of the vortex core in the mid-plane $(\mathrm{z} / \mathrm{D}=0)$, shown in Figure 5-10-(a).

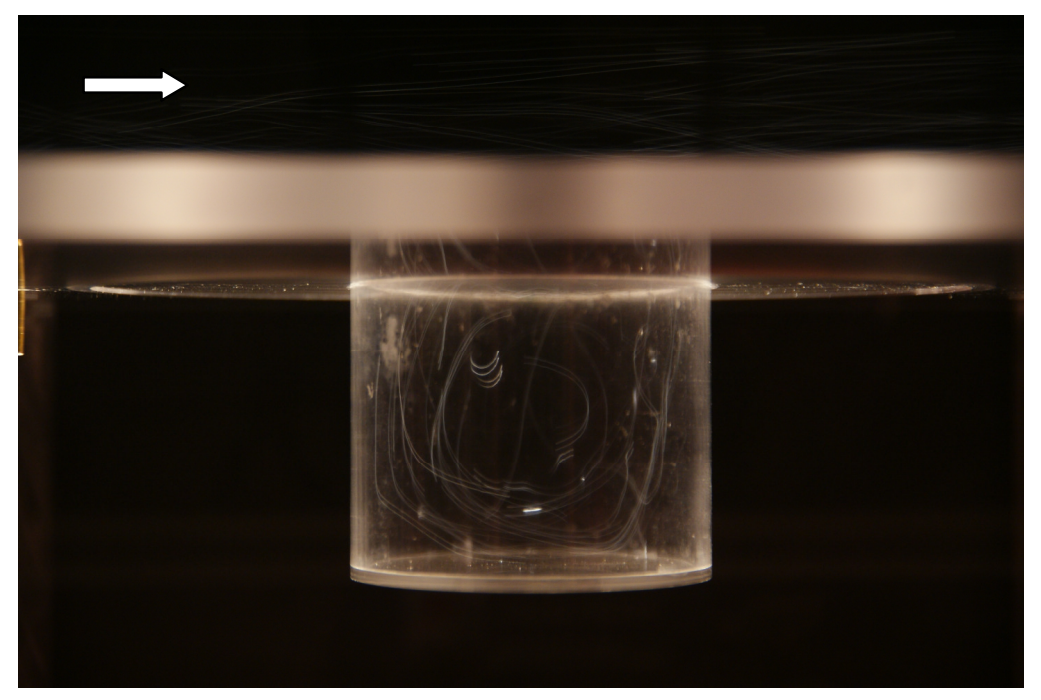

Figure 5-11 Instantaneous flow visualization image obtained using the Helium bubble technique, for the cavity with $\mathrm{h} / \mathrm{D}=1.0$ at yaw angle $=0^{\circ}$

The pressure coefficient contours based on the experiments by Hering [58] are shown in Figure 5-5-(b). A comparison between Figure 5-5-(b) and Figure 5-5-(c) indicates similar patterns in pressure distributions. Considering the correspondence between surface 
pressure distributions and position of the vortex (discussed in section 5.2.2), it can be concluded that the vortex structure identified based on the present results is similar to that of Hering's experiments.

\subsubsection{Intermediate-depth cavities $(0.35 \leq h / D \leq 0.7)$}

As expected from the previously-mentioned change in the location of the shear layer position from the case of $h / D=1.0$ to those of $h / D=0.7,0.5$, and 0.35 , the vortex core positions and surface pressure distributions also indicate a flow structure that is significantly different from that of the deep cavity.

The vortex cores for the cases of $\mathrm{h} / \mathrm{D}=0.7,0.5$, and 0.35, shown in Figure 5-12-(a), (b) and (c), illustrate a W-shaped structure which resembles the cellular structure introduced in Chapter 2 based on the work of Maull and East [16]. The three-dimensional features of the vortex are clearly visible in this figure, which show the symmetric, W-shaped vortex inside the cavity. The centre portion of this vortex is closer to the downstream wall of the cavity and the two extreme ends of the vortex stretch towards the upstream wall of the cavity. Due to the shape of the vortex, it impinges upon the downstream wall of the cavity at two locations, which are symmetrically located at the two sides of the centre of the vortex core. This behaviour is confirmed by the pressure contours shown in Figure 5-12-(d), (e) and (f), which show a two-cell low-pressure region at the two sides of the angular position of $180^{\circ}$, on the downstream wall. The high pressure regions associated with the location of shear layer impingement on the downstream wall also show a cellular behaviour, in which two symmetric high pressure regions can be observed at the approximate angular positions of $135^{\circ}$ and $225^{\circ}$, based on pressure distribution on the

downstream wall. This is in contrast with the cavity with $\mathrm{h} / \mathrm{D}=1.0$, for which one high pressure region due to shear layer impingement was observed around the centreline of the downstream wall. This difference can also be observed in Figure 5-13 which shows a high pressure region at $\mathrm{z} / \mathrm{D}=0.7$. 


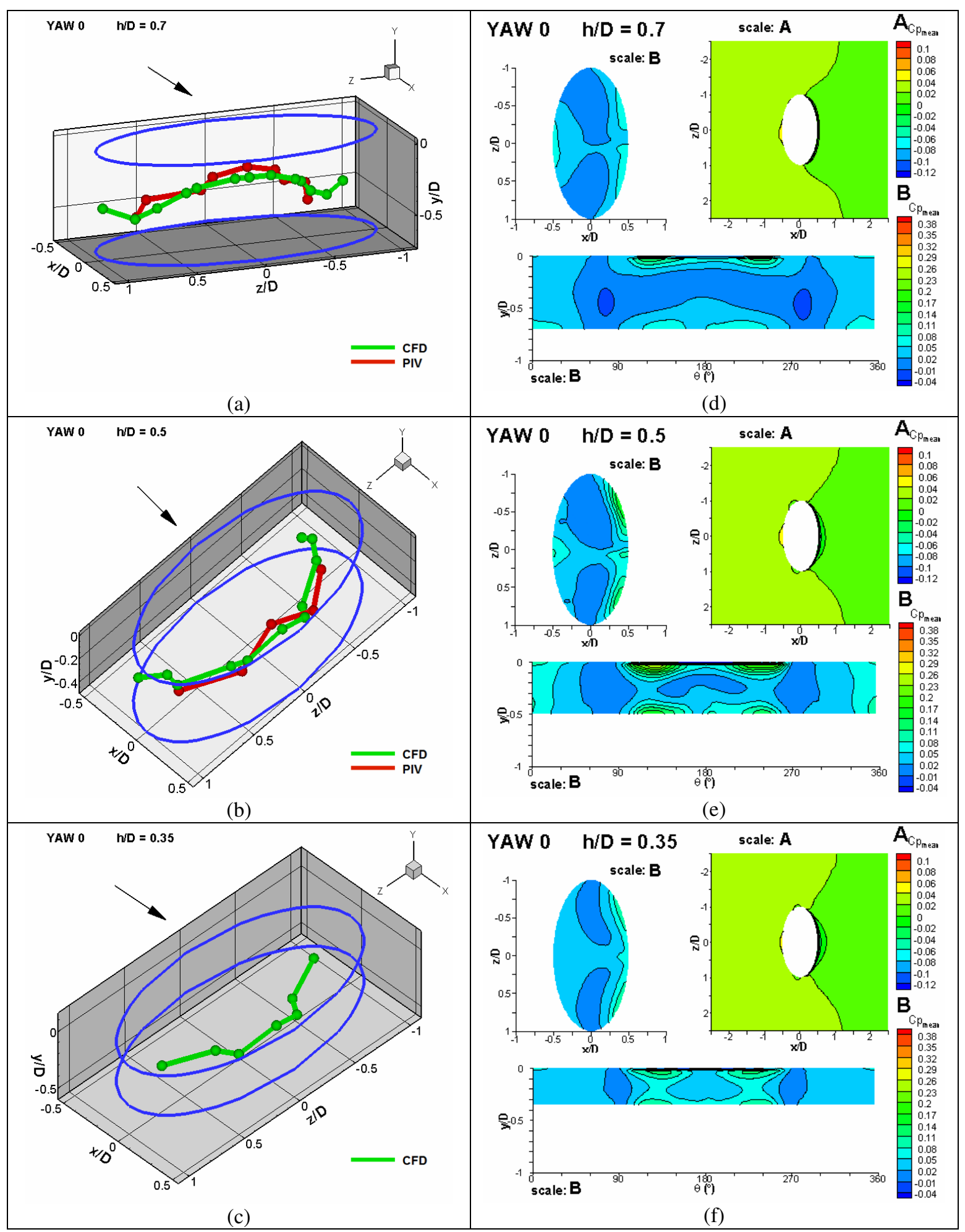

Figure 5-12 Vortex core positions and $C_{p}$ contours at yaw angle $=0^{\circ}$ for $h / D=0.7,0.5$, and 0.35 


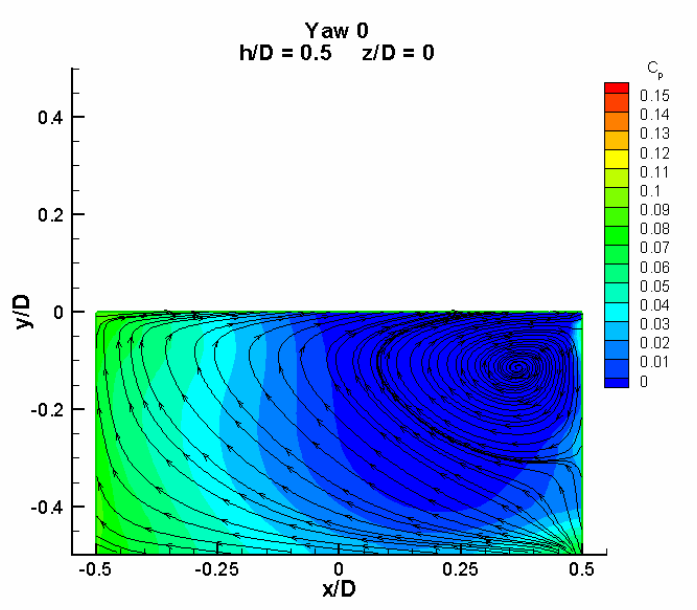

(a)

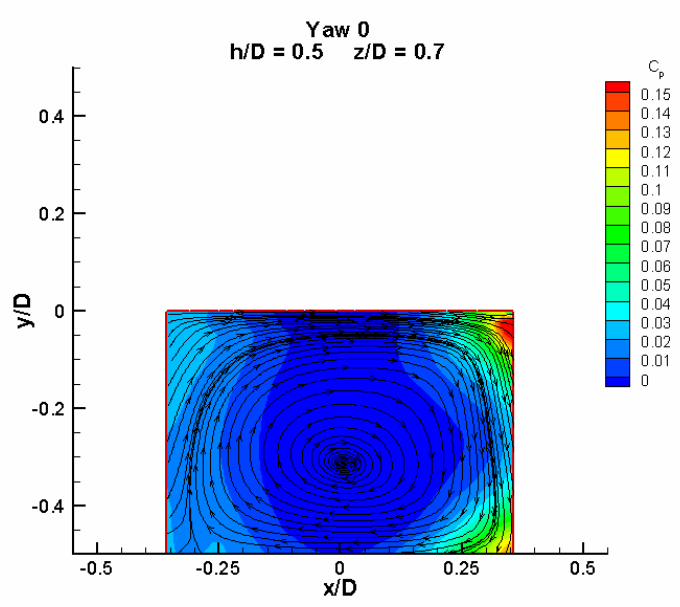

(b)

Figure 5-13 Sectional streamlines and $C_{p}$ contours for cavity with $\mathrm{h} / \mathrm{D}=0.5$ at $0^{\circ}$ yaw angle at $\mathrm{z} / \mathrm{D}=0$ (a) and $\mathrm{z} / \mathrm{D}=0.7$ (b)

The two low pressure regions at the angular positions of $60^{\circ}$ and $300^{\circ}$ indicate the locations where the extreme ends of the vortex core are bound by the upstream wall. Pressure distribution on the cavity floor also shows two separate low-pressure regions, which are due to the outer part of the vortex cores in each cell approaching the cavity floor. Unlike the case of the deep cavity $(\mathrm{h} / \mathrm{D}=1.0)$, the centre part of the vortex core is closer to the downstream wall of the cavity. The vortex core positions and the lowpressure regions on the downstream cavity wall also show that the centre part of the vortex core forms at an increasingly higher position, when the cavity depth decreases $(y / D=-0.2$ for $h / D=0.35$ compared to $y / D=-0.4$ for $h / D=0.7)$. This behaviour indicates the increased upward deflection of the downstream part of the shear layer, observed in Figure 5-9 for these three cases, is related to the location of the centre part of the vortex core.

In the work of Maull and East [16], a two-cell vortex core has been reported for a rectangular cavity with $\mathrm{h} / \mathrm{D}=0.5$, and $\mathrm{W} / \mathrm{D}=2$, exposed to a turbulent boundary layer.

Czech [49] has also confirmed the existence of the cellular structures, in his study of three-dimensional rectangular cavities exposed to a turbulent boundary layer. Figure 5-14 shows the pressure distribution in a rectangular cavity with $\mathrm{W} / \mathrm{D}=3.18$ and an $\mathrm{h} / \mathrm{D}=0.5$, 
at $0^{\circ}$ yaw angle. The pressure distribution indicates that the vortex inside the cavity has a two-cell structure. However, for the cavity with an aspect ratio of 2:1, the results reported by Czech do not show any cellular structure in the cavity vortex. Similarly, in the study by Savory et al. [48], no particular indication of a cellular structure in the cavity vortex was found, for a rectangular cavity with an aspect ratio of 2:1 exposed to a turbulent upstream boundary layer.

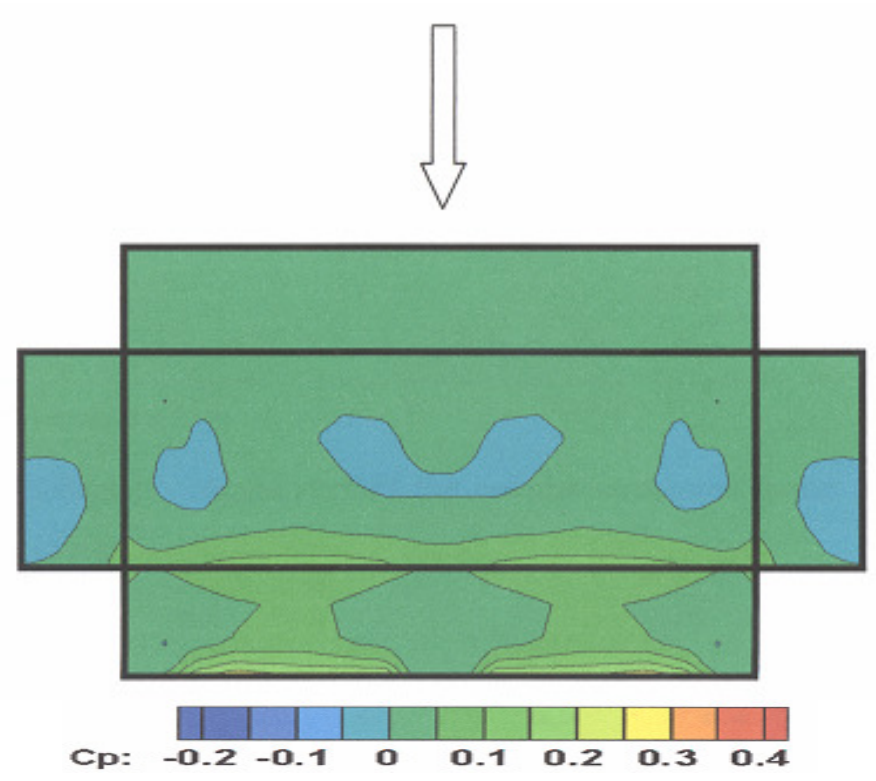

Figure 5-14 $C_{p}$ contours for a rectangular cavity $(W / D=3.18$

$\& h / D=0.5$ ) at $0^{\circ}$ yaw angle (adapted from ref. [49])

For the cavity with a 2:1 elliptical planform, existence of the cellular structure can also be observed in the results of the experiments by Hering [58]. Figure 5-15-(a), which shows pressure distributions in the cavity with $\mathrm{h} / \mathrm{D}=0.5$ at $0^{\circ}$ yaw angle, also confirm the cellular structure of the cavity vortex, with two distinct cells visible [58]. The same behaviour, for a similar configuration, was also observed by Savory and Toy [5]. As shown in Figure 5-15-(b), two distinct high pressure areas exist on downstream wall of the cavity. However, the pressure is slightly lower compared to Hering's experiments and the present numerical simulations. This may be a result of the difference in the thickness of the approaching boundary layer, since they performed their experiments with a thicker boundary layer, $\delta / \mathrm{D}=4.57$ as opposed to $\delta / \mathrm{D}=1.08$ used by Hering and for the present 
simulations. As explained in section 2.4, the thicker boundary layer would result in a lower $C_{p}$ value as lower velocity fluid would be entrained into the cavity from the shear layer spanning the cavity.

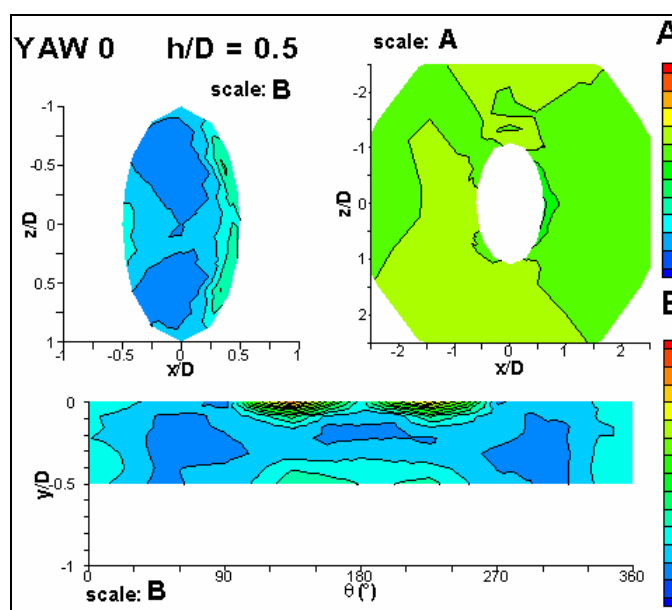

(a)
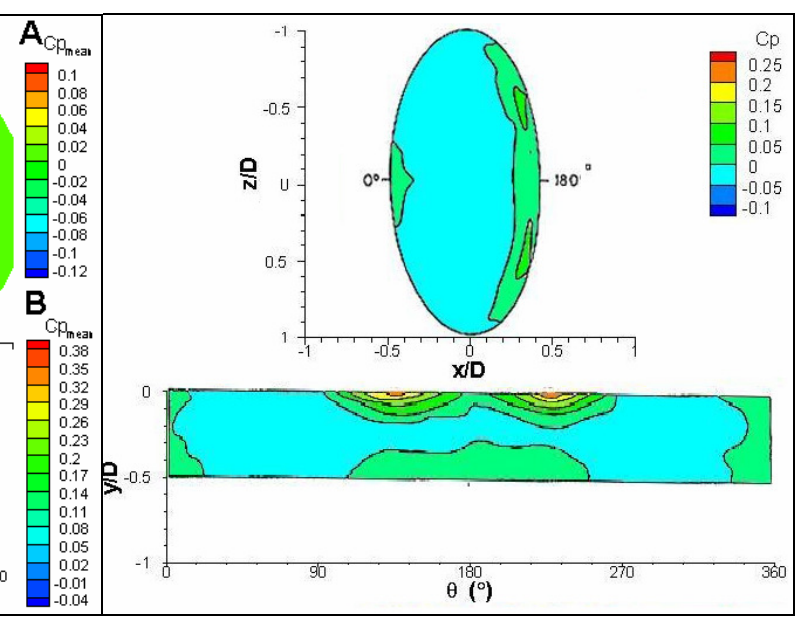

(b)

Figure 5-15 $C_{p}$ contours at yaw angle $=0^{\circ}$ for $h / D=0.5$, Hering

[58] (a), Savory and Toy (adapted from ref. [5]) (b)

The results presented in the previous paragraphs indicate that when the geometric proportions of the cavity are close to those reported by Maull and East [16], the cavity vortex shows a cellular structure for both rectangular and elliptical planform shapes. The existence of a similar $\mathrm{W}$-shaped vortex in the present cavity suggests that the cellular nature of the vortex may be the result of an inherent feature of the recirculation zone in cavities with aspect ratios greater or equal to 2 and intermediate depths, independent of the cavity planform shape.

A noteworthy trend observed in the case of deep and intermediate-depth cavities is variation of the position of the vortex core with cavity depth. A comparison of Figure 5-10 and Figure 5-13 indicates that the vortex core in the mid-plane is farther from the downstream wall for deeper cavities $(\mathrm{x} / \mathrm{D}=-0.2$ for $\mathrm{h} / \mathrm{D}=1.0$ compared to $\mathrm{x} / \mathrm{D}=0.4$ for $\mathrm{h} / \mathrm{D}=0.5$ ). A similar trend can be observed in the case of a nominally two-dimensional rectangular cavities exposed to a turbulent boundary layers, in the studies by Ukeiley and Murray [26], which involves cavities with $\mathrm{h} / \mathrm{D}=0.2$ and 0.7 , and the study by Kang et al. 
[122], which involves cavities with $\mathrm{h} / \mathrm{D}=0.5$ and 1.0. The results of both studies indicate that the vortex core moves towards the upstream wall of the cavity when the cavity depth increases.

\subsubsection{Shallow and transitional cavities $(h / D=0.1$ and 0.2$)$}

When the cavity depth is further decreased to $h / D=0.2$, another significant change in the vortex core positions and surface pressure distributions is found to occur. An examination of the vortex structure at this depth (Figure 5-16-(a)) indicates that a vortex core is formed near the upstream edge of the cavity, and parallel to it. The vortex core is indicative of shear layer separation from the upstream lip of the cavity wall, which causes a recirculation zone to form near the upstream wall of the cavity. The separation vortex is bounded by the curved cavity walls at angular positions of $135^{\circ}$ and $225^{\circ}$, approximately, as indicated by pressure coefficient distribution on the cavity sidewalls (Figure 5-16-(c)). The flow then leaves the cavity, forming another recirculation zone with a vortex that is bounded by the downstream wall. This recirculation zone is related to the upward deflection of the shear layer centre near the downstream wall of the cavity, observed in Figure 5-9. While the general flow structure for $\mathrm{h} / \mathrm{D}=0.2$, is comparable to that of rectangular cavities in the "closed" flow regime, shown in Figures 2-2-(a) and 2-5-(a), due to the existence of the two recirculation zones, one particular difference with the "closed" flow regime is that no high-pressure region indicative of the impingement of the shear layer is found on the cavity base. Instead, a high-pressure region is observed near the edge of the downstream wall, which indicates that the shear layer is entrained inside the cavity, but does not impinge the cavity base. This flow structure is a characteristic of the "transitional-closed" flow regime [14], and represents a transition between the cellular structure and shallow cavity regimes. The streamtraces in the cavity mid-plane are shown in comparison with those of a nominally two-dimensional cavity exposed to a turbulent upstream boundary layer in the "transitional-closed" regime, and show the similarities between the two flows (Figure 5-17). In both cases two recirculation zones exist near the upstream and downstream walls of the cavity, and the shear layer does not impinge upon the cavity base [119]. 


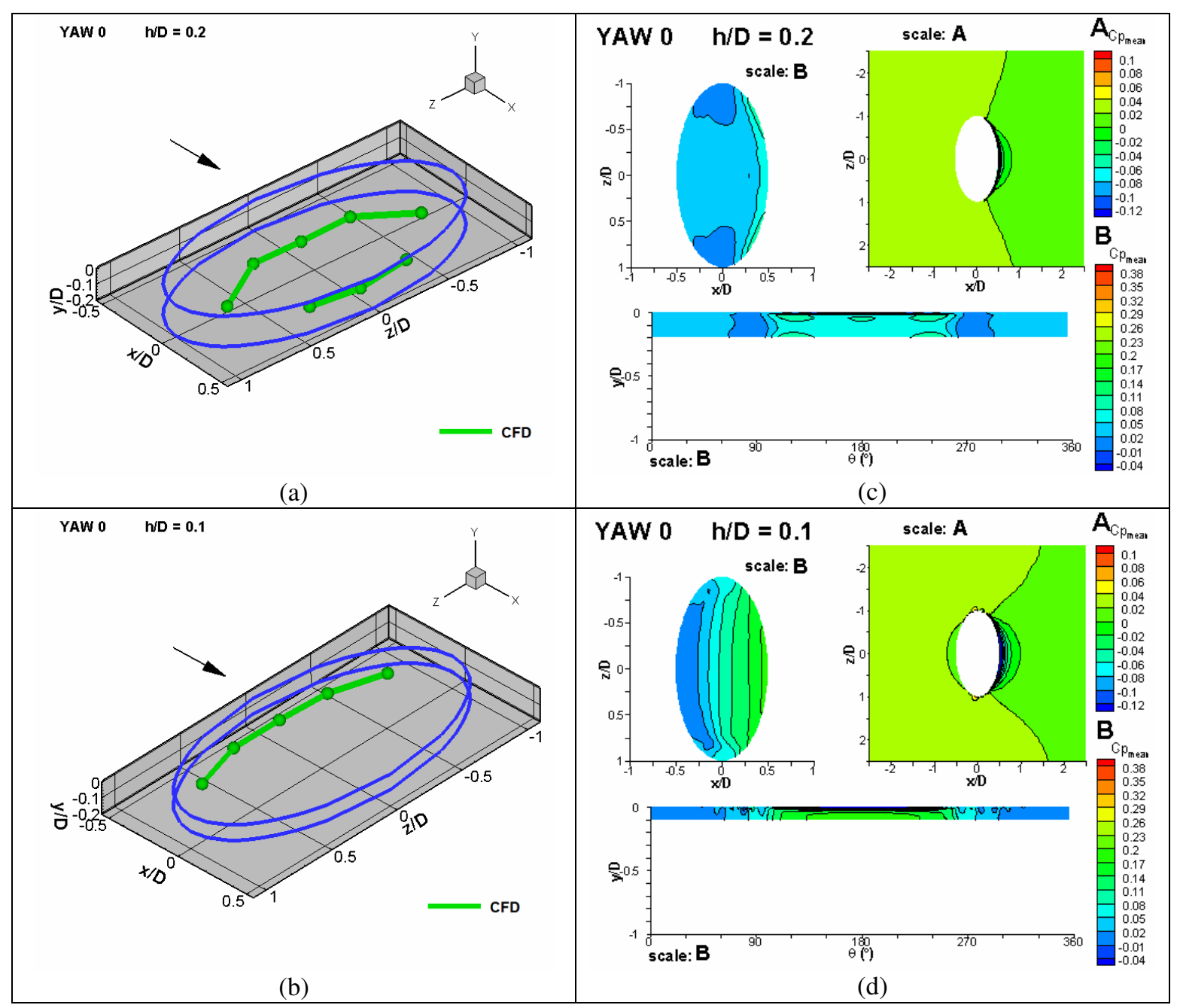

Figure 5-16 Vortex core positions and $C_{p}$ contours at yaw angle $=0^{\circ}$ for $h / D=0.2$ and 0.1

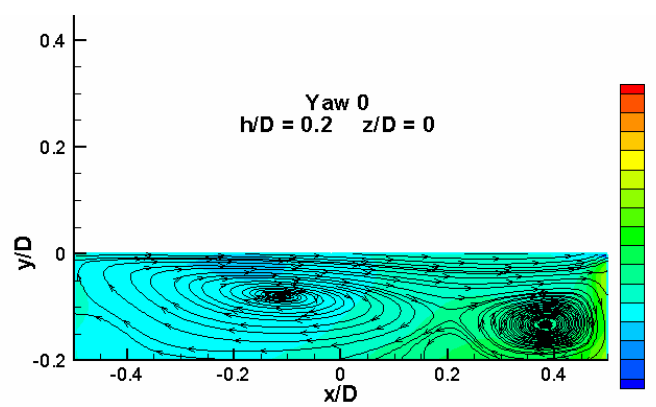

(a)

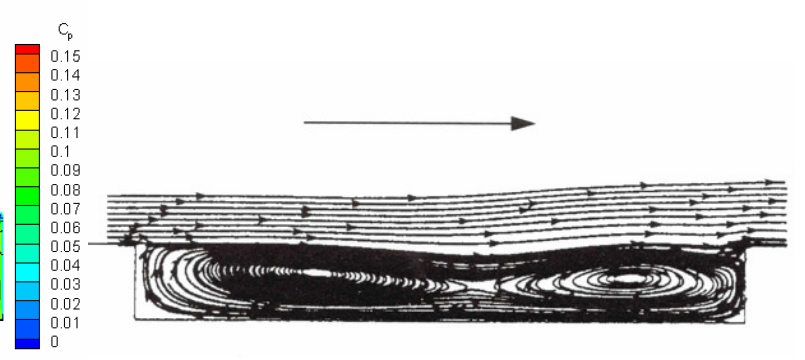

(b)

Figure 5-17 Streamlines and $C_{p}$ contours of cavity with $h / D=0.2$ at $0^{\circ}$ yaw angle (a) and instantaneous streamlines of a nominally two-dimensional rectangular cavity with $h / D=0.13$ [119] 
Finally, for the case of $h / D=0.1$, the vortex core position is indicative of shear layer separation from the upstream lip of the cavity wall, which causes a recirculation zone to form near the upstream wall of the cavity. The formation of this recirculation zone is confirmed by the pressure distributions in Figure 5-16-(d). The location of the vortex core in the primary recirculation zone is approximately $0.25 \mathrm{D}$. No trace of a secondary recirculation zone is found further downstream, according to the vortex core positions and pressure distributions, indicating that the second recirculation zone is very small (Figure 5-18). However, the pressure increases on the cavity base, towards the downstream wall. This is followed by a low pressure region on the boundary layer plate, immediately downstream of the downstream edge of the cavity wall. This pressure distribution indicates that although the shear layer does not impinge the cavity base, it enters the cavity volume and approaches the base, and then leaves the cavity volume, forming a separation region at the edge of the downstream cavity wall. This behaviour is consistent with the position of the shear layer centre for this case (Figure 5-9), and can be associated with the "transitional-closed" regime [14], The location of the vortex core in the primary recirculation zone is approximately $0.25 \mathrm{D}$ from the upstream cavity wall, which agrees with the location of the low pressure region in the surface pressure distributions.

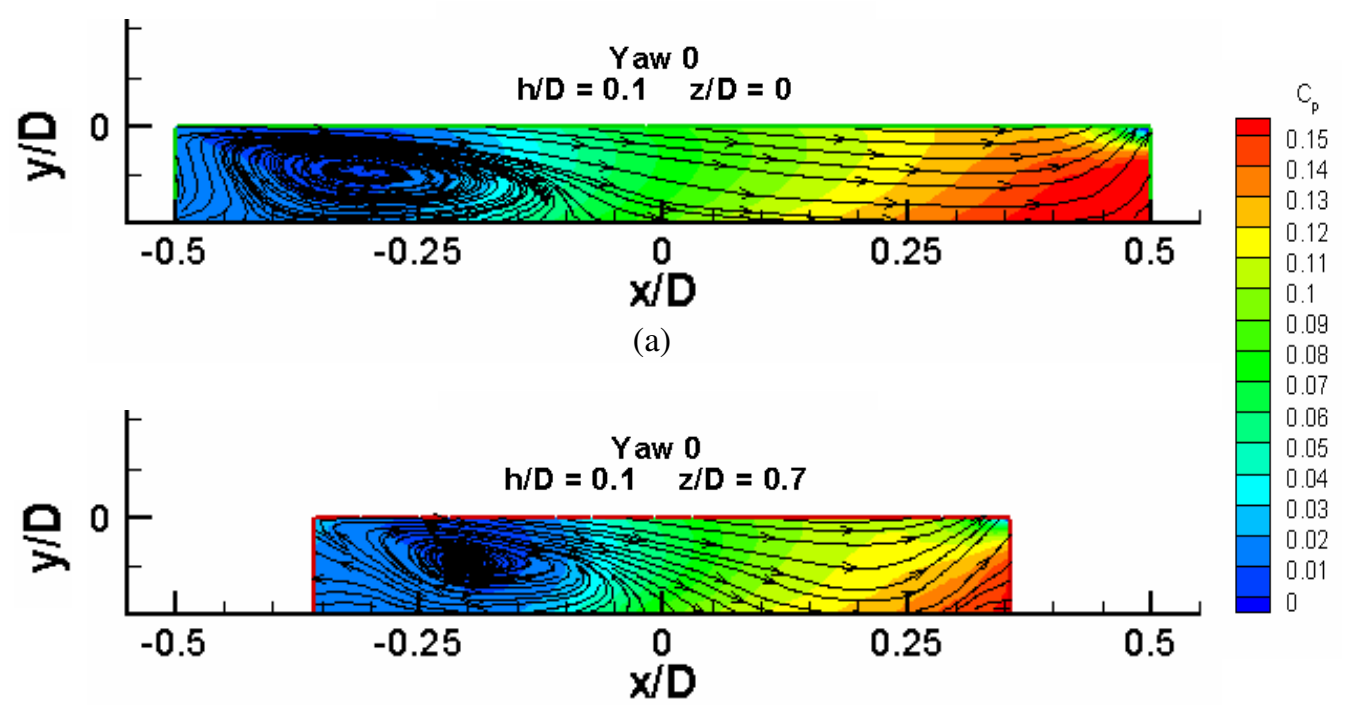

(b)

Figure 5-18 Streamlines and and $C_{p}$ contours for cavity with $h / D=0.1$ at $0^{\circ}$ yaw angle at $\mathrm{z} / \mathrm{D}=0$ (a) and $\mathrm{z} / \mathrm{D}=0.7$ (b) 
The pressure coefficient contours based on the experiments by Hering [58] are shown in Figure 5-19 for $\mathrm{h} / \mathrm{D}=0.1$. A comparison between Figure 5-19 and Figure 5-16-(d) indicates similar patterns in pressure distributions, suggesting a similar flow structure.

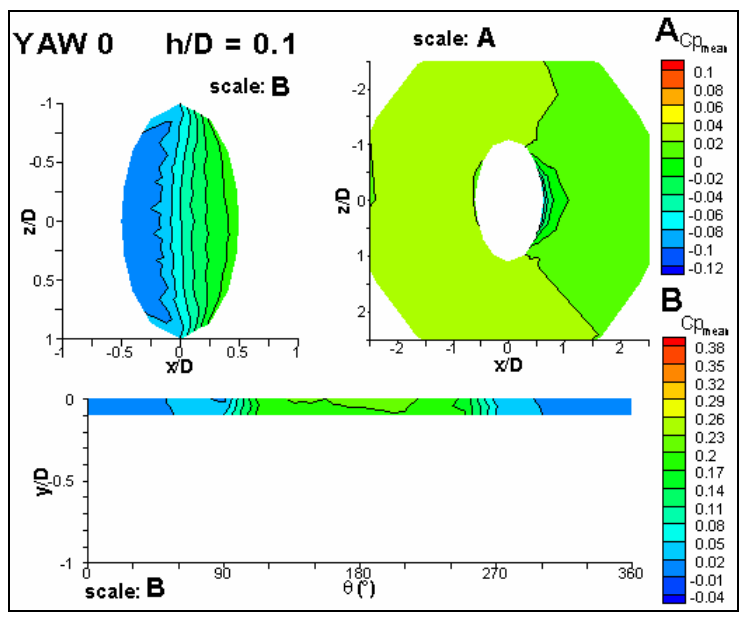

Figure 5-19 $C_{p}$ contours at yaw angle $=0^{\circ}$ for $h / D=0.1[58]$

\subsubsection{Drag Characteristics}

The relationship between the drag of the cavity and its flow structure is investigated in the following paragraphs. Normalized drag coefficients $\left(\frac{\Delta C_{D}}{c_{f}}\right)$, obtained based on the present numerical stimulations, are plotted in Figure 5-20, for cavities with h/D ratios between 0.1 and 1.0 at $0^{\circ}$ yaw angle. The figure also includes data from other studies involving rectangular and elliptical cavities with aspect ratio of 2:1, including the studies by Friesing [3] and Savory et al. [5] and [48], and circular cavities, including the studies by Savory et al. [52] and Dybenko and Savory [53]. The results of the present study are close to the values reported by Friesing [3] and within the uncertainty limit of the values reported by Hering [58], except for $\mathrm{h} / \mathrm{D}=0.5$. Compared to other results for the same configuration [3] and [18], Hering's results for $\mathrm{h} / \mathrm{D}=0.5$ and 0.7 show smaller values of drag coefficient.

A general trend can be observed in Figure 5-20, in which drag coefficient are lower for rectangular and elliptical cavities with larger h/D. In the case of rectangular cavity, 
Savory et al. [48] have attributed the larger drag in the case of shallow cavities to the increased entrainment of the shear layer compared to deep cavities, which leads to formation of a larger high pressure region associated with shear layer impingement upon the downstream wall of the cavity, closer to the cavity base. This explanation is consistent with the results of the present study, in which lower positions of the shear layer centre, and larger high-pressure regions are observed for shallow and transitional cavities with smaller $\mathrm{h} / \mathrm{D}$ ratios. The drag coefficients for circular cavities do not follow this trend, and indicate a very high value for the cavity with $\mathrm{h} / \mathrm{D}=0.5$, which has been attributed to strong asymmetry in the flow [53].

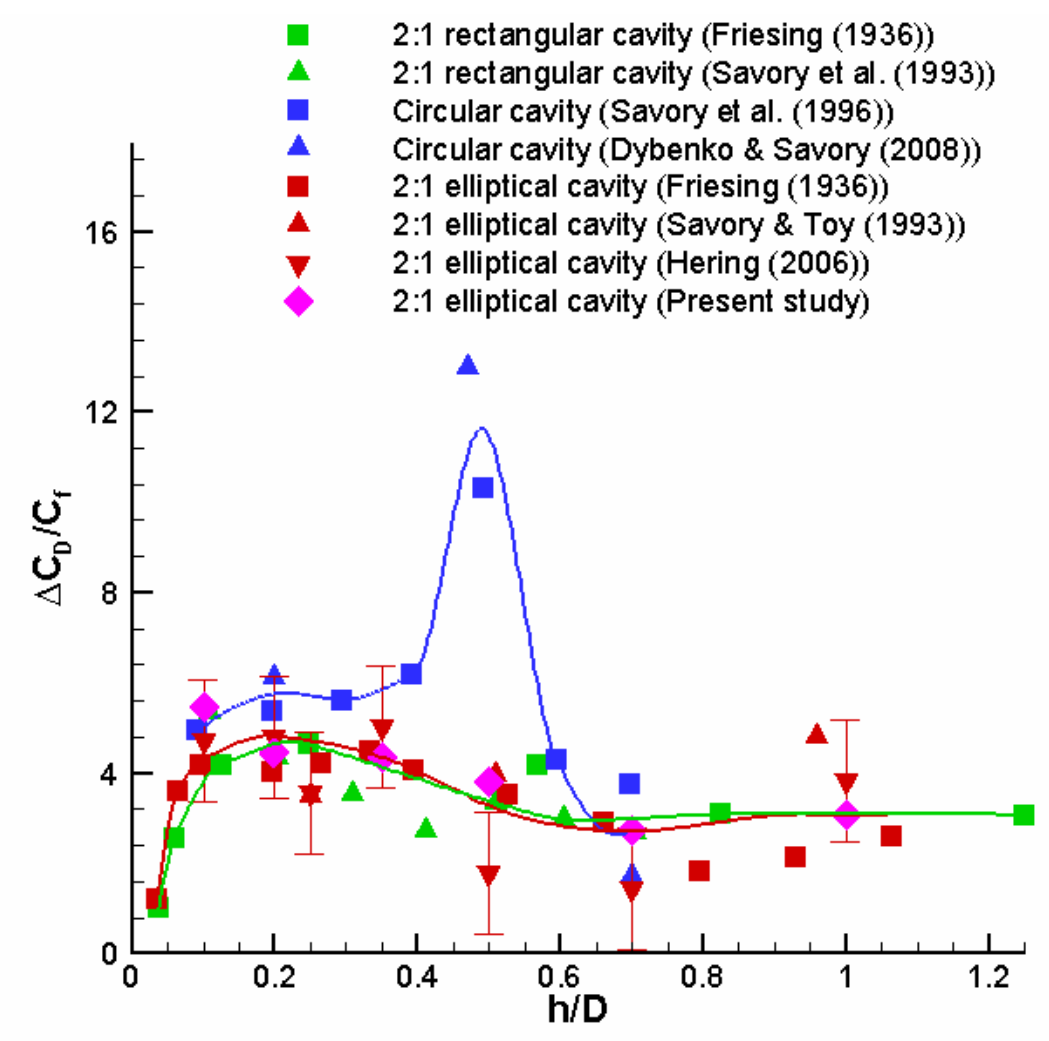

Figure 5-20 Comparison of drag coefficient at yaw angle $=0^{\circ}$ (The lines are added to visualize the general trend for each planform shape.)

\subsubsection{Flow Downstream of the Cavity}

Separation of the flow from the upper edge of the downstream wall of the cavity is one of the features of the flow structure of elliptical cavities in symmetric flow regimes. As 
discussed in Section 5-3-2, the shear layer separated from the upstream edge of the cavity impinges upon the downstream wall of the cavity and forms a region of high pressure near the upper edge of the wall. The flow then exits the cavity volume, by turning around the edge of the cavity. Due to the sharp, 90-degree corner of the edge, the flow separates at this point. For all h/D ratios, evidence of the flow separation can be observed in the pressure distributions shown in section 5.3.2, as a low-pressure region, formed on the boundary layer plate immediately after the downstream of edge of the cavity.

Effect of this separation on the flow downstream of the cavity is studied in this section for $\mathrm{h} / \mathrm{D}$ ratios of $0.7,0.5$, and 0.2 , based on velocity distributions in horizontal and vertical planes at various locations downstream of the cavity. These cases have been selected to make it possible to use the results reported by Hering [58] for the same h/D ratios for comparison.

Figure 5-21 shows distribution of velocity deficit in vertical planes located at the positions of $x / D=0.5$ and $x / D=2.6$, based on the results of the numerical simulations of the present study, and the experiments by Hering [58]. Velocity deficit is determined by subtracting the velocity in absence of the cavity at the similar downstream position, from the measured or computed velocities, using the following equation:

$$
\frac{\Delta \bar{u}}{U_{\mathrm{inf}}}=\left(\frac{\bar{u}}{U_{\mathrm{inf}}}\right)_{\text {Cavity }}-\left(\frac{\bar{u}}{U_{\mathrm{inf}}}\right)_{\text {No Cavity }}
$$

The figures show one dominant low velocity region at $\mathrm{z} / \mathrm{D}=0$ for cavities with $\mathrm{h} / \mathrm{D}=0.7$ and $h / D=0.5$. For the cavity with $h / D=0.2$, the results of the numerical simulations show two low velocity regions located approximately at $\mathrm{z} / \mathrm{D}= \pm 0.2$. These velocity distributions can be explained by a close examination of the separated flow region downstream of the cavity, and its position relative to the vertical plane located at $\mathrm{x} / \mathrm{D}=$ 0.5 . 


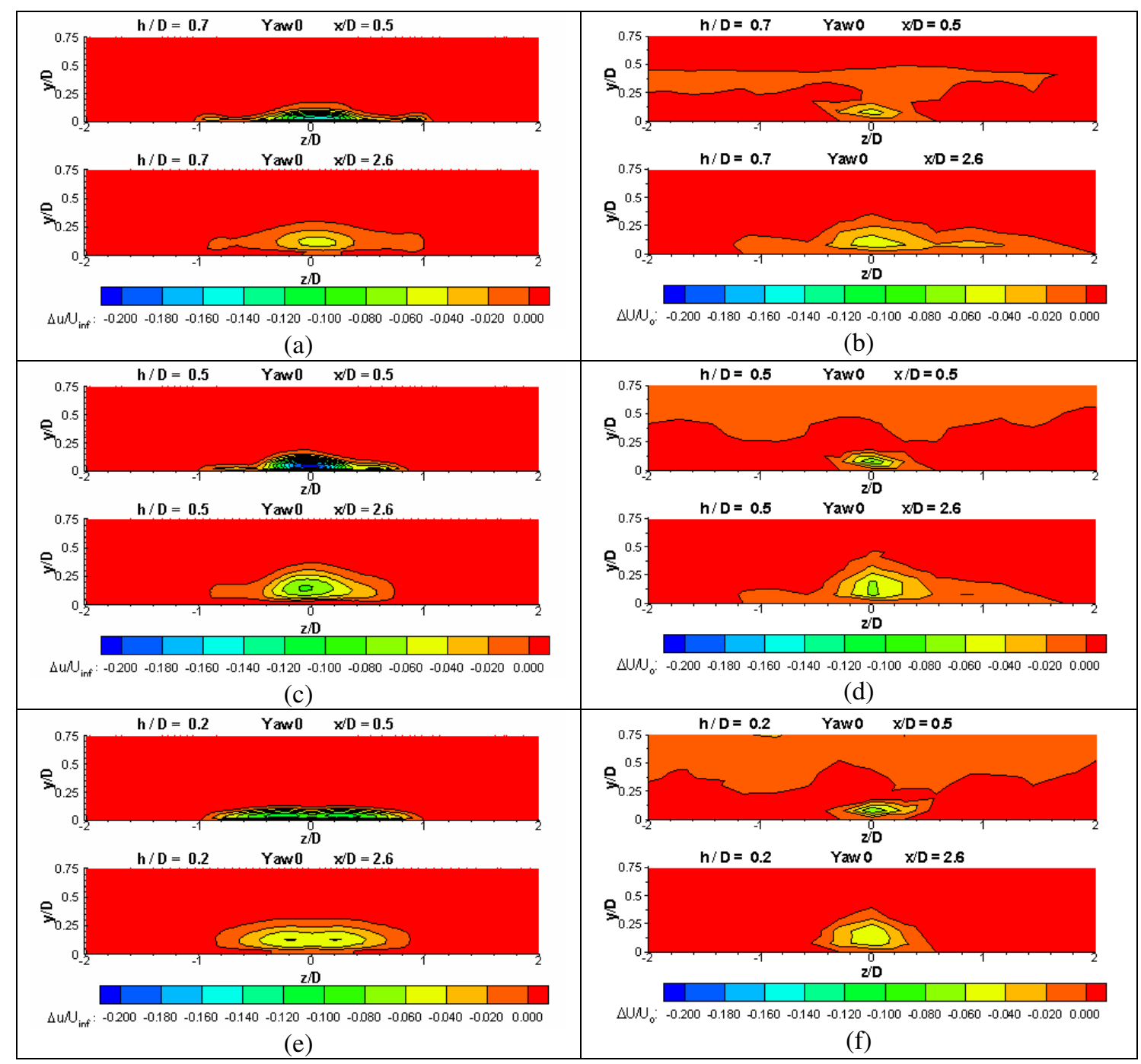

Figure 5-21 Velocity deficit contours based on numerical simulations (left) and experimental measurements [58] (right) at $0^{\circ}$ yaw angle

As it can be observed in Figure 5-12 and Figure 5-16, a low-pressure region associated with the separated flow is visible downstream of the edge of the cavity for all three cases. For the cavity with $\mathrm{h} / \mathrm{D}=0.2$, the low-pressure region covers a larger extent in the downstream direction, compared to the cases of $\mathrm{h} / \mathrm{D}=0.5$ and 0.7 . This can be explained by comparing the position of the shear layer for the three cases. As discussed in section 5.3.1 and shown in Figure 5-9, the shear layer centre is at a lower position for the cavity with $\mathrm{h} / \mathrm{D}=0.2$, compared to the other two cases. Therefore, a larger part of the shear layer is entrained inside the cavity with $\mathrm{h} / \mathrm{D}=0.2$. Considering that the shear layer contains velocities larger than the flow inside the cavity, the downstream wall of the 
cavity will be exposed to a larger oncoming velocity at the impingement region compared to deeper cavities with less shear layer entrainment. The flow in this region is similar to that of a forward facing step exposed to an oncoming turbulent shear layer. For this type of flow, Sherry et al. [120] have shown that when the maximum velocity of the shear layer impinging onto the step increases by a factor of 3 , the length of the recirculation zone formed downstream of the edge of the step increases by $50 \%$. This observation is consistent with the present finding, in which a larger recirculation zone is formed downstream of the cavity with $\mathrm{h} / \mathrm{D}=0.2$.

In the case of the cavity with $\mathrm{h} / \mathrm{D}=0.2$, the vertical plane located at $\mathrm{x} / \mathrm{D}=0.5$ intersects with the larger and more curved separation region, at two locations at spanwise positions of $\mathrm{z} / \mathrm{D}= \pm 0.2$. Two low velocity regions can be seen in the vertical plane at the same spanwise locations. However, in the case of the cavities with $\mathrm{h} / \mathrm{D}=0.5$ and 0.7 , the vertical plane is almost tangent to the narrower separation region at $\mathrm{z} / \mathrm{D}=0$, and one low velocity region is observed.

For the cavities with $\mathrm{h} / \mathrm{D}=0.7$ and 0.5 , two smaller low velocity regions can also be observed at $\mathrm{z} / \mathrm{D}= \pm 1.0$, approximately. These low velocity regions might be associated with side edge vortices, which are formed due to the effect of the shear layer being entrained into the cavity following separation from the edge of the upstream wall, as described in section 2-3. Figure 5-16 shows two small low-pressure regions at the two corners of the cavity $(\mathrm{z} / \mathrm{D}= \pm 1.0)$, which further support the possibility of formation of side edge vortices. Due to their small size at $\mathrm{x} / \mathrm{D}=0.5$, the low velocity regions have not been detected in the measurements by Hering [58]. However, at $x / D=2.6$, where they are found to have expanded, traces of them are visible in both the results of Hering and the present numerical results. No trace of the small low-velocity regions can be observed for the cavity with $\mathrm{h} / \mathrm{D}=0.2$ in Figure 5-21, indicating that the side edge vortices are weak or non-existent. This is in accordance with previously reported findings about side edge vortices [12], in which the side-edge vortices are found to be weaker for cavities with smaller depth to width ratios, due to less entrainment of the flow over the side edges of the cavity. 
Figure 5-22, shows the velocity distribution based on PIV measurements, in a horizontal plane located $3 \mathrm{~mm}$ above the upper edge of the cavity $(\mathrm{y} / \mathrm{D}=0.04)$, extending between $\mathrm{x} / \mathrm{D}=0.5$ and $\mathrm{x} / \mathrm{D}=2.7$ in the streamwise direction, and between $\mathrm{z} / \mathrm{D}=-1.0$ and $\mathrm{z} / \mathrm{D}=$ 1.0 in the spanwise direction. Both parts (a) and (b) of the figure show a low velocity region extending downstream of the cavity. It should be mentioned that the circular arc is due to masking of imaging area by the joint between the base plate enclosing the cavity, and the ground plate (see Figure 4-2). Considering the uniform spanwise velocity distribution upstream of the cavity (section 4.4), the reason for the slightly asymmetric velocity deficit about the centreline for both cases is presumably a small error in the setting of the yaw angle to $90^{\circ}$ in the experiment, or a slight non-uniformity in the cavity model.

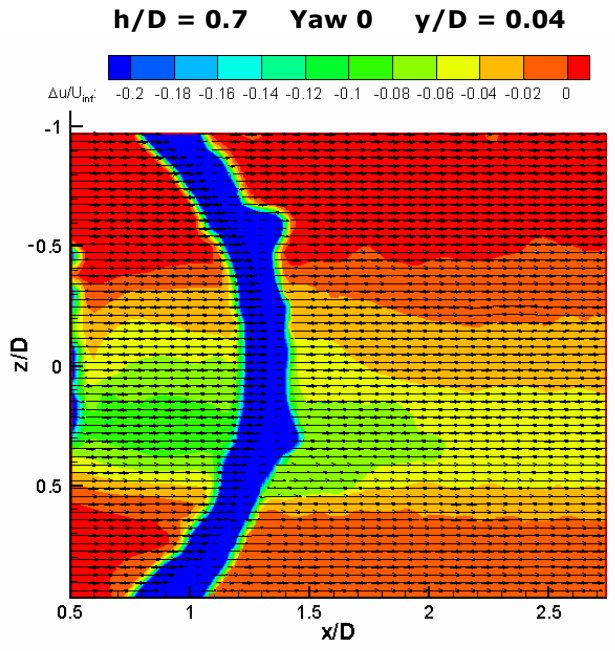

(a)

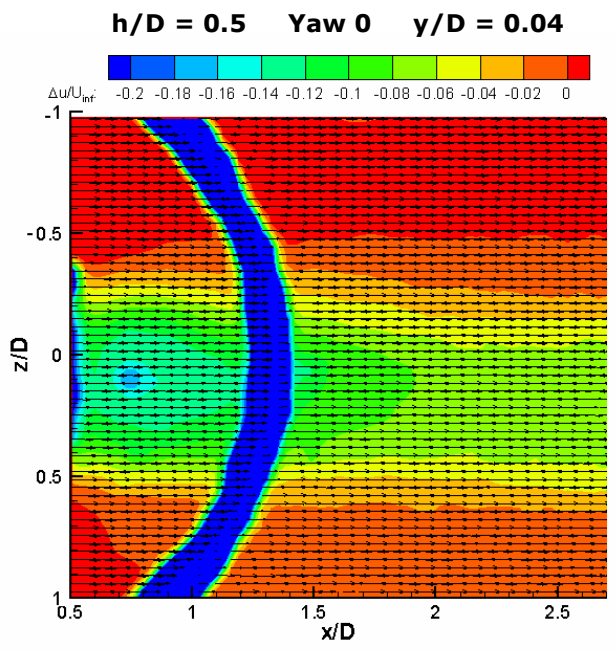

(b)

Figure 5-22 Velocity deficit contours based on PIV measurements at $0^{\circ}$ yaw angle in a horizontal plane

Both Figure 5-21 and Figure 5-22 show that the low velocity region expands in the spanwise and vertical directions as the distance from the downstream edge of the cavity increases.

A comparison of the velocity deficits in the vertical planes for the three depths (Figure 5-21) shows that the largest velocity deficit is caused by the cavity with $\mathrm{h} / \mathrm{D}=0.5$. 
Comparison of parts (a) and (b) of Figure 5-22 also shows that the cavity with $\mathrm{h} / \mathrm{D}=0.5$ has a larger velocity deficit.

Figure 5-23 compares the spanwise variations of $\frac{\Delta \bar{u}}{U_{\text {inf }}}$ at $\mathrm{x} / \mathrm{D}=0.65$ for cavities with $\mathrm{h} / \mathrm{D}$ $=0.7$ and 0.5 based on numerical simulations and PIV measurements. $\mathrm{x} / \mathrm{D}=0.65$ was chosen because it was not possible to extract reliable data at $\mathrm{x} / \mathrm{D}=0.5$ due to reflections from the cavity wall. The results of numerical simulations and PIV measurement show similar peak velocity deficits and the same trend. However, the spanwise position of this peak is offset from $\mathrm{z} / \mathrm{D}=0$ in the case of experimental results due to the slight deviation of the yaw angle from $0^{\circ}$. This figure also confirms that the cavity with $\mathrm{h} / \mathrm{D}=0.5$ has a velocity deficit larger than that of $\mathrm{h} / \mathrm{D}=0.7$.

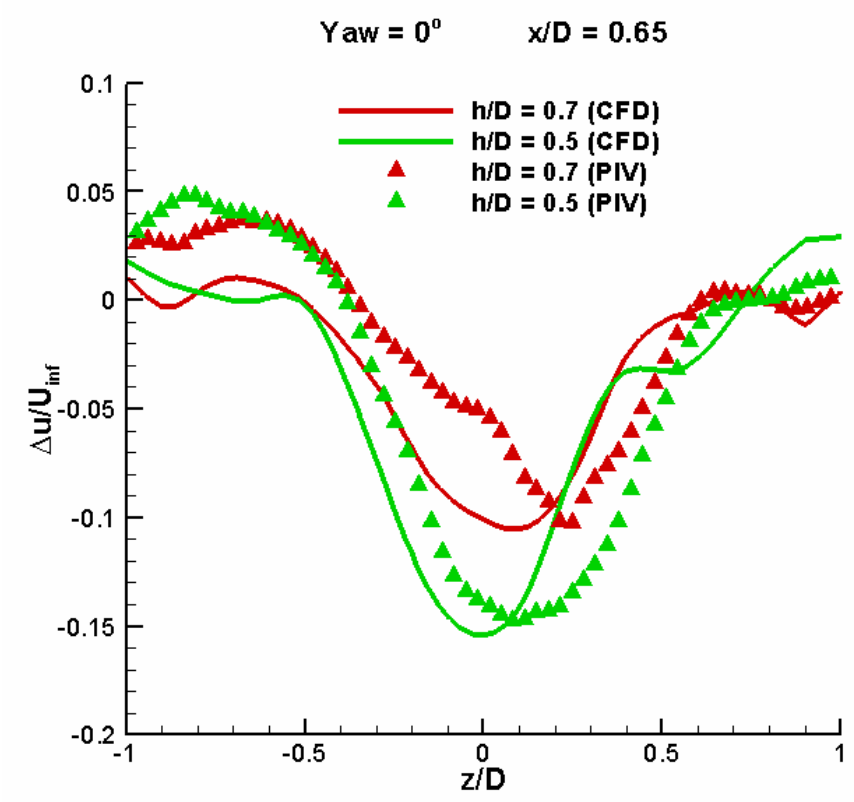

Figure 5-23 Quantitative comparison of velocity deficit at $0^{\circ}$ yaw angle

In the case of the cavity with $\mathrm{h} / \mathrm{D}=0.5$, the shear layer centre has the highest position near the downstream edge of the cavity wall, among all h/D ratios investigated (Figure 5-9). On the other hand the thickness of the shear layer near the downstream edge of the cavity is almost similar for all cases (Figure 5-8). Therefore, a larger part of the shear 
layer is outside the cavity for the cavity with $h / D=0.5$. Since the velocity in the shear layer is smaller than the freestream velocity, a larger velocity deficit will exist downstream of the cavity in this case. The larger velocity deficit in the wake of the cavity with $h / D=0.5$ is consistent with the larger drag for this case, compared to the cavity with $\mathrm{h} / \mathrm{D}=0.7$ (Figure 5-20).

Existence of vortex structures in the flow downstream of the cavities at $0^{\circ}$ yaw angle was also investigated, using the numerical results. No trace of vortex structures was observed, except for a very small region of streamwise vorticity $\left(\omega_{x}\right)$ immediately downstream of the edge of the cavity, which was found to diminish in the streamwise direction (Figure 5-24). More explanation about these regions of streamwise vorticity will be given in section 5.4.4. It should be noted that throughout this chapter, positive values of $\omega_{x}$ indicate streamwise vorticity in the downstream (positive-x) direction, with circulation in clockwise direction looking downstream.
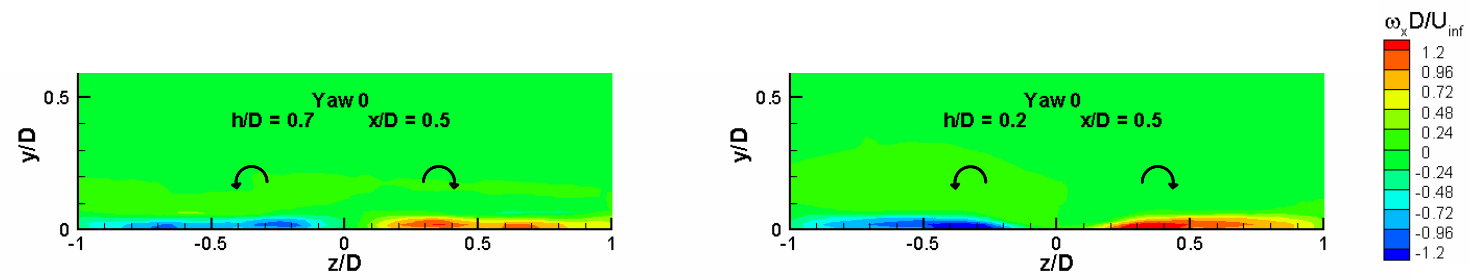

Figure 5-24 Streamwise vorticity contours at $x / D=0.5$ for $\mathrm{h} / \mathrm{D}=0.7$ (a) and $\mathrm{h} / \mathrm{D}=\mathbf{0 . 5}(\mathrm{b})$ at $\mathrm{0}^{\circ}$ yaw angle

\subsubsection{Flow Dynamics}

Previous studies in which flow in cavities with various depths are investigated have shown that a relationship exists between cavity depth and the level of flow oscillations inside the cavity. The results of these studies suggest that the vortex inside the cavity is more stable for deeper cavities. In the case of a nominally two-dimensional rectangular cavity exposed to a turbulent upstream boundary layer with $\operatorname{Re}_{D}=2.7 \times 10^{5}$, Ukeiley and Murray [26] have shown that when the cavity depth increases from $h / D=0.2$ to $h / D=$ 0.7 , the level of fluctuations in streamwise velocity (indicated by $\mathrm{u}_{\mathrm{rms}}$ ) in the vicinity of the vortex core decreases from $0.2 \mathrm{U}_{\text {inf }}$ to $0.1 \mathrm{U}_{\text {inf. }}$. A similar reduction is also observed in 
$\mathrm{V}_{\text {rms }}$, which decreases from $0.12 \mathrm{U}_{\text {inf }}$ to $0.07 \mathrm{U}_{\text {inf. Pressure measurements by Hering [58] }}$ also indicate that the maximum level of pressure fluctuations on the cavity walls for the cavity with $\mathrm{h} / \mathrm{D}=1.0$ is smaller than those of cavities with smaller $\mathrm{h} / \mathrm{D}$ ratios. As an example, for the cavities in the symmetric flow regime (yaw $=0^{\circ}$ ), the maximum level of $\mathrm{C}_{\text {Prms }}$, which is associated with the location of shear layer impingement upon the downstream wall of the cavity, decreases from 0.04 to 0.01 , when cavity depth is increased from $h / D=0.5$ to $h / D=1.0$. While the results reported by Hering [58] are in accordance with the trend observed by Ukeiley and Murray [26], they are limited to surface pressure distributions, and do not provide an insight into the behaviour of the vortex in the cavity.

In the following paragraph, this behaviour will be investigated in more detail in the case of elliptical cavities in the symmetric flow regime, by comparing the flow in cavities with $\mathrm{h} / \mathrm{D}=0.5$ and $\mathrm{h} / \mathrm{D}=1.0$.

As mentioned earlier, the level of velocity fluctuations, indicated by $u_{\mathrm{rms}}$ and $v_{\mathrm{rms}}$, is an indicator of flow oscillations in the cavity. Distribution of $\mathrm{u}_{\mathrm{rms}}$ in the cavity mid-plane $(\mathrm{z} / \mathrm{D}=0)$ are shown in Figure 5-25, for cavities with $\mathrm{h} / \mathrm{D}=0.5,0.7$, and 1.0. The figure indicates increasingly higher levels of $\mathrm{u}_{\mathrm{rms}}$ in the cavity for smaller depth. A quantitative comparison of the distributions of $\mathrm{u}_{\mathrm{rms}}$ and $\mathrm{v}_{\mathrm{rms}}$ on a vertical line located at $\mathrm{x} / \mathrm{D}=0, \mathrm{z} / \mathrm{D}=$ 0 , which are shown in Figure 5-26 confirms this trend, and indicates larger x-velocity fluctuations for the cavity with $\mathrm{h} / \mathrm{D}=0.5$, compared to those with $\mathrm{h} / \mathrm{D}=0.7$ and 1.0. In the case of $y$-velocity fluctuations, the maximum value is almost the same for both $h / D=$ 0.7 and 0.5 and larger than that of $\mathrm{h} / \mathrm{D}=1.0$. It should be mentioned that, for better comparability, cavity depth (h) is used to normalize the vertical axis.

In order to gain a better insight into the dynamic behaviour of the flow inside the cavity, and to investigate how the velocity fluctuations are related to this dynamic behaviour, Principal Orthogonal Decomposition (POD) analysis has been used to analyze the dynamics of the results of the PIV velocity field measurements. Details of the formulation of POD analysis are described in section 4.12. 


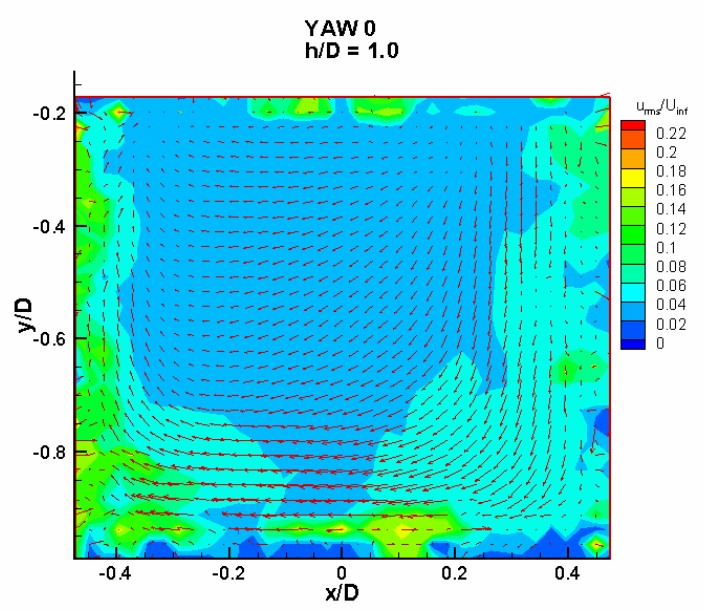

(a)

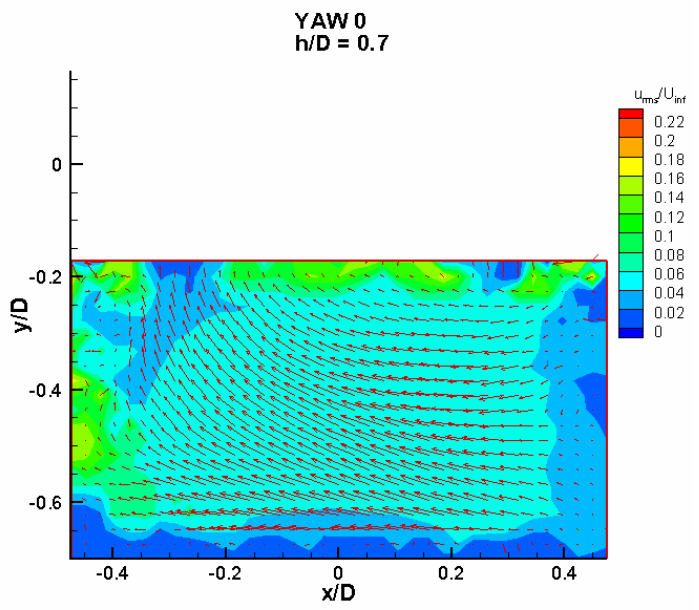

(b)

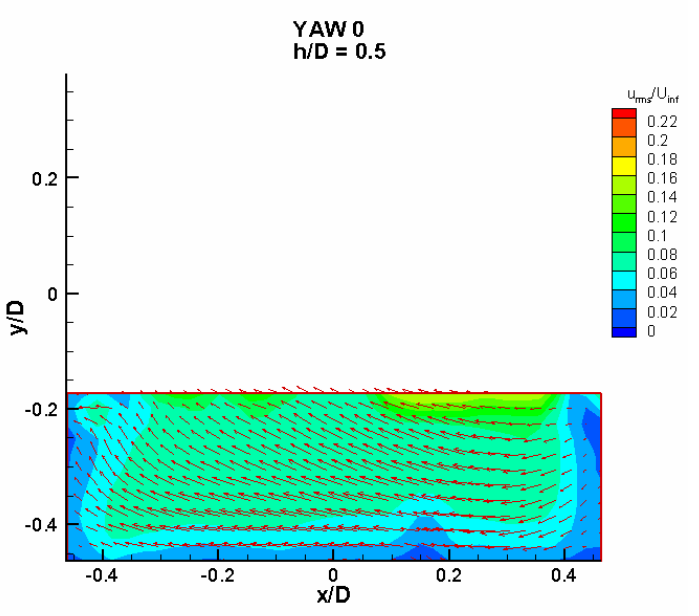

(c)

Figure 5-25 $u_{\text {rms }}$ contours for $h / D=1.0(a), 0.7(b)$, and $0.5(c)$ at $0^{\circ}$ yaw angle at $\mathrm{z} / \mathrm{D}=0$

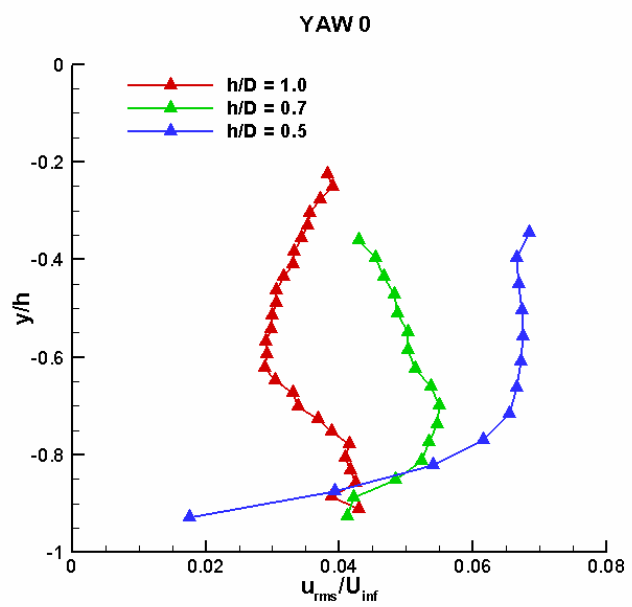

(a)

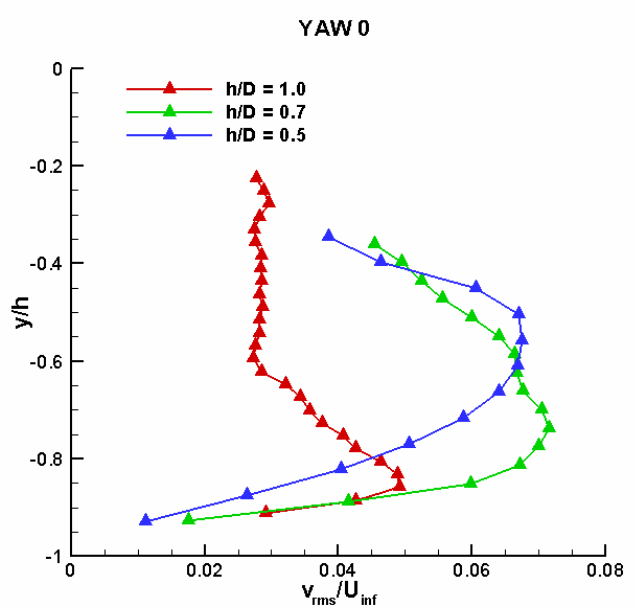

(b)

Figure 5-26 Comparison of $u_{\mathrm{rms}}(\mathrm{a})$ and $v_{\mathrm{rms}}$ (b) profiles on the vertical centreline 
POD analysis has been carried out on the PIV data for cavities with $\mathrm{h} / \mathrm{D}=0.5$ and $\mathrm{h} / \mathrm{D}=$ 1.0 , in the vertical plane located at $\mathrm{z} / \mathrm{D}=0.35$. This vertical plane has been chosen based on the flow structures described in section 5.3.2, to make sure that the measurement area contains the vortex core.

Figure 5-27 and Figure 5-28 show the first four POD modes of the fluctuating streamwise and vertical velocity components, respectively, for the cavity with $h / D=0.5$. $\mathrm{A}$ comparison of the mode shapes indicates that a relationship exists between the spatial distributions of the velocity fluctuations in the shapes of the first two modes, in which the two modes are distinguished by a spatial shift in the regions of high and low fluctuating velocities. This behaviour suggests that Modes 1 and 2 point to the same dynamic behaviour in the flow and, therefore, a temporal relationship is expected to exist between them.
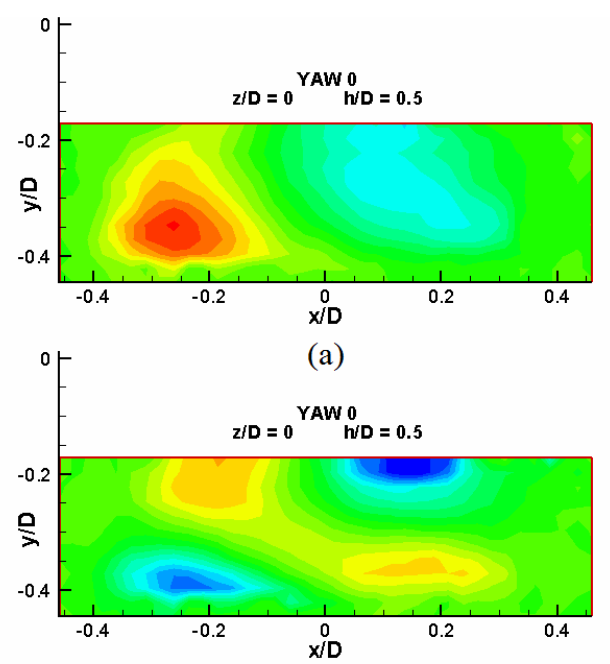

(c)

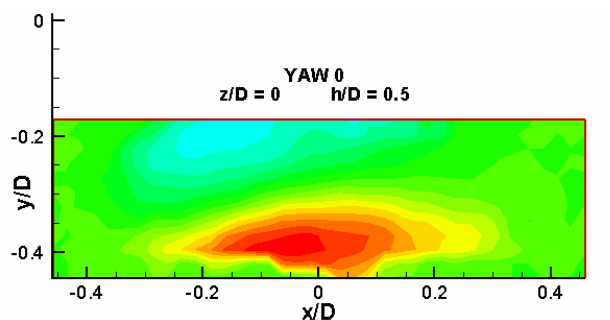

(b)

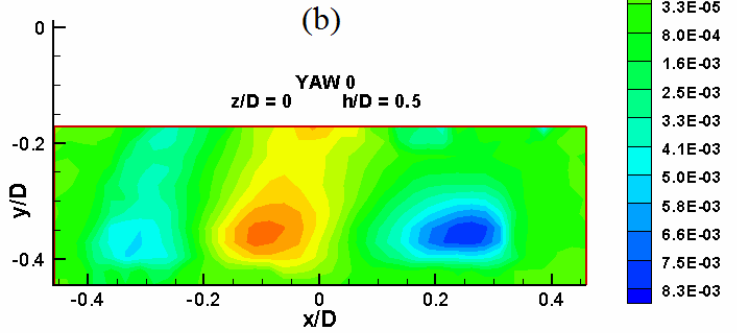

(d)

Figure 5-27 The first four POD modes of the fluctuating streamwise velocity component

The relative energies of the POD modes, which are represented by the normalized eigenvalues $\left(\lambda_{i} / \sum_{i=1}^{n} \lambda_{i}\right)$, are shown in Figure 5-29 for the cavities with $\mathrm{h} / \mathrm{D}=1.0$ and 0.5 . It can be observed that the first two modes contain the largest part of kinetic energy of the fluctuating velocity field. The energy of the first two modes constitutes $16.5 \%$ and $22.5 \%$ 
of the total energy of the velocity field for $h / D=1.0$ and 0.5 respectively. These ratios are smaller than the ratios of the energy contained by the first two POD modes in the study by Murray et al. [25] involving a nominally two-dimensional rectangular cavity exposed to a turbulent upstream boundary layer, and the study by Savory et al. [35] involving a rectangular cavity, representing an urban street canyon. This can be explained by considering the distribution of velocity fluctuations in the flow field. The shear layer contains most of the dynamic content of the flow field, as the fluctuating velocity components are the highest in that region. This can be clearly observed in the distribution of fluctuating velocity components, an example of which is shown in Figure 5-30. This figure shows the distribution of $v_{\mathrm{rms}}$ from the studies by (a) Oszalp [29] involving a nominally two-dimensional rectangular cavity $(\mathrm{h} / \mathrm{D}=0.5)$, (b) Haigermoser [54], involving a cavity with circular planform $(\mathrm{h} / \mathrm{D}=0.5)$, and (c) the present study, involving the elliptical cavity $(\mathrm{h} / \mathrm{D}=0.7)$ at the vertical mid-plane located at $\mathrm{z} / \mathrm{D}=0$. All the figures indicate that the highest levels of $\mathrm{v}_{\mathrm{rms}}$ occur in the shear layer. However, as shown by Figure 5-30-(c), in the present PIV measurements, most of the shear layer is not included in the measurement area due to the masking caused by the base plate. This explains the smaller relative energies of the first two POD modes in the present results, compared to other studies.
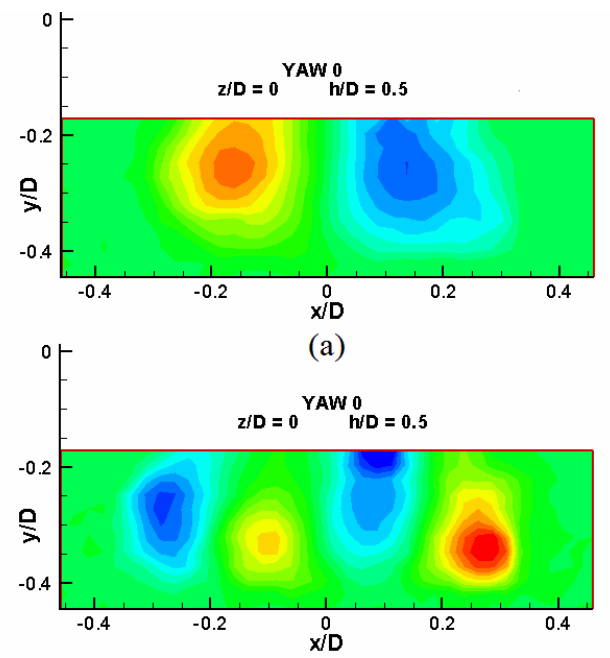

(c)

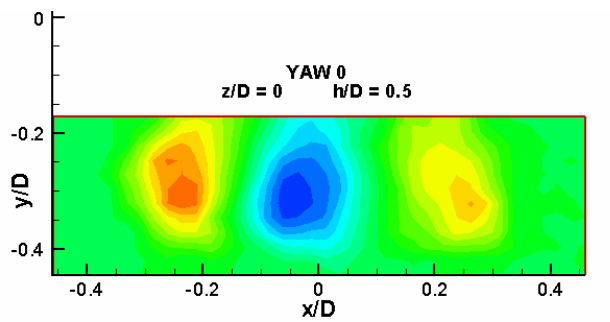

(b)

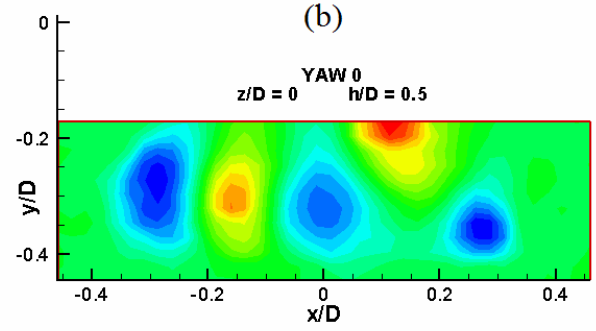

(d)

Figure 5-28 The first four POD modes of the fluctuating vertical velocity component 


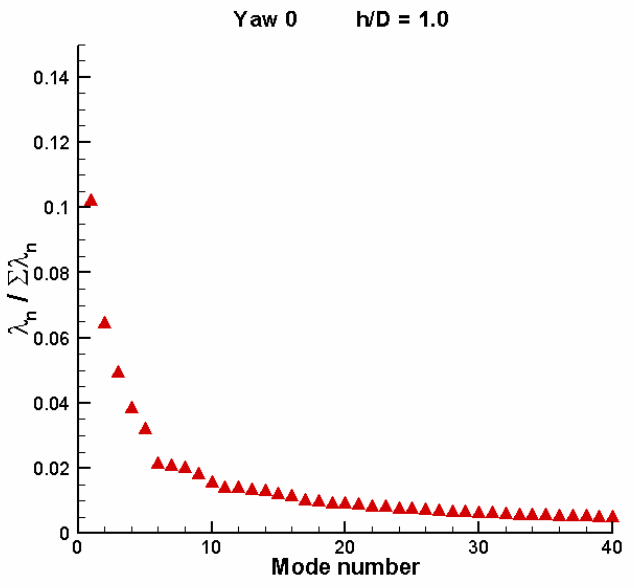

(a)

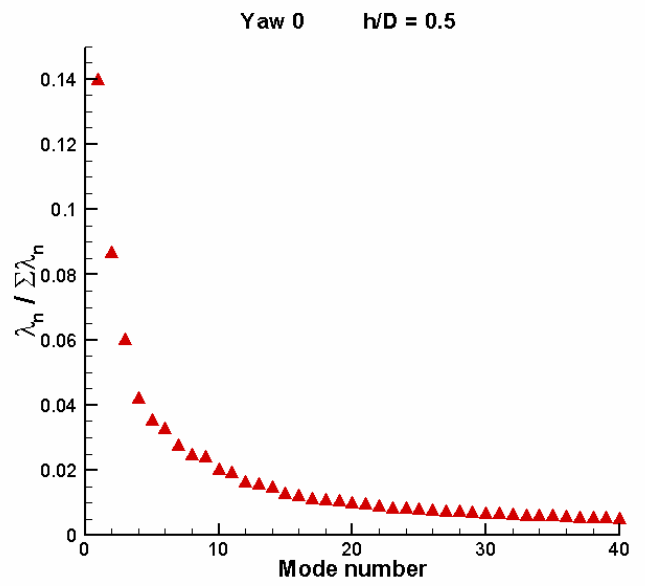

(b)

Figure 5-29 The first forty normalized eigenvalues for $h / D=$

1.0 (a) and 0.5 (b) at $0^{\circ}$ yaw angle at $\mathrm{z} / \mathrm{D}=0$

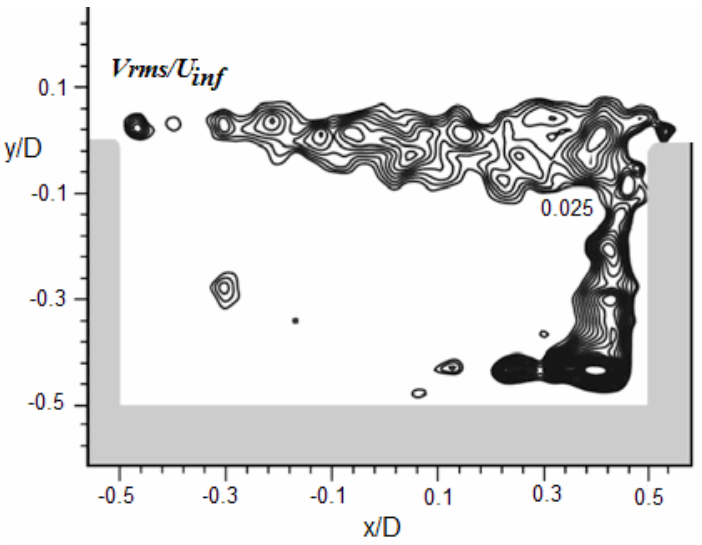

(a)

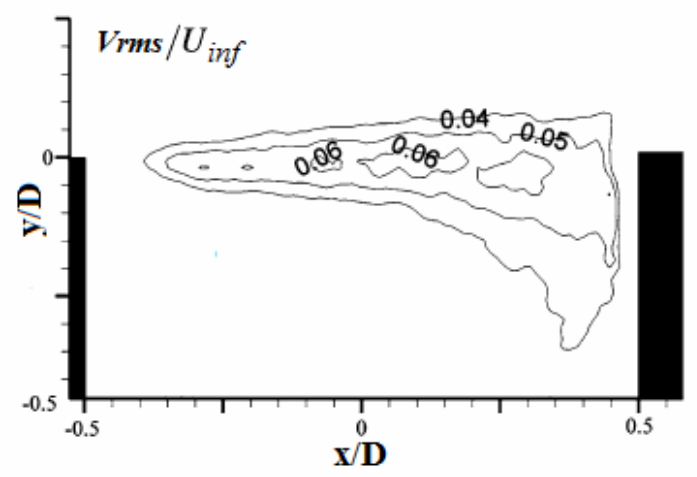

(b)

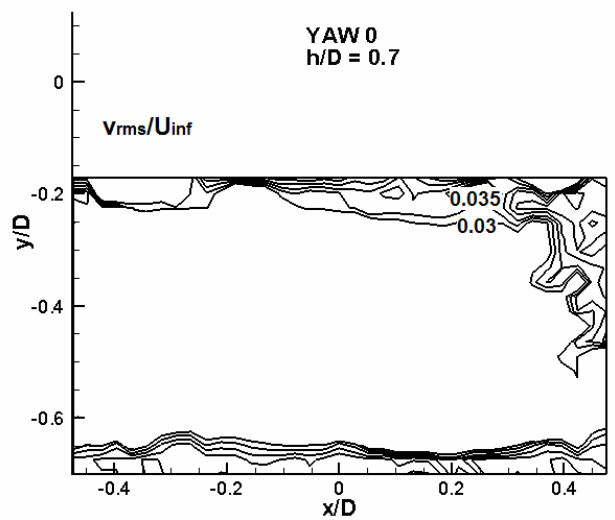

(c)

Figure 5-30 $v_{\mathrm{rms}}$ contours on vertical mid-plane $(\mathrm{z} / \mathrm{D}=0)$ by Oszalp (adapted from ref. [29]) for a two-dimensional rectangular cavity (a), Haigermoser (adapted from ref. [54]) for a circular cavity (b), and the present study (c) 
An investigation of the behaviour of the time varying coefficients of the first two modes, $\mathrm{a}_{1}(\mathrm{t})$ and $\mathrm{a}_{2}(\mathrm{t})$, confirms the temporal relationship between them. Figure 5-31 shows plots of the normalized time-varying coefficients $a_{1}(t) / \sqrt{2 \lambda_{1}}$ vs. $a_{2}(t) / \sqrt{2 \lambda_{2}}$, for the cavities with $\mathrm{h} / \mathrm{D}=1.0$ and 0.5 respectively. Both figures indicate that the normalized time varying coefficients are mostly concentrated in a circular region. This behaviour has been interpreted by previous researchers, including Perrin et al. [121] and Savory et al. [35], as an indication that a 90-degree phase difference exists between the normalized time varying coefficients of Modes 1 and 2.

This relationship can be utilized to determine the phase angle phase $\theta$ for each PIV snapshot using the following equation:

$$
\theta=\tan ^{-1}\left(\frac{a_{2}(t) / \sqrt{2 \lambda_{2}}}{a_{1}(t) / \sqrt{2 \lambda_{1}}}\right)
$$

The phase angles are then used to calculate phase averaged velocity fields, which make it possible to investigate the flow oscillations by comparing the flow structure in various phases, in an approach similar to that implemented by Savory et al. [35] in the case of an urban cavity.

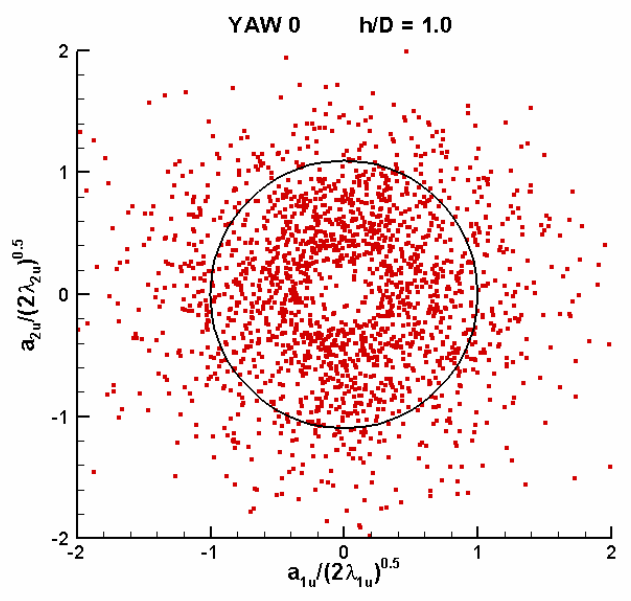

(a)

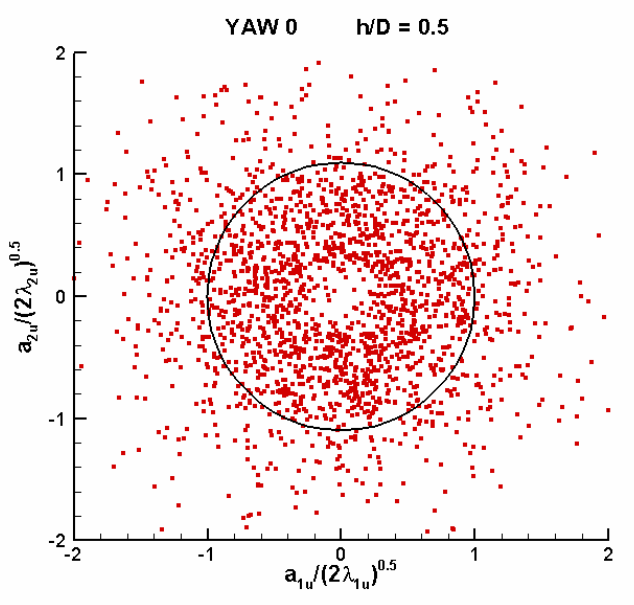

(b)

Figure 5-31 Normalized time-varying coefficients for the cavities with $h / D=1.0$ (a) and 0.5 (b) 
Plots of streamtraces in the cavity based on the phase averaged velocity fields are shown in Figure 5-32 and Figure 5-33, for $\mathrm{h} / \mathrm{D}=1.0$ and 0.5 respectively. The plots show considerable vortex oscillations inside the cavity with $h / D=0.5$. The vortex in the cavity with $\mathrm{h} / \mathrm{D}=1.0$, however, is found to be relatively stable. As a quantitative measure of the oscillations, variations in the position of the vortex core in the streamwise and vertical directions are $0.03 \mathrm{D}$ and $0.04 \mathrm{D}$ for the cavity with $\mathrm{h} / \mathrm{D}=1.0$, and $0.07 \mathrm{D}$ and $0.1 \mathrm{D}$ for the cavity with $\mathrm{h} / \mathrm{D}=0.5$. In previous studies by Haigermoser et al. [24] and Ukeiley and Murray [26], similar oscillations of the vortex have been attributed to flapping of the shear layer, due to an instability which is independent of the resonant conditions, which leads to in- and outflow events (known as breathing). As mentioned before, the present PIV measurements do not allow an investigation of the dynamics of the shear layer itself due to masking of the images, however, the similar vertical oscillations of the vortex, and the fact that the present cavity is not in resonant conditions (section 2.5), suggests that a similar flapping instability in the shear layer is responsible for the vortex oscillations.

The results of the POD analysis indicate that oscillations of the vortex are considerably larger for the intermediate-depth cavity $(\mathrm{h} / \mathrm{D}=0.5)$, compared to the deep cavity $(\mathrm{h} / \mathrm{D}=$ 1.0). This observation agrees with the previously reported results indicating larger surface pressure and velocity fluctuations for cavities with smaller depths, and suggests that the higher fluctuating values are due to increased vortex oscillation. 


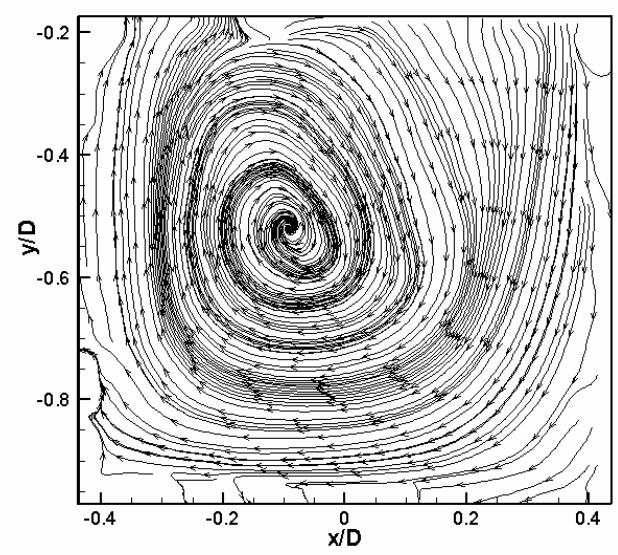

(a)

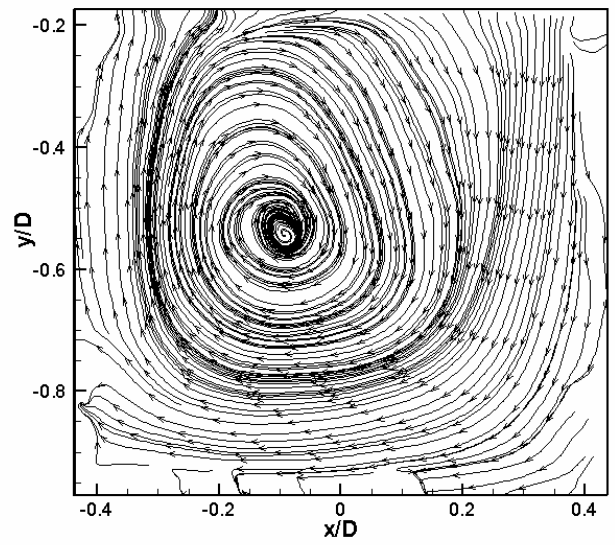

(c)

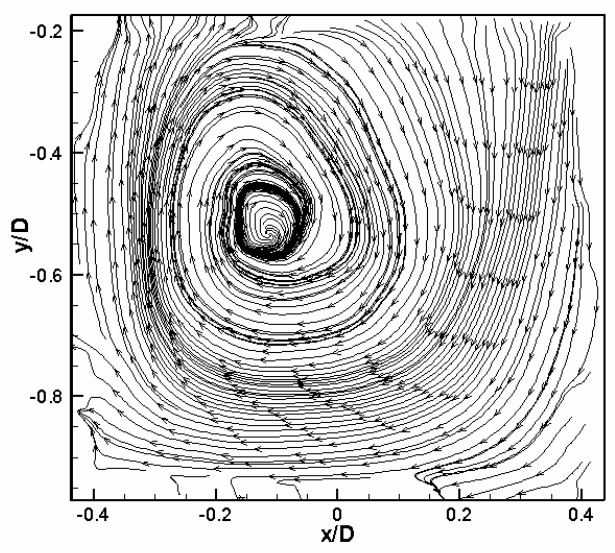

(b)

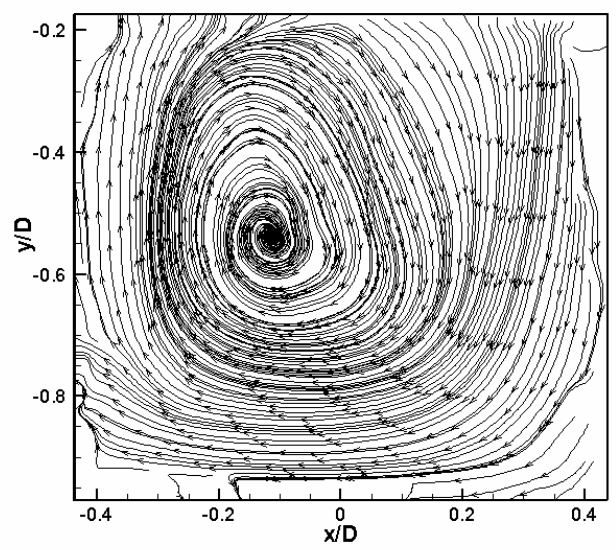

(d)

Figure 5-32 Phase averaged streamtraces in the vertical plane located at $\mathrm{z} / \mathrm{D}=\mathbf{0 . 3 5}$, for the cavity with $h / D=1.0$. The phase interval between consecutive plots is $90^{\circ}$.

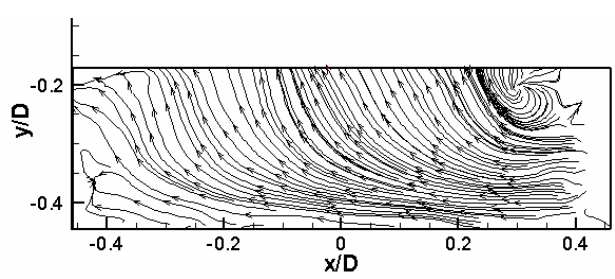

(a)

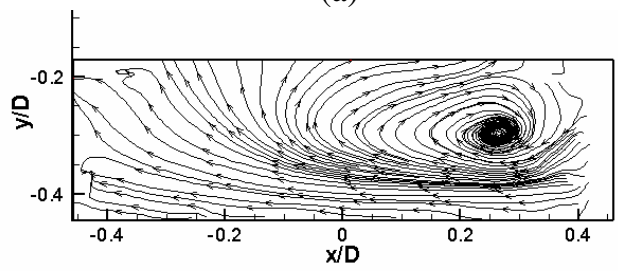

(c)

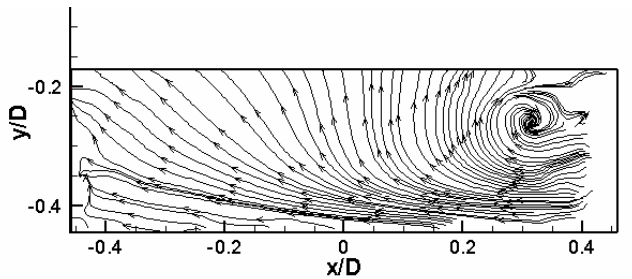

(b)

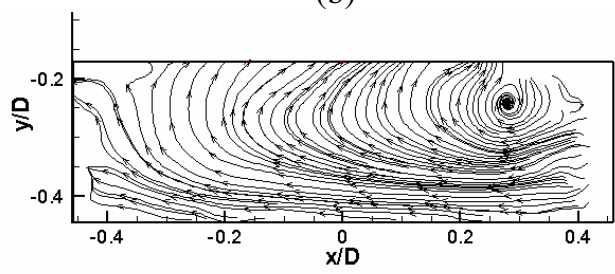

(d)

Figure 5-33 Phase averaged streamtraces in the vertical plane located at $\mathrm{z} / \mathrm{D}=\mathbf{0 . 3 5}$, for the cavity with $h / D=0.5$. The phase interval between consecutive plots is $90^{\circ}$. 


\subsubsection{Summary of Findings for Symmetric Flow Regimes}

The findings presented in this section regarding symmetric flow regimes can be summarized as follows:

- Three distinct flow regimes are observed for cavities at $0^{\circ}$ yaw angle, depending on the h/D ratio. While the mean flow is symmetric in all three regimes, significant changes in flow structures can be observed when the $h / D$ ratio changes.

- For the cavity with $\mathrm{h} / \mathrm{D}=1.0$, behaviour of the shear layer is similar to that of deep nominally two-dimensional rectangular cavities in the "open" flow regime. The shear layer of the cavity with $\mathrm{h} / \mathrm{D}=0.1$ shows a behaviour similar to shallow rectangular cavities in the "closed" flow regime. Between these two depths, behaviour of the shear layer is not similar to either of the two regimes and indicates a different flow structure.

- For all cavity depths in the symmetric regime, the shear layer grows almost linearly downstream of the separation point, with an average growth rate of $d \delta_{\omega} / d x=0.2$, which is within the range of the growth rate observed in other studies on cavity flows.

- The flow structure for the cavity with $\mathrm{h} / \mathrm{D}=1.0$ consists of a single, captive vortex similar to that of deep nominally two-dimensional cavities in the "open" regime. Position and orientation of the vortex core varies across the span, due to three-dimensional effects. Figure 5-34 shows a schematic representation of the main features of the flow structure in this regime

- For cavities with h/D ratios between 0.7 and 0.35 , the flow structure includes a single, captive vortex with a cellular structure. The number of cells for all cases is 2 , which is consistent with the numbers of cells previously observed in the case of cavities with rectangular planforms of the same aspect ratio, indicating that formation of the cellular structures is independent of the cavity planform shape. 
When the cavity depth increases, the vortex core forms at a position closer to the downstream wall, and at a smaller depth (Figure 5-35).

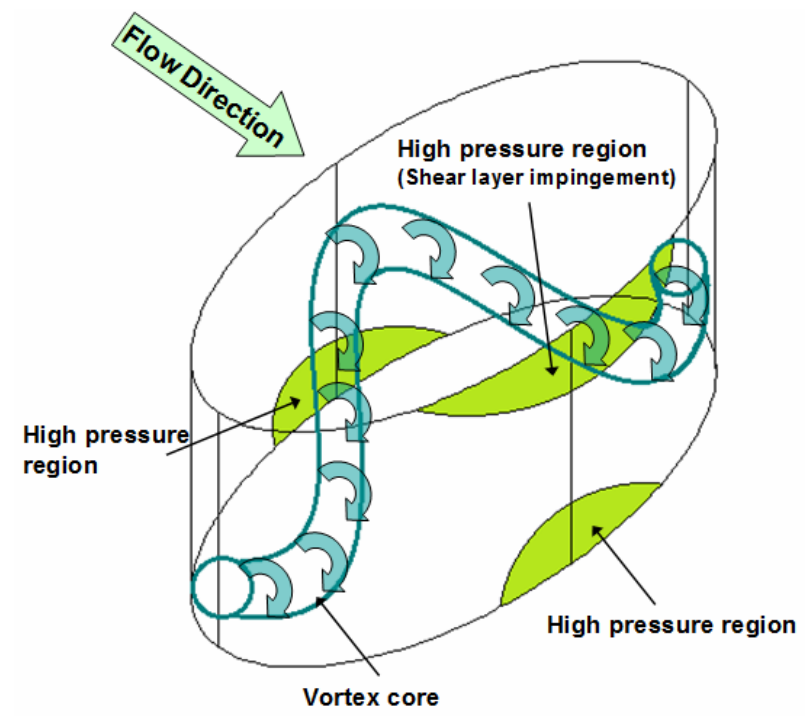

Figure 5-34 Schematic representation of the flow structure in an elliptical cavity in the symmetric-deep flow regime

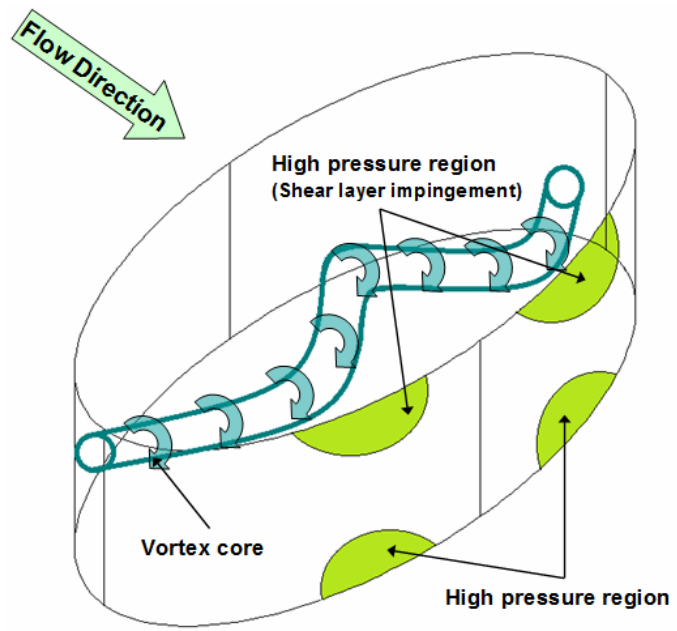

Figure 5-35 Schematic representation of the flow structure in an elliptical cavity in the symmetric-cellular structure flow regime

- For cavities with h/D ratios of 0.2 and 0.1 , the flow structure resembles that of shallow nominally two-dimensional cavities in the transitional flow regime, in which the shear layer is deflected into the cavity, but does not impinge onto the 
cavity base. The primary vortex core is parallel to the upstream wall of the cavity (Figure 5-36).

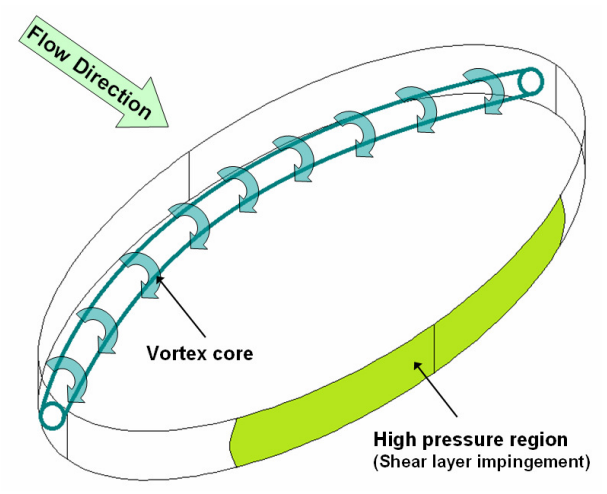

Figure 5-36 Schematic representation of the flow structure in an elliptical cavity in the symmetric-shallow flow regime

- The drag is found to be lower for cavities with larger h/D ratios (deeper cavities). This behaviour has been attributed to the increased entrainment of the shear layer in the case of shallow and transitional cavities.

- The flow is found to separate from the edge of the downstream wall of the cavity, to form a low pressure separation region. A region of velocity deficit is found to exist downstream of the cavity, for all depths. The velocity deficit for the cavity with $h / D=0.5$ is found to be larger those of $h / D=0.7$, which is consistent with the higher drag for this $\mathrm{h} / \mathrm{D}$ ratio.

- The flow in the cavity with $\mathrm{h} / \mathrm{D}=1.0$ is found to be more stable than those of cavities with $\mathrm{h} / \mathrm{D}=0.7$ and 0.5 . This is indicated by lower levels of fluctuating velocity components. The vortex inside the cavity is found to have a smaller timedependent displacement of the vortex core in the case of the deep cavity, which indicates that the higher level of velocity fluctuations is due to increased vortex oscillation. The vortex oscillations are possibly due to a flapping instability in the shear layer. 


\subsection{Nominally Two-Dimensional Flow Regime}

The nominally two-dimensional flow regime is associated with the cavity at $90^{\circ}$ yaw angle. The main feature of the flow in this regime is formation of a single, symmetric, vortex inside the cavity. In most cases, the vortex core is parallel to the minor axis of the cavity, and its position shows little variation due to three-dimensional effects. Figure 5-1 suggests that changes in depth of the cavity have little effects on the flow structure in this regime. In the following paragraphs, this behaviour will be investigated in detail based on the results of the present study. The investigation includes three $\mathrm{h} / \mathrm{D}$ ratios of 1.0, 0.5, and 0.1 , which represent deep, intermediate-depth, and shallow cavities, respectively. Various aspects of the flow, including shear layer behaviour, the three-dimensional flow structure, drag, the flow downstream of the cavity and the dynamic behaviour will be discussed and comparisons with previously published studies will be included where comparable results are available.

\subsubsection{Behaviour of the Shear Layer}

As shown before in sections 5.3 and 5.5, the behaviour of the separated shear layer, expressed in terms of the thickness of the shear layer and the position of the shear layer centre provides an insight into the flow structure in the cavity.

Variations of the vorticity thickness along the upper edge of the cavity $(\mathrm{y} / \mathrm{D}=0)$ in the cavity mid-plane $(\mathrm{z} / \mathrm{D}=0)$, which have been determined based on the results of numerical simulations, are shown for cavities with $\mathrm{h} / \mathrm{D}$ ratios of $0.1,0.5$, and 1.0 , in Figure 5-37. The figure indicates an almost linear growth, with a similar growth rate for the three $\mathrm{h} / \mathrm{D}$ ratios. The shear layer growth behaviour is similar to that observed in the case of elliptical cavities in symmetric flow regimes (yaw angle $=0^{\circ}$ ), shown in Figure 5-8, as well as the behaviour reported by Ashcroft and Zhang [23], Haigermoser et al. [24], Murray et al. [25], and Ukeiley and Murray [26] for nominally two-dimensional rectangular cavities exposed to turbulent boundary layers. Considering the different geometric proportions of the cavity in the mid-plane compared to the cavity with yaw angle $=0^{\circ}$, the above-mentioned observation further supports the conclusion that for a given set of upstream boundary layer conditions, the shear layer growth rate is uniform 
and independent of the geometric proportions of the cavity such as relative depth and length.

The quantitative value of the shear layer growth rate for the results shown in Figure 5-37 is $d \delta_{\omega} / d x=0.2$. This value is the same as the value, determined for the elliptical cavities in symmetric flow regimes (section 5.3), and the value of 0.19 , reported by Haigermoser et al. [24] for open rectangular cavities with $\mathrm{h} / \mathrm{D}=0.25$ exposed to a turbulent approaching flow with $\operatorname{Re}_{D}=\mathrm{O}\left(10^{4}\right)$.

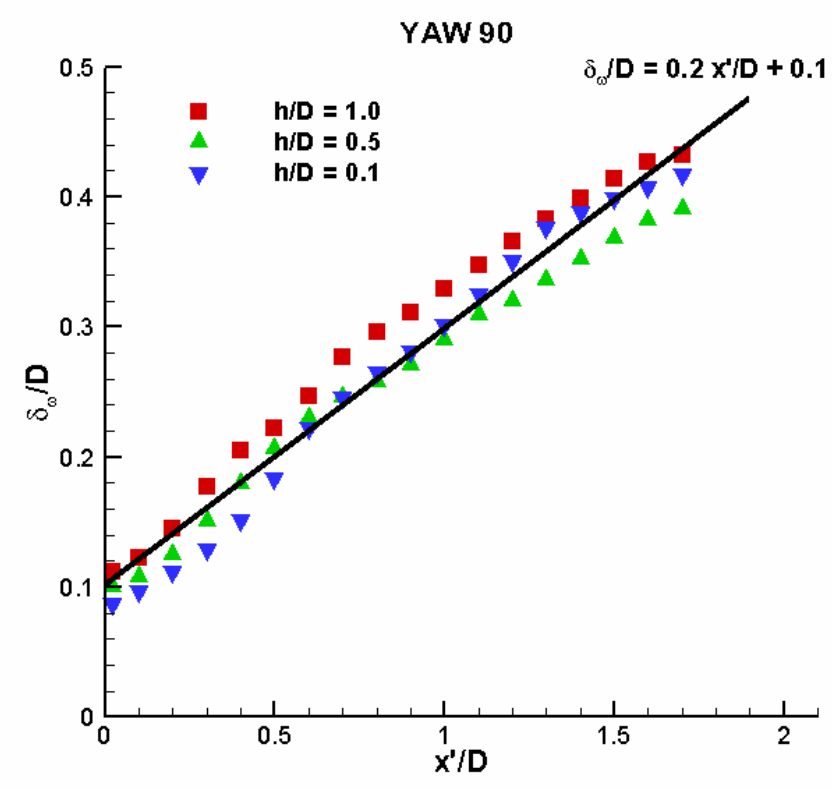

Figure 5-37 Shear layer thickness at $90^{\circ}$ yaw angle

The position of the centre of the shear layer relative to the upper edge of the cavity determined based on the results of numerical simulations are shown in the cavity midplane $(\mathrm{z} / \mathrm{D}=0)$, for cavities with $\mathrm{h} / \mathrm{D}=0.1,0.5$, and 1.0, in Figure 5-38.

The behaviour of the shear layer centre position for the cavities with $\mathrm{h} / \mathrm{D}=1.0$ and 0.5 is similar to that of the cavity with $\mathrm{h} / \mathrm{D}=1.0$ at yaw angle $=0^{\circ}$ (Figure 5-9), in which the shear layer extends over the upper edge of the cavity without considerable deflection into the cavity volume. Therefore, considering the uniform growth of the shear layer 
downstream of the separation point, the shear layer is expected to impinge onto the downstream wall. This behaviour suggests the existence of a flow structure similar to that of nominally two-dimensional rectangular cavities in the "open" flow regime, associated with deep cavities [18], for the elliptical cavities with $\mathrm{h} / \mathrm{D}=1.0$ and 0.5 .

The behaviour of the shear layer centre position for the cavity with $h / D=0.1$ is also found to be similar to that of the cavity with $\mathrm{h} / \mathrm{D}=0.1$ at yaw angle $=0^{\circ}$ (Figure 5-9), in which the shear layer is deflected into the cavity volume downstream of the separation point. Therefore, considering the uniform growth of the shear layer, the shear layer is expected to impinge onto, or approach the cavity base. This behaviour suggests the existence of a flow structure similar to that of nominally two-dimensional rectangular cavities in the "closed" or "transitional-closed" regimes associated with shallow cavities [18].

YAW 90

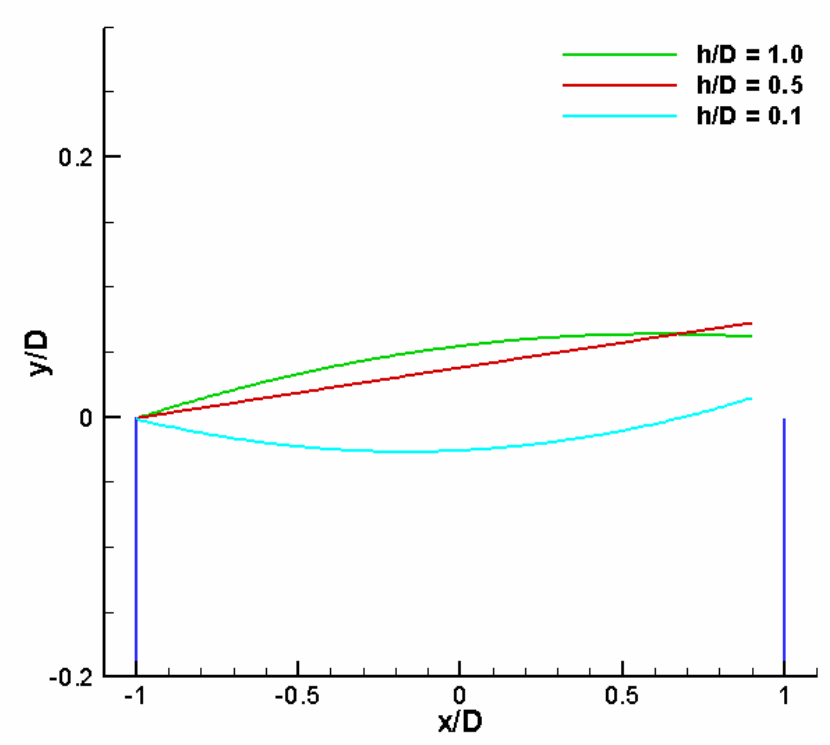

Figure 5-38 The location of shear layer centre at $90^{\circ}$ yaw angle

In general, the behaviour of the shear layer centre position for cavities with $h / D=1.0$ and 0.1 are found to be similar for the cavities in symmetric (yaw angle $=0^{\circ}$ ) and nominally two-dimensional (yaw angle $=90^{\circ}$ ) regimes. This behaviour, however, is different for 
$h / D=0.5$. For this $h / D$ ratio, a significant change in the behaviour of the shear layer, associated with the cellular flow structure, is observed for the cavity in the symmetric regime (sections 5.3). The shear layer for the cavity with $\mathrm{h} / \mathrm{D}=0.5$ at $90^{\circ}$ yaw angle, however, shows the typical behaviour of a nominally two-dimensional rectangular cavity in the "open" regime.

The behaviour of the shear layer depth will be related to the flow structure in the cavity in the following section, in which the flow structure for cavities with $\mathrm{h} / \mathrm{D}=0.1,0.5$, and 1.0 , at $90^{\circ}$ yaw angle will be investigated in detail.

\subsubsection{Flow Structure}

In the following paragraphs, a combination of the results of the present PIV velocity field measurements, numerical simulations, and flow visualizations, and the experiments by Hering [58] are used to identify the flow structure in elliptical cavities at $90^{\circ}$ yaw angle. Comparisons with previously published results are included where available.

\subsubsection{Deep cavity $(h / D=1.0)$}

Figure 5-39-(a) shows the vortex core position for the cavity with $\mathrm{h} / \mathrm{D}=1.0$. The cavity contains a single vortex which extends symmetrically between the two sidewalls, and shows little deformation due to three-dimensional effects. The vortex is slightly curved towards the downstream wall with the core position located at $\mathrm{x} / \mathrm{D}=0.05$ at $\mathrm{z} / \mathrm{D}=0.35$, and $x / D=0.2$ at $z / D=0$. The flow pattern is shown in more detail in two vertical planes located at $\mathrm{z} / \mathrm{D}=0$ and $\mathrm{z} / \mathrm{D}=0.35$, in Figure 5-40. The streamtraces indicate a flow pattern similar to that of a deep, nominally two-dimensional rectangular cavity containing a single captive vortex. An example of the flow pattern in a nominally two-dimensional rectangular cavity with a similar depth to streamwise length ratio, exposed to a turbulent upstream boundary layer [122] is shown in Figure 5-41 for comparison. However, the results of the present study show no trace of a secondary vortex similar to that observed in Figure 5-41. 


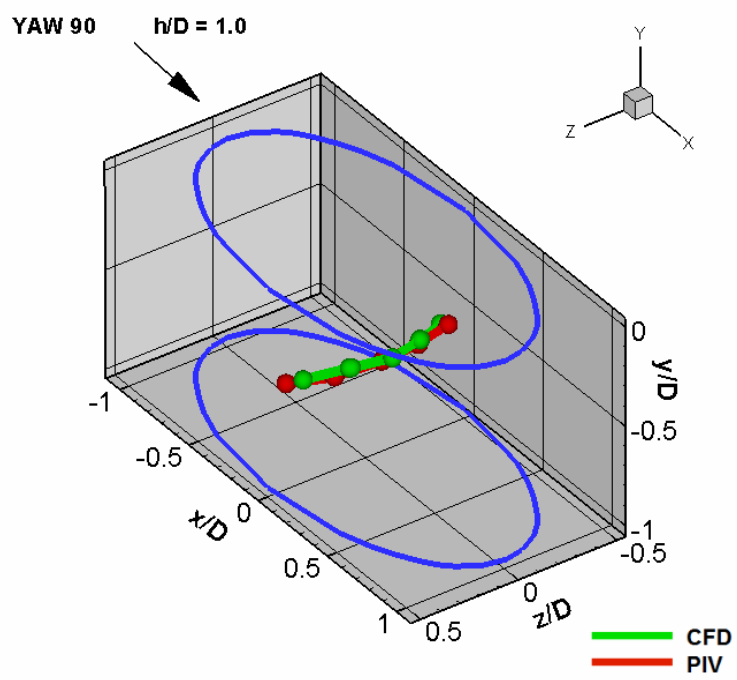

(a)

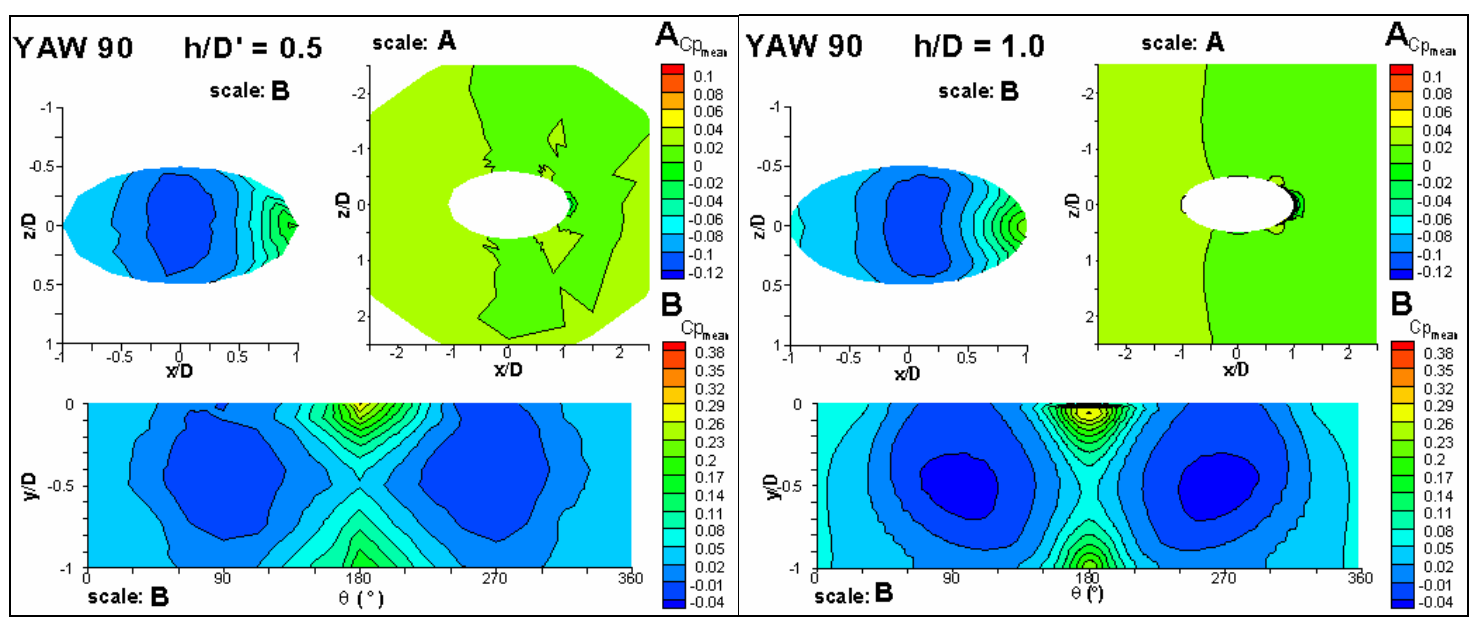

(b)

(c)

Figure 5-39 Vortex core position (a) and pressure coefficient contours on cavity base, ground plane, and side walls for $h / D=1.0$, yaw angle $=90^{\circ}$ from reference [58] (b) and numerical simulations (c)

Figure 5-42 shows an instantaneous image of the present helium bubble flow visualizations, for the cavity with $\mathrm{h} / \mathrm{D}=1.0$ at $90^{\circ}$ yaw angle. The figure verifies the existence of a captive vortex, with its core located at $\mathrm{x} / \mathrm{D}=0.1$.

Similar to the previous cases studied in this chapter, the pressure coefficients contours based on numerical simulations are in accordance with the flow structure (Figure 5-39(c)). The low pressure regions on the cavity base (between $\mathrm{x} / \mathrm{D}=0$ and 0.25 ) and sidewalls (at angular positions of $90^{\circ}$ and $270^{\circ}$ ) correspond to the position of the vortex 
core relative to the cavity base and sidewalls. Also, a symmetric high pressure region can be observed on the downstream wall around the angular position of $180^{\circ}$. This region corresponds to the location where the shear layer impinges upon the downstream cavity wall after bridging the cavity, as shown in Figure 5-40-(a).

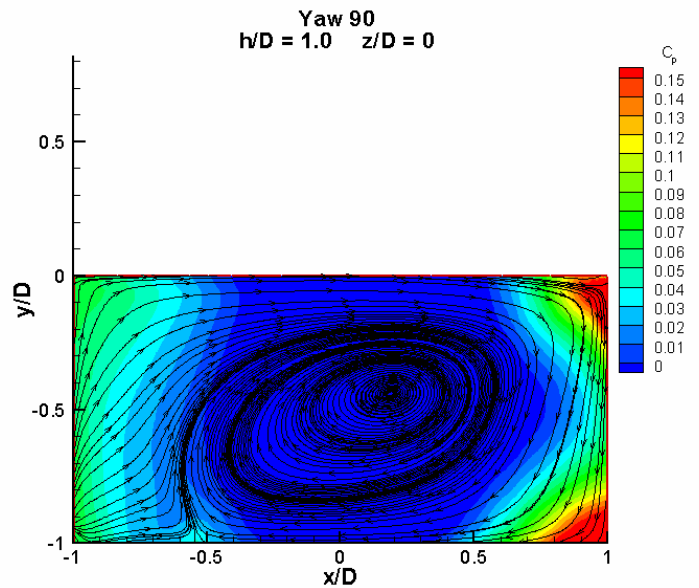

(a)

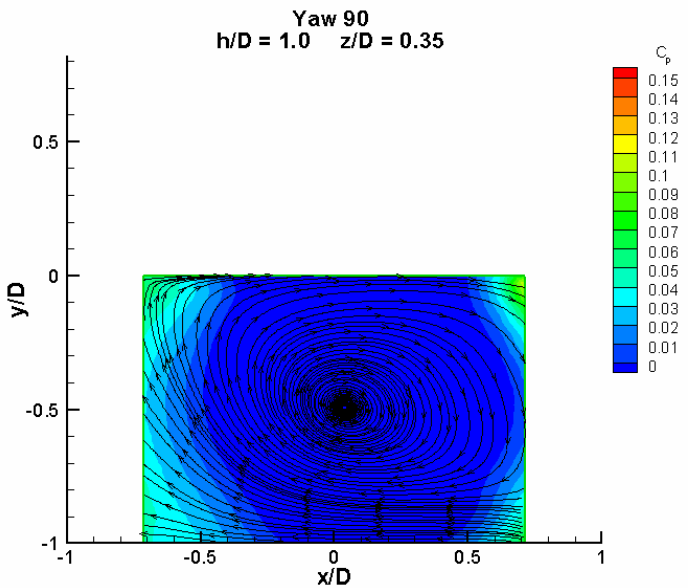

(b)

Figure 5-40 Sectional streamlines and $C_{p}$ contours for cavity with $\mathrm{h} / \mathrm{D}=1.0$ at $90^{\circ}$ yaw angle at $\mathrm{z} / \mathrm{D}=0$ (a) and $\mathrm{z} / \mathrm{D}=\mathbf{0 . 3 5}(\mathrm{b})$

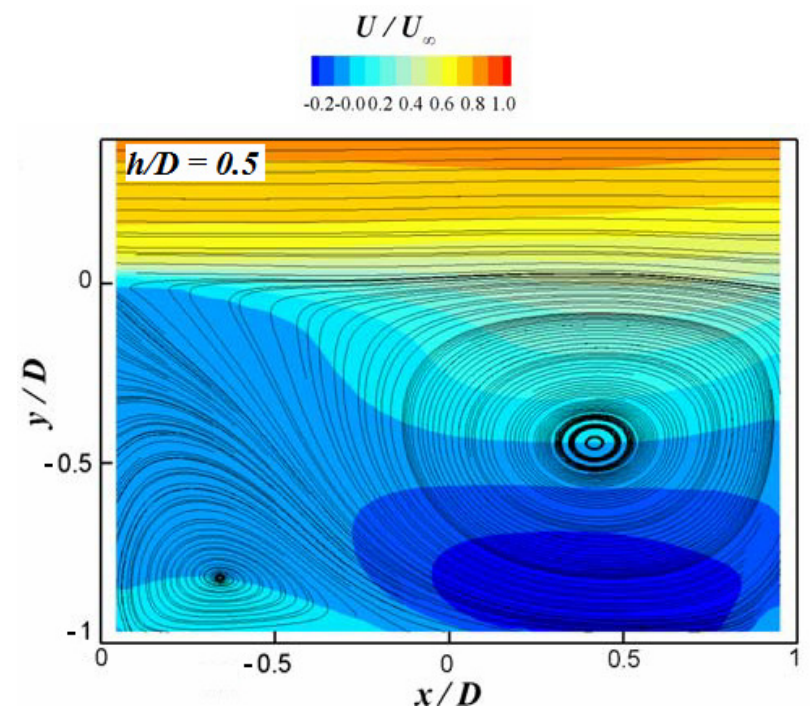

Figure 5-41 Example of mean velocity contours and streamlines in a nominally twodimensional rectangular cavity with $h / D=0.5$ (adapted from ref. [122]) 
The pressure coefficient contours based on the experiments by Hering [58] (Figure 5-39(b)), indicate similar patterns which suggest a similar vortex structure, considering the correspondence between the surface pressure distributions and the position of the vortex (discussed in section 5.2).

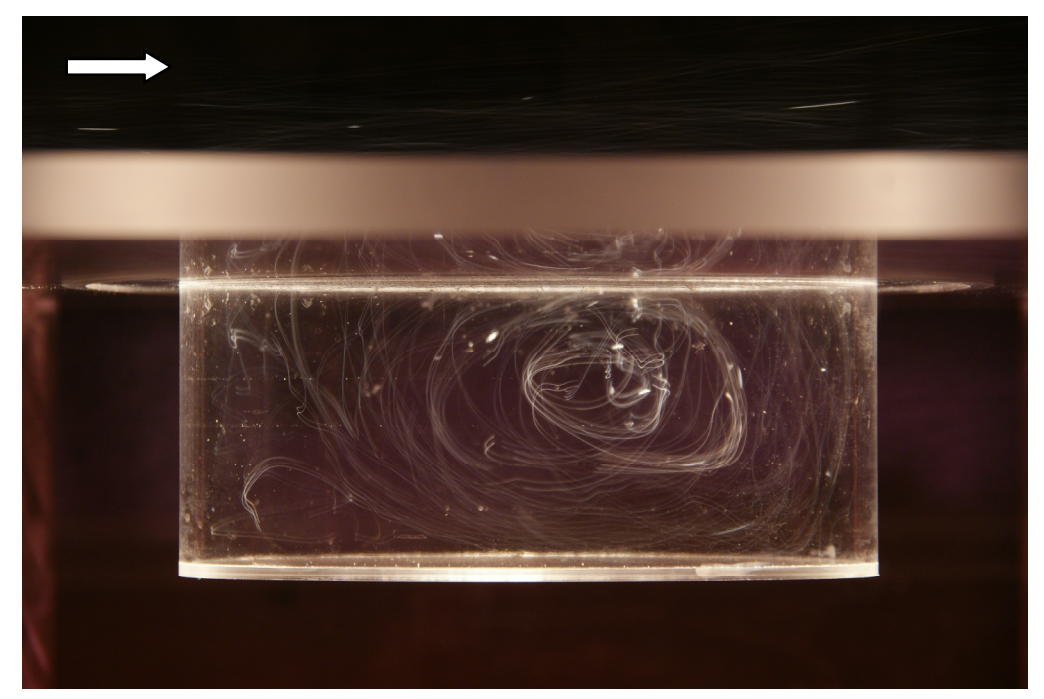

Figure 5-42 Instantaneous flow visualization images obtained using the Helium bubble technique, for the cavity with $\mathrm{h} / \mathrm{D}=1.0$ at yaw angle $=90^{\circ}$

\subsubsection{Intermediate depth cavity $(\mathrm{h} / \mathrm{D}=0.5)$}

Figure 5-7 shows the vortex core positions for the cavity with $\mathrm{h} / \mathrm{D}=0.5$. The main vortex inside the cavity extends symmetrically between the two sidewalls, at $\mathrm{x} / \mathrm{D}=0.3$, and shows little deformation due to three-dimensional effects. The feature that distinguishes the flow structure in this case from that of the deep cavity is the existence of a secondary vortex parallel to the primary vortex, with an opposite sense of rotation. The secondary vortex is located at $x / D=-0.2$. The streamtraces in vertical planes located at $x / D=0$ and 0.35, which are shown in Figure 5-43 confirm the existence of a small secondary vortex. The secondary vortex is very close to the base of the cavity, and cannot be observed in the results of the PIV measurements due to reflections from the cavity base, except for the mid-plane $(\mathrm{z} / \mathrm{D}=0)$, where the vortex is slightly higher. 


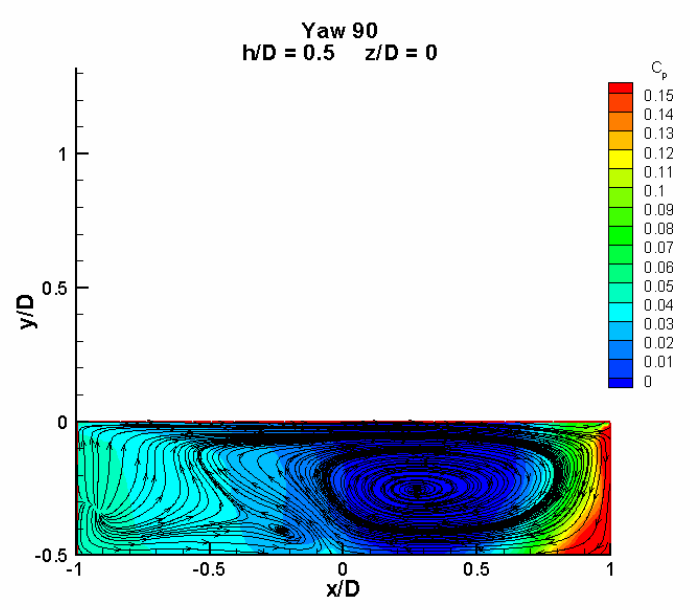

(a)

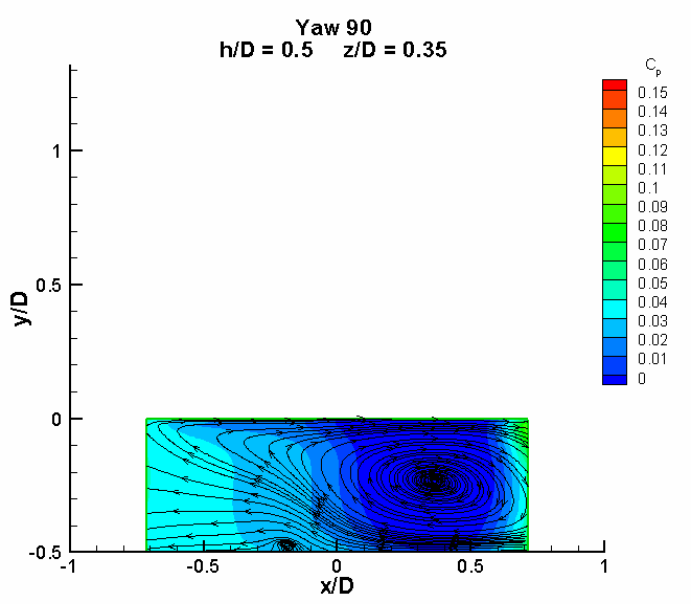

(b)

Figure 5-43 Streamlines for cavity with $\mathrm{h} / \mathrm{D}=0.5$ at $90^{\circ}$ yaw angle at $\mathrm{z} / \mathrm{D}=0$ (a) and $\mathrm{z} / \mathrm{D}=0.35$ (b)

A comparison between the flow structure in this case, and that of a nominally twodimensional rectangular cavities with similar depth to streamwise length ratio [24] shows that the general flow pattern is similar for both cases. However, the secondary vortex is located further downstream in the case of the elliptical cavity (Figure 5-44). It should be noted that in the case of open cavities with a large primary vortex, the secondary vortices are driven by the primary vortex and are, therefore, expected to be parallel to it. In the case of a nominally two-dimensional rectangular cavity, this means that the secondary vortex is also parallel to the upstream wall. In the case of the elliptical cavity at $90^{\circ}$ yaw angle, the upstream wall is highly curved, therefore, the secondary vortex driven by the straight primary vortex cannot be expected to form parallel to the upstream wall. This explains the formation of the secondary vortex at a position further downstream, compared to nominally two-dimensional rectangular cavities.

The pressure coefficient contours obtained based on numerical simulations (Figure 5-7(c)) show the effect of the primary vortex as a low-pressure region on the cavity base (x/D between 0 and 0.5 ), and the sidewalls (angular positions of $135^{\circ}$ and $225^{\circ}$, approximately). Again, a symmetric high pressure region corresponding to the location of shear layer impingement can be observed on the downstream wall around the angular position of $180^{\circ}$. The pressure coefficient contours based on the experiments by Hering 
[58] show a similar pattern, suggesting a similar flow structure (Figure 5-7-(b)). No trace of the smaller secondary vortex can be observed in the pressure coefficients distributions based on the present numerical simulations and Hering's experiments.

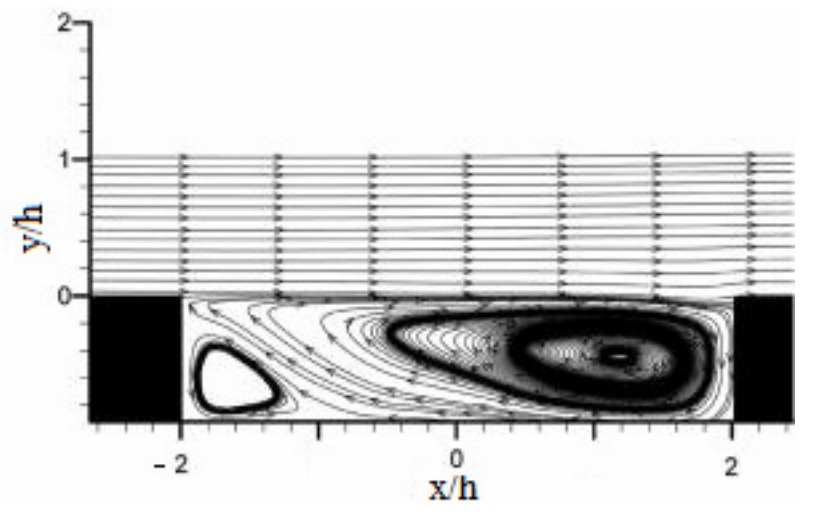

Figure 5-44 streamlines of a nominally two-dimensional rectangular cavity $(\mathrm{h} / \mathrm{D}=\mathbf{0 . 2 5})$ (adapted from ref. [24])

Figure 5-45 shows the pressure coefficient contours in a rectangular cavity with W/D $=2$ and $\mathrm{h} / \mathrm{D}=0.25$, at $90^{\circ}$ yaw angle. The figure indicates a pressure distribution very similar to that of the present study, in which low-pressure areas corresponding to a straight vortex can be observed on the cavity base and sidewalls, at a streamwise position of approximately $0.2 \mathrm{x} / \mathrm{D}$.

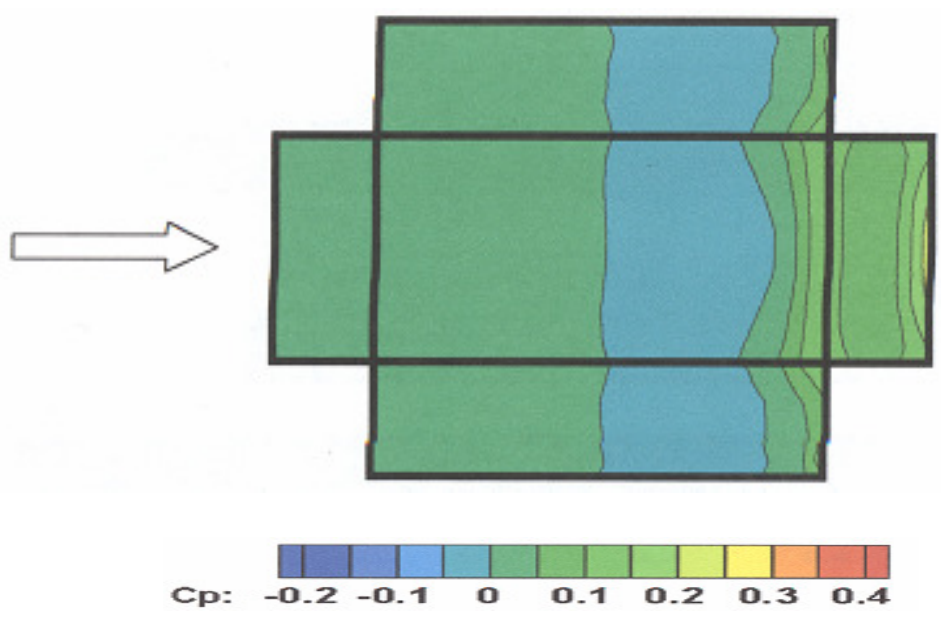

Figure 5-45 $C_{p}$ contours for a rectangular cavity $(W / D=2 \&$ $\mathrm{h} / \mathrm{D}=0.5$ ) at $0^{\circ}$ yaw angle (adapted from ref. [49]) 


\subsubsection{Shallow cavity $(\mathrm{h} / \mathrm{D}=\mathbf{0 . 1})$}

Figure 5-46-(a) shows the vortex core position for the cavity with $\mathrm{h} / \mathrm{D}=0.1$. The vortex core position indicates formation of a recirculation region downstream of the upstream wall, due to separation of the shear layer from the upstream lip of the cavity wall. Similar to the cavity with $\mathrm{h} / \mathrm{D}=0.1$ at $0^{\circ}$ yaw angle (section 5.3.2), the vortex core is found to be parallel to the upstream wall and, therefore, highly curved. The pressure coefficient distributions, shown in Figure 5-46-(c), confirm the formation of the recirculation zone near the upstream wall. The pressure coefficient contours based on the experiments by Hering [58] are also shown in Figure 5-46-(b). A comparison between Figure 5-46-(b) and (c) indicates similar patterns in pressure distributions, suggesting a similar flow structure.

No trace of a secondary recirculation zone is found further downstream, according to the vortex core positions and pressure distributions. However, the pressure increases on the cavity base, towards the downstream wall. This is followed by a low-pressure region on the boundary layer plate, immediately downstream of the downstream edge of the cavity wall. This pressure distribution indicates that although the shear layer does not impinge onto the cavity base, it enters the cavity volume and approaches the base, and then leaves the cavity volume, forming a separation region at the edge of the downstream cavity wall.

The streamtraces in the vertical planes located at $\mathrm{z} / \mathrm{D}=0$ and 0.35 , shown in Figure 5-47, confirm the above-mentioned flow structure. The recirculation zone, the entrainment of the shear layer, and the high-pressure region associated with shear layer impingement onto the downstream wall are clearly visible in the figures. The streamtraces in a twodimensional rectangular cavity exposed to a turbulent upstream boundary layer [123] are shown in Figure 5-48 for comparison. The depth to streamwise length ratio of the cavity in Figure 5-48, which was the shallowest one for which the flow pattern was reported in the literature, is equivalent to that of an elliptical cavity with $\mathrm{h} / \mathrm{D}=0.2$ at $90^{\circ}$ yaw angle. Although the size of the recirculaton zone is different from that of the present study, due to different upstream boundary layer conditions and freestream velocity, the flow structure is qualitatively similar to that observed in Figure 5-47, which further confirms the similarities between the flow structure in elliptical cavities at yaw angle $=90^{\circ}$, and 
that of nominally two-dimensional rectangular cavities. It should be noted that the depth to streamwise length ratio of the cavity in Figure 5-48, which was the shallowest for which the flow pattern was reported in the literature, is equivalent to that of an elliptical cavity with $\mathrm{h} / \mathrm{D}=0.2$ at $90^{\circ}$ yaw angle.

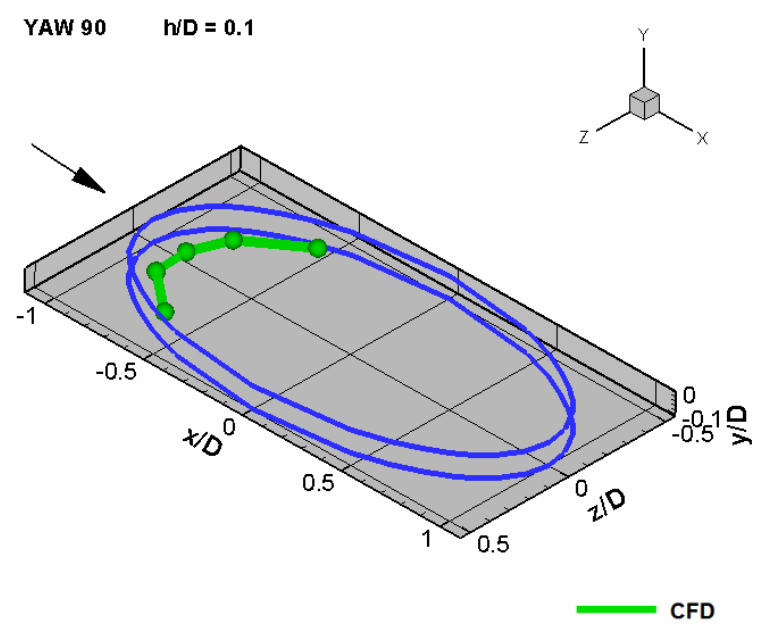

(a)

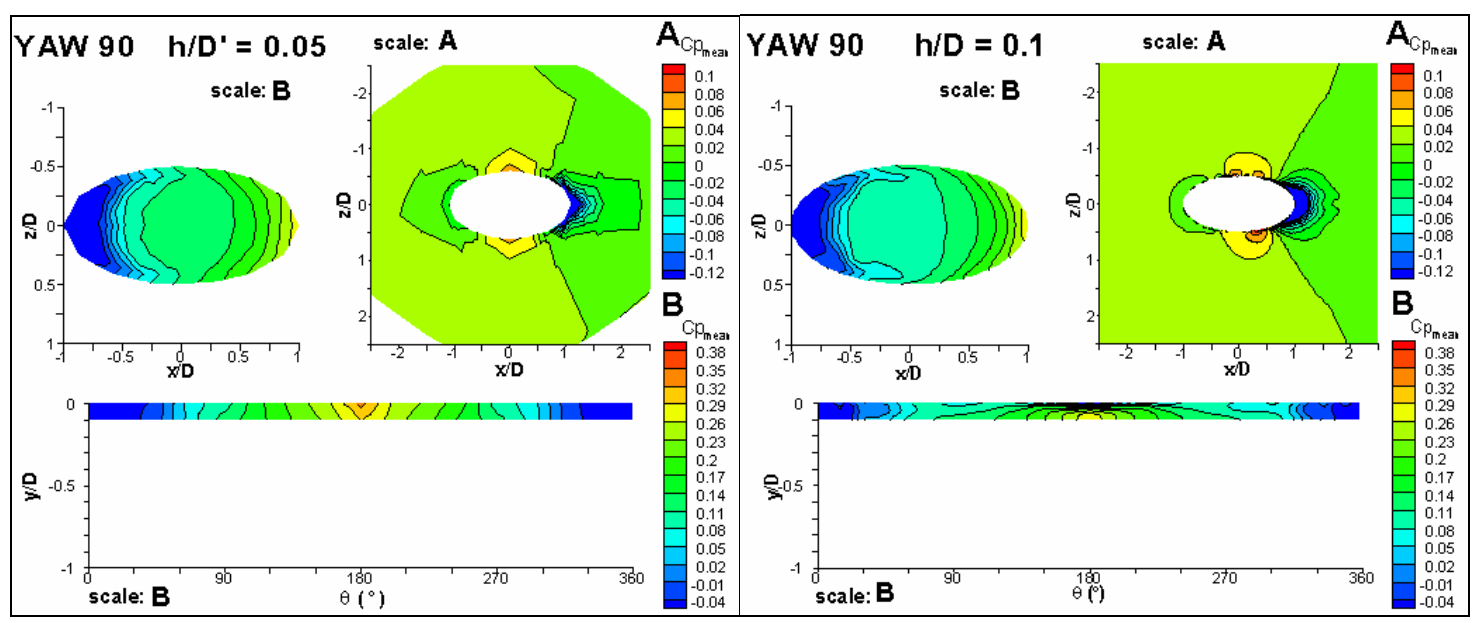

(b)

(c)

Figure 5-46 Vortex core position (a) and pressure coefficient contours on cavity base, ground plane, and side walls for $\mathrm{h} / \mathrm{D}=\mathbf{0 . 1}$, yaw angle $=\mathbf{9 0}^{\circ}$ from reference [58] (b) and numerical simulations (c)

The results presented in the previous paragraphs indicate the limited three-dimensional effects in the vortex in the cavity with $90^{\circ}$ yaw angle, and the associated pressure 
distribution on the cavity base. This behaviour, which indicates the similarity between the flow structure of the cavity with $90^{\circ}$ yaw angle with that of a nominally two-dimensional rectangular cavity, makes it possible to compare the trends in centreline pressure distribution for the two cases, and extend the classification of the flow regimes of a nominally two-dimensional cavity to the cavity with $90^{\circ}$ yaw angle. Variations of pressure coefficient $\left(C_{p}\right)$ along the centreline of the cavity, obtained based on the results of the numerical simulations, are shown in Figure 5-49-(a). Figure 5-49-(b) shows the results of the experiments by Hering [58], which show qualitatively similar profiles (section 3.7). A comparison of Figure 5-49-(a) with the classification of the flow regimes in nominally two-dimensional cavities and their associated pressure distributions (Figure $2-4$ ) indicates that the pressure distribution for the cavity with $h / D=0.1$ follows the trend reported by Plentovich [15] for the cavities in the "closed" flow regime $(\mathrm{h} / \mathrm{D}<0.16)$. The pressure distributions for cavities with $\mathrm{h} / \mathrm{D}=0.2$ and 0.35 follow the trend associated by Plentovich to cavities in the "transitional" flow regime $(\mathrm{h} / \mathrm{D}>0.2)$. Finally, the cavities with $\mathrm{h} / \mathrm{D}=0.5,0.7$, and 1.0 show pressure distributions similar to that associated with the cavities in the "open" flow regime (h/D > 0.5) by Plentovich [13]. It can be concluded that the flow regimes in elliptical cavities at $90^{\circ}$ yaw angle conform to the flow regimes with nominally two-dimensional rectangular cavities of similar h/D ratio.

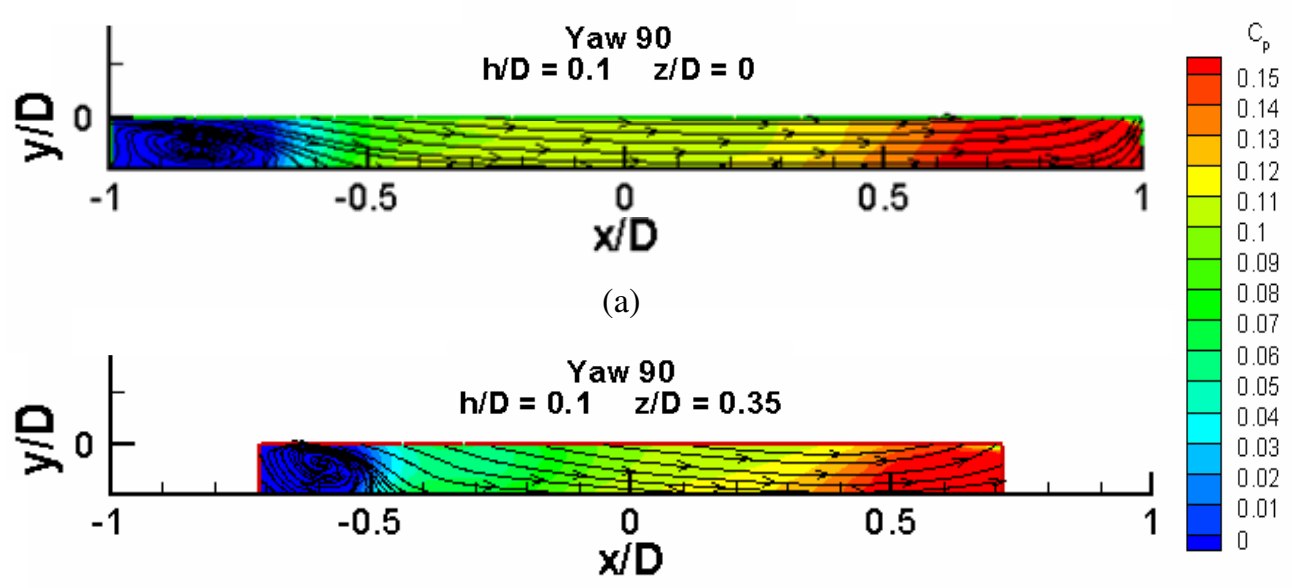

(b)

Figure 5-47 Streamlines for cavity with $\mathrm{h} / \mathrm{D}=0.1$ at $90^{\circ}$ yaw angle at $\mathrm{z} / \mathrm{D}=0$ (a) and $\mathrm{z} / \mathrm{D}=0.35$ (b) 


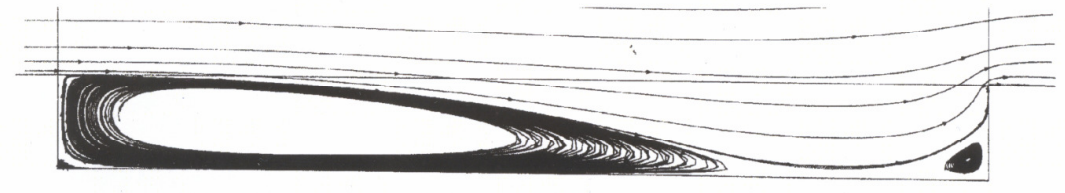

Figure 5-48 Two-dimensional RANS computation of the flow pattern for $h / D=0.1[123]$

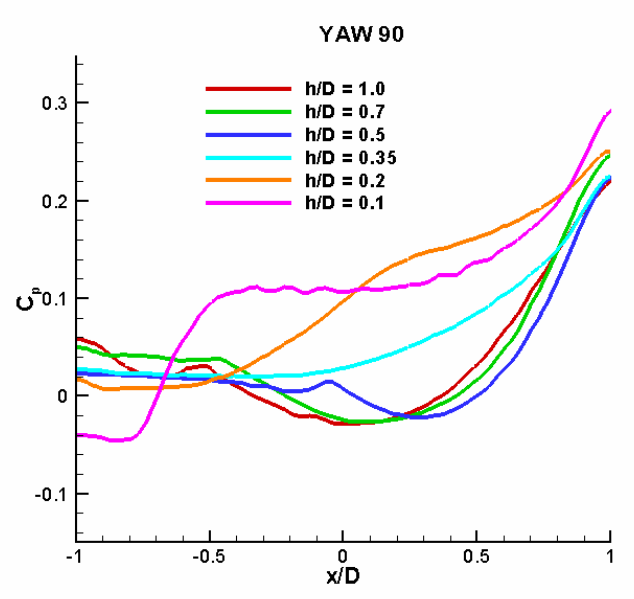

(a)

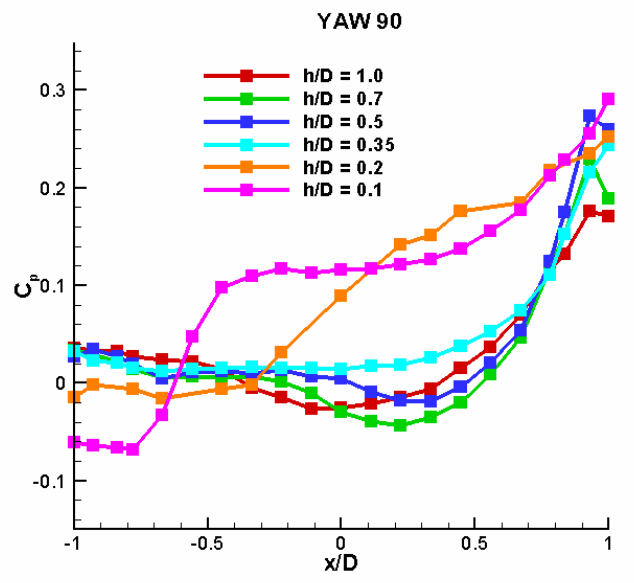

(b)

Figure 5-49 Comparison of $C_{p}$ profiles on the centreline of the cavity base at yaw angle $=90^{\circ}$ from numerical simulations (a) and reference [58] (b)

\subsubsection{Drag Characteristics}

The normalized drag coefficients $\left(\frac{\Delta C_{D}}{c_{f}}\right)$ of elliptical cavities with $\mathrm{h} / \mathrm{D}$ ratios between 0.1 and 1.0 at $90^{\circ}$ yaw angle, obtained based on the present numerical simulations, are presented in Figure 5-50. These results are compared with the previously published drag data for elliptical and rectangular cavities with $\mathrm{W} / \mathrm{D}=2$ at $90^{\circ}$ yaw angle [5], [48] and [58], as well circular cavities [52] and [53].

The results of the present study are within the uncertainty limit of the values reported by Hering [58], and close to the values reported by Savory and Toy [18]. The general trend observed in Figure 5-50 is similar to that previously described in section 5.3.3 in the case of cavities with $0^{\circ}$ yaw angle, in which lower drag coefficients are observed for cavities with larger $\mathrm{h} / \mathrm{D}$ ratios. The larger drag for the shallower cavity has been attributed to the increased entrainment of the shear layer compared to deep cavities, which results in a 
larger high pressure region on the downstream wall [48]. A similar trend can be observed in the present results for cavities at $90^{\circ}$ yaw angle, in which the lowest position of the shear layer centre, and the largest high-pressure region is observed for the cavity with $\mathrm{h} / \mathrm{D}=0.1$, in Figure 5-38 and Figure 5-46, respectively.

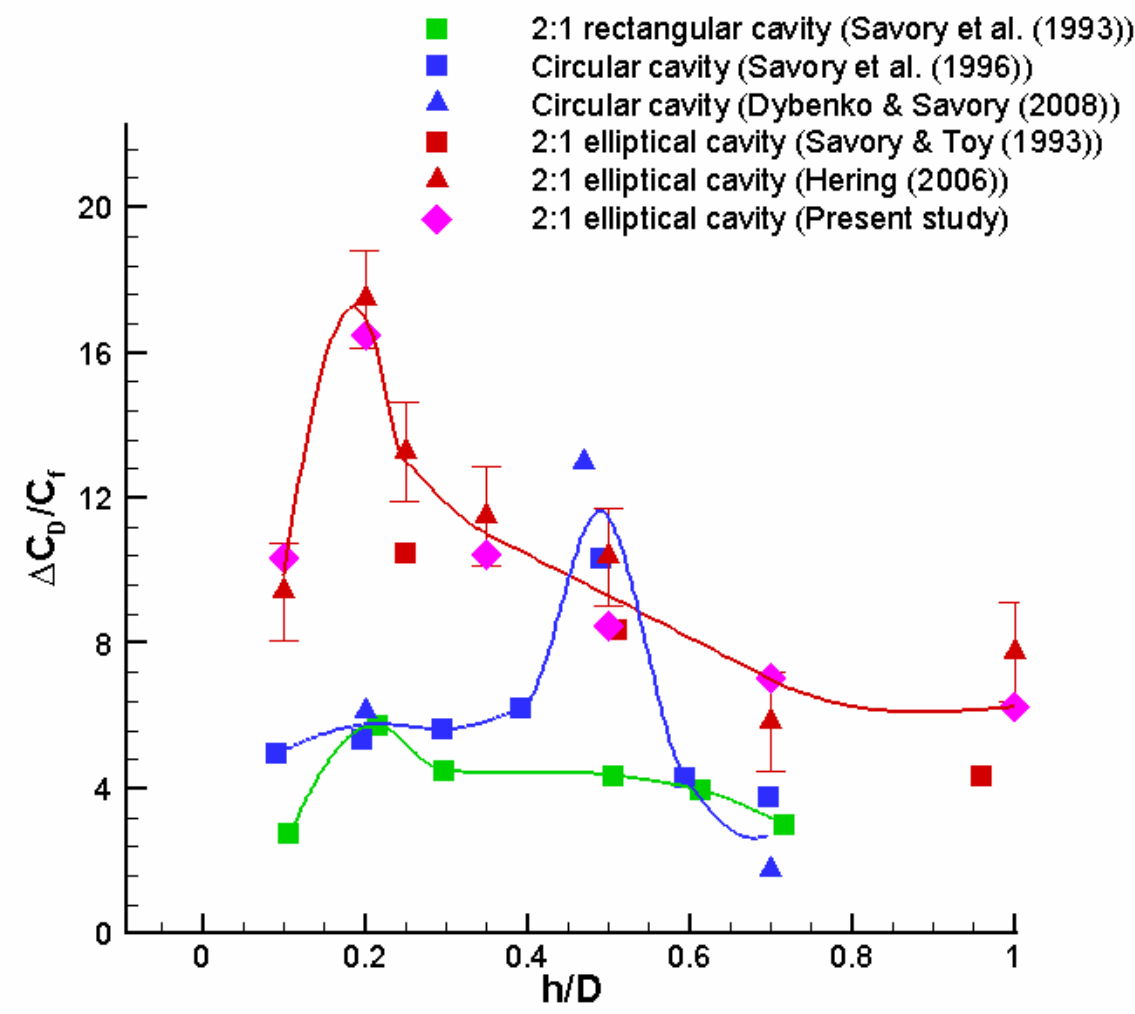

Figure 5-50 Comparison of drag coefficient at yaw angle $=90^{\circ}$ (The lines are added to visualize the general trend for each planform shape.)

\subsubsection{Flow Downstream of the Cavity}

As mentioned in Chapter 2, for rectangular cavities with large length to width ratio, flow is drawn into the cavity over the edges of the sidewalls. This behaviour, which is due to the effect of the shear layer being entrained into the cavity following separation from the edge of the upstream wall, leads to formation of "side edge vortices" along the edges of the side walls. These vortices extend in the streamwise direction, and continue into the flow downstream of the cavity [14]. 
The results of the present study indicate that the flow downstream of the elliptical cavity with a $90^{\circ}$ yaw angle also contains streamwise vortices. However, as will be shown in this section, the mechanism of their formation is different from that described in [14]. Due to this difference, the classical definition of "side edge vortices" is not valid for these vortices. Formation of the streamwise vortices and their effect on the flow downstream of the cavity is studied in this section for $\mathrm{h} / \mathrm{D}$ ratios of $0.7,0.5$, and 0.2 , based on velocity and vorticity distributions in horizontal and vertical planes at various locations downstream of the cavity. The results reported by Hering [58] for elliptical cavities with similar $\mathrm{h} / \mathrm{D}$ ratios are also presented for comparison.

Figure 5-51 shows distributions of velocity deficit $\left(\frac{\Delta \bar{u}}{U_{\text {inf }}}\right)$, which is defined in Equation 51 , in vertical planes located at $x / D=0.1$ and $x / D=2.6$, based on the results of the numerical simulations of the present study, and the experiments by Hering [58]. For all three $h / D$ ratios, the results reported by Hering [58] for the plane located at $x / D=0.1$ show two regions of velocity deficit located symmetrically on the two sides of the cavity centreline $(\mathrm{z} / \mathrm{D}= \pm 0.5)$, which correspond to the streamwise vortices. The results of the present numerical simulations show similar low velocity regions for cavities with $h / D=$ 0.7 and 0.2 . However, for $\mathrm{h} / \mathrm{D}=0.5$ a single low velocity region appears in the results of the numerical simulations at $\mathrm{x} / \mathrm{D}=2.6$.

The results of the present PIV measurements also indicate evidence of the streamwise vortices. Figure 5-52 shows the velocity distribution downstream of cavities with of 0.7 and 0.5 at $90^{\circ}$ yaw angle, based on PIV measurements in a horizontal plane located $8 \mathrm{~mm}$ above the upper edge of the cavity ( $\mathrm{y} / \mathrm{D}=0.08$ ). Evidence of the vortices for both $\mathrm{h} / \mathrm{D}$ ratios can be observed in Figure 5-52 which shows two low velocity regions extending downstream of the cavity. The low velocity regions are located at the spanwise positions of $\mathrm{z} / \mathrm{D}= \pm 0.5$, which correspond to the spanwise extremes of the cavity sidewalls. Considering the uniform spanwise velocity distribution upstream of the cavity (section 4.4), the reason for the slightly asymmetric velocity deficit about the centreline for both cases is presumably a small error in the setting of the yaw angle to $90^{\circ}$ in the experiment, or a slight non-uniformity in the cavity model. 


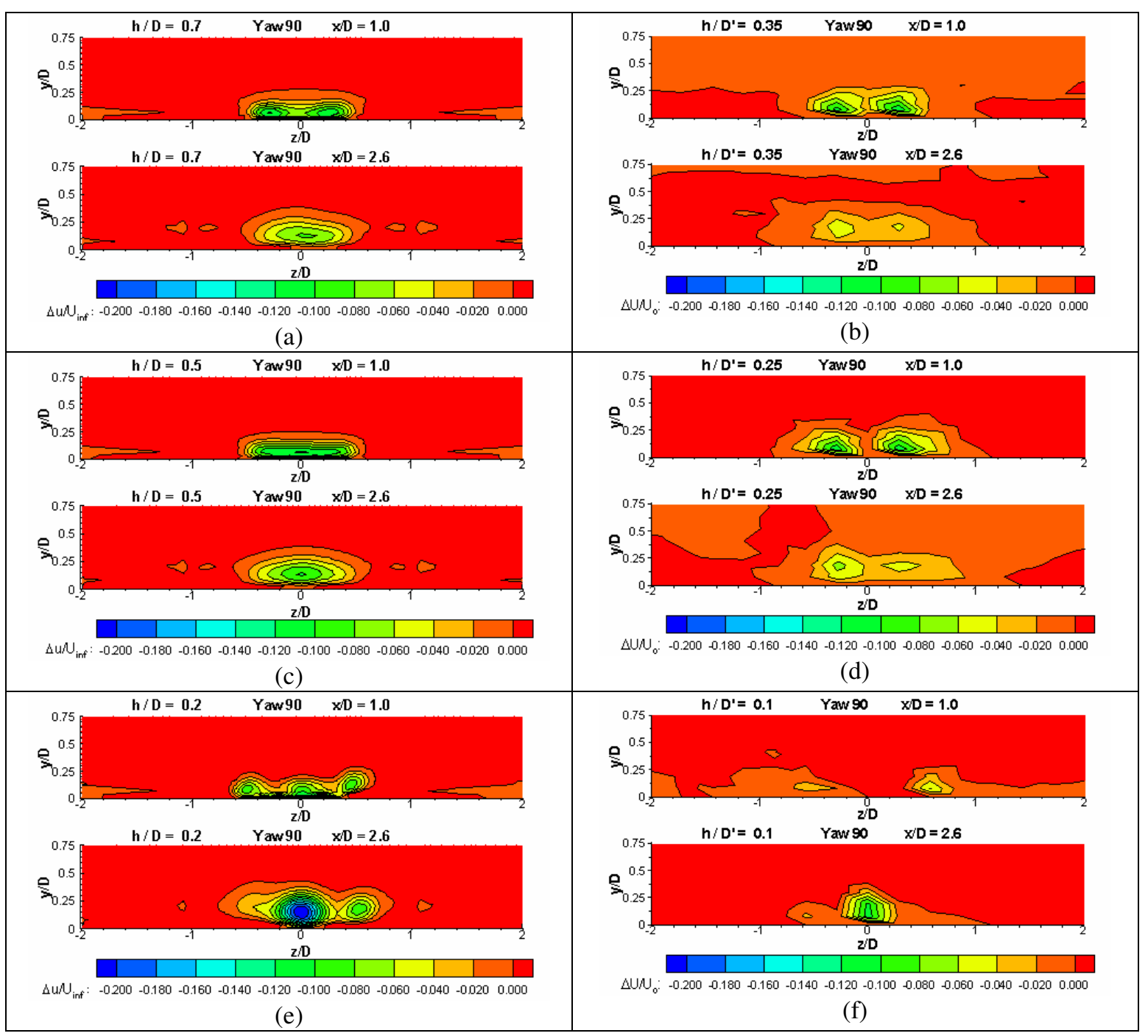

Figure 5-51 Velocity deficit contours based on numerical simulations

(left) and experimental measurements [58] (right) at $90^{\circ}$ yaw angle

A quantitative comparison of the spanwise variations of $\frac{\Delta \bar{u}}{U_{\mathrm{inf}}}$ based on the results of numerical simulations and PIV measurements is presented in Figure 5-53 for cavities with $\mathrm{h} / \mathrm{D}=0.7$ and 0.5 . The results of the PIV measurements, extracted at $\mathrm{x} / \mathrm{D}=1.5$, show two low velocity regions at the approximate spanwise positions of $\mathrm{z} / \mathrm{D}= \pm 0.5$. The plot of velocity variations extracted from the results of the numerical simulations at $\mathrm{x} / \mathrm{D}=$ 1.0 shows two low velocity regions at the same spanwise locations. At $x / D=1.5$, the results of the present PIV measurements and the experiments by Hering [58] indicate two distinct low velocity regions. However, the results of the numerical simulations show a 
single low velocity region at this downstream position, indicating that the numerical simulations have not been able to predict the flow in this region properly due to the approximations involved in the simulation.

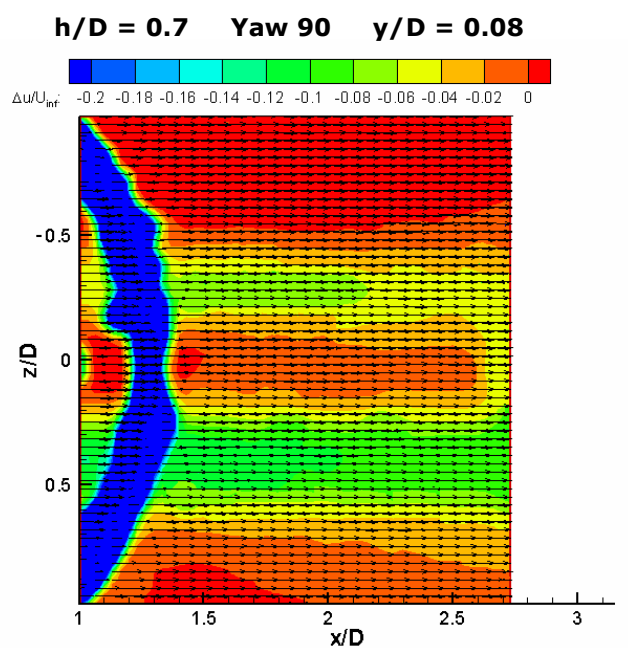

(a)

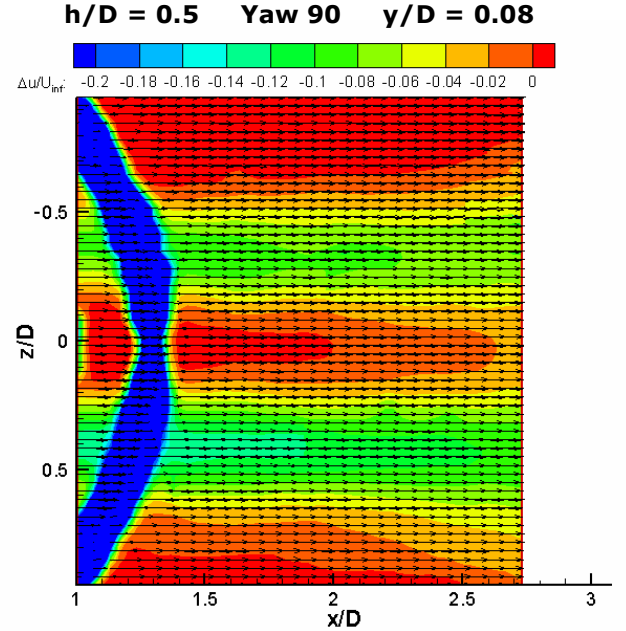

(b)

Figure 5-52 Velocity deficit contours based on PIV measurements in a horizontal plane at $90^{\circ}$ yaw angle

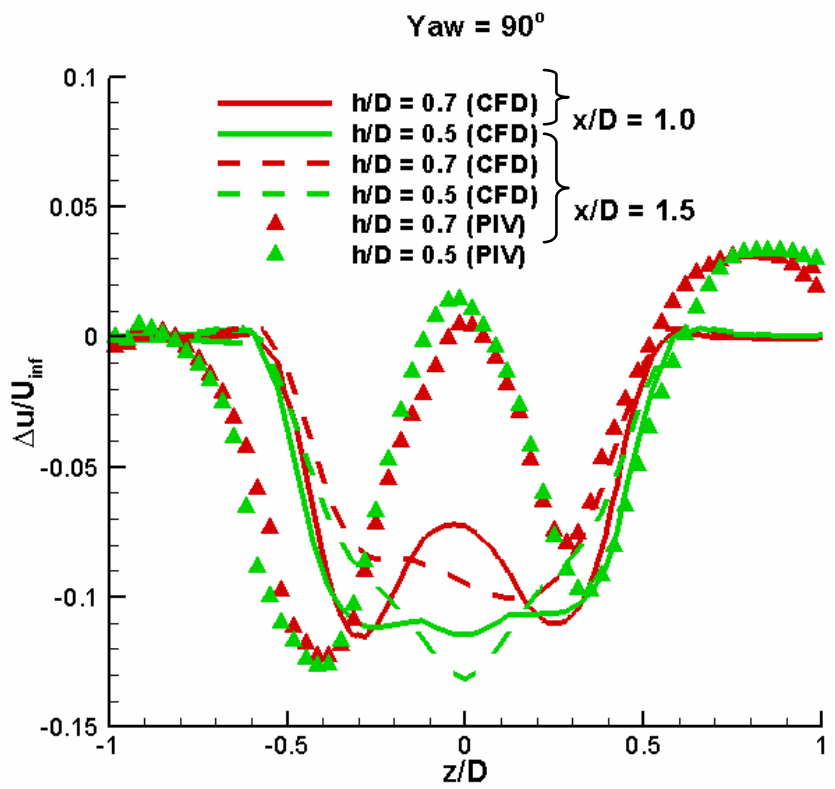

Figure 5-53 Quantitative comparison of velocity deficit at $90^{\circ}$ yaw angle 
An investigation of the distribution of streamwise vorticity $\left(\omega_{x}\right)$ in the vicinity of the cavity further confirms that the low velocity regions observed in the above-mentioned results are associated with the streamwise vortices. Such an investigation is only possible based on the results of the numerical simulations, which provide data for all three velocity components. Figure 5-54 shows distribution of streamwise vorticity in a vertical plane located at $x / D=1.0$, for cavities with $h / D=0.7$ and 0.2 . For both $h / D$ ratios, the figure shows two regions of streamwise vorticity of opposite sign, in spanwise locations which are consistent with the low velocity regions in the wake velocity deficit contours shown in Figure 5-51. It should be noted that positive values of $\omega_{x}$ indicate streamwise vorticity in the downstream (positive-x) direction, with circulation in clockwise direction looking downstream.
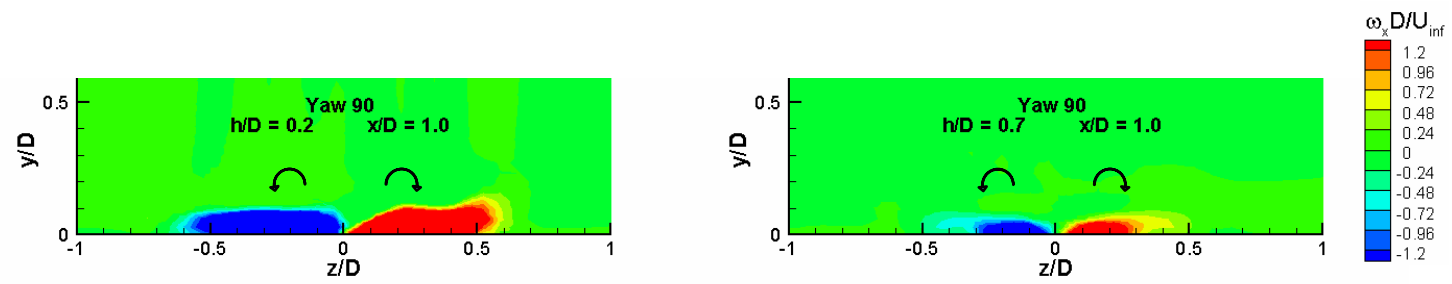

Figure 5-54 Streamwise vorticity contours at $\mathrm{x} / \mathrm{D}=1.0$ for $\mathrm{h} / \mathrm{D}=0.7$ (a) and $\mathrm{h} / \mathrm{D}=0.5$ (b) at $90^{\circ}$ yaw angle

This confirms that the velocity deficit in these cases is due to the streamwise. The origin of the vortices is best visualized by Figure 5-55, which shows the distribution of streamwise vorticity in a horizontal plane located at $y / D=0.08$, for the cavity with $h / D$ $=0.7$. The figure shows two regions of streamwise vorticity of opposite sign emerging immediately downstream of the two spanwise extremes of the cavity, which extend into the flow downstream of the cavity.

These regions of streamwise vorticity are caused by separation of the flow from the downstream wall of the cavity, which is accompanied by formation of a recirculation region immediately downstream of the edge of the downstream wall, as discussed in section 5.3.4. The recirculation region is parallel to the edge of the downstream wall. Since the edge is curved in the streamwise direction, the vorticity in the recirculation 
zone also has a streamwise vorticity component, which is visible in Figure 5-55. This mechanism of formation of the side edge vortices is completely different from the mechanism described in reference [14] and shown in Figure 2-5-(a) for cavities with rectangular planforms. Formation of the vortices in the case of the elliptical cavity is due to the flow exiting the cavity rather than that being drawn into the cavity. As a result, the sense of rotation of the vortices is reversed compared to those associated with rectangular cavities.

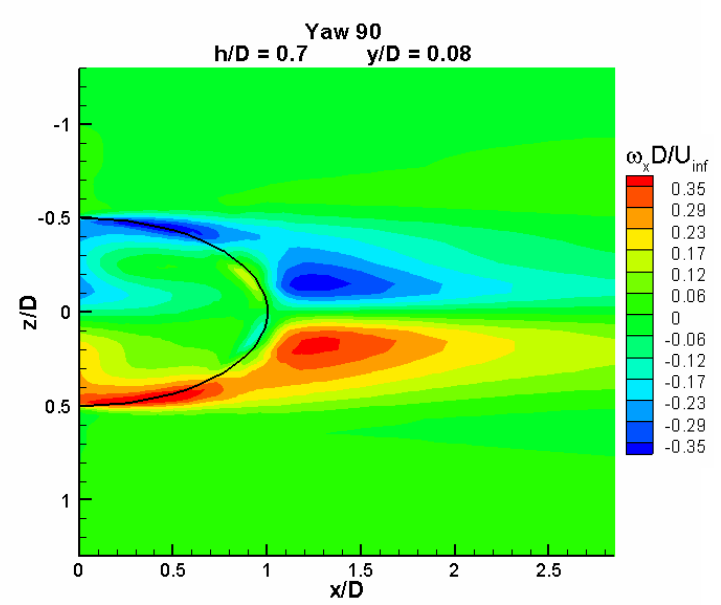

Figure 5-55 Streamwise vorticity contours at $\mathrm{y} / \mathrm{D}=0.08$ for $\mathrm{h} / \mathrm{D}=0.7$ at $90^{\circ}$ yaw angle

\subsubsection{Flow Dynamics}

As previously mentioned in section 5.3.5, the results of previous studies involving nominally two-dimensional rectangular cavities have shown that the flow inside the cavity is more stable for deeper cavities [26]. The results of the experimental study by Hering [58] and the present results showed a similar behaviour for elliptical cavities in the symmetric flow regime ( $0^{\circ}$ yaw angle). Based on the results of the POD analysis, this behaviour was attributed to oscillations of the vortex inside the cavity, which were found to have a larger amplitude for shallower cavities. In this section, the existence of a similar relationship for cavities in the nominally two-dimensional regime $\left(90^{\circ}\right.$ yaw angle) will be examined. 
Figure 5-56 compares distributions of $\mathrm{u}_{\mathrm{rms}}$ in the cavity mid-plane $(\mathrm{z} / \mathrm{D}=0)$, as an indicator of flow oscillations. The figure indicates an increasingly higher level of $\mathrm{u}_{\mathrm{rms}}$ in the cavity when $h / D$ decreases from 1.0 to 0.5 . Distributions of $u_{r m s}$ and $v_{r m s}$ on a vertical line located at $x / D=0, z / D=0$, show in Figure 5-57, confirm this behaviour quantitatively.

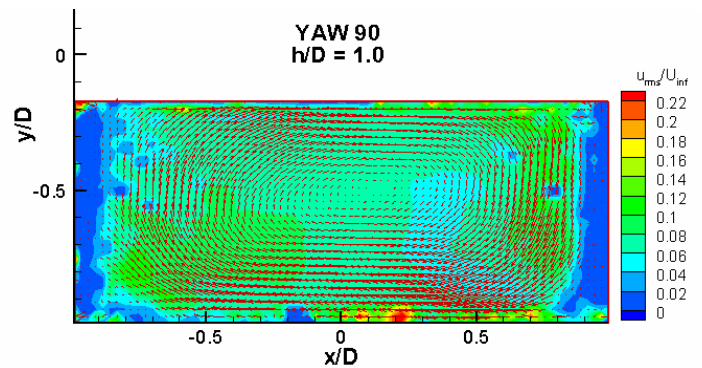

(a)

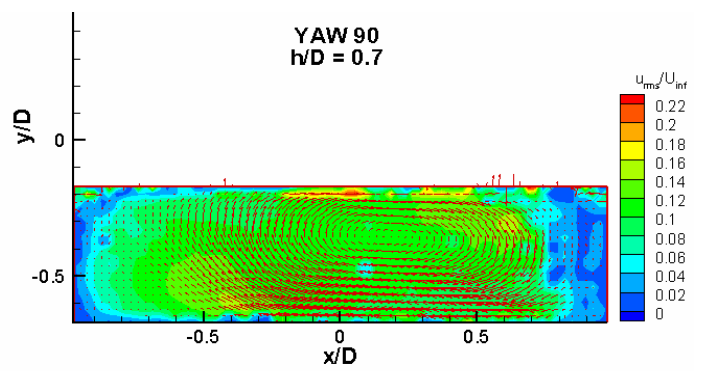

(b)

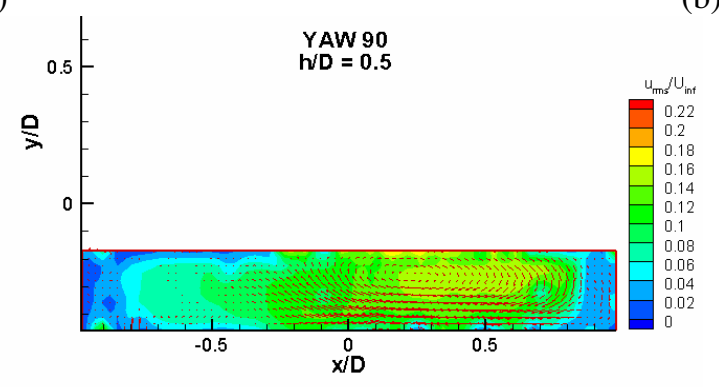

(c)

Figure 5-56 $u_{\mathrm{rms}}$ contours for $\mathrm{h} / \mathrm{D}=1.0(\mathrm{a}), 0.7(\mathrm{~b})$, and $0.5(\mathrm{c})$ at $90^{\circ}$ yaw angle at $\mathrm{z} / \mathrm{D}=0$

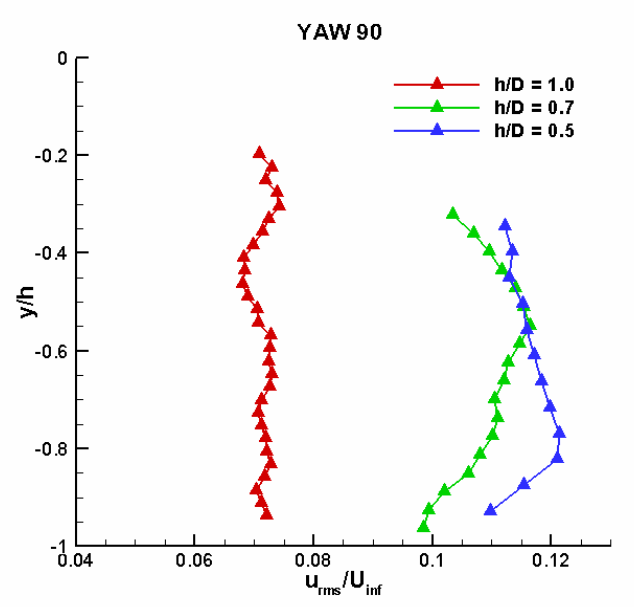

(a)

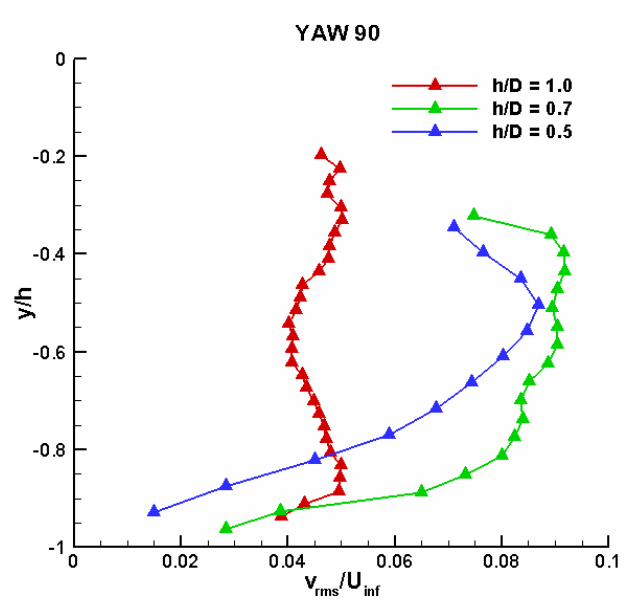

(b)

Figure 5-57 Comparison of $u_{\mathrm{rms}}(a)$ and $v_{\mathrm{rms}}$ (b) profiles on the vertical centreline 
A quantitative comparison of the fluctuating velocity components on the cavity vertical centreline $(\mathrm{x} / \mathrm{D}=0)$ with those of the cavity at $0^{\circ}$ yaw angle (Figure 5-26) indicates higher levels of $\mathrm{u}_{\mathrm{rms}}$ and $\mathrm{v}_{\mathrm{rms}}$ for the cavity with $90^{\circ}$ yaw angle. For example, the maximum value of $\mathrm{u}_{\mathrm{rms}} / \mathrm{U}_{\text {ref }}$ increases from 0.04 to 0.07 for $\mathrm{h} / \mathrm{D}=1.0$, and from 0.07 to 0.12 for $h / D=0.5$. The increased levels of $u_{r m s}$ for the cavity with $90^{\circ}$ yaw angle is due to the larger distance of the centreline from the edge of the upstream wall (which is twice as large as that of the cavity with $0^{\circ}$ yaw angle). As mentioned in section 2.4 , previous studies involving nominally two-dimensional rectangular cavities have shown that the level of velocity fluctuations in the separated shear layer and inside the cavity grows with increasing distance from the edge of the upstream wall [25] and [28]. The present results confirm this behaviour in the case of elliptical cavities.

Similar to the case of the cavity with $0^{\circ}$ yaw angle, POD analysis has been used in this section to investigate the dynamic behaviour of the flow inside the cavity, and its relationship with the fluctuating velocities inside the cavity. The POD analysis has been carried out based on the results of PIV measurements in the vertical plane located at z/D $=0$, for cavities with $\mathrm{h} / \mathrm{D}=0.5$ and 1.0 .

The first four POD modes of the fluctuating streamwise and vertical velocity components are shown in Figure 5-58 and Figure 5-59. A pair-wise relationship similar to that described for the cavity at $0^{\circ}$ yaw angle (section 5.3.5) can be observed between the POD modes, suggesting that Modes 1 and 2 point to the same dynamic behaviour in the flow, and are temporally related. The relative energies $\left(\lambda_{i} / \sum_{i=1}^{n} \lambda_{i}\right)$ of the first forty POD modes are shown in Figure 5-60 for the cavities with $\mathrm{h} / \mathrm{D}=1.0$ and 0.5 , and indicate that the first two POD modes contain $15.8 \%$ and $42.0 \%$ of the total energy of the velocity field, for $\mathrm{h} / \mathrm{D}=1.0$ and 0.5 , respectively. As mentioned previously in section 5.3.5, these ratios are smaller that the typical values reported by Murray et al. [25] and Savory et al. [35], because a major part of the shear layer, which contains the highest levels of velocity fluctuations, is not included in the results of the present PIV measurements due to the masking caused by the base plate. 


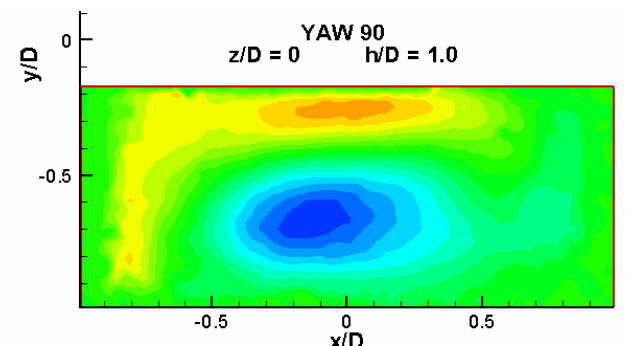

(a)

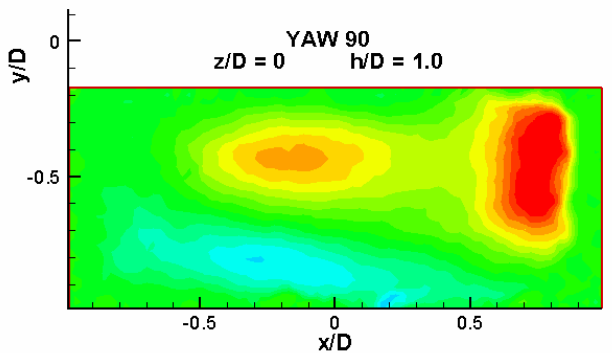

(c)

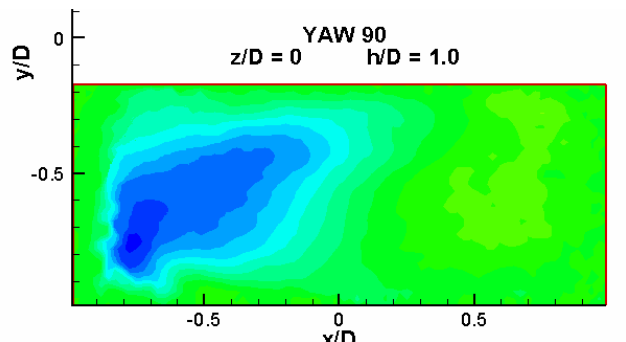

(b)

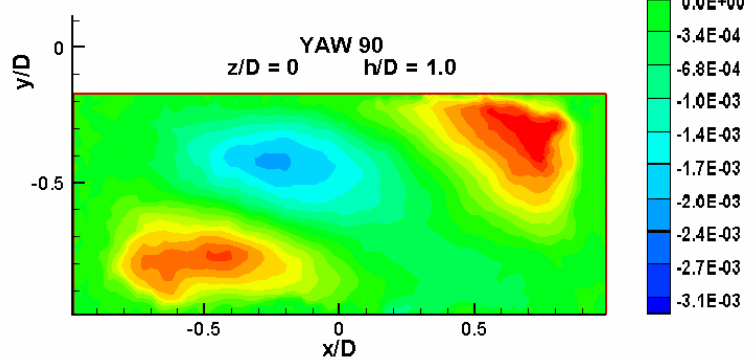

(d)

Figure 5-58 The first four POD modes of the fluctuating streamwise velocity component

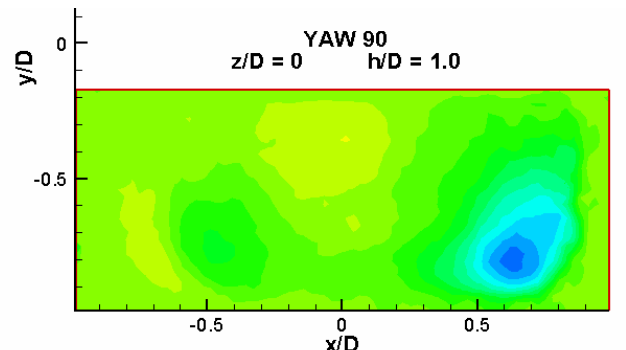

(a)

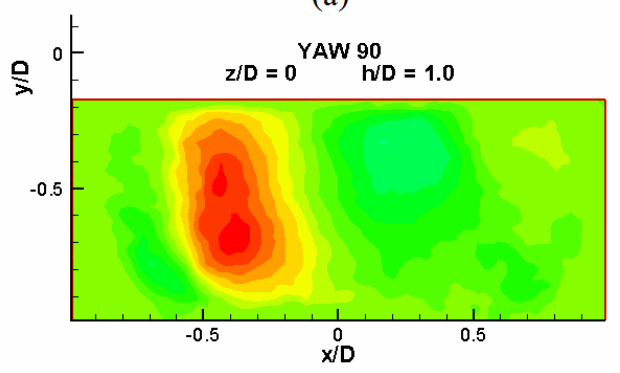

(c)

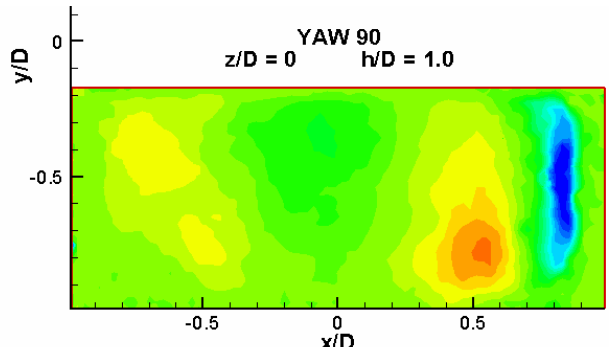

(b)

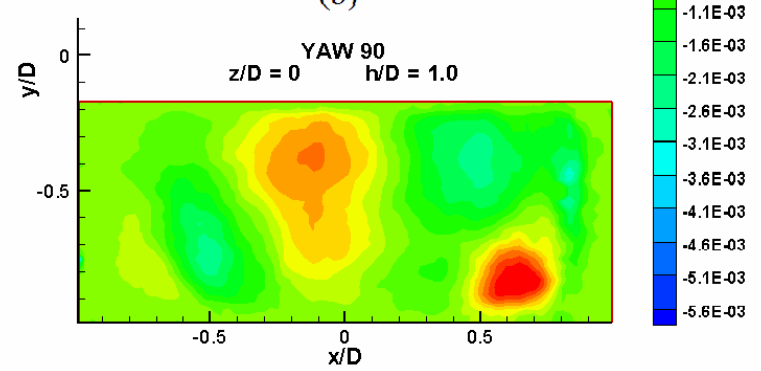

(d)

Figure 5-59 The first four POD modes of the fluctuating vertical velocity component

Plots of normalized time-varying coefficients $a_{1}(t) / \sqrt{2 \lambda_{1}}$ vs. $a_{2}(t) / \sqrt{2 \lambda_{2}}$, which are shown in Figure 5-61, confirm the temporal relationship between the two modes, and suggests a 90-degree phase difference, as previously described in the case of the cavity with $0^{\circ}$ yaw angle. This relationship has been utilized to determine the phase angle $\theta$ for 
each PIV snapshot using Equation 5-2, which is then used to calculate phase averaged velocity fields, in an approach similar to that implemented by Savory et al. [35].
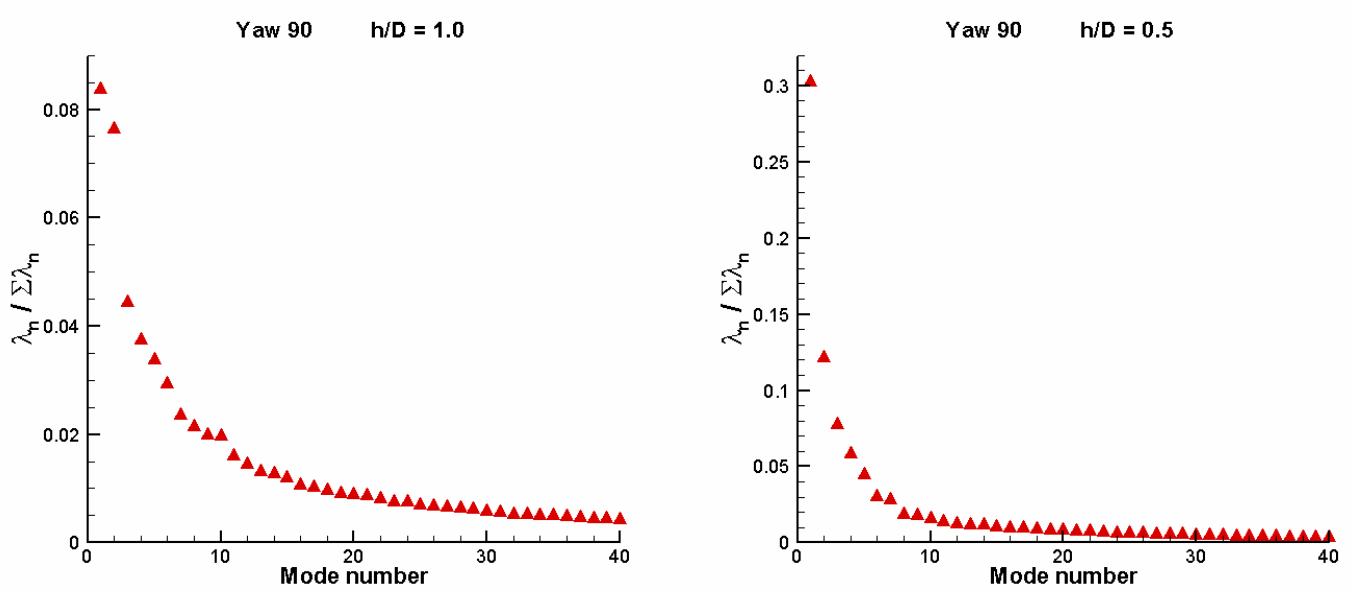

Figure 5-60 The first forty normalized eigenvalues for $h / D=$

1.0 (a) and 0.5 (b) at $90^{\circ}$ yaw angle at $\mathrm{z} / \mathrm{D}=0$

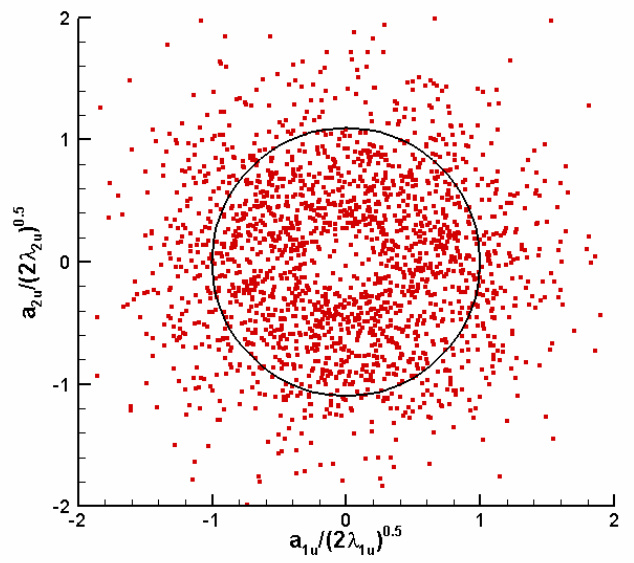

(a)

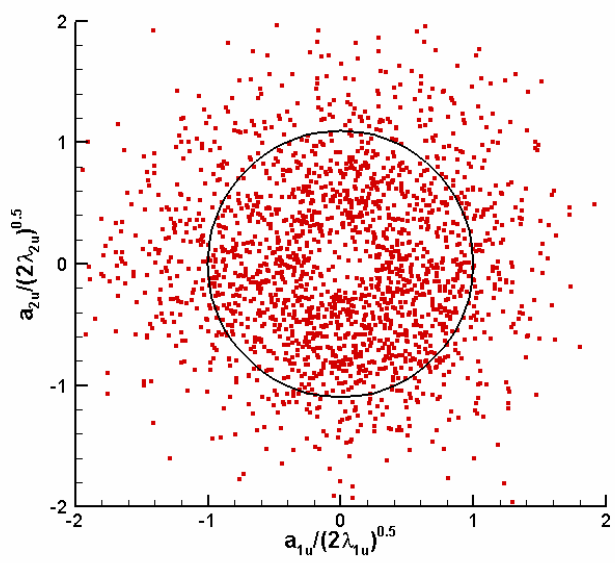

(b)

Figure 5-61 Normalized time-varying coefficients for the cavities with $h / D=1.0$ (a) and 0.5 (b)

Plots of streamtraces in the cavity based on the phase averaged velocity fields are shown in Figure 5-62 and Figure 5-63, for $\mathrm{h} / \mathrm{D}=1.0$ and 0.5 respectively. The plots show vortex oscillations inside the cavity for both $\mathrm{h} / \mathrm{D}$ ratios. Similar to the case of the cavity with $0^{\circ}$ yaw angle (section 5.3.5), the amplitude of the oscillations is found to be larger for the cavity with $h / D=0.5$. As a quantitative measure of the oscillations, variations in the 
position of the vortex core in the streamwise and vertical directions are 0.07D and $0.12 \mathrm{D}$ for the cavity with $h / D=1.0$, and $0.2 \mathrm{D}$ and $0.3 \mathrm{D}$ for the cavity with $\mathrm{h} / \mathrm{D}=0.5$. This observation agrees with the previously reported results indicating larger surface pressure and velocity fluctuations for cavities with smaller depths, and suggests that the higher fluctuating values are related to increased vortex oscillation.

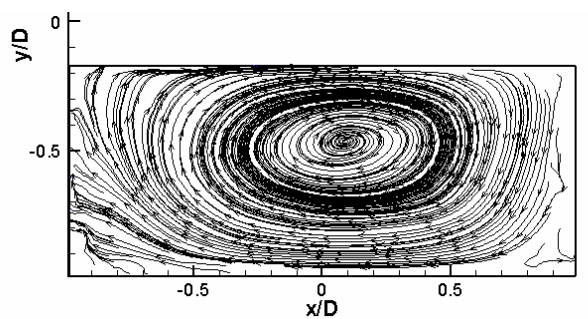

(a)

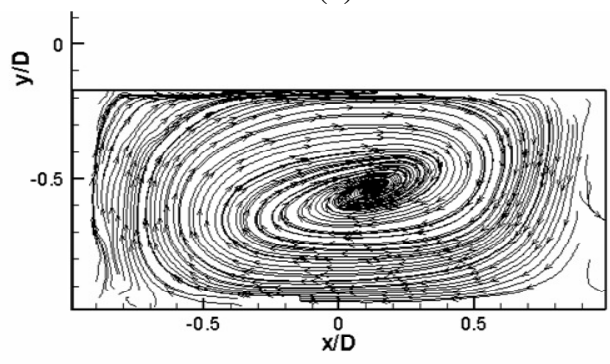

(c)

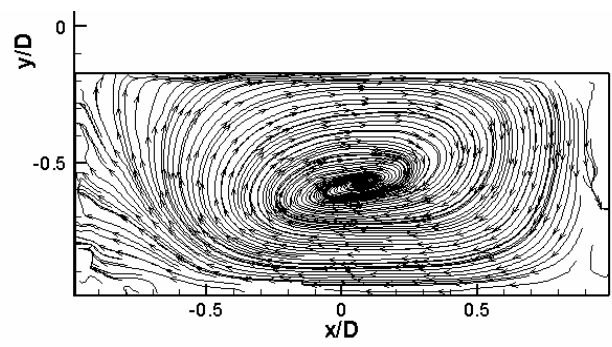

(b)

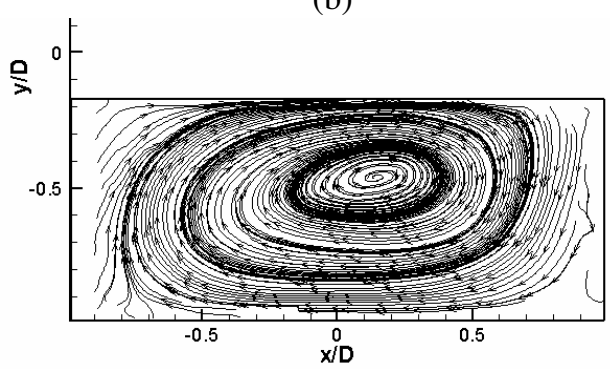

(d)

Figure 5-62 Phase averaged streamtraces in the vertical plane located at $\mathrm{z} / \mathrm{D}=\mathbf{0}$, for the cavity with $h / D=1.0$. The phase interval between consecutive plots is $90^{\circ}$

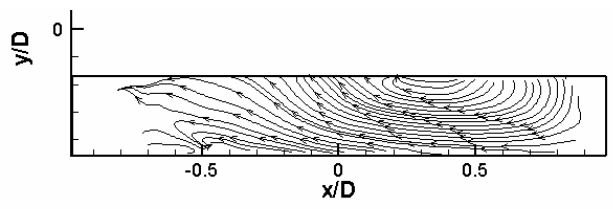

(a)

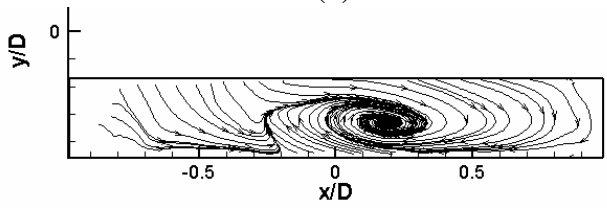

(c)

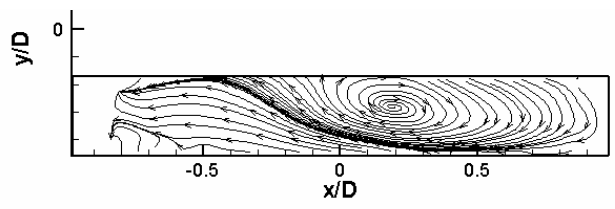

(b)

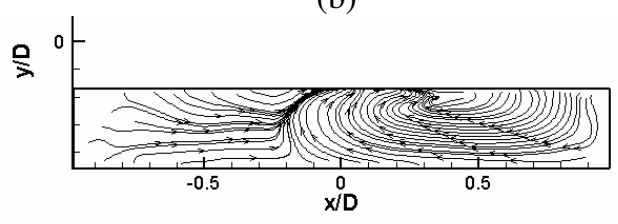

(d)

Figure 5-63 Phase averaged streamtraces in the vertical plane located at $\mathrm{z} / \mathrm{D}=0$, for the cavity with $h / D=0.5$. The phase interval between consecutive plots is $90^{\circ}$ 
A comparison of the amplitudes of vortex oscillations with those obtained for cavities at $0^{\circ}$ yaw angle (section 5.3.5) indicates that the amplitudes are larger for the cavity with $90^{\circ}$ yaw angle. This behaviour is consistent with the larger levels of velocity fluctuations in this case (Figure 5-57), and can be attributed to the streamwise length of the cavity opening, which is twice as large as that of the cavity at $0^{\circ}$ yaw angle, allowing for further growth of the fluctuations in the shear layer before impingement upon the downstream wall, as previously reported for nominally two-dimensional cavities by Murray et al. [25] and Manovski et al. [28].

As mentioned in section 5.3.5, the vertical oscillations of the vortex, and the fact that the present cavity is not in resonant conditions (section 2.5), suggests that a flapping instability in the shear layer similar to that reported by Haigermoser et al. [24] and Ukeiley and Murray [26] is responsible for the vortex oscillations.

\subsubsection{Summary of Findings for the Nominally Two-Dimensional Flow Regime}

The findings presented in this section regarding the nominally two-dimensional flow regime can be summarized as follows:

- For cavities with $\mathrm{h} / \mathrm{D}=1.0$ and 0.5 , behaviour of the shear layer is similar to that of deep nominally two-dimensional rectangular cavities in the "open" flow regime. The shear layer of the cavity with $\mathrm{h} / \mathrm{D}=0.1$ shows a behaviour similar to shallow rectangular cavities in the "closed" flow regime. For all cavity depths the shear layer grows almost linearly downstream of the separation point, with an average growth rate of $d \delta_{\omega} / d x=0.2$, which is within the range of the growth rate observed in other studies on cavity flows.

- The flow structure for the cavities with $h / D=1.0$ and 0.5 consists of a captive vortex similar to that of deep nominally two-dimensional cavities in the "open" regime. The position and orientation shows little variation across the span. However, due to the effects of the curved upstream wall, the primary vortex is 
formed further downstream compared to nominally two-dimensional rectangular cavities, and the secondary vortex is either very small, or non-existent (Figure 5-64 and Figure 5-65).

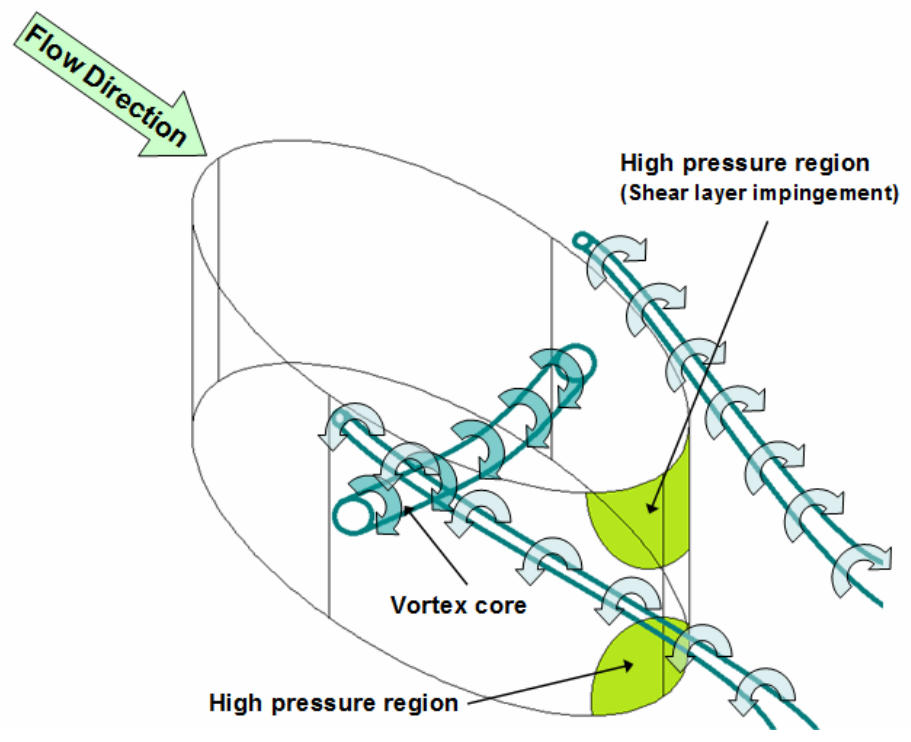

Figure 5-64 Schematic representation of the flow structure in an elliptical cavity in the nominally two dimensional-deep flow regime

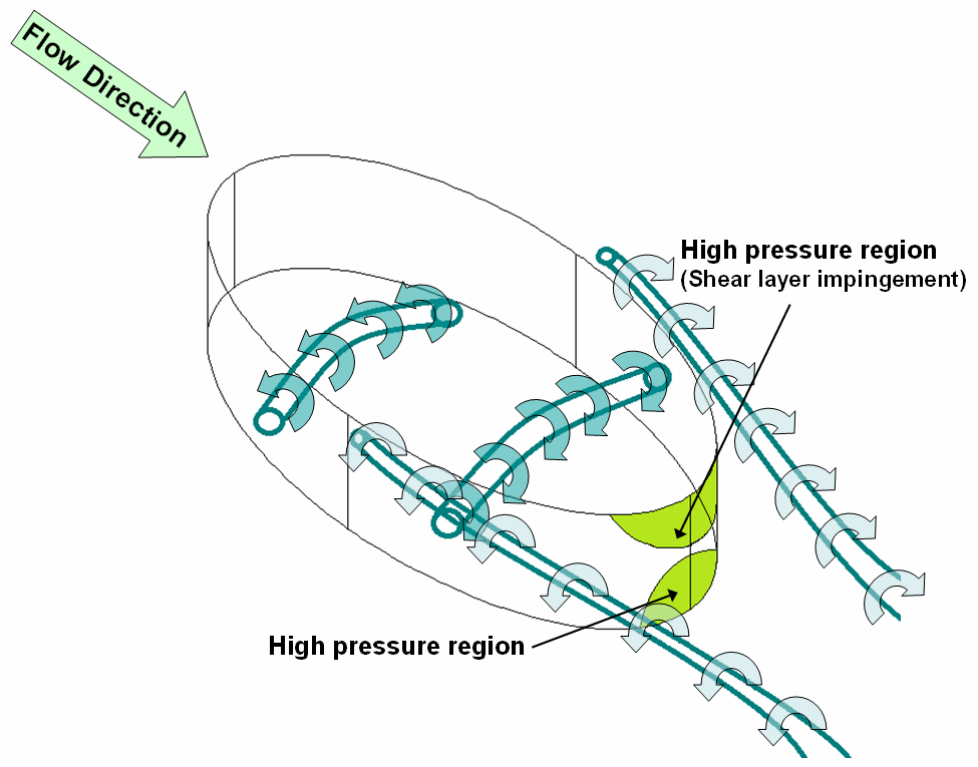

Figure 5-65 Schematic representation of the flow structure in an elliptical cavity in the nominally two dimensional-intermediate depth flow regime 
- For the cavity with $\mathrm{h} / \mathrm{D}=0.1$, the flow structure resembles that of shallow nominally two-dimensional cavities in the transitional flow regime, in which the shear layer is deflected into the cavity, but does not impinge onto the cavity base. The vortex core is parallel to the upstream wall of the cavity.

- The trends of variation of pressure on the centreline of the cavity base for all three $\mathrm{h} / \mathrm{D}$ ratios conforms to those reported by Plentovich [15] for nominally-twodimensional rectangular cavities with similar $\mathrm{h} / \mathrm{D}$ ratios.

- The trend of variation of drag with h/D ratio is found to be similar to that of the symmetric flow regime, in which higher drags are found for cavities with smaller $\mathrm{h} / \mathrm{D}$ ratios (shallower cavities). This behaviour has been attributed to the increased entrainment of the shear layer in the case of shallow and transitional cavities, which is confirmed by the flow structures presented in this section.

- The flow downstream of the cavity has been found to be affected by a pair of streamwise vortices originating from the cavity. The mechanism of formation and the sense of rotation of these vortices are different from the side edge vortices generated by a rectangular cavity and are related to separation of flow from the curved edge of the elliptical cavity (Figure 5-64 and Figure 5-65).

- Similar to cavities in the symmetric flow regime, higher levels of velocity fluctuations and time dependent displacement of the vortex are observed for the cavity with $\mathrm{h} / \mathrm{D}=0.5$, compared to that with $\mathrm{h} / \mathrm{D}=1.0$. Velocity fluctuations and vortex oscillations are found to be generally larger than those of cavities with $0^{\circ}$ yaw angle, due to the larger length of the cavity in the streamwise direction, which allows increased growth of the fluctuations in the shear layer. The vortex oscillations are possibly due to a flapping instability in the shear layer.

- Considering the differences found in the present study between the flow of elliptical cavities with $90^{\circ}$ yaw angle and that of nominally two-dimensional rectangular cavities due to the effects of curved sidewalls (such as the differences 
in secondary and side edge vortices), the term nominally two-dimensional should be used with caution for describing this flow regime.

\subsection{Asymmetric Flow Regimes}

Asymmetric flow regimes are associated with cavities with non-zero yaw angles. Unlike the cavities in symmetric and nominally two-dimensional regimes (discussed in sections 5.3 and 5.4), the flow in cavities in asymmetric flow regimes is not symmetric with respect to the mid-plane $(\mathrm{z} / \mathrm{D}=0)$.

As shown in Figure 5-1, the asymmetric flow regime is divided into two categories:

The weakly asymmetric regime, which includes yaw angles up to $30^{\circ}$, and the strongly asymmetric regime, which includes yaw angles equal to or larger than $45^{\circ}$.

In the weakly asymmetric regime, the non-zero yaw angle affects the flow inside and around the cavity. However, the extent of these effects is such that the flow structure in the cavity retains considerable similarity to that of the same cavity in the symmetric flow regime.

In the strongly asymmetric regime, however, the effects of yaw angle are much more pronounced, and the flow structure in the cavity is significantly different from that of the same cavity in the symmetric flow regime. Certain flow phenomena appear in the strongly asymmetric flow regime, which are unique to large, non-zero yaw angles.

The present study includes yaw angles of $15^{\circ}$ and $30^{\circ}$ as being representative of the weakly asymmetric regime, and $45^{\circ}$ and $60^{\circ}$ as representative of the strongly asymmetric regime. At each yaw angle, PIV measurements and numerical simulations have been carried out for $\mathrm{h} / \mathrm{D}$ ratios between 0.1 and 1.0. In this section, the results will be presented for $\mathrm{h} / \mathrm{D}=0.1,0.5$, and 1.0, which represent shallow, intermediate-depth, and deep flow regimes, respectively.

Various aspects of the flow, including shear layer behaviour, the three-dimensional flow structure, drag, flow downstream of the cavity, and the dynamic behaviour will be 
discussed in detail for the above mentioned combinations of yaw angle and $h / D$ ratio, and comparisons with previously published results will be included where comparable results are available.

\subsubsection{Behaviour of the Shear Layer}

As shown in sections 5.3 and 5.4, the behaviour of the separated shear layer, expressed in terms of the thickness of the shear layer, and the position of the shear layer centre, provides a valuable insight into the flow structure in the cavity.

Although several combinations of cavity $\mathrm{h} / \mathrm{D}$ ratio and yaw angle have been investigated in the present study, the effects of yaw angle on the shear layer parameters will be investigated for the cavity with $\mathrm{h} / \mathrm{D}=0.5$ as a representative case, to maintain the length of this section within reasonable limits.

Figure 5-66, which shows the position of the shear layer centre at three z/D locations, illustrates this effect for the cavity with $h / D=0.5$ at the yaw angle of $30^{\circ}$. The figure indicates that the height of the shear layer centre increases across the span, towards the downstream (positive-z) half of the cavity. This behaviour suggests that the vortex core forms at an increasingly higher position across the span, as z/D increases from -0.7 to 0.7 . As will be shown in the section 5.5.2, one of the features of the asymmetric flow structure in yawed cavities is the displacement of the cavity vortex in the vertical direction, which causes the vortex core to be closer to the upper edge of the cavity $(y / D=$ $0^{\circ}$ ) in the downstream (positive-z) half of the cavity.

Variations of the vorticity thickness along the upper edge of the cavity $(y / D=0)$ in the cavity mid-plane $(\mathrm{z} / \mathrm{D}=0)$, which have been determined based on the results of numerical simulations, are shown for yaw angles between $0^{\circ}$ and $90^{\circ}$ in Figure 5-67. The figure indicates an almost linear growth for the weakly asymmetric cases (yaw angles of $15^{\circ}$ and $30^{\circ}$ ). The growth rate in these cases is similar to those of the cavities in symmetric and nominally two-dimensional regimes $\left(d \delta_{\omega} / d x=0.2\right)$. The growth rate, however, becomes larger for the yaw angles associated with the strongly asymmetric regimes $\left(45^{\circ}\right.$ and $\left.60^{\circ}\right)$, and is non-linear for $60^{\circ}$ yaw angle. It will be shown in section 
5.5.2 that the vortex core exits the cavity volume for deep and intermediate-depth cavities at yaw angles of $45^{\circ}$ and $60^{\circ}$ as a result of extreme vertical displacement in the downstream (positive-z) half of the cavity. As a result, the behaviour of the shear layer at these two yaw angles is affected by the presence of the vortex at the upper edge of the cavity.

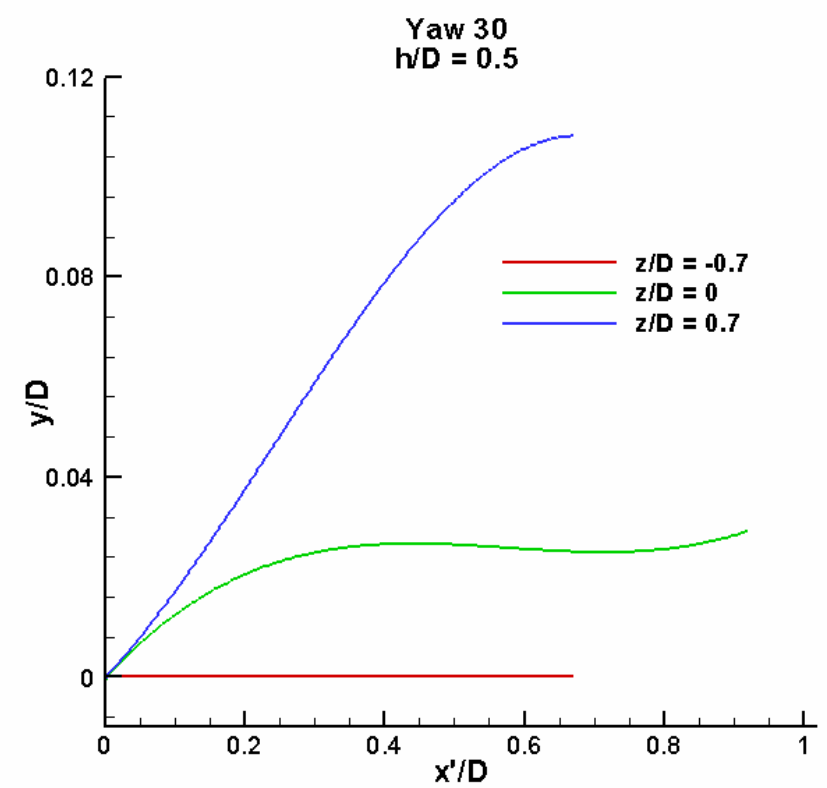

Figure 5-66 The location of shear layer centre at $30^{\circ}$ yaw angle

In summary, the asymmetric flow structure in yawed cavities is found to have significant effects on the shear layer parameters. It is, therefore, not possible to relate the behaviour of the shear layer for elliptical cavities in asymmetric flow regimes to those associated with nominally two-dimensional rectangular cavities in the "open" and "transitionalclosed" regimes. 


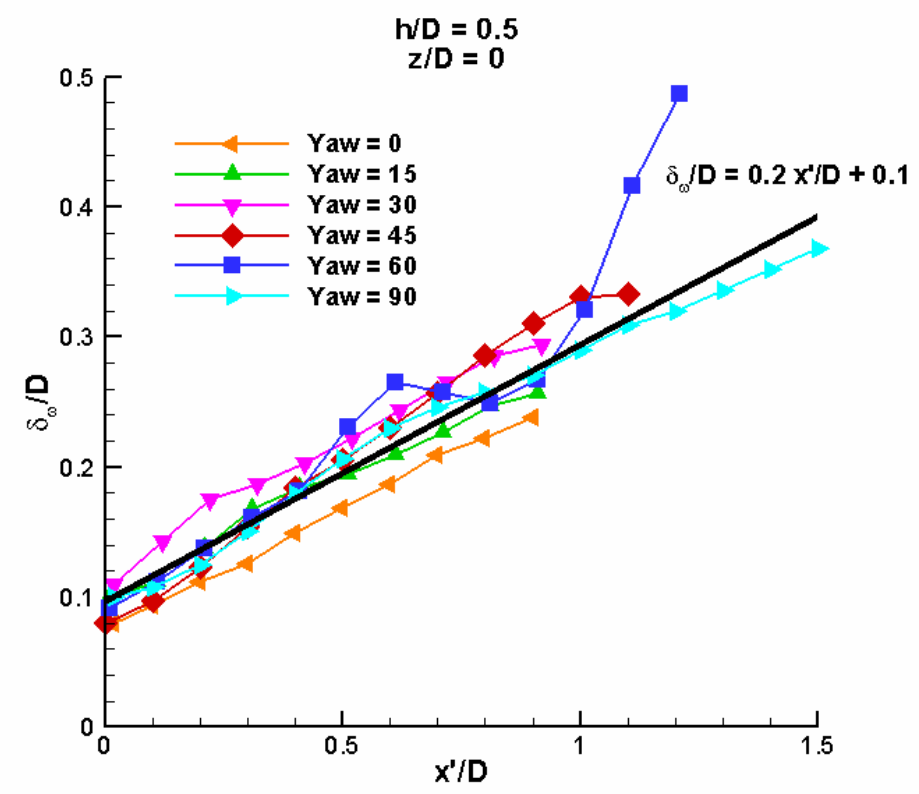

Figure 5-67 Shear layer thickness for $\mathrm{h} / \mathrm{D}=0.5$ at different yaw angles

\subsubsection{Flow Structure}

In the following paragraphs, a combination of the results of the PIV velocity field measurement, numerical simulations, and flow visualizations carried out in the present study, are used to identify and analyze the flow structure in elliptical cavities at various combinations of $\mathrm{h} / \mathrm{D}$ ratios and yaw angles associated with weak and strongly asymmetric flows. Comparisons with previously published results are included where available.

\subsubsection{Weakly asymmetric flows}

Figure 5-68 and Figure 5-69 show vortex core positions and surface pressure coefficient distributions for cavities with $\mathrm{h} / \mathrm{D}=1.0,0.5$, and 0.1 , at yaw angles of $15^{\circ}$ and $30^{\circ}$.

A comparison of the vortex core positions at the yaw angle of $15^{\circ}$ for all three $\mathrm{h} / \mathrm{D}$ ratios, with those of the respective cavities at $0^{\circ}$ yaw angle (section 5.3) indicates that the vortex for the yawed cavity retains the features of the vortex of the symmetric cavity, with slight changes due to yaw angle. 
For the cavity $\mathrm{h} / \mathrm{D}=1.0$ (Figure 5-68-(a)), the vortex core remains at a lower depth and close to the upstream edge of the cavity in the mid-plane (z/D $=0)$, and extends downwards and in the downstream direction as the distance from the centreline increases, due to the curvature of the upstream wall. For the cavity with $\mathrm{h} / \mathrm{D}=0.5$ (Figure 5-68-(c)), a W-shape vortex core, representative of a two cell structure continues to exist, and for the cavity with $\mathrm{h} / \mathrm{D}=0.1$ (Figure 5-68-(e)), the vortex core is formed near the upstream edge of the cavity and parallel to it.

An investigation of the pressure distributions at the yaw angle of $15^{\circ}$ (Figure 5-68) reveals the main difference between the flow structures for the cavities in the weakly asymmetric regime with those of the same cavities in the symmetric regime. Figure 5-68(b) indicates that for the deep cavity $(\mathrm{h} / \mathrm{D}=1.0)$, the low-pressure region associated with the vortex core is at a higher position (lower depth) on the positive-z half of the sidewall. A small high-pressure region is also formed at the base of the downstream wall of the cavity, in the positive-z half. The pressure distribution on the base of the cavity also shows a smaller low-pressure region in the positive-z half. These changes indicate that the vortex core is slanted in the vertical direction, such that the part of the vortex located in the positive-z half of the cavity is higher. A similar behaviour can be observed for the intermediate-depth cavity $(\mathrm{h} / \mathrm{D}=0.5)$ in Figure 5-68-(d). For the shallow cavity $(\mathrm{h} / \mathrm{D}=$ 0.1 , the low pressure region associated with the vortex core is slightly smaller in the positive-z half of the base, due to a slightly larger distance of the vortex core from the base (Figure 5-68-(f)).

The effects of yaw angle are more pronounced in the cavities with yaw angle of $30^{\circ}$. At this yaw angle, the vortex core in the cavities with $h / D=1.0$ and 0.5 lose their resemblance to their respective symmetric counterparts and are found to be almost parallel to the upstream wall of the cavity (Figure 5-69-(a) and (c)). The vortex core for the cavity with $\mathrm{h} / \mathrm{D}=0.1$, however, shows the least variation compared to the case with $15^{\circ}$ yaw angle, and tends to remain normal to the flow direction. As a result, the part of the vortex located in the positive-z half of the cavity is closer to the upstream wall (Figure 5-69-(e)). 


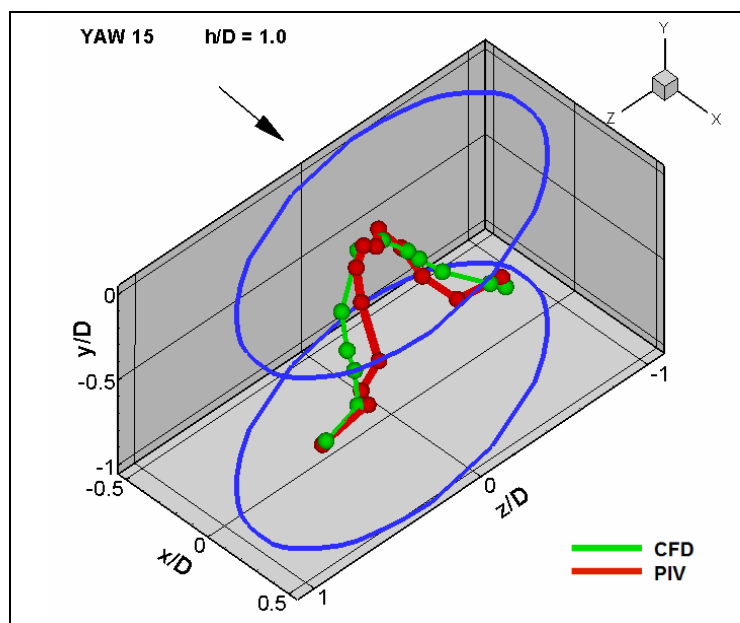

(a)

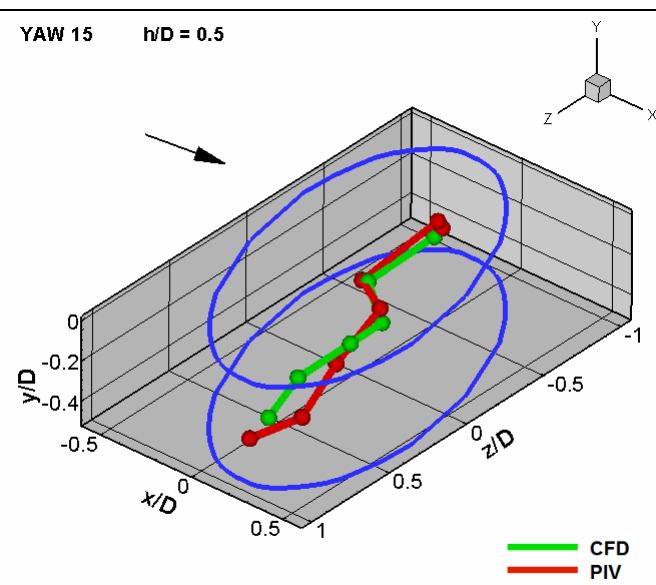

(c)

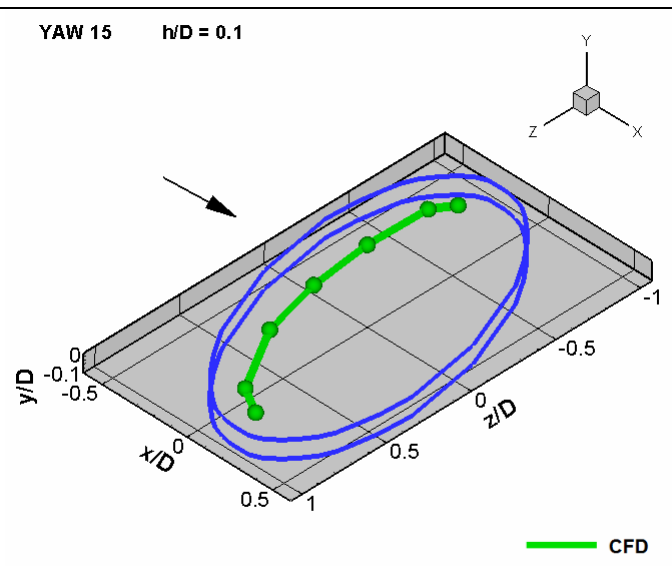

(e)
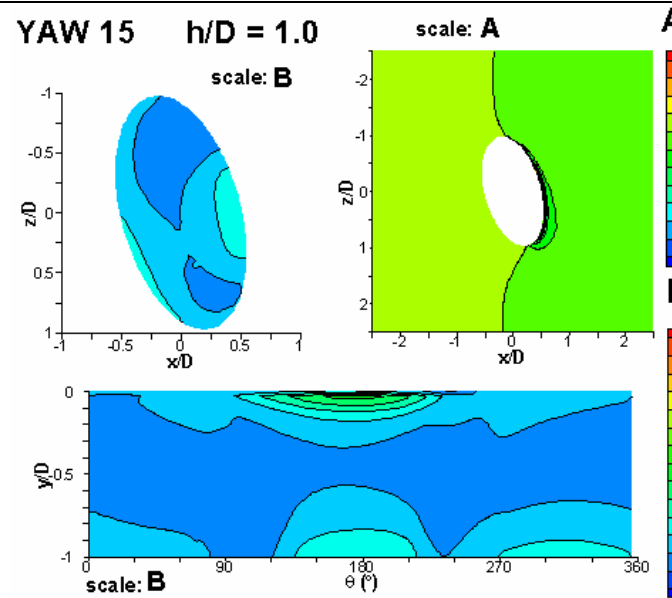

(b)
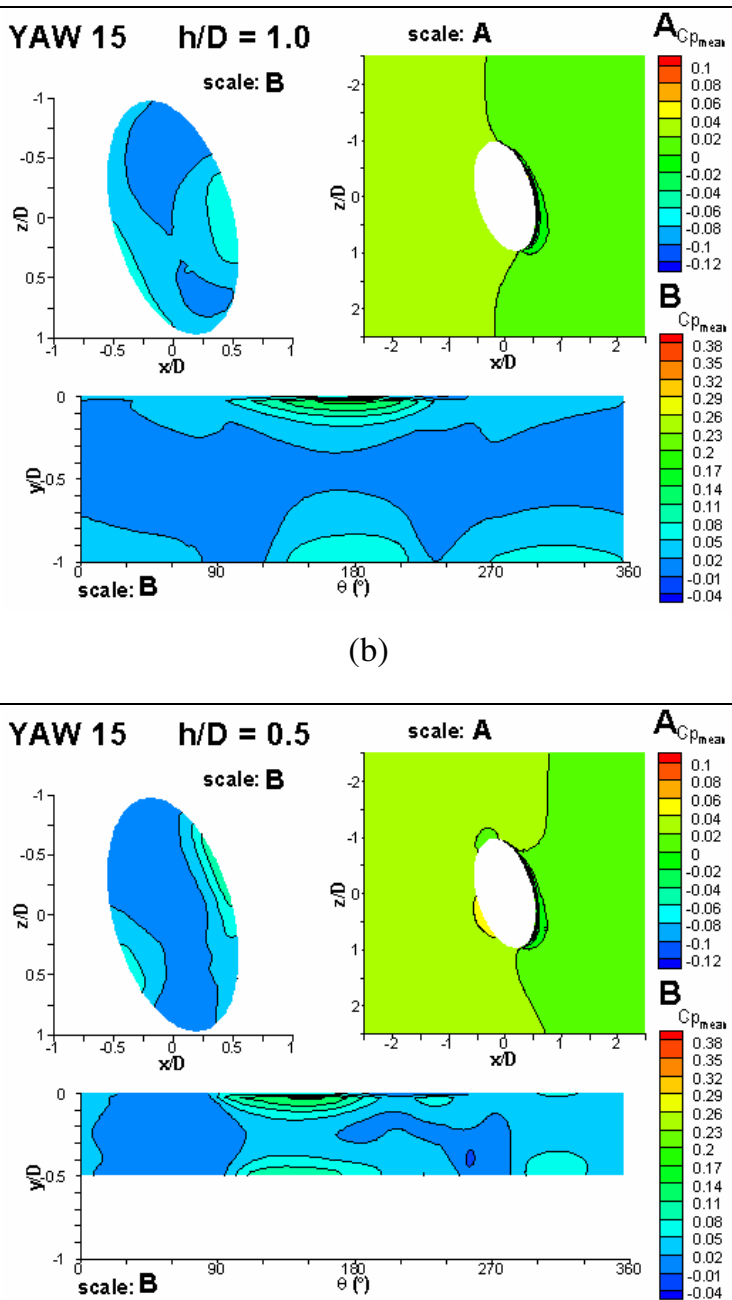

(d)
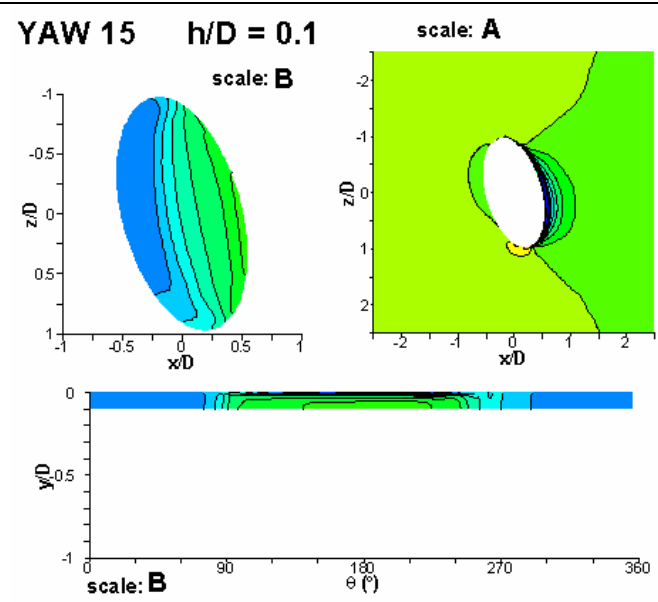

(f)

Figure 5-68 Vortex core positions and $C_{p}$ contours at yaw angle $=15^{\circ}$ for $h / D=1.0,0.5$ and 0.1 


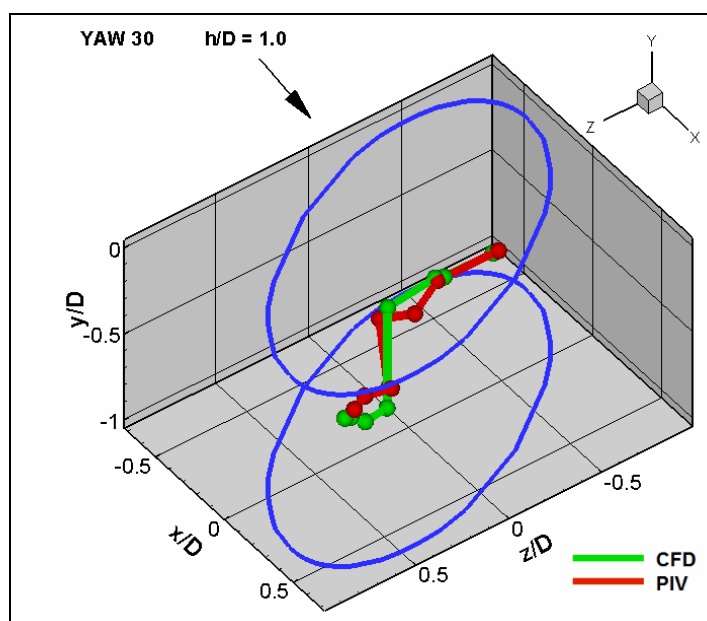

(a)

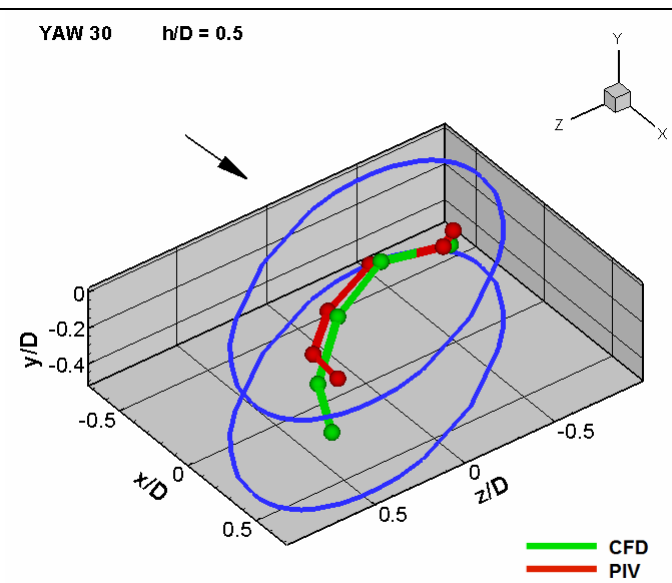

(c)

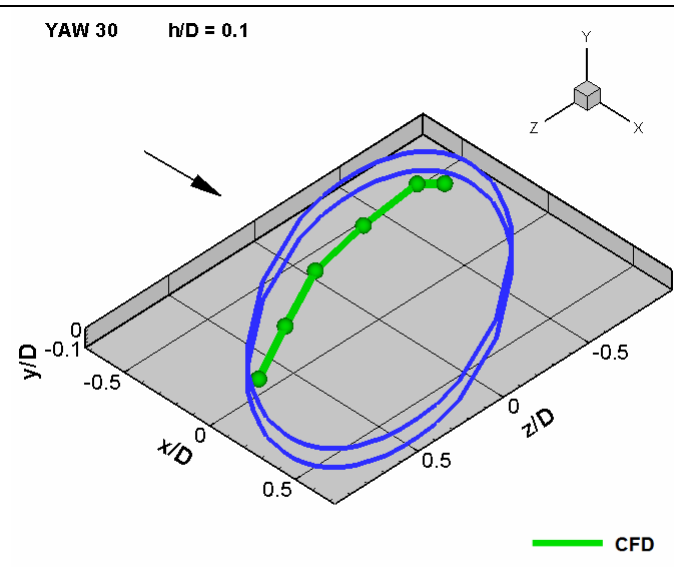

(e)
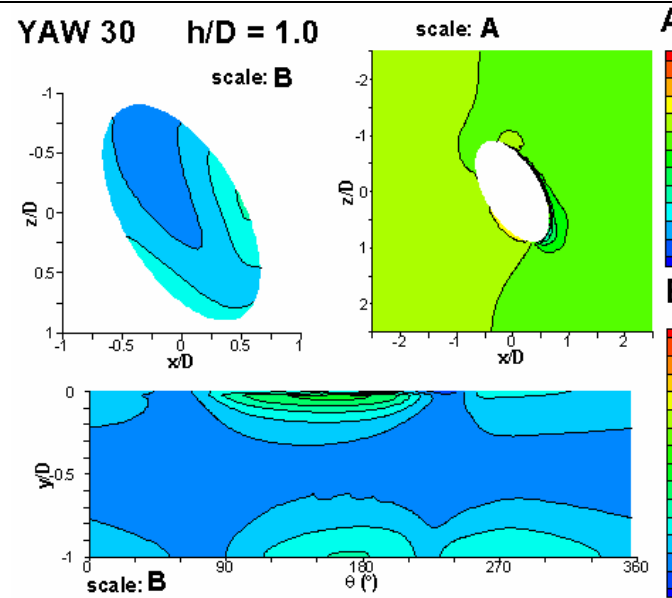

(b)
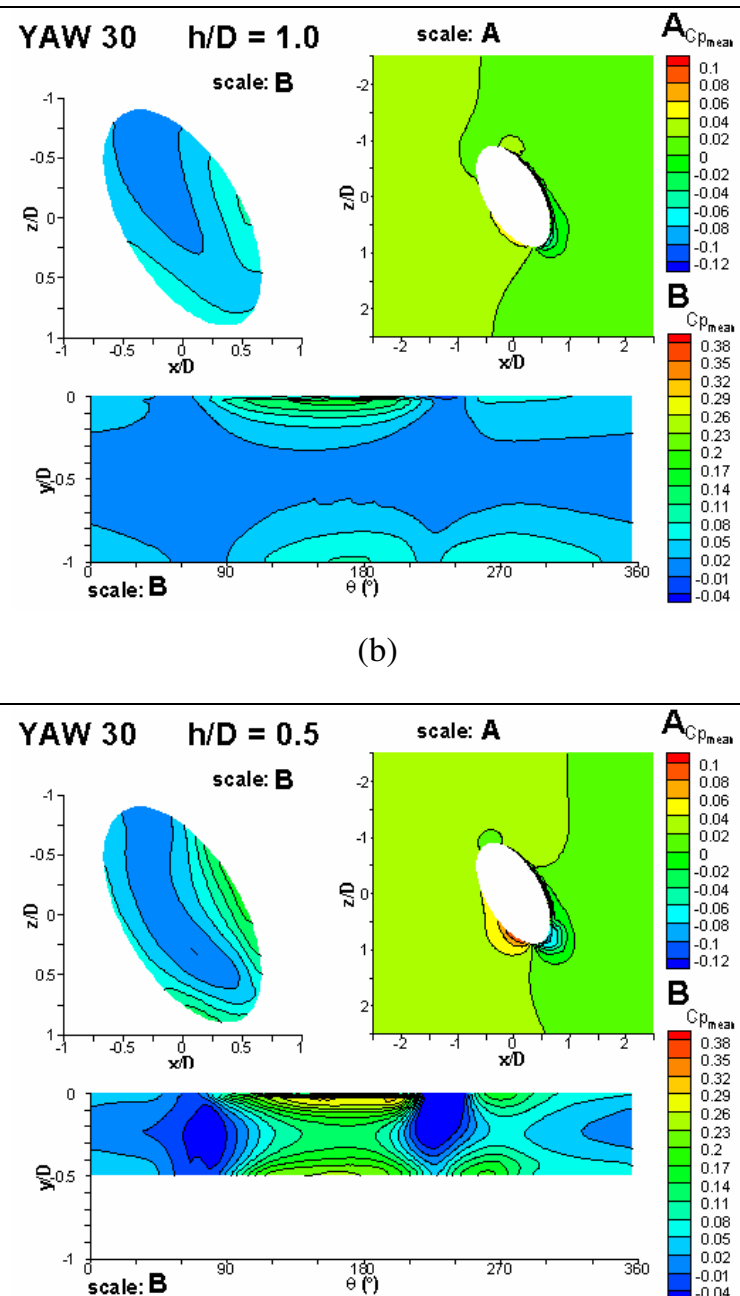

(d)
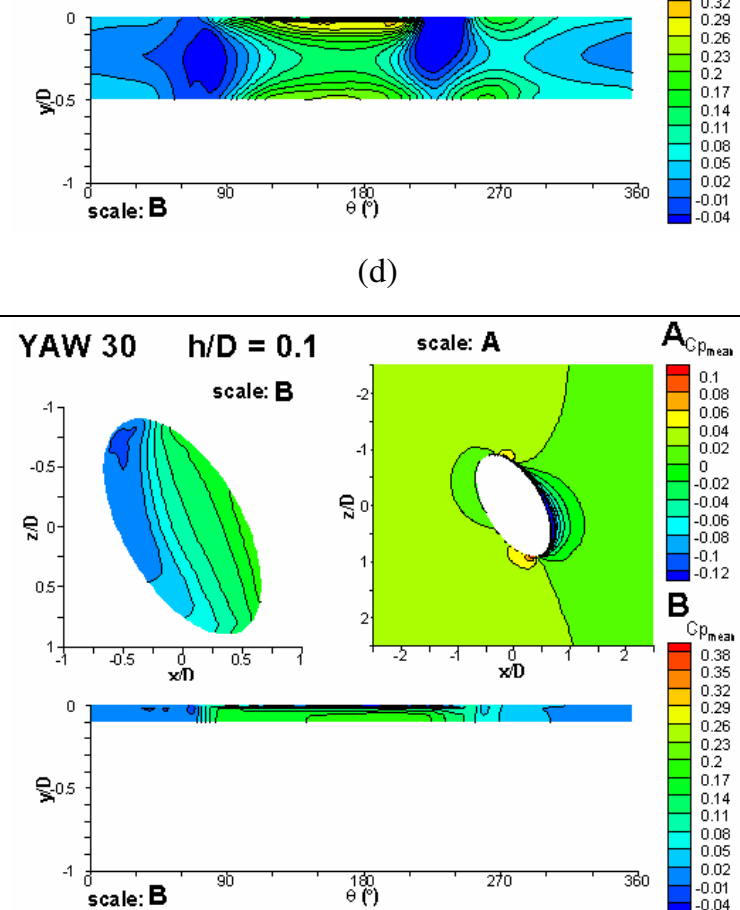

(f)

Figure 5-69 Vortex core positions and $C_{p}$ contours at yaw angle $=30^{\circ}$ for $h / D=1.0,0.5$ and 0.1 
An example of the effect of yaw angle on position of the vortex core is shown in Figure 5-70. The figure shows plots of streamtraces in vertical planes located at $\mathrm{z} / \mathrm{D}= \pm 0.7$, for the cavity with $\mathrm{h} / \mathrm{D}=0.5$, at the yaw angle of $30^{\circ}$. Comparison of the vortex core positions with those of the same cavity at $0^{\circ}$ yaw angle (highlighted by red dots in Figure 5-70) indicates that the vortex core is displaced vertically in the positive-z half of the cavity, and is formed farther away from the base. This behaviour will be explained in section 5.5.2.3.

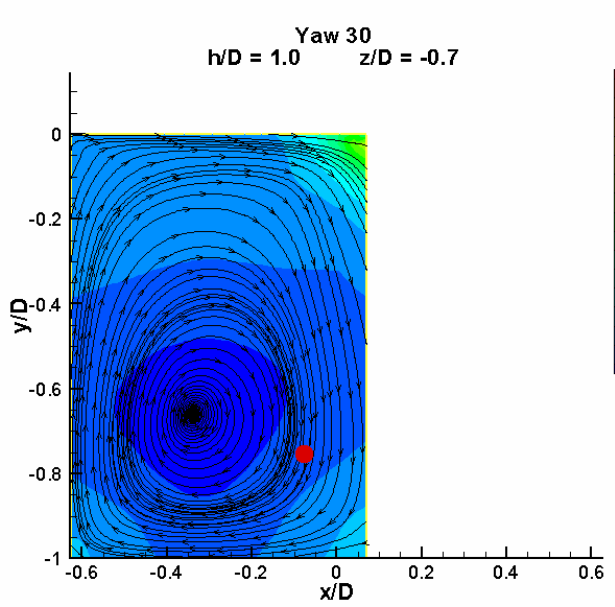

(a)
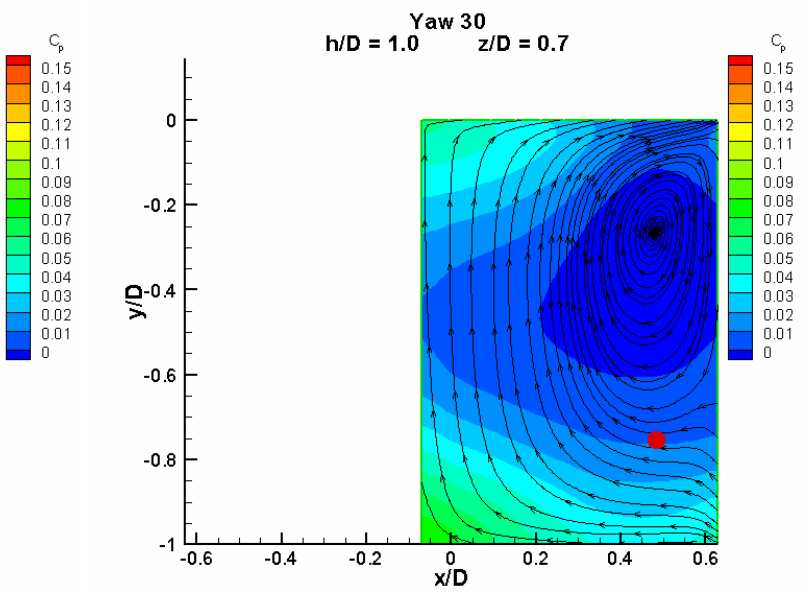

(b)

Figure 5-70 Streamlines and $C_{p}$ contours for cavity with $h / D=$

$$
1.0 \text { at } 30^{\circ} \text { yaw angle at } \mathrm{z} / \mathrm{D}=\mathbf{- 0 . 7}(\mathrm{a}) \text { and } \mathrm{z} / \mathrm{D}=\mathbf{0 . 7}(\mathrm{b})
$$

The surface pressure distributions, shown in Figure 5-69, also show the asymmetric flow structure and vertical displacement of the vortex core at the yaw angle of $30^{\circ}$ and indicate that this displacement is larger compared to the yaw angle of $15^{\circ}$ (Figure 5-68).

The surface pressure coefficient contours based on the experiments by Hering [58] and Czech [49] are shown in Figure 5-71 for comparison. Figure 5-71-(a) shows pressure coefficients for the elliptical cavity with $\mathrm{h} / \mathrm{D}=0.5$, at yaw angle of $30^{\circ}$, and indicate pressure distributions similar to those obtained based on the present numerical simulations (Figure 5-69-(b)). Considering the correspondence between the surface pressure distribution and the position of the vortex in the cavity (section 5.2.2), it can be concluded that the vortex structures in Hering's experiments are similar to those 
identified based on the present results. Figure 5-71-(b) shows pressure coefficients for a rectangular cavity with an aspect ratio of $2: 1$, with $\mathrm{h} / \mathrm{D}=0.5$, at yaw angle of $30^{\circ}$, and indicate behaviour similar to that of the elliptical cavity. The pressure distributions are almost similar to that of $0^{\circ}$ yaw angle (Figure 5-14), with the low-pressure region associated with the vortex core at a higher position on the positive-z sidewall. This effect is also confirmed by the pressure distribution on the cavity base. A similar effect is observed in the results of the study by Savory et al. [48] involving rectangular cavities with $2: 1$ aspect ratio. Their results indicate an asymmetric flow in the cavity with $h / D=$ 0.5 at $30^{\circ}$ yaw angle, in which the vortex remains parallel to the upstream wall, but its downstream end is at a higher position.

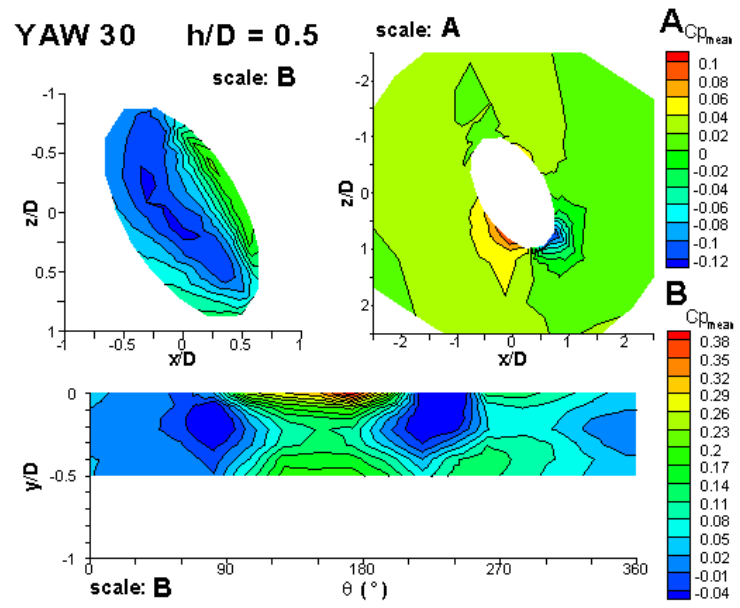

(a)

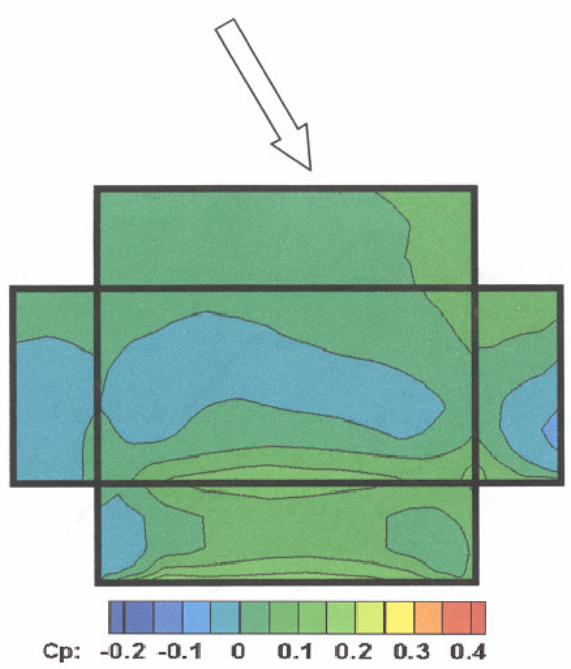

(b)

Figure 5-71 $C_{p}$ contours at yaw angle $=30^{\circ}$ for $h / D=0.5$ for a 2:1 elliptical cavity [58] and a 2:1 rectangular cavity (adapted from ref. [49])

\subsubsection{Strongly asymmetric flows}

Figure 5-72 and Figure 5-73 show vortex core positions and surface pressure coefficient distributions for cavities with $\mathrm{h} / \mathrm{D}=1.0,0.5$, and 0.1 , at yaw angles of $45^{\circ}$ and $60^{\circ}$.

Figure 5-72-(a) shows that for the cavity with $\mathrm{h} / \mathrm{D}=1.0$, the vertical displacement of the vortex core in the downstream (positive-z) half of the cavity has become more pronounced at the yaw angle of $45^{\circ}$. The downstream part of the vortex impinges onto 
the upper edge of the downstream wall $(y / D=0)$ at an angular position of $230^{\circ}$ approximately, and then exits the cavity volume to form a trailing vortex. Therefore, unlike the cavities with $\mathrm{h} / \mathrm{D}=1.0$ in symmetric, weakly asymmetric, and nominally-twodimensional regimes, the vortex can no longer be considered a captive one, typically associated with deep cavities.

A similar behaviour can be observed for the cavity with h/D ratio of 0.5 (Figure 5-72(c)). The vortex core is found to be parallel to the upstream wall of the cavity, similar to the case of the cavity with $\mathrm{h} / \mathrm{D}=0.5$. However, the vertical displacement of the vortex core in the downstream (positive-z) half of the cavity is more pronounced, so that the vortex core impinges onto the upper edge of the downstream wall at an angular position of $230^{\circ}$, approximately, and then exits the cavity volume to form a trailing vortex. A detailed description of the effect of the trailing vortex on the flow downstream of the cavity can be found in section 5.5.4.

The surface pressure coefficient distributions fort the cavities with $\mathrm{h} / \mathrm{D}=1.0$ and 0.5 (Figure 5-72-(b) and (d)) at the yaw angle of $45^{\circ}$ are consistent with the above-mentioned behaviour of the vortex cores. For both cases, a low-pressure region associated with the downstream part of the vortex can be observed at an angular position of $230^{\circ}$, at the upper edge of the cavity $(y / D=0)$. The high-pressure region associated with the impingement of the shear layer is no longer symmetric and is shifted in the upstream direction. Also, a high-pressure region can be observed at the base of the downstream wall, immediately underneath the location vortex core on the wall. The role of these features in the flow structure in the cavity will be described in more detail, later in this section.

At the yaw angle of $60^{\circ}$, a transition occurs in the flow structure of cavity with $\mathrm{h} / \mathrm{D}=1.0$. (Figure 5-73-(a)). Unlike the case with smaller yaw angles, in which the vortex core extends across the span of the cavity along the major axis, at a yaw angle of $60^{\circ}$ the vortex core is found to extend along the minor axis of the cavity, in a manner similar to the cavity with $90^{\circ}$ yaw angle (Figure 5-39). The vortex is bounded by the sidewalls at the angular positions of $150^{\circ}$ and $300^{\circ}$. Similar to the case of the cavity at $45^{\circ}$ yaw angle, 
the downstream part of the vortex core is displaced in the vertical direction, so that it impinges onto the upper edge of the downstream wall, and then exits the cavity to form a trailing vortex.

For the cavity with $\mathrm{h} / \mathrm{D}=0.5$, this transition does not occur at the yaw angle of $60^{\circ}$, and the vortex continues to extend along the major axis (Figure 5-73-(c)). Similar to the case of the cavity at $45^{\circ}$ yaw angle, the downstream part of the vortex core is displaced in the vertical direction, so that it impinges onto the upper edge of the downstream wall and then exits the cavity to form a trailing vortex.

The surface pressure coefficient distributions fort the cavities with $\mathrm{h} / \mathrm{D}=1.0$ and 0.5 at the yaw angle of $60^{\circ}$ (Figure 5-73-(b) and (d)) are consistent with the above-mentioned behaviour of the vortex cores. Figure 5-73-(b) shows two low pressure regions associated with the two ends of the vortex, at angular positions of $150^{\circ}$ and $300^{\circ}$. The low pressure region on the downstream wall is closer to the upper edge of the cavity $(y / D=0)$, indicating that the vortex is displaced in the vertical direction in the downstream (positive-z) half of the cavity. For the cavity with $\mathrm{h} / \mathrm{D}=0.5$ (Figure 5-73-(d)), a lowpressure region associated with the downstream part of the vortex can be observed at an angular position of $230^{\circ}$, at the upper edge of the cavity $(y / D=0)$.

Figure 5-74 shows an instantaneous visualization of the flow in the cavity with $\mathrm{h} / \mathrm{D}=1.0$ at the yaw angle of $45^{\circ}$, which verifies the flow structure described in the previous paragraphs. In this figure, a single bubble can be observed to be circulating near the upstream wall of the cavity, due to the upstream part of the vortex, while a few bubbles can be observed to be leaving the cavity volume in a helix-shaped path, following the downstream part of the cavity vortex. Due to the low contrast of the original image the circulation paths of the bubbles are highlighted. 


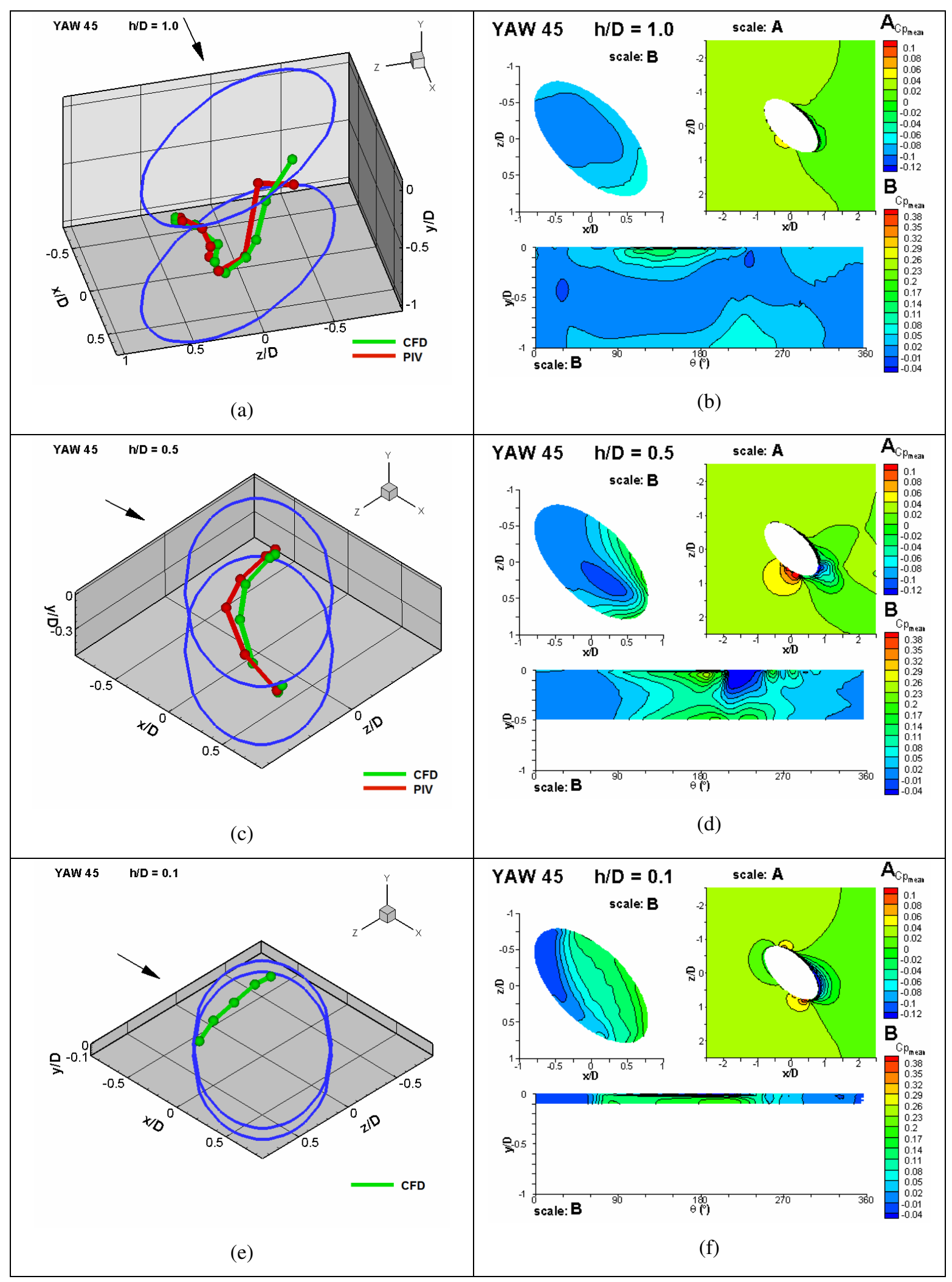

Figure 5-72 Vortex core positions and $C_{p}$ contours at yaw angle $=45^{\circ}$ for $h / D=1.0,0.5$ and 0.1 


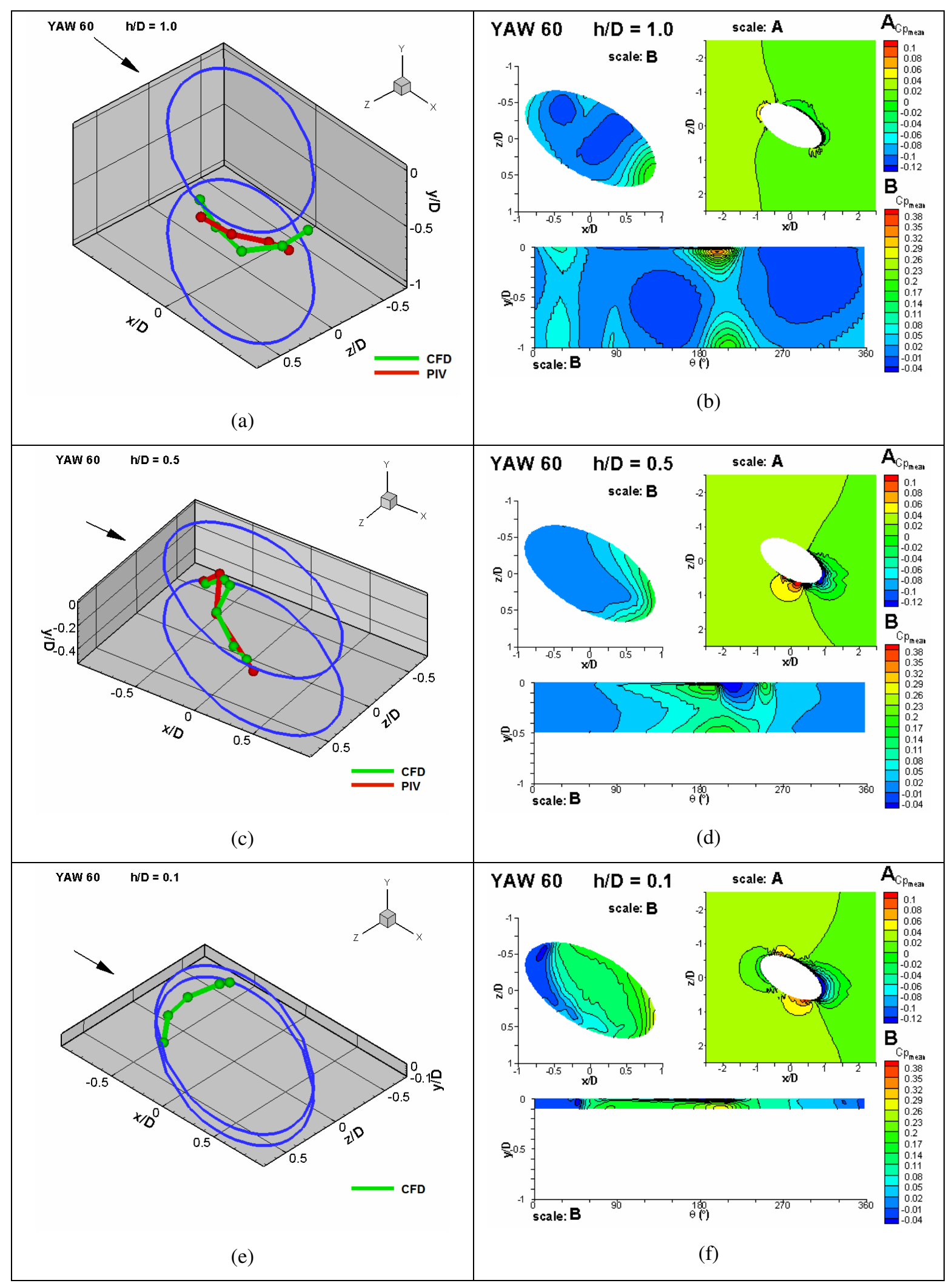

Figure 5-73 Vortex core positions and $C_{p}$ contours at yaw angle $=30^{\circ}$ for $h / D=1.0,0.5$ and 0.1 

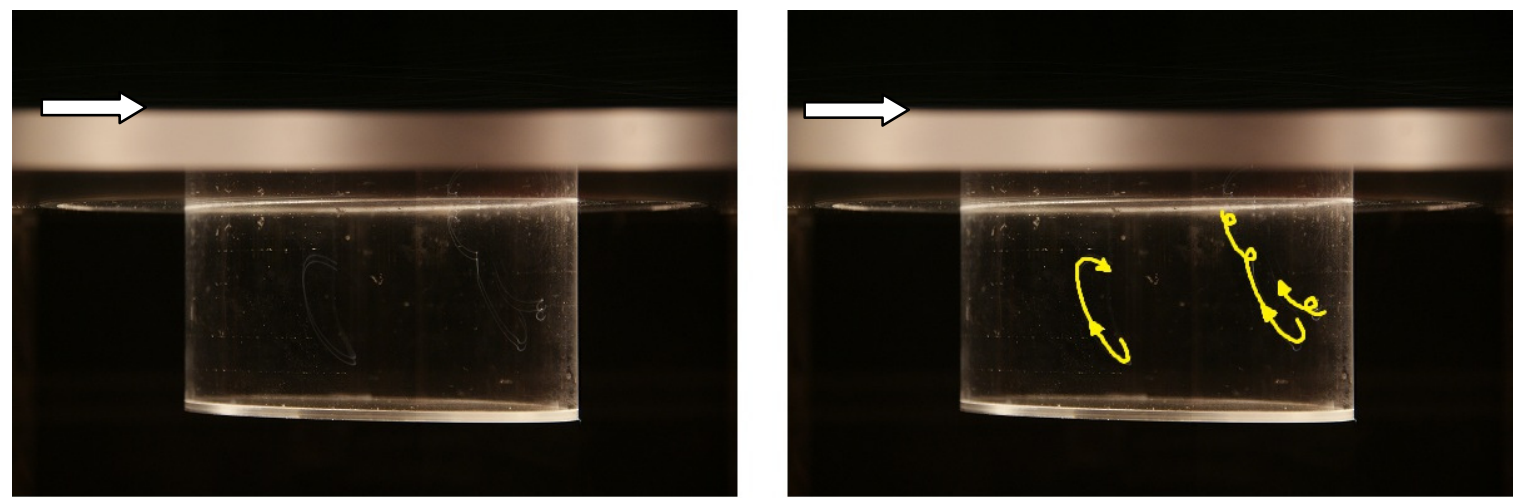

Figure 5-74 Instantaneous flow visualization image obtained using the Helium bubble technique, for the cavity with $h / D=1.0$ at yaw angle $=45^{\circ}$

The flow in the cavity with $h / D=0.1$ is found to have the least variation in the strongly asymmetric flow regime. Figure 5-72-(e) and Figure 5-73-(e) indicate that for both yaw angles of $45^{\circ}$ and $60^{\circ}$, the general orientation of the vortex core remains normal to the freestream. The vortex is close to the upstream wall of the cavity, with little displacement in the vertical direction in its downstream part. The pressure coefficient distributions shown in Figure 5-72-(f) and Figure 5-73-(f) indicate a slightly smaller low pressure region in the downstream (positive-z) half of the base, which may be due to the larger distance of the vortex core from the base. This effect is more pronounced at the yaw angle of $60^{\circ}$.

The surface pressure coefficient contours based on the experiments by Hering [58] and Savory et al. [5] are shown in Figure 5-75 for comparison. Figure 5-75-(a) shows pressure coefficients for the elliptical cavity with $\mathrm{h} / \mathrm{D}=0.5$, at yaw angles of $45^{\circ}$, and indicate pressure distributions similar to those obtained based on the present numerical simulations. The results of the study by Savory et al. [5] involving an elliptical cavity with the same geometric proportions, also indicate pressure distributions which are qualitatively similar to those of the present study (Figure 5-75-(b)), but are quantitatively different due to different upstream boundary layer parameters. 


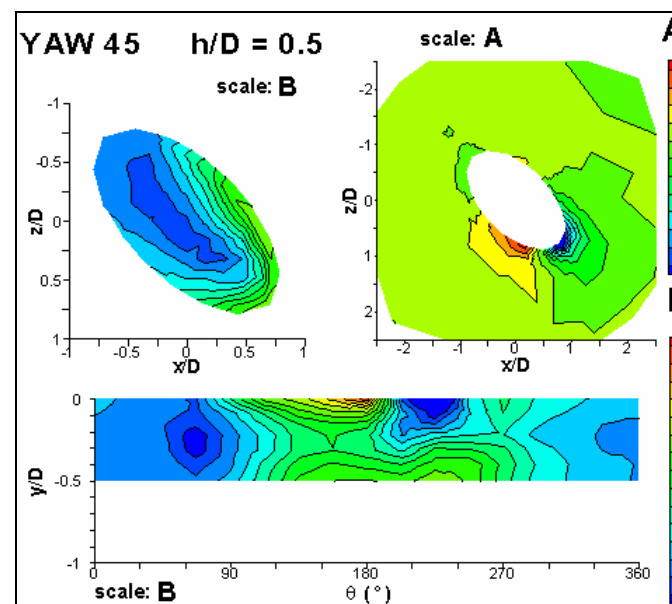

(a)

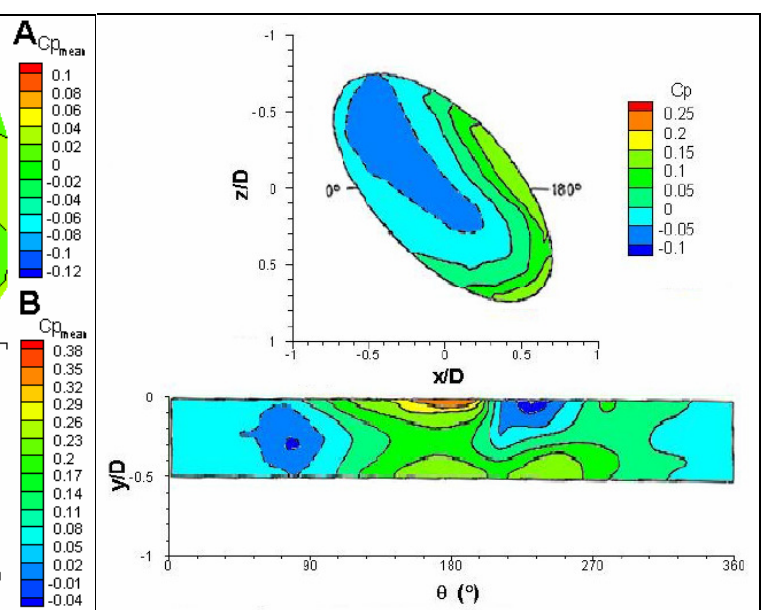

(b)

Figure 5-75 $C_{p}$ contours at yaw angle $=45^{\circ}$ for $h / D=0.5$, Hering

[58] (a), Savory and Toy (adapted from [5]) (b)

Figure 5-76 shows pressure coefficients for a rectangular cavity with an aspect ratio of $2: 1$, with $\mathrm{h} / \mathrm{D}=0.5$, at yaw angles of $60^{\circ}$. This figure shows lower pressures on the upstream half of the cavity base compared to the downstream half, and a low-pressure region close to the upper edge of the cavity, on the downstream wall. These pressure distributions indicate that the vortex inside the cavity is displaced in the vertical direction, with the upstream part closer to the cavity base, and the downstream part closer to the upper edge of the cavity.

The results of the study by Savory et al. [48] involving rectangular cavities with 2:1 aspect ratio show a behaviour similar to that of Czech's results at the yaw angles of $45^{\circ}$ [49]. According to their results, for the cavity with $h / D=0.5$ the vortex remains parallel to the upstream wall, but its downstream end is found to be at a higher position compared to its upstream end. For the yaw angle of $60^{\circ}$, their results indicate a complicated pressure distribution, from which it is not possible to determine whether the vortex core is aligned with the major or minor axis of the cavity. This behaviour suggests the possibility of a transition in the orientation of the vortex, similar to that observed in the present results for the cavity with $\mathrm{h} / \mathrm{D}=1.0$ (Figure 5-73-(a)).

The similar features of the flow structure in rectangular and elliptical cavities in the strongly asymmetric flow regime, including the vertical displacement of the vortex in the 
downstream part of the cavity, and formation of a trailing vortex, suggests that a similar mechanism is responsible for these features in both cases. This mechanism will be investigated in more detail in section 5.5.2.3.

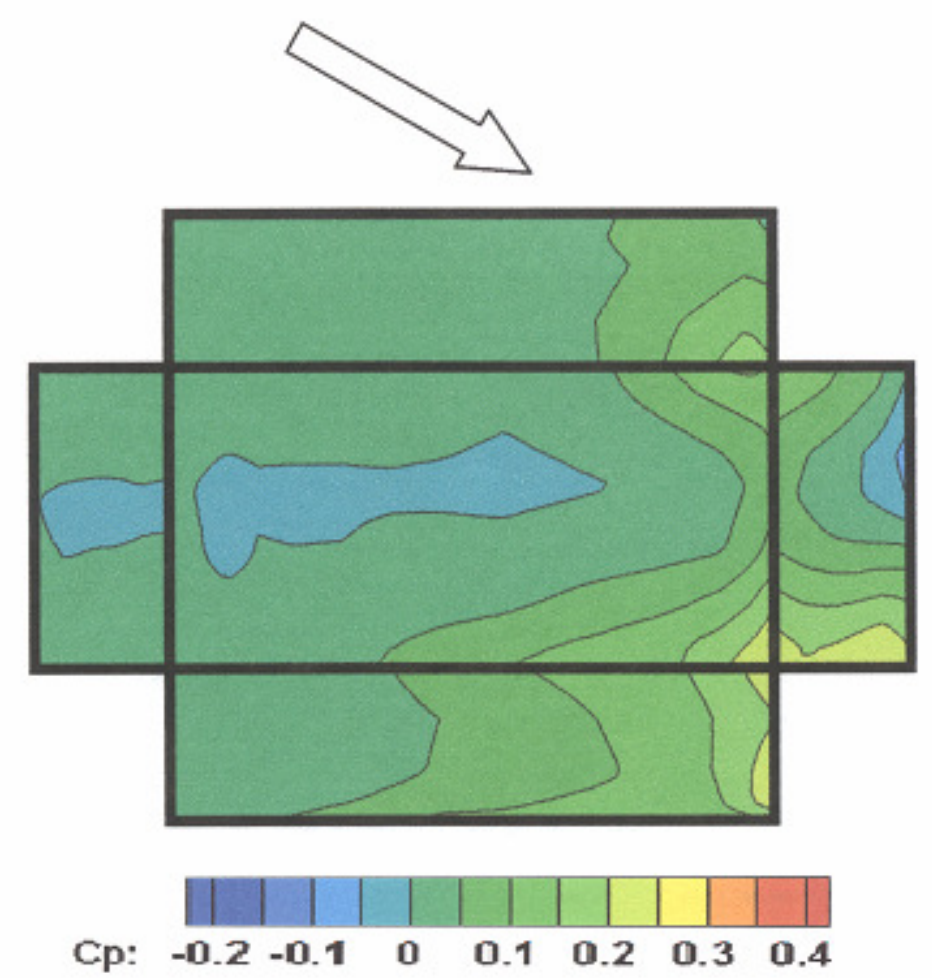

Figure 5-76 $C_{p}$ contours at yaw angle $=60^{\circ}$ for $h / D=0.5$ for a 2:1 rectangular cavity (adapted from ref. [49])

\subsubsection{Details of the flow in the strongly asymmetric regime}

As indicated by the results presented in the previous paragraphs and the literature cited for comparison, one of the prominent features of the flow inside deep and intermediatedepth cavities with elliptical and rectangular planforms, in the asymmetric flow regime, is the vertical displacement of the vortex in the downstream (positive-z) half of the cavity, which causes the vortex core to reach the upper edge of the cavity at the downstream wall. In order to explain this behaviour, a more in-depth investigation of the flow inside a cavity at high yaw angle will be presented in the following paragraphs. The cavity with $\mathrm{h} / \mathrm{D}=1.0$ at the yaw angle of $45^{\circ}$ has been chosen as a representative case, as it demonstrates the above-mentioned behaviour clearly. 
Figure 5-77-(a) and (b) show contours of pressure coefficient in two vertical planes inside the cavity, located at $\mathrm{z} / \mathrm{D}=-0.53$ and $\mathrm{z} / \mathrm{D}=0.53$, respectively. The first figure shows a high-pressure region near the upper edge of the cavity, which is associated with impingement of the separated shear layer on the downstream wall, and a low-pressure region at the mid-depth $(y / D=-0.4$, approximately $)$, which is associated with the vortex core. However, the second figure shows only a very small high-pressure region associated with shear layer impingement, and indicates a considerably higher position of the vortex core. This implies that the shear layer is much higher and barely impinges upon the downstream wall of the cavity at this spanwise position, as indicated by Figure 5-66. An important feature observed in this figure is the formation of a high-pressure region near the cavity floor and its downstream wall. The gradual pressure rise towards the downstream part of the cavity is verified by Figure 5-78-(a), which shows contours of pressure on the cavity base. This high-pressure region poses a resistance against entrainment of the shear layer into the cavity, and leads to formation of the vortex core at a higher position.

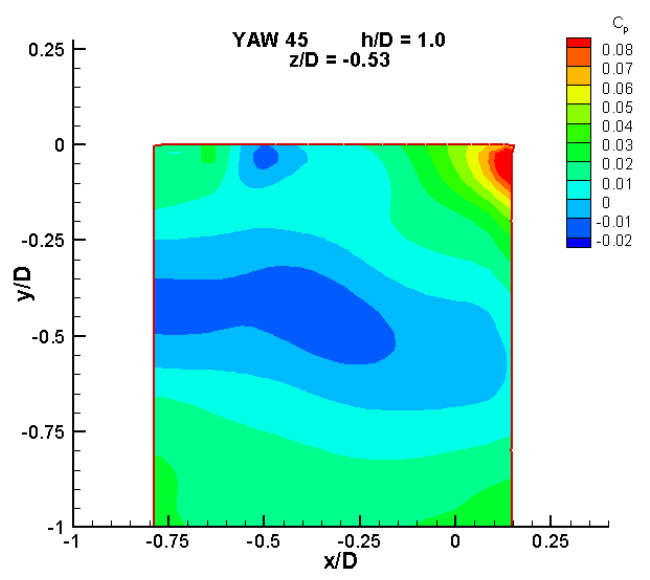

(a)

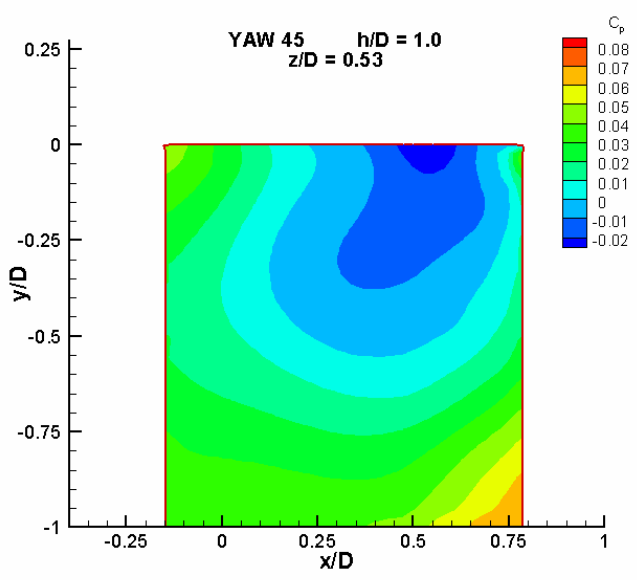

(b)

Figure 5-77 $C_{p}$ distribution at two vertical planes inside the cavity

The formation of the high pressure region in the downstream part of the cavity can be explained through examination of the flow pattern inside the cavity. Figure 5-78-(b) to (d) shows velocity vectors in horizontal planes located at $\mathrm{y} / \mathrm{D}=-0.75,-0.5$, and -0.25 , 
superimposed on the pressure contours. The flow pattern at $\mathrm{y} / \mathrm{D}=-0.25$ shows a strongly asymmetric pattern, in which a stagnation region is formed near the downstream wall at an approximate position of $x / D=-0.2$ and $z / D=-0.7$. According to Figure 5-78-(d), the flow pattern near the downstream wall is affected by a strong spanwise component (cross flow) in the positive $\mathrm{z}$ direction, which drives the major part the entrained flow towards the downstream part of the cavity.

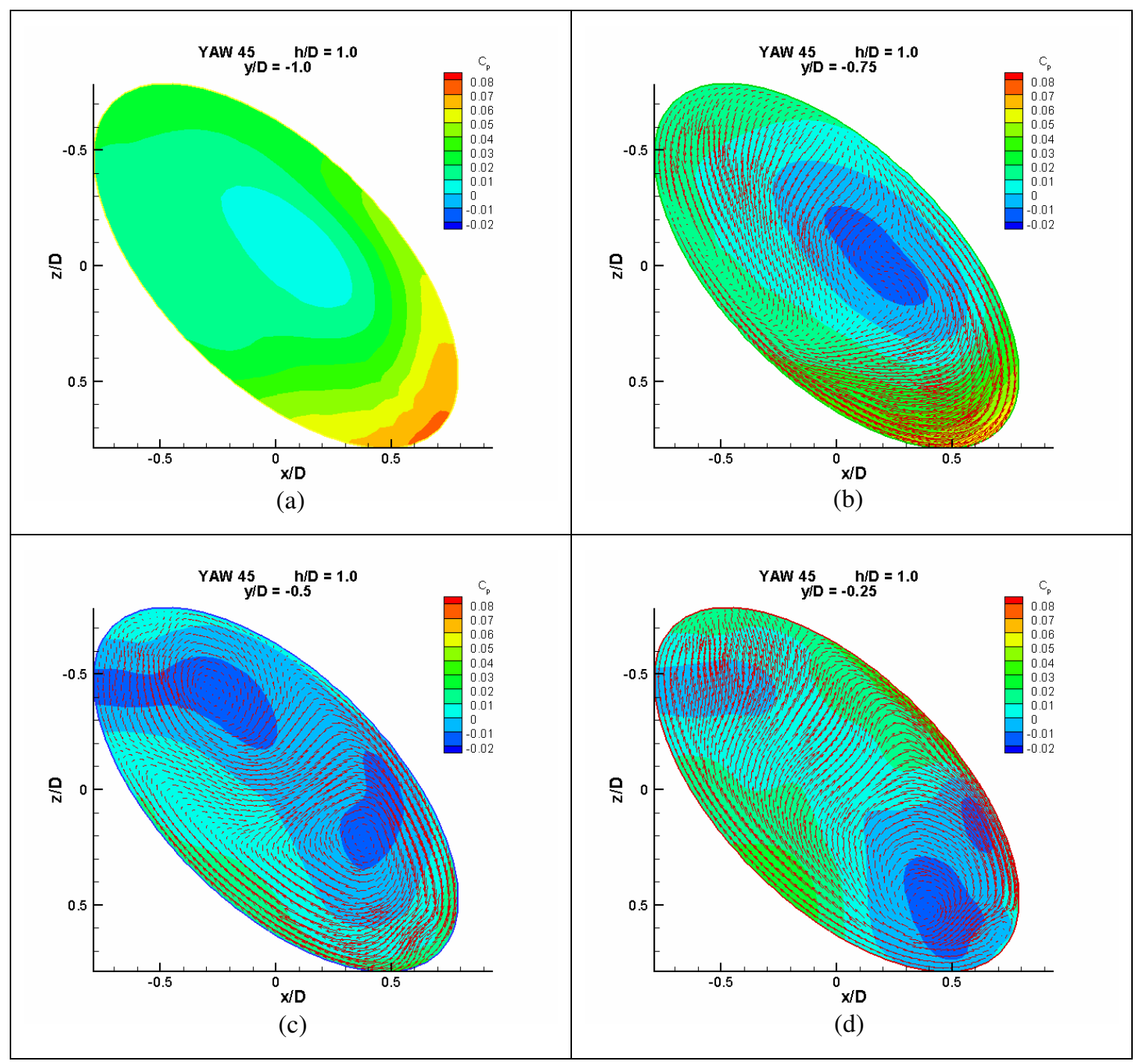

Figure 5-78 $C_{p}$ distribution contours on cavity base (a) and $C_{p}$ distribution contours and velocity vectors in three horizontal planes inside the cavity (b)-(d) 
A similar behaviour can be observed in Figure 5-78-(b) and (c) $(y / D=-0.75$ and $y / D=$ -0.5). This strong spanwise velocity component in the positive $\mathrm{z}$ direction leads to increased pressure at the downstream corner of the cavity, as indicated by the overlaid pressure coefficient contours. The asymmetric behaviour of the flow near the downstream wall, and the strong spanwise component, can be attributed to the orientation of the downstream wall with respect to the freestream, which drives the flow towards the downstream corner.

The above-mentioned process is summarized in Figure 5-79, which shows a schematic representation of the flow structure in an elliptical cavity in the asymmetric flow regime.

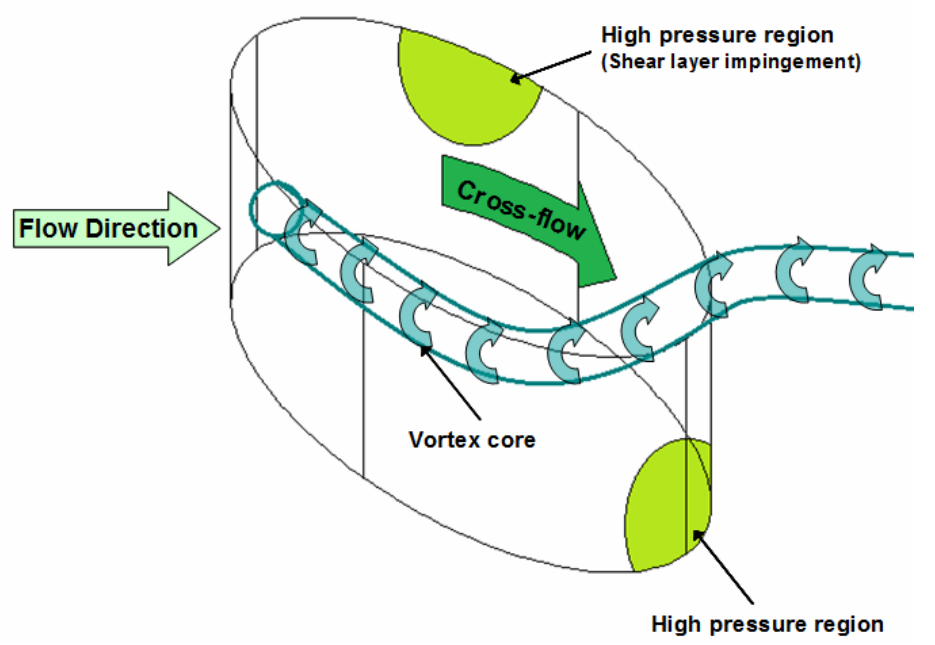

Figure 5-79 Schematic representation of the flow structure in an elliptical cavity in the strongly asymmetric flow regime

\subsubsection{Drag Characteristics}

Normalized drag coefficients $\left(\frac{\Delta C_{D}}{c_{f}}\right)$ of the elliptical cavity with $\mathrm{h} / \mathrm{D}$ ratio of 0.5 at yaw angles between 0 and $90^{\circ}$, obtained based on the present numerical simulations, are presented in Figure 5-80. These results are compared with the previously published drag data for elliptical and rectangular cavities with aspect ratio of $2: 1$ and $h / D=0.5$ in the same range of yaw angles. 
The drag values of the present study are in good agreement with those reported by Savory and Toy [5], and close to those reported by Hering [58] for elliptical cavities. Regardless of the planform geometry and upstream flow conditions, all of the data presented in Figure 5-80 indicate that the highest drag occurs at yaw angles of $45^{\circ}$ and $60^{\circ}$. In the study by Savory and Toy [5], the high drag at these yaw angles has been attributed to formation of the trailing vortices, which distinguishes the flow structure at these two yaw angles from those of the lower yaw angles. As discussed in section 5.5.2.3, formation of the trailing vortex from a yawed cavity is accompanied by formation of a secondary highpressure region on the downstream wall, immediately underneath the trailing vortex. This high-pressure region might be a contributor to the larger drag of the cavities in the strongly asymmetric regime, alongside the effect of the trailing vortex on the flow around the cavity.

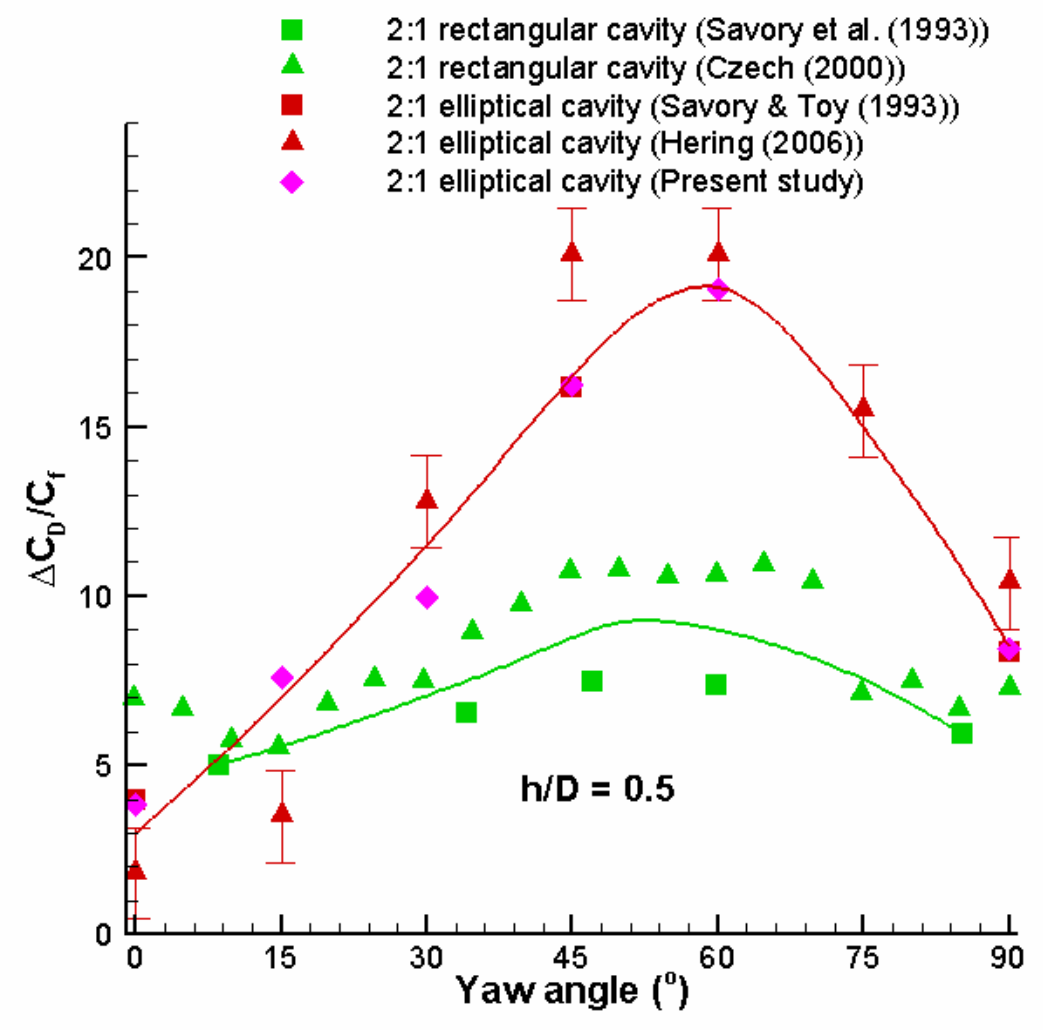

Figure 5-80 Comparison of drag coefficient for cavity with $h / D=0.5$ at various yaw angles (The lines are added to visualize the general trend for each planform shape.) 


\subsubsection{Flow Downstream of the Cavity}

As discussed in section 5.5.2.2, in the case of cavities with strongly asymmetric flow, formation of a high pressure region in the downstream part of the cavity causes the vortex core to form at a higher position, in such a way that its extreme downstream end leaves the cavity volume to form a trailing vortex. In the following paragraphs, the effect of this trailing vortex on the flow downstream of the cavity will be presented for cavities with $\mathrm{h} / \mathrm{D}=0.7,0.5$, and 0.2 at the yaw angle of $45^{\circ}$. This combination of $\mathrm{h} / \mathrm{D}$ ratios and yaw angle has been selected to make possible a direct comparison with the results reported by Hering [58].

Figure 5-81 shows the velocity deficit in vertical planes located at the positions of $\mathrm{x} / \mathrm{D}=$ 0.7 and $x / D=2.6$, based on the results of the numerical simulations of the present study, and the experiments by Hering [58]. For all three depths, the figures clearly show an area of velocity deficit at the spanwise location of the downstream corner of the cavity. To confirm that the low velocity region corresponds with the trailing vortex, the distributions of streamwise vorticity $\left(\omega_{x}\right)$ in the vertical plane located at $\mathrm{x} / \mathrm{D}=0.7$ are shown in Figure 5-82. A comparison of this figure with Figure 5-54 indicates an additional large concentration of streamwise vorticity emerging from the downstream (positive-z) half of the cavity. The location of this region corresponds with the velocity deficit regions in Figure 5-81, and the sign of vorticity matches the sense of rotation of the trailing vortex (Figure 5-79). It should be noted that positive values of $\omega_{x}$ indicate streamwise vorticity in the downstream (positive-x) direction, with circulation in clockwise direction looking downstream.

Figure 5-81 shows that the vortex core has little displacement in the spanwise direction between the measurement planes located at $x / D=0.7$ and $x / D=2.6$, suggesting that the trailing vortex becomes aligned with the flow outside the cavity. This behaviour is confirmed by Figure 5-83, which shows the velocity distribution based on PIV measurements, in a horizontal plane located $6 \mathrm{~mm}$ above the upper edge of the cavity $(\mathrm{y} / \mathrm{D}$ $=0.08$ ). The figure indicates that the low velocity region corresponding with the trailing vortex is aligned with the flow direction between $x / D=0.7$ and $x / D=2.7$. 


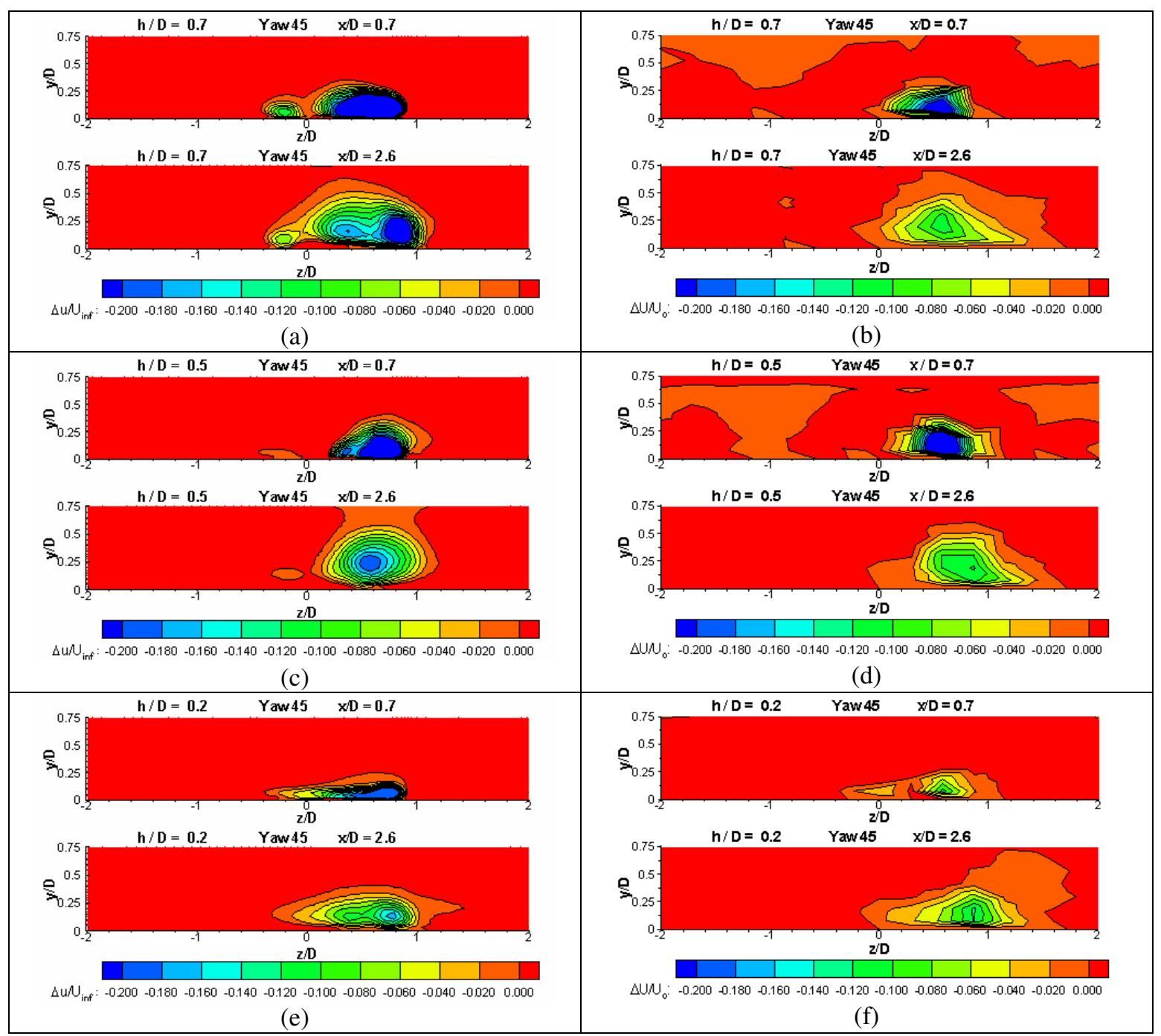

Figure 5-81 Velocity deficit contours based on numerical simulations

(left) and experimental measurements [58] (right) at $45^{\circ}$ yaw angel

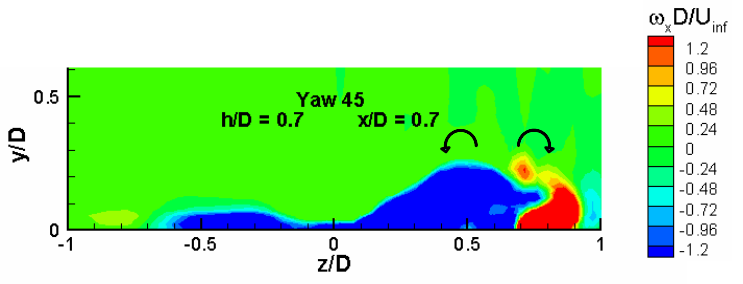

Figure 5-82 Streamwise vorticity contours at $x / D=0.7$ for $h / D=0.7$ at $45^{\circ}$ yaw angle

Also of interest is the existence of a smaller low velocity region on the opposite side of the location of the trailing vortex $(\mathrm{z} / \mathrm{D} \leq 0)$, for the cavities with $\mathrm{h} / \mathrm{D}=0.7$ and 0.5 , which is clearly visible in the results of the present numerical simulations for $h / D=0.7$ and 0.5 . 
The position of this region corresponds with that of the high pressure area associated with shear layer impingement on the downstream wall of the cavity (Figure 5-72). Therefore, it can be concluded that the low velocity region is caused by separation of the impinging shear layer from the edge of the downstream wall of the cavity, in a manner similar to that discussed in section 5.3.4 in the case of cavities with a $0^{\circ}$ yaw angle.

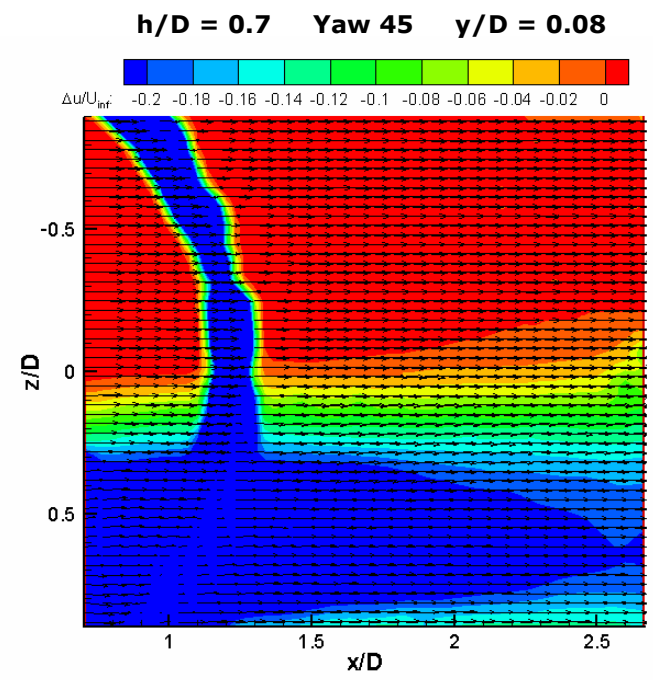

(a)

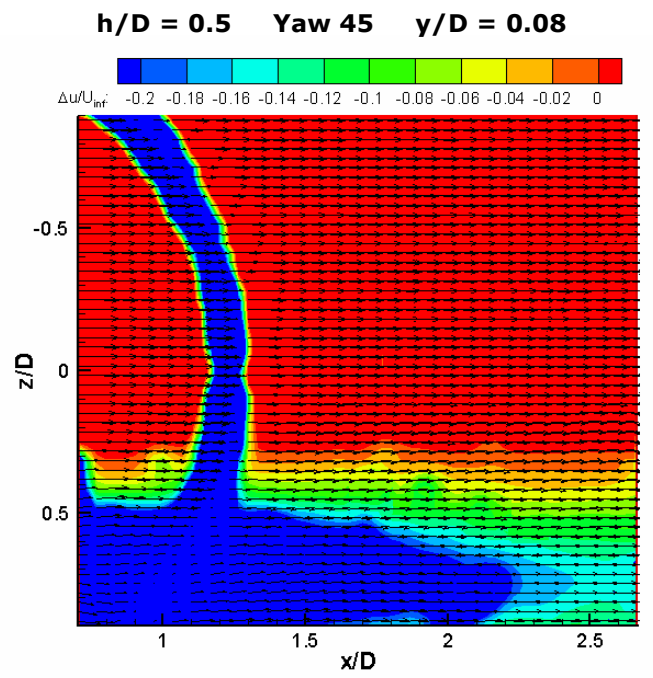

(b)

Figure 5-83 Velocity deficit contours based on PIV measurements in a horizontal plane at $45^{\circ}$ yaw angle

A quantitative comparison of the spanwise profiles of wake velocity at $\mathrm{x} / \mathrm{D}=1.5, \mathrm{y} / \mathrm{D}=$ 0.08 , based on the results of the present PIV measurements and numerical simulations is presented in Figure 5-84. Both sets of results indicate a velocity deficit $\left(\frac{\Delta \bar{u}}{U_{\mathrm{inf}}}\right)$ of -0.35 , approximately, due to the trailing vortex. This value of $\frac{\Delta \bar{u}}{U_{\mathrm{inf}}}$ is considerably larger than the values of -0.15 and -0.13 , obtained for cavities with $0^{\circ}$ and $90^{\circ}$ yaw angle, respectively, at the same downstream location (Figure 5-23 and Figure 5-53). Considering that wake velocity deficit is an indicator of the drag caused by the cavity, the larger wake velocity deficits observed in this section further confirms that the high drag of cavities in the strongly asymmetric flow regime is associated with the formation of the trailing vortex. 


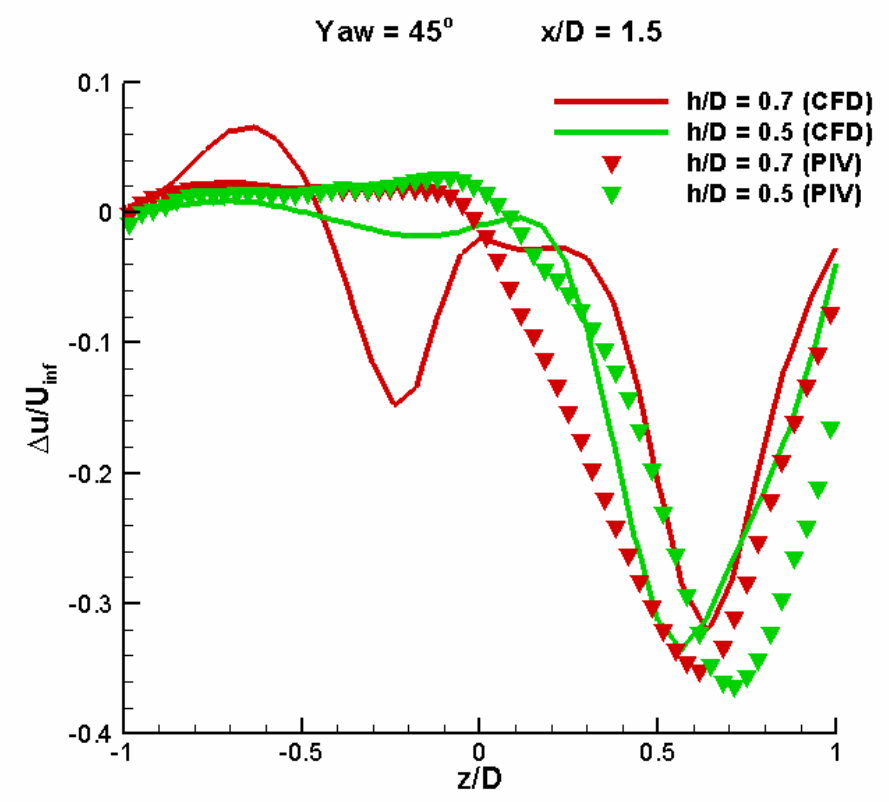

Figure 5-84 Quantitative comparison of velocity deficit at $45^{\circ}$ yaw angle

\subsubsection{Flow Dynamics}

Considering the significant effects of yaw angle on the flow structure in and around the cavity and its drag force, the dynamic characteristics of the flow are also expected to be affected. In this section, the results of the PIV velocity measurements will be analyzed to investigate the effect of the yaw angle on the dynamic characteristics of the flow inside elliptical cavities with $\mathrm{h} / \mathrm{D}=1.0$ and 0.5 , at the yaw angle of $45^{\circ}$. This yaw angle, which represents the strongly asymmetric flow regime, is selected because the flow at this yaw angle includes a trailing vortex for both $\mathrm{h} / \mathrm{D}$ ratios.

Figure 5-85 shows distributions of $u_{\mathrm{rms}}$ and $\mathrm{v}_{\mathrm{rms}}$ on a vertical line located at $\mathrm{x} / \mathrm{D}=0, \mathrm{z} / \mathrm{D}$ $=0$. The figure indicates that the effect of depth on the fluctuating velocity components is similar to that observed for the cavities at $0^{\circ}$ and $90^{\circ}$ yaw angles, in which larger fluctuating velocities were observed for smaller depths (Figure 5-26 and Figure 5-57). 


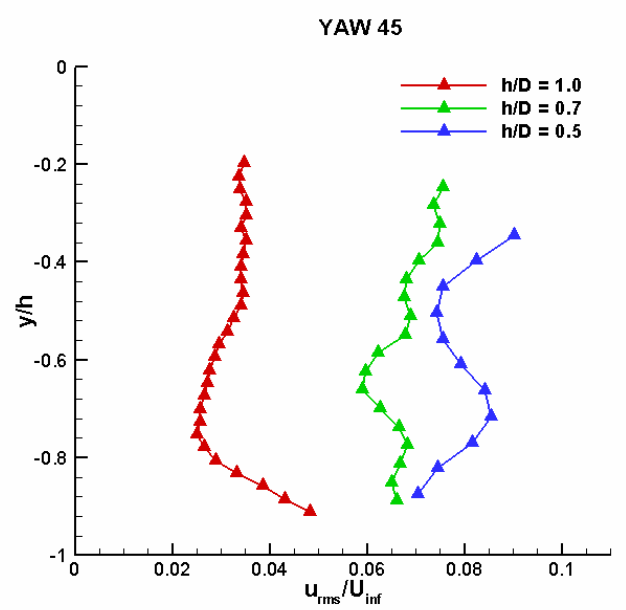

(a)

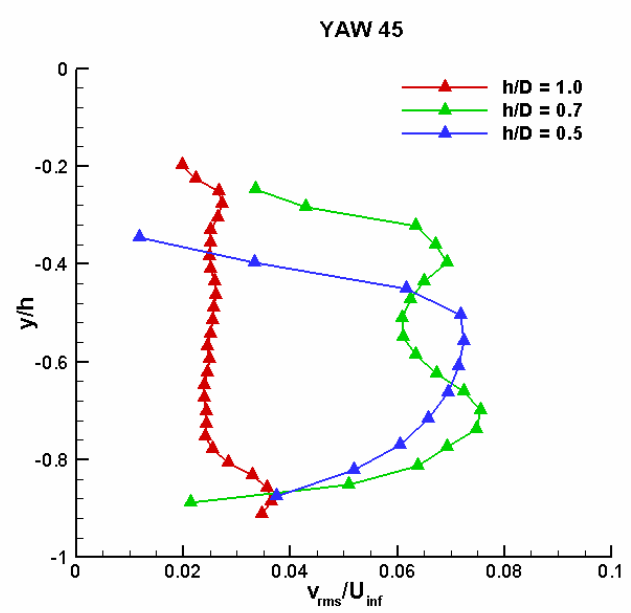

(b)

Figure 5-85 Comparison of $u_{r m s}$ (a) and $v_{r m s}$ (b) profiles on the vertical centreline

A quantitative comparison of the fluctuating velocity components with those of the cavity at $0^{\circ}$ yaw angle (Figure 5-26) indicates that the level of fluctuating velocities has not changed significantly for the cavity with yaw angle of $45^{\circ}$. This suggests that the formation of the trailing vortex does not affect the flow oscillations in the cavity midplane. The effect of the trailing vortex on the flow oscillations is expected to be more pronounced at spanwise positions closer to the trailing end of the vortex. Therefore, further analysis will be carried out in the vertical plane located at $\mathrm{z} / \mathrm{D}=0.26$, which is the farthest measurement plane from the cavity centre $(z / D=0)$ in which the vortex can be observed.

Similar to the case of the cavities with $0^{\circ}$ and $90^{\circ}$ yaw angles, POD analysis has been used in this section to investigate the dynamic behaviour of the flow inside the cavity.

The first four POD modes of the fluctuating streamwise and vertical velocity components in the cavity with $\mathrm{h} / \mathrm{D}=0.5$ are shown in Figure 5-86 and Figure 5-87. A pair-wise relationship between the first two modes, similar to those observed in the cases of cavities with $0^{\circ}$ and $90^{\circ}$ yaw angles, can be observed in these figures, suggesting that Modes 1 and 2 point to the same dynamic behaviour in the flow and are temporally related. The relative energies $\left(\lambda_{i} / \sum_{i=1}^{n} \lambda_{i}\right)$ of the first forty POD modes are shown in 
Figure 5-88 for the cavities with $\mathrm{h} / \mathrm{D}=1.0$ and 0.5 , and indicate that the first two POD modes contain $15.9 \%$ and $32.8 \%$ of the total energy of the velocity field, for $h / D=1.0$ and 0.5 , respectively. Similar to the cases of the cavities with $0^{\circ}$ and $90^{\circ}$ yaw angles (sections 5.3.5 and 5.4.5), these ratios are smaller that the typical values reported by Murray et al. [25] and Savory et al. [35] because a major part of the shear layer, which contains the highest levels of velocity fluctuations, is not included in the results of the present PIV measurements due to the masking caused by the base plate.

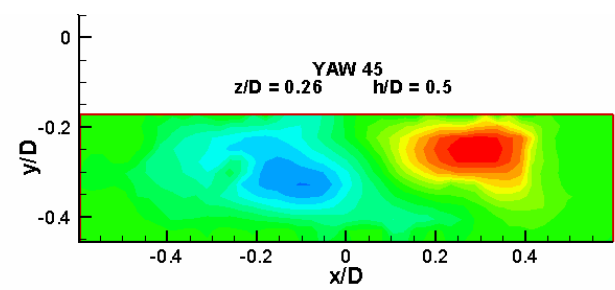

(a)

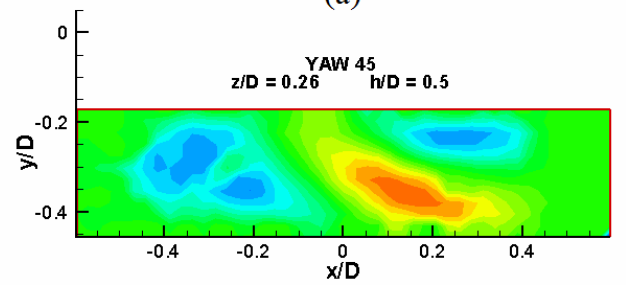

(c)

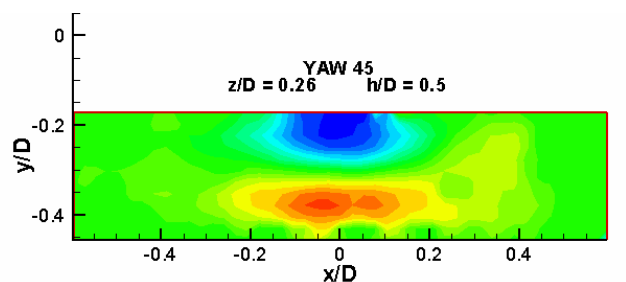

(b)

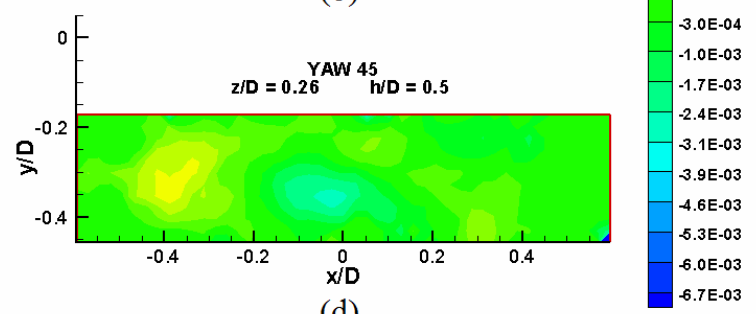

(d)

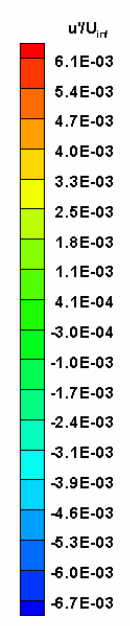

Figure 5-86 The first four POD modes of the fluctuating streamwise velocity component

Plots of normalized time-varying coefficients $a_{1}(t) / \sqrt{2 \lambda_{1}}$ vs. $a_{2}(t) / \sqrt{2 \lambda_{2}}$, which are shown in Figure 5-89, confirm the temporal relationship between the two modes, and suggests a 90-degree phase difference, as previously described in the case of the cavities with $0^{\circ}$ and $90^{\circ}$ yaw angles (sections 5.3.5 and 5.4.5). This relationship has been utilized to determine the phase angle $\theta$ for each PIV snapshot using equation (Equation 5-2), which is then used to calculate phase averaged velocity fields, in an approach similar to that implemented by Savory et al. [35].

Plots of streamtraces in the cavity based on the phase averaged velocity fields are shown in Figure 5-90 and Figure 5-91 for h/D = 1.0 and 0.5 respectively. The plots indicate vortex oscillations inside the cavity for both $\mathrm{h} / \mathrm{D}$ ratios. 


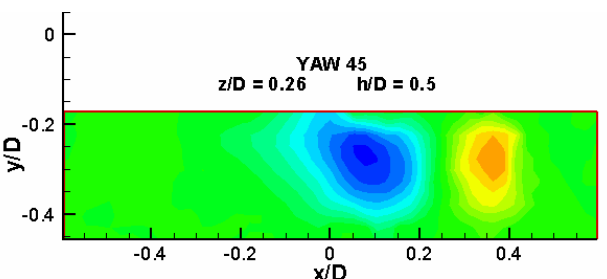

(a)

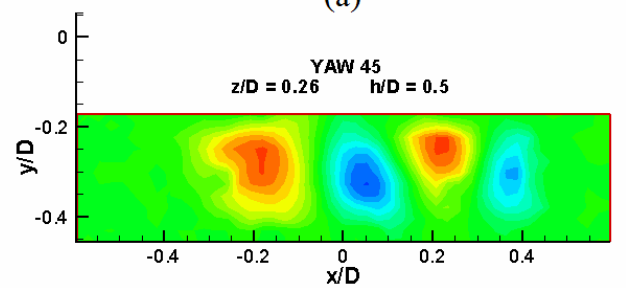

(c)

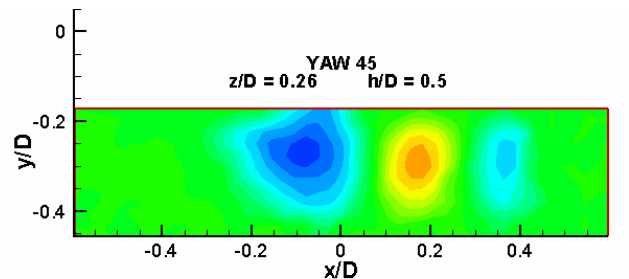

(b)

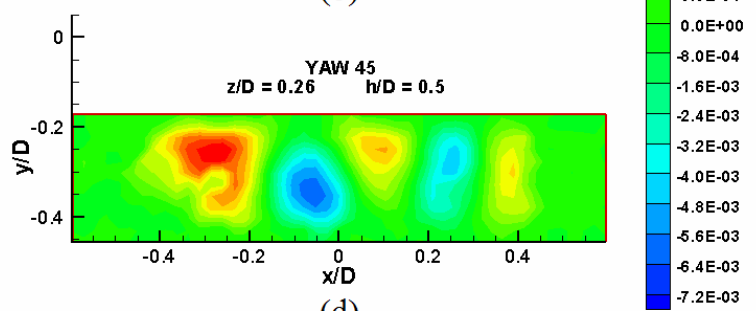

(d)

Figure 5-87 The first four POD modes of the fluctuating vertical velocity component

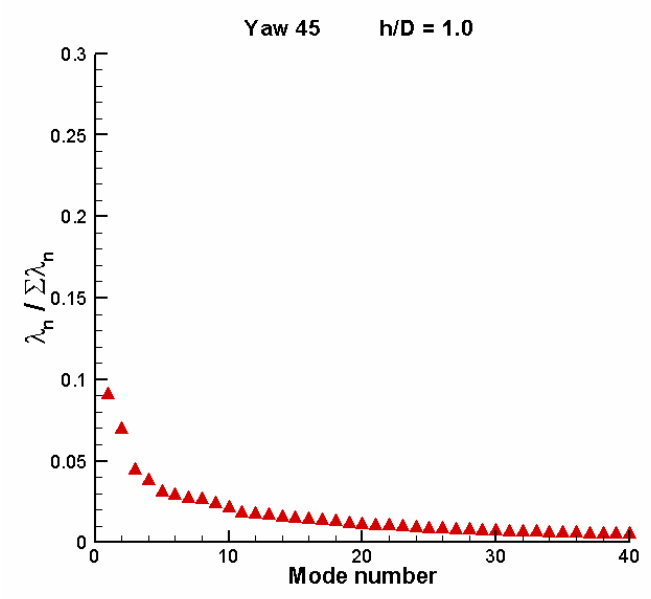

(a)

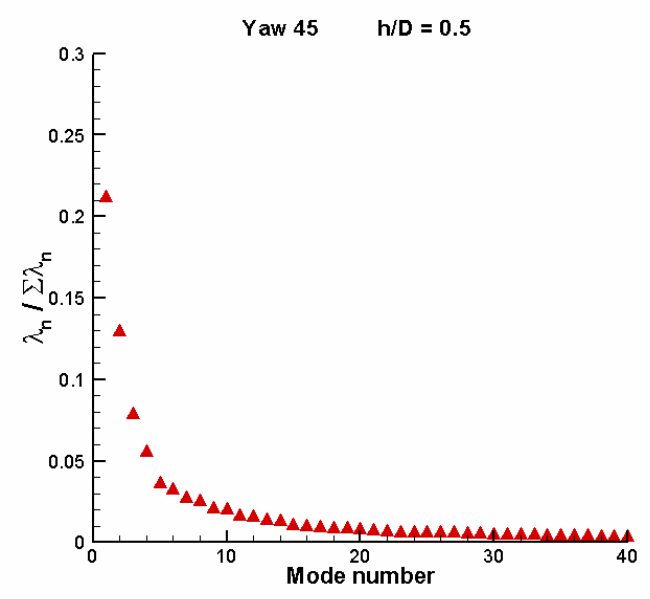

(b)

Figure 5-88 The first forty normalized eigenvalues for $\mathrm{h} / \mathrm{D}=$

1.0 (a) and 0.5 (b) at $45^{\circ}$ yaw angle at $\mathrm{z} / \mathrm{D}=0.26$

The amplitudes of oscillations in the position of the vortex core, obtained based on the phase averaged plots shown in these figures are summarized in Table 5-2, in comparison with those of the same cavity at $0^{\circ}$ yaw angle (section 5.3.5). For the cavity with $\mathrm{h} / \mathrm{D}=$ 0.5 , variations in the position of the vortex core in the streamwise and vertical directions are close to the values found for the same cavity at $0^{\circ}$ yaw angle. However, for the cavity with $\mathrm{h} / \mathrm{D}=1.0$ at the yaw angle of $45^{\circ}$, the vortex core is found to have significantly larger oscillations in the vertical direction, compared to the same cavity at $0^{\circ}$ yaw angle. 
The results for the cavity at $90^{\circ}$ yaw angle have not been included in the table, because vortex oscillations for the latter have been determined in the mid-plane $(\mathrm{z} / \mathrm{D}=0)$, and are not directly comparable to those of the cavities at $0^{\circ}$ and $45^{\circ}$, determined at an approximate $\mathrm{z} / \mathrm{D}$ of 0.2 .

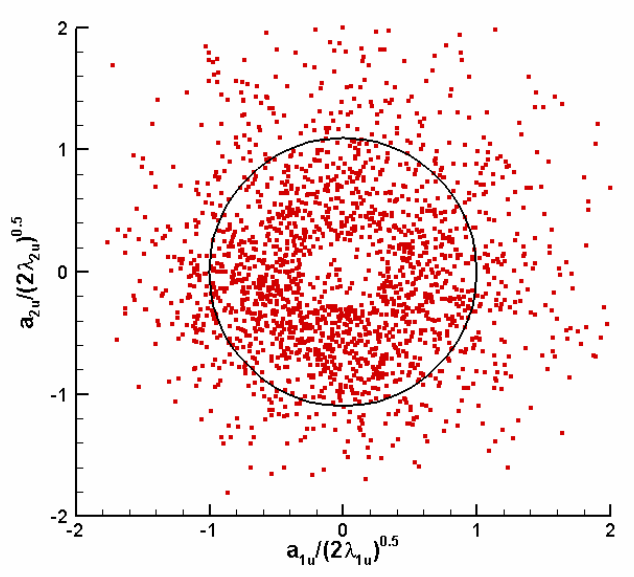

(a)

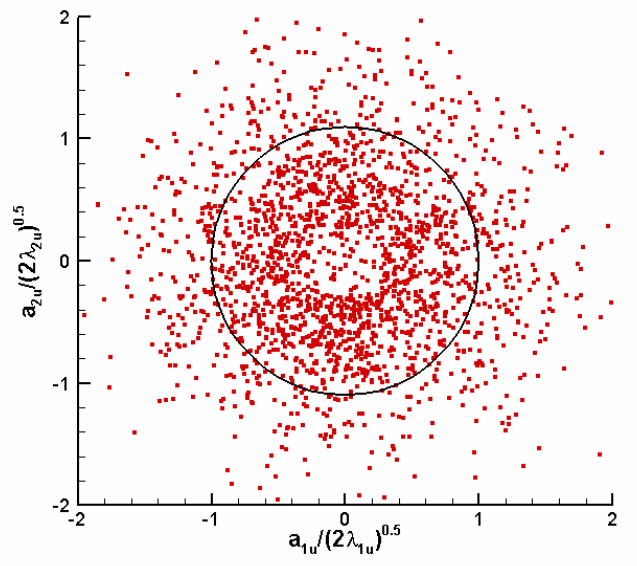

(b)

Figure 5-89 Normalized time-varying coefficients for the cavities with $h / D=1.0$ (a) and 0.5 (b)

Considering the relatively large amplitude of the oscillations, the trailing vortex is expected to cause an increase in the fluctuating velocity components in the regions of the flow downstream of the cavity that are influenced by it. This effect can be verified through a quantitative comparison of the fluctuating velocity components in the flow downstream of the cavity at various yaw angles. Such a comparison is presented in Figure 5-92 for the cavity with $\mathrm{h} / \mathrm{D}=0.5$ at yaw angles of $0^{\circ}, 45^{\circ}$, and $90^{\circ}$ at $0.1 \mathrm{D}$ downstream of the cavity. The profiles have different shapes, due to different flow phenomena affecting flow in the wake (downstream edge separation for yaw $=0^{\circ}$, the trailing vortex for yaw $=45^{\circ}$, and streamwise vortices at yaw $=90^{\circ}$ ). However, the figure indicates that the trailing vortex generates the highest level of streamwise velocity fluctuations (represented by $\mathrm{u}_{\mathrm{rms}}$ ) among all cases. 


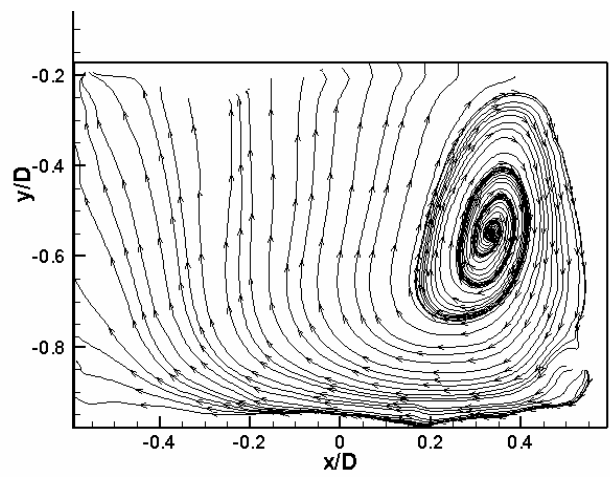

(a)

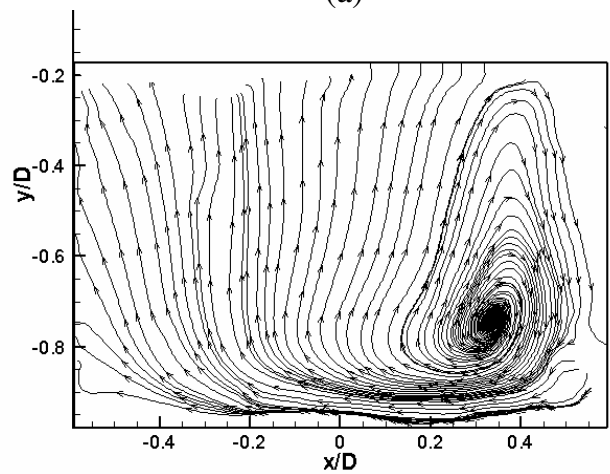

(c)

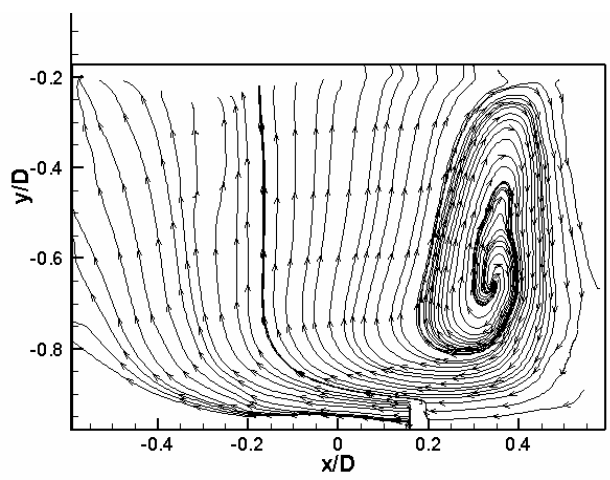

(b)

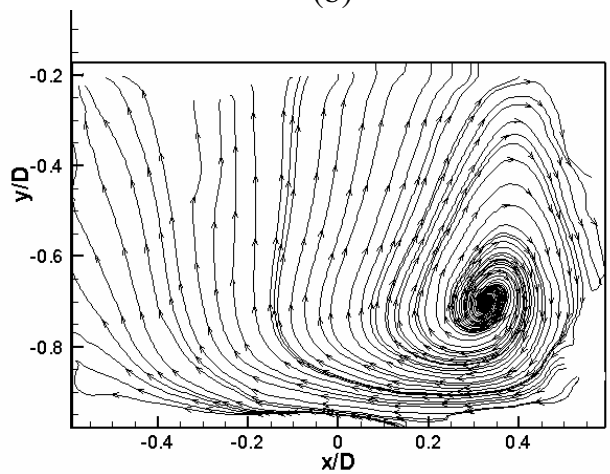

(d)

Figure 5-90 Phase averaged streamtraces in the vertical plane located at $\mathrm{z} / \mathrm{D}=\mathbf{0 . 2 6}$,

for the cavity with $h / D=1.0$. The phase interval between consecutive plots is $90^{\circ}$

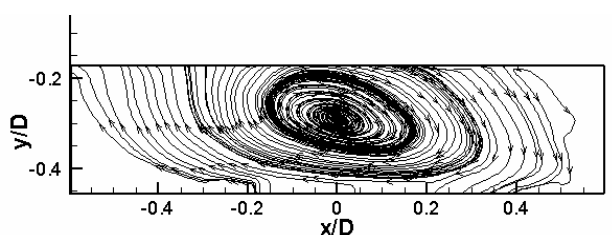

(a)

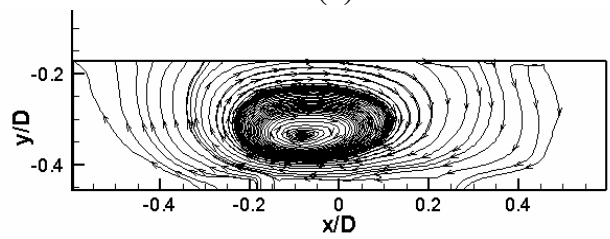

(c)

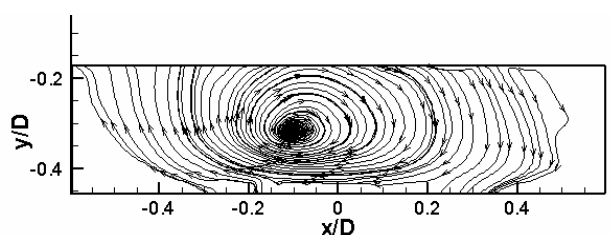

(b)

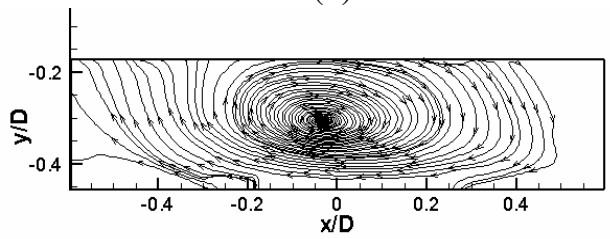

(d)

Figure 5-91 Phase averaged streamtraces in the vertical plane located at $\mathrm{z} / \mathrm{D}=\mathbf{0 . 2 6}$, for the cavity with $h / D=0.5$. The phase interval between consecutive plots is $90^{\circ}$ 
Table 5-2 Amplitude of oscillations of the vortex core based on the phase-averaged streamtraces

\begin{tabular}{|c|c|c|c|c|}
\hline & \multicolumn{3}{|c|}{ Amplitude of oscillations } \\
\hline Yaw angle & \multicolumn{2}{|c|}{$0^{\circ}$} & \multicolumn{2}{c|}{$45^{\circ}$} \\
\hline Direction & $x$ & $y$ & $x$ & $y$ \\
\hline h/D = 1.0 & $0.03 \mathrm{D}$ & $0.04 \mathrm{D}$ & $0.02 \mathrm{D}$ & $0.21 \mathrm{D}$ \\
\hline h/D = 0.5 & $0.07 \mathrm{D}$ & $0.10 \mathrm{D}$ & $0.09 \mathrm{D}$ & $0.10 \mathrm{D}$ \\
\hline
\end{tabular}

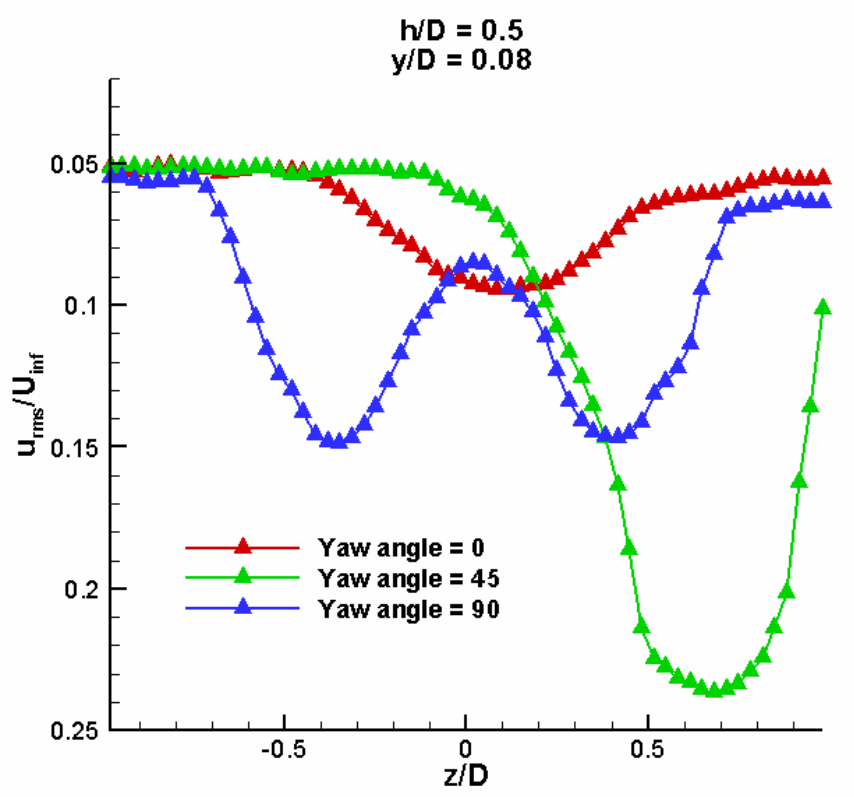

Figure 5-92 Comparison of $u_{\text {rms }}$ values for three different yaw angles at

0.1D downstream of the cavity with $h / D=0.5$ and $y / D=0.08$

\subsubsection{Summary of Findings for the Asymmetric Flow Regimes}

In this section, the results of the PIV measurements and numerical simulations of present study were analyzed in comparison with previously published results, to determine the features of the flow in and around yawed cavities. Two flow regimes were studied: the weakly asymmetric regime, which includes cavities at yaw angles of $15^{\circ}$ and $30^{\circ}$, and the 
strongly asymmetric regime, which includes cavities at yaw angles of $45^{\circ}$ and $60^{\circ}$. The findings presented in this section can be summarized as follows:

- Flow asymmetry has significant effects on the separated shear layer, such that the behaviour of the separated shear layer for yawed cavities cannot be directly associated with those of nominally two-dimensional rectangular cavities, in "open", "closed" and "transitional" regimes. In the weakly asymmetric flow regime, the growth rate of the separated shear layer is similar to that of symmetric flow regimes $\left(d \delta_{\omega} / d x=0.2\right)$. However, in the strongly asymmetric regime, the shear layer growth is non-linear, with rates larger than the above-mentioned typical value.

- In the weakly asymmetric flow regime, the flow structure in the cavity with any $\mathrm{h} / \mathrm{D}$ ratio preserves the features of the flow structure in the same cavity at zero yaw angle, with slight asymmetric effects. The cavities in the strongly asymmetric regime, however, show flow structures that cannot be directly associated with those of the similar cavities at zero yaw angle.

- The most prominent feature of the flow structure in asymmetric regimes is the vertical displacement of the vortex towards the upper edge, in the downstream part of the cavity. This feature is attributed to formation of a high-pressure region at the downstream corner of the cavity, due to the cross flow along the downstream wall.

- In the strongly asymmetric flow regime, the vertical displacement of the downstream part of the vortex reaches the extent that causes the vortex to exit the cavity volume and form a trailing vortex. This behaviour is observed at all h/D ratios, including $\mathrm{h} / \mathrm{D}=1.0$, which is typically associated with the "deep" flow regime involving a "captive" vortex.

- The cavities in the strongly asymmetric regime (yaw $=45^{\circ}$ and $60^{\circ}$ ) have the largest drag among all of the cases studied herein. The secondary high-pressure 
region formed on the downstream wall of the cavity is believed to be a contributor to the high drag.

- The trailing vortex causes a low velocity region in the flow downstream of the cavity, with a velocity deficit that is larger than those observed downstream of similar cavities in symmetric flow regimes. This large velocity deficit is consistent with the high drag of the cavities in the strongly asymmetric regime.

- In cavities in the strongly asymmetric regime, the downstream part of the vortex is found to have large oscillations within the cavity volume, these oscillations influence the flow downstream of the cavity, and lead to velocity fluctuations larger than those observed downstream of cavities with symmetric flows.

- A transition in the general orientation of the vortex in the cavity with $h / D=1.0$ is found to occur at the yaw angle of $60^{\circ}$. At this yaw angle, the vortex, which is generally aligned with the major axis of the cavity at lower yaw angles, re-aligns with the minor axis, similar to the case of the cavity at $90^{\circ}$ yaw angle. This transition is not observed for cavities with smaller depths, however, Savory et al. [48] have mentioned the possibility of a similar transition based on their observations of the flow in a rectangular cavity with an aspect ratio of 2:1.

\subsection{Summary}

In this chapter, the results of experimental measurements and numerical simulations of the flow inside and around cavities with 2:1 elliptical planforms were presented and analyzed for various depths and yaw angles.

The analysis involved a combination of the present experimental and numerical results. In order to use this combination, quantitative and qualitative agreement between these sources of data were established in section 5.2, through comparative evaluation of the results.

Cavity flows were categorized into the following general flow regimes, which were defined considering yaw angle as a key parameter: 
- The symmetric flow regimes, which include cavities at zero yaw angle (section 5.3)

- The nominally-two-dimensional flow regime, which included cavities at $90^{\circ}$ yaw angle (section 5.4)

- The asymmetric flow regimes, which include cavities with non-zero yaw angles between $15^{\circ}$ and $60^{\circ}$ (section 5.5).

The investigation of the flow in each flow regime included deep, intermediate-depth and shallow cavities, with $\mathrm{h} / \mathrm{D}$ ratios between 1.0 and 0.1 . For each of the three general flow regimes, the following aspects of the flow inside and around the cavity were investigated:

- The principal parameters of the shear layer and their relationship with the cavity flow structure

- The three-dimensional flow structure in the cavity, determined using a combination of the velocity vector field and vortex core positions based on PIV measurements and surface pressure distributions based on numerical simulations

- The drag of the cavity and its relationship with the flow structure

- The features of the flow downstream of the cavity and their relationship with the cavity flow structure

- The dynamic behaviour of the flow inside the cavity

In each case, the results were compared to those reported for similar elliptical cavities, as well as three-dimensional and nominally two-dimensional rectangular cavities, and similarities and differences were highlighted.

To enable the reader to follow the chapter more easily, the key findings regarding each of the three flow regimes are summarized in the last part of the respective section (sections 5.3.6, 5.4.6, and 5.5.6) and will not be repeated here. 
These findings will be discussed in detail in the conclusions of the thesis (Chapter 6). By comparing and combining the findings for all three flow regimes, the hypotheses and the objectives of the research will be addressed, and a modified version the general categorization of the flow regimes, shown in Figure 5-1, will be proposed. 


\section{Conclusions and Recommendations}

\subsection{Summary of the Findings}

In the present research, the flow inside and around three-dimensional cavities with a 2:1 aspect ratio elliptical planform, exposed to a turbulent upstream boundary layer has been studied, for several combinations of cavity depth and yaw angle. A combination of wind tunnel experiments and computational fluid dynamics simulations were used as the principal tools for this study. The results of the numerical simulations were evaluated through qualitative and quantitative comparisons with the experimental results, and it was found that steady numerical simulations with the Reynolds Stress Model as the turbulence closure approach, can be used as a cost-effective tool for prediction of mean flow parameters.

To carry out a systematic investigation of the effects of cavity depth and yaw angle, cavity flows have been categorized into the following general flow regimes, which are defined considering yaw angle as a key parameter:

- Symmetric flow regimes: These flow regimes are associated with shallow, intermediate-depth, and deep cavities at $0^{\circ}$ yaw angle. The present study includes cavity depth to minor axis (h/D) ratios of $0.1,0.2,0.35,0.5,0.7$, and 1.0.

- Nominally two-dimensional flow regime: This flow regime is associated with cavities at the yaw angle of $90^{\circ}$. Three $\mathrm{h} / \mathrm{D}$ ratios of $0.1,0.5$, and 1.0 have been covered in the present study, which represent the shallow, intermediate depth, and deep cavities.

- Asymmetric flow regimes: These flow regimes are divided into two subcategories: The weakly asymmetric regime, which is associated with cavities with yaw angles up to $30^{\circ}$, and the strongly asymmetric regime, which is associated with cavities with yaw angles of $45^{\circ}$ and $60^{\circ}$. For each of the two sub-categories, three $\mathrm{h} / \mathrm{D}$ ratios of $0.1,0.5$, and 1.0 , which represent the shallow, intermediate depth, and deep cavities have been studied. 
In accordance with the objectives specified in Chapter 1, the following aspects of the flow inside and around the cavity have been investigated in each flow regime. Based on the results presented in Chapter 5, the key findings with regards to each of these aspects can be summarized as follows:

\section{- The separated shear layer}

For cavities with all $\mathrm{h} / \mathrm{D}$ ratios in the symmetric, nominally two-dimensional, and weakly asymmetric flow regimes, the shear layer grows almost linearly downstream of the

separation point, with an average growth rate of $d \delta_{\omega} / d x=0.2$, approximately, which is within the range of the growth rates observed for nominally two-dimensional rectangular cavities.

In the strongly asymmetric flow regime (yaw angles of $45^{\circ}$ and $60^{\circ}$ ), the growth rate is found to be non-linear and larger than other cases, due to strong effects of yaw angle on the flow structure inside the cavity and the vertical position of the vortex inside of these cavities.

In the symmetric and nominally two-dimensional flow regimes, the behaviour of the shear layer, represented by the position of the shear layer centre along the upper edge of the cavity, is similar to that of a nominally two-dimensional rectangular cavity with the same $\mathrm{h} / \mathrm{D}$ ratio.

For intermediate-depth cavities $(0.2 \leq \mathrm{h} / \mathrm{D} \leq 0.7)$ in the symmetric flow regime (yaw angle $=0^{\circ}$ ), the behaviour of the shear layer cannot be directly associated with the respective nominally two-dimensional rectangular cavities. This regime includes formation of cellular flow structures in the cavity.

In asymmetric flow regimes $\left(15^{\circ} \leq\right.$ yaw angle $\left.\leq 60^{\circ}\right)$ the position of the shear layer centre is found to be influenced by the vertical displacement of the vortex, which increases towards the downstream corner of the cavity and so the flow cannot be associated with that of nominally two-dimensional rectangular cavities. 


\section{- The flow structure}

\section{$\bigcirc \quad$ The symmetric flow regime (yaw angle $=0^{\circ}$ )}

Three distinct flow structures are found to exist, depending on $\mathrm{h} / \mathrm{D}$ ratio: The flow structure for the cavity with $\mathrm{h} / \mathrm{D}=1.0$ consists of a single, captive vortex similar to that of deep nominally two-dimensional cavities in the "open" regime. For cavities with $\mathrm{h} / \mathrm{D}$ ratios between 0.7 and 0.35 , the flow structure includes a single, captive vortex showing a cellular structure with two cells. This phenomenon has been observed for cavities with rectangular planforms, however, the aspect ratio at which it initiates is larger, and no cellular structures are observed in rectangular cavities with 2:1 aspect ratio [48] and [49]. For cavities with h/D ratios of 0.2 and 0.1 , the flow structure resembles that of shallow nominally two-dimensional cavities in the "transitional" flow regime.

\section{$\circ \quad$ The nominally two-dimensional flow regime (yaw angle $=90^{\circ}$ )}

The flow structure for the cavities with $\mathrm{h} / \mathrm{D}=1.0$ and 0.5 consists of a captive vortex similar to that of deep nominally two-dimensional cavities in the "open" regime. The vortex position and orientation shows little variation across the span. For the cavity with $\mathrm{h} / \mathrm{D}=0.1$, the flow structure resembles that of shallow nominally twodimensional cavities in the "closed" flow regime.

While the flow structure is found to be similar to that of nominally two-dimensional rectangular cavities in general, certain differences are found to exist, due to the effects of wall curvature. For example, for the cavities with $\mathrm{h} / \mathrm{D}=1.0$ and 0.5 , the primary vortex is formed further downstream compared to nominally twodimensional rectangular cavities, and the secondary vortex is either very small, or non-existent. For the cavity with $\mathrm{h} / \mathrm{D}=0.1$, the vortex core is found to be curved and parallel to the upstream wall. Considering these differences, the term "nominally twodimensional" may not be an adequate term to describe this flow regime. 


\section{$\circ$ The asymmetric flow regimes $\left(15^{\circ} \leq y a w \leq 60^{\circ}\right)$}

In the weakly asymmetric flow regime, the flow structure in the cavity with any $\mathrm{h} / \mathrm{D}$ ratio preserves the features of the flow structure in the same cavity at $0^{\circ}$ yaw angle, with slight asymmetric effects. The cavities in the strongly asymmetric regime, however, show flow structures that cannot be directly associated with those of the similar cavities at $0^{\circ}$ yaw angle.

The most prominent feature of the flow structure in asymmetric regimes is the vertical displacement of the vortex towards the upper edge, in the downstream part of the cavity. This feature is attributed to formation of a high-pressure region at the downstream corner of the cavity, due to the cross flow along the downstream wall (Figure 5-79).

In the strongly asymmetric flow regime, the vertical displacement of the downstream part of the vortex reaches the extent that causes the vortex to exit the cavity volume and form a trailing vortex. In the present study, this behaviour is observed for $\mathrm{h} / \mathrm{D}$ ratios of 1.0 and 0.5 . Therefore, the flow structure in cavities with $\mathrm{h} / \mathrm{D}=1.0$ at yaw angles of $45^{\circ}$ and $60^{\circ}$ cannot be categorized as "deep", which is typically associated a "captive" vortex.

A transition in the general orientation of the vortex in the cavity with $h / D=1.0$ is found to occur at the yaw angle of $60^{\circ}$. At this yaw angle, the vortex, which is generally aligned with the major axis of the cavity at lower yaw angles, re-aligns with the minor axis, similar to the case of the cavity at $90^{\circ}$ yaw angle.

For the shallow cavity $(\mathrm{h} / \mathrm{D}=0.1)$ in the asymmetric flow regime, the effect of yaw angle on the flow structure is minimal, and the vortex is found to remain normal to the freestream at all yaw angles. 


\section{- The drag of the cavity}

For cavities in the symmetric and nominally two-dimensional regimes, the drag is found to be lower for cavities with larger h/D ratios (deeper cavities). This behaviour has been attributed to the increased entrainment of the shear layer in the case of shallow and transitional cavities.

The cavities in the strongly asymmetric regime (yaw $=45^{\circ}$ and $60^{\circ}$ ) have the largest drags among all of the cases studied herein. This high drag has been related in previous studies to the formation of the trailing vortex. Based on the flow structure determined in the present study for this flow regime (Figure 5-79), the secondary high-pressure region formed on the downstream wall of the cavity is also believed to be a contributor to the high drag.

Drag variations due to changes in cavity depth and yaw angle, found in the present study, are similar in trend to those reported in previous studies involving elliptical cavities and rectangular cavities [3], [5], [48], [49], and [58].

\section{- The flow downstream of the cavity}

In all three flow regimes, the flow downstream of the cavity is found to be directly influenced by the flow structure within the cavity.

For the cavities in the symmetric flow regime, a single region of velocity deficit is found to exist on the centreline downstream of the cavity, for all depths. This is due to separation of the flow from the downstream edge of the cavity. The velocity deficit for the cavity with $h / D=0.5$ is found to be larger than of $h / D=0.7$, which is consistent with the higher drag for this $\mathrm{h} / \mathrm{D}$ ratio.

For the cavities in the nominally two-dimensional flow regime, the flow downstream of the cavity has been found to be affected by a pair of streamwise vortices originating from the cavity. The mechanism of formation and the sense of rotation of these vortices are 
different from the side edge vortices, generated by a rectangular cavity and are related to separation of flow from the curved downstream edge of the elliptical cavity.

For the cavities in the asymmetric flow regime, the trailing vortex causes a low velocity region in the flow downstream of the cavity, with a velocity deficit that is larger than those observed downstream of similar cavities in symmetric and nominally twodimensional flow regimes. This large velocity deficit is consistent with the high drag of the cavities in the strongly asymmetric regime.

\section{- The fluctuating velocities and vortex oscillations}

For cavities in the symmetric and nominally two-dimensional flow regimes, the flow in deeper cavities is found to be more stable that in the shallower ones. This is indicated by lower levels of fluctuating velocity components and smaller amplitudes of oscillations of the vortex core inside the cavity for deeper cavities. This behaviour is consistent with the observations reported by Hering [48] and Ukeiley and Murray [26]. In the case of $90^{\circ}$ yaw angle, velocity fluctuations and vortex oscillations are found to be generally larger than those of cavities with $0^{\circ}$ yaw angle, due to larger length of the cavity in the streamwise direction, which allows increased growth of the fluctuations in the shear layer. In previous studies (Haigermoser et al. [24] and Ukeiley and Murray [26]) similar oscillations of the vortex have been attributed to flapping of the shear layer, due to an instability which is independent of the resonant conditions, which leads to in- and outflow events (known as breathing). Although the present PIV measurements do not allow an investigation of the dynamics of the shear layer itself due to masking of the images, similar vertical oscillations of the vortex, and the fact that the present cavity is not in resonant conditions (section 2.5), suggests that a similar flapping instability in the shear layer is responsible for the vortex oscillations.

In cavities in the strongly asymmetric regime, the downstream part of the vortex is found to have large oscillations within the cavity volume. Unlike cavities in the symmetric and nominally two-dimensional regimes, the vortex oscillations have a larger amplitude for the cavity with $\mathrm{h} / \mathrm{D}=1.0$ in this regime. These oscillations influence the flow 
downstream of the cavity and lead to velocity fluctuations larger than those observed downstream of cavities with symmetric flows.

\subsection{Conclusions}

These findings can be used to evaluate the hypotheses of the present research, described in Chapter 1. The following conclusions can be made by comparing the above-mentioned findings with the hypotheses:

- Three-dimensional effects due to the curvature of sidewalls: The findings of the present work shown that the curvature of the sidewalls leads to additional threedimensional effects in the flow inside and around cavities. Some of these effects, which have been found in the present study, include formation of curved recirculation zones parallel to the curved walls of the cavity, and formation of streamwise vortices upon separation of the flow from the curved downstream edge of the cavity. These effects are most pronounced for cavities at $90^{\circ}$ yaw angle, where the walls facing the free stream have the largest curvature.

- Dependence of flow regime on cavity depth: The present research has shown that the flow regime in elliptical cavities is dependent on the cavity depth. However, the flow structures in the regimes occurring at various depths are sometimes different from those of rectangular and nominally two-dimensional cavities. An example of these differences is formation of cellular structures in the cavity with aspect ratio of 2:1, which does not occur for rectangular cavities.

- Asymmetric flow regimes and high drag for yawed cavities: In the present study, various asymmetric flow structures have been found to exist in elliptical cavities, depending on the combination of yaw angle and cavity depth. The dominant feature of these flow structures is the vertical displacement of the vortex towards the upper edge of the cavity in the downstream part of the cavity volume, according to the mechanism illustrated in Figure 5-79. Formation of a trailing vortex due to this behaviour is found to be associated with the high drag values at 
large yaw angles $\left(45^{\circ}\right.$ and $60^{\circ}$ ), in a manner similar to cavities with rectangular and circular planforms.

- Behaviour of the separated shear layer and dependence of the flow inside the cavity on it: Results of the present work indicate that except for the cases where interference occurs due to departure of the vortex from the cavity volume, the shear layer grows linearly upon separation from the upstream edge of the cavity, with a growth rate that is independent of cavity depth and yaw angle. The shear layer is the driving mechanism of the flow inside the cavity; therefore, the quantitative values of mean and fluctuating velocity components inside the cavity are related to those of the separated shear layer, rather than the free stream.

- The influence of the flow phenomena caused by the cavity on the wake: The findings of the present work confirm that the flow in the wake of the cavity is influenced by the flow phenomena caused by the presence of the cavity. These phenomena include separation of flow from the downstream edge of the cavity and the associated streamwise vortices, and the trailing vortex formed in the asymmetric flow regimes. The largest mean velocity deficit and fluctuating velocity components in the wake are associated with the trailing vortex. The cavities with trailing vortices also have the largest drag, which is partly due to formation of a high pressure region at the downstream corner because of the cross flow caused by impingement of the shear layer on the curved downstream wall of the cavity.

- Dependence of the dynamic behaviour of the flow on the boundary layer and geometric parameters: Considering the ratio of upstream boundary layer thickness to length $(\delta / D)$ for the present cavity, and according to the criteria mentioned by Ahuja and Mendoza [44], the cavity is outside the range of feedback resonance in the present study. However, oscillations of the cavity vortex are found to exist in many combinations of cavity depth and yaw angle, possibly due to a shear layer flapping behaviour similar to those identified by 
Haigermoser [24] and Ukeiliy and Murray [26]. Larger vortex oscillations are accompanied by increased levels of velocity fluctuations in the cavity.

As mentioned at the beginning of Chapter 5, Hering and Savory [6] have proposed a categorization of the flow regimes in an elliptical cavity with an aspect ratio of 2:1, for various depths and yaw angles (Figure 5-1). This categorization, which is based on surface pressure measurements, was used in Chapter 5 as a framework for analyzing the flow in key combinations of cavity depth and yaw angle. However, the results of the present analysis show certain differences and features which were not in accordance with this earlier categorization. Specifically, the cavity with $\mathrm{h} / \mathrm{D}=1.0$, which was categorized as a "deep" cavity with a captive vortex, is found to have a trailing vortex in the strongly asymmetric flow regime. Also, the flow inside and around cavities at the yaw angle of $90^{\circ}$ is found to have features that are caused by the large curvature of the upstream and downstream walls that are not consistent with the flow in nominally two-dimensional cavities as described in detail in Section 5-4.

A modified categorization of the flow regimes in the elliptical cavity with an aspect ratio of 2:1 is proposed in Figure 6-1, to reflect the above-mentioned findings. In this modified categorization, the strongly asymmetric flow regime, which involves formation of trialing vortices, has been extended to the cavity with $\mathrm{h} / \mathrm{D}=1.0$. Also, considering that the flow in deep and intermediate-depth cavities at the yaw angle of $90^{\circ}$ contains a vortex that has very little curvature despite strong three-dimensional effects, the term "nominally twodimensional" has been replaced with "straight vortex" to better describe this regime. 


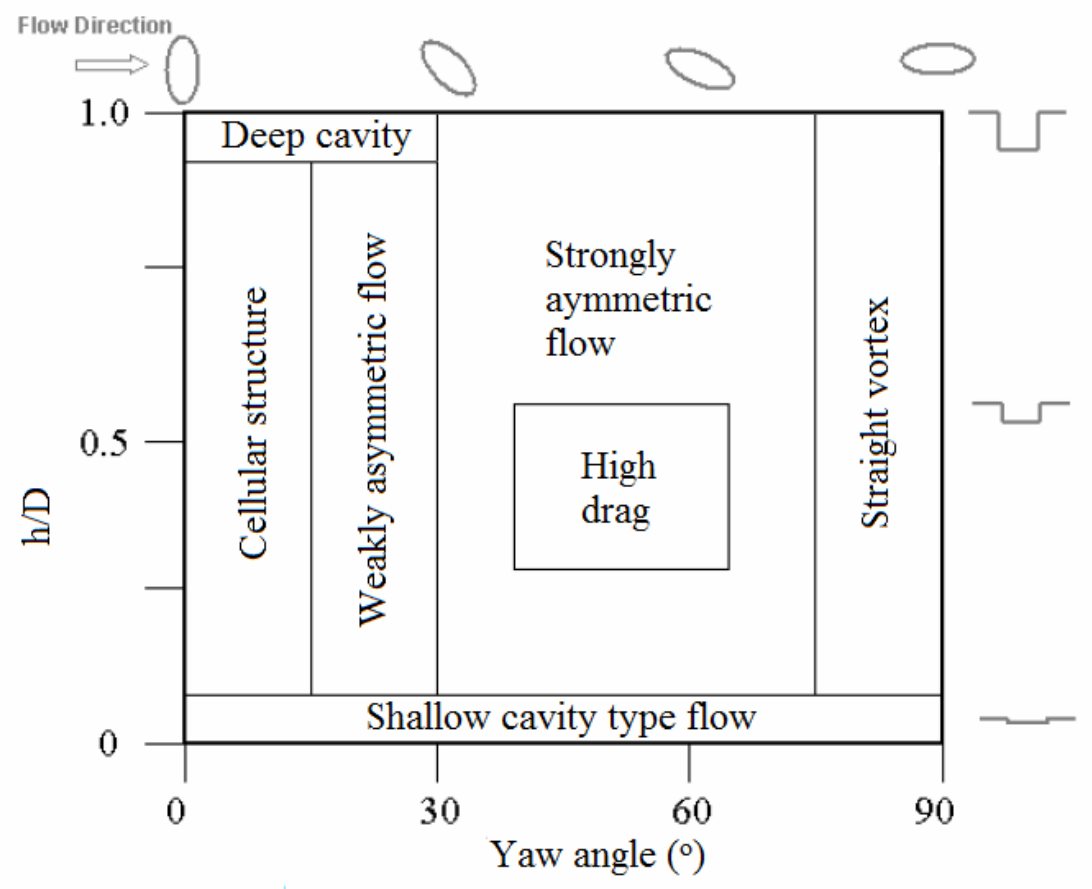

Figure 6-1 Modified classification of cavity flow regimes

\subsection{Recommendations for Future Work}

To extend and complement the findings of the present research, the following recommendations are made:

- In the experimental setup used in the present study, direct measurement of the flow in a major part of the separated shear layer was not possible, due to the limited optical access caused by the ground plate. Considering that the separated shear layer is the driving mechanism of the flow inside the cavity and contains the largest level of fluctuations, it is recommended to modify the experimental setup to make it possible to measure the flow in the shear layer and the flow in the cavity volume simultaneously. This may be achieved through modifications in the wind tunnel test model, or by using stereoscopic PIV technique in the case of shallow cavities.

- The deepest cavity studied in the present work is the cavity with $h / D=1.0$. Since previous studies involving nominally two-dimensional rectangular cavities have 
shown that further changes in the flow regime occur for larger $\mathrm{h} / \mathrm{D}$ ratios, it is recommended that to extend the present experiments to larger $\mathrm{h} / \mathrm{D}$ ratios, in order to investigate the changes in the flow regime for elliptical cavities.

- In the present study, the elliptical cavity is found to contain a two-cell structure in certain combinations of depth and yaw angle. As discussed in Chapter 2, formation of cellular structures in cavities in rectangular cavities depends on the width to depth ratio of the cavity, in such a way that a larger number of cells are formed in wider cavities. An investigation of the flow in elliptical cavities with larger aspect ratios is recommended to investigate this relationship in the case of cavities with elliptical planform.

- As mentioned in Chapter 2, cavity flows may include dynamic phenomena that occur at various frequencies, including frequencies considerably larger than those measurable with the measurement equipment used in the experiments of the present research. Moreover, the Proper Orthogonal Decomposition analysis used in the present research to study the amplitude of vortex oscillations, does not provide any information regarding frequency of the oscillations. To obtain information about the dynamic behaviour of the flow in the cavity in time domain, measurement and analysis techniques with the ability to resolve the timedependent behaviour of the flow are recommended. Examples of these techniques include hotwire anemometry, Laser Doppler Velocimetry, and high-speed Particle Image Velocimetry, combined Fourier analysis.

- Due to the computational resources required for unsteady simulations of the cavity flow for various combinations of depth and yaw angle, steady numerical simulations were used to analyze the cavity flow in the present study. Therefore, the results of the numerical simulations did not reflect possible time-dependent behaviour of the flow. To obtain information about cavity flow dynamics, unsteady numerical simulations with sufficiently small time steps are recommended. Such simulations will also complement the high temporal resolution measurement techniques suggested in the previous paragraph. 
- In the present study, a two-dimensional planar PIV technique was used. Therefore, in order to construct the three-dimensional flow structure in the cavity volume, measurements were carried out in multiple horizontal and vertical planes. Use of the recently developed volumetric and tomographic PIV techniques, which make it possible to simultaneously measure all three velocity components in a three-dimensional volume, is recommended for obtaining more complete and detailed information about the three-dimensional flow structure in the cavity volume. 


\section{REFERENCES}

[1] Marec, J. P., Drag reduction: A Major Task of Research, Notes of Numerical Fluid Mechanics - Aerodynamic Drag Reduction Technologies: Proceedings of the CEAS/DragNet European Drag Reduction Conference, Vol. 76, Potsdam, Germany, June 2000.

[2] Schneider, W., The Importance of Aerodynamics in Development of Commercially Successful Transport Aircraft, Notes of Numerical Fluid Mechanics - Aerodynamic Drag Reduction Technologies: Proceedings of the CEAS/DragNet European Drag Reduction Conference, Vol. 76, Potsdam, Germany, June 2000.

[3] Friesing, H., Measurement of the Drag Associated with Recessed Surfaces: Cutouts of Rectangular and Elliptical Planform, ZWBFB 628, 1936 (RAE Library Translation 1614, 1971).

[4] Roshko, A., Some Measurement of Flow in a Rectangular Cutout, NACA TN Report, No. 3488, 1955.

[5] Savory, E., Toy, N., The Flows Associated with Elliptical Cavities, Symposium on separated flows, ASME Fluids Engineering Conference, Washington, DC, FED Paper No. 149, pp. 95-103, 1993.

[6] Hering, T., Savory, E., Flow Regimes and Drag Characteristics of Elliptical Cavities with Varying Depth, Journal of Fluids Engineering, Transactions American Society of Mechanical Engineers, Vol. 129, pp. 1577-1583, 2007.

[7] Morton, B. R., The Generation and Decay of Vorticity, Geophysical and Astrophysical fluid dynamics, Vol. 28, pp. 277-308, 1984.

[8] Green, S. I., Fluid Vortices, Kluwer Academic Publishers, The Netherlands, ISBN: 0-7923-3376-4, 1995.

[9] Morton, B. R., Physical Appraisal of Some Classic Flows in Terms of Boundary Generated Vorticity, Proceedings of the $13^{\text {th }}$ Australasian Fluid Mechanics Conference, Melbourne, Australia, December 1998.

[10] Wu, J. Z., Wu, J. M., Boundary Vorticity Dynamics since Lighthill's 1963 Article: Review and Development, Theoretical and computational fluid dynamics, Vol. 10, pp. 459-474, 1998.

[11] Jeong, J., Hussain, F., On the Identification of a Vortex, Journal of Fluid Mechanics, Vol. 285, pp. 69-94, 1995.

[12] Tani, I., Iuchi, M., Komoda, H., Experimental Iinvestigation of Flow Separation Associated with a Step or Groove, Aeronautical Research Institute, University of Tokyo, Report No. 364, 1961.

[13] Charwat, A. F., Roos, J. N., Dewey, Jr., F. C., Hitz, J. A., Investigation of Separated Flows, Part I: The Pressure Field, Journal of Aerospace Science, Vol. 28 (6-7), pp. 457-470, 1961. 
[14] EDSU, Aerodynamics and Aero-Acoustics of Rectangular Planform Cavities. Part I: Time-Averaged Flow, Data Item 02008, 2004.

[15] Plentovich, E. B., Stallings R. L., Tracy, M. B., Experimental Cavity Pressure Measurements at Subsonic and Transonic Speeds, NASA-TP-3358, 1993.

[16] Maull, D. J., East L. F., Three dimensional Flow in Cavities, Journal of Fluid Mechanics, Vol. 16, pp. 620-632, 1963.

[17] Hunt, J. C. R., Abell, C. J., Peteka, J. A., Woo, H., Kinematical studies of the flows around free or surface-mounted obstacles; applying topology to flow visualization, Journal of Fluid Mechanics, Vol. 86, part 1, pp. 179-200, 1978.

[18] Plentovich, E. B., Three-Dimensional Cavity Flow Fields at Subsonic and Transonic Speeds, NASA TM 4209, 1990.

[19] Atvars, K., Knowles, K., Rotchie, S., A., Lawson, N. J., Experimental and Computational Investigation of an "Open" Transonic Cavity Flow, Proceedings of the Institution of Mechanical Engineers, Part G: Journal of Aerospace Engineering, Vol. 223, No. 4, pp. 357-368, April 2009.

[20] Ritchie, S. A., Lawson N. J., Knowles, K., An Experimental and Numerical Investigation of an Open Transonic Cavity, AIAA, $21^{\text {st }}$ Applied Aerodynamics Conference, Orlando, Florida, June 2003.

[21] Haigermoser, C., Scarano, F., Onorato, M., Investigation of the Flow in a Rectangular Cavity Using Tomographic and Time Resolved PIV, Proceedings of ICAS 2008: $26^{\text {th }}$ International Congress of the Aeronautical Sciences, Anchorage, Alaska, September 2008.

[22] Brown, G. L., Roshko, A., On Density Effects and Large Structure in Turbulent Mixing Layer, Journal of Fluid Mechanics, Vol. 64, Part 4, pp. 775-816, 1974.

[23] Ashcroft, G., Zhang, X., Vortical Structures over Rectangular Cavities at Low Speed, Physics of Fluids, Vol. 17, pp. 1-8 (015104), 2005.

[24] Haigermoser, C., Vesely, L., Novara, M., Onorato, M., A Time-Resolved Particle Image Velocimetry Investigation of a Cavity Flow with a Thick Incoming Turbulent Boundary Layer, Physics of Fluids, Vol. 20, pp. 1-14 (105101), 2008.

[25] Murray, N., Sällström, E., Ukeiley, L., Properties of Subsonic Open Cavity Flow Fields, Physics of Fluids, Vol. 21, pp. 1-16 (095103), 2009.

[26] Ukeiley, L., Murray, N., Velocity and Surface Pressure Measurements in an open Cavity, Experiments in Fluids, Vol. 38, pp. 656-671, 2005.

[27] Özsoy, E., Rambaud, P., Stitou, A., Riethmuller, M. L., Vortex Characteristics in Laminar Cavity Flow at Very Low Mach Number, Experiments in Fluids, Vol. 38, pp. 133-145, 2005.

[28] Manovski, P., Giacobello, M., Soria, J., Particle Image Velocimetry Measurements over an Aerodynamically Open Two-Dimensional Cavity, $16^{\text {th }}$ Australasian Fluid Mechanics Conference, Gold Coast, Australia, December 2007. 
[29] Ozalp, C., Pinarbasi, A., Sahin, B., Experimental Measurement of Flow Past Cavities of Different Shapes, Experimental Thermal and Fluid Science, Vol. 34, pp. 505-515, 2010.

[30] Cheun, B. S., Toy, N., Moss, W. D., The Effect of Upstream Boundary Layer Thickness upon Flow Past a Backward-Facing Step, $7^{\text {th }}$ Symposium on Turbulence, Rolla, MO, Proceedings (A83-49801 pp. 24-35), September 1981.

[31] Adams, E. W., Johnston, J. P., Effects of the Separating Shear Layer on the Reattachment Flow Structure - Part 1: Pressure and Turbulence Quantities, Experiments in Fluids, Vol. 6, pp. 400-408, 1988.

[32] Adams, E. W., Johnston, J. P., Effects of the Separating Shear Layer on the Reattachment Flow Structure - Part 2: Reattachment Length and Wall Shear Stress, Experiments in Fluids, Vol. 6, pp. 493-499, 1988.

[33] Hudy, L. M., Simultaneous Wall-Pressure and Velocity Measurements in the Flow Field Downstream of an Axisymmetric Backward Facing Step, PhD Dissertation, Michigan State University, 2005.

[34] Terekhov V. I., Bogatko, T. V., Effect of Boundary Layer Thickness before the Flow Separation on Aerodynamic Characteristics and Heat Transfer behind an Abrupt Expansion in a Round Tube, Thermophysics and Aeromechanics, Vol. 15, No. 1, pp. 91-97, 2008.

[35] Savory, E., Perret, L., Rivet, C., Modeling Considerations for Examining the Mean and Unsteady Flow in a Simple Urban-Type Street Canyon, Proceedings of ICWE13: $13^{\text {th }}$ International Conference on Wind Engineering, Amsterdam, The Netherlands, July 2011.

[36] Rockwell, D., Naudascher, E., Review - Self-Sustaining Oscillations of Flow Past Cavities, Journal of Fluids Engineering, Vol. 100, pp. 152-165, 1978.

[37] Lin, J. C., Rockwell, D., Organized Oscillations of Initially Turbulent Flow Past a Cavity, AIAA Journal, Vol. 39, No. 6, pp. 1139-1151, 2001.

[38] Rossiter, J. E., Wind Tunnel Experiment on the Flow in a Rectangular Cavities at Subsonic and Transonic Speeds, British ARC R\& M No. 3428, 1964.

[39] McGregor O. W., White R. A., Drag of Rectangular Cavities in Supersonic and Transonic Flow Including the Effects of Cavity Resonance, AIAA Journal, Vol. 8, No. 11, pp. 1959-1964, 1970.

[40] Block, P. J. W., Noise Response of Cavities of Varying Dimensions at Subsonic Speeds, NASA TN D-8351, 1976.

[41] Tam, C. K. W., Block, P. J. W., On the Tones and Pressure Oscillations Induced by Flow Over Rectangular Cavities, Journal of Fluid Mechanics, Vol. 89, pp. 373399, 1978.

[42] Sarohia, V., Experimental Investigation of Oscillations in Flows over Shallow Cavities, AIAA Journal, Vol. 15, No. 7, pp. 984-991, 1977. 
[43] Gharib, M., Roshko, A., The Effect of Flow Oscillations on Cavity Drag, Journal of Fluid Mechanics, Vol. 177, pp. 501-530, 1987.

[44] Ahuja, K. K., Mendoza, J., Effects of Cavity Dimensions, Boundary Layer, and Temperature on Cavity Noise with Emphasis on Bench-mark Data to Validate Computational Aeroacoustics Codes, NASA contractor report: final report contract NAS1-19061, 1995.

[45] Grace, S. M., Dewar, W. G., Wroblewski, D. E., Experimental Investigation of the Flow Characteristics within a Shallow Wall Cavity for Both Laminar and Turbulent Upstream Boundary Layers, Experiments in Fluids, Vol. 36, pp. 791804, 2004.

[46] Marsden, O., Jondeau, E., Souchotte, P., Bogey, C., Bailly, C., Juvé, D., Investigation of Flow Features and Acoustic Radiation of a Round Cavity, $14^{\text {th }}$ AIAA/CEAS Aeroacoustics Conference, Vancouver, Canada, May 2008.

[47] Wieghardt, K., Increase in Turbulent Frictional Resistance Caused by Surface Irregularities, MAP R\&T 103, Trans. of FB1563, 1946.

[48] Savory, E., Toy, N., Disimile, P. J., Dimicco, R. G., The Drag of ThreeDimensional Rectangular Cavities, Applied Scientific Research, Vol. 50, pp. 325346, 1993.

[49] Czech, M., The Acoustics and Aerodynamics of Turbulent Flow over Yawed Rectangular Cavities, University of Surrey, PhD Thesis, 2000.

[50] Czech, M., Savory, E., Toy, N., Mavrides, T., Flow Regimes Associated with Yawed Rectangular Cavities, The Aeronautical Journal, Vol. 105, pp.125-134, 2001.

[51] Hiwada, M., Kawamura, T., Mabuchi, I., Kumada, M., Some Characteristics of Flow Pattern and Heat Transfer Past a Circular Cylindrical Cavity, Bulletin of the JSME, Vol. 25, No. 220, pp. 1744-1752, 1983.

[52] Savory, E., Toy, N., Gaudet, L., Effect of Lip Configuration on the Drag of a Circular Cavity, Emerging Techniques In Drag Reduction, Mechanical Engineering Publications Ltd, pp. 317-335, 1996.

[53] Dybenko, J., Savory, E., An Experimental Investigation of Turbulent Boundary Layer Flow over Surface Mounted Circular Cavities, IMechE Journal of Aerospace Engineering, Vol. 222, pp. 109-125, 2008.

[54] Haigermoser, C., Scarano F., Onorato, M., Investigation of the Flow in a Circular Cavity Using Stereo and Tomographic Particle Image Velocimetry, Experiments in Fluids, Vol. 46, pp. 517-526, 2009.

[55] Haugen, R. L., Dhanak, A. M., Momentum Transfer in Turbulent Separated Flow Past a Rectangular Cavity, Journal of Applied Mechanics, Transactions American Society of Mechanical Engineers, Vol. 33, pp. 641-464, 1966.

[56] Gaudet, L., Winter, K. G., Measurements of the Drag of Some Characteristic Aircraft Excrescences Immersed in Turbulent Boundary Layers, Royal Aircraft Establishment, Technical Memorandum Aero, 1973. 
[57] Young, A. D., Paterson, J. H., Aircraft Excrescence Drag, NATO AGARD-AG264, J. L. Jones editor, 1981.

[58] Hering, T., Lift and Drag of Yawed Elliptical Cavities with Varying Depths, University of Western Ontario, Master of Engineering Science Thesis, 2006.

[59] Armaly, B.F., Durst, F., Pereira J. C. F., Schönung B., Experimental and Theoretical Investigation of Backward-Facing Step Flow, Journal of Fluid Mechanics, Vol. 127, pp. 473-496, 1983.

[60] Williams, P. T., Baker, A. J., Numerical Simulations of Laminar Flow over a 3D Backward Facing Step, International Journal for Numerical Methods in Fluids, Vol. 24, pp. 1159-1183, 1997.

[61] Shih, C., Ho, C. M., Three-dimensional Recirculation Flow in a Backward Facing Step, Journal of Fluids Engineering, Transactions American Society of Mechanical Engineers, Vol. 116, pp.228-232, 2004.

[62] Le, H., Moin, P., Kim, J., Direct Numerical Simulation of Turbulent Flow over a Backward-Facing Step, Journal of Fluid Mechanics, Vol. 330, pp. 349-374, 1997.

[63] Wengle, H., Huppertz, A., Bärwolff, G., Janke, G., The Manipulated Transitional Backward-Facing Step Flow: An Experimental and Direct Numerical Simulation Investigation, European Journal of Mechanics-B, Vol. 20, pp. 25-46, 2001.

[64] Yao, H., Cooper, R. K., Raghunathan, S., Numerical Simulation of Incompressible Laminar Flow over Three-Dimensional Rectangular Cavities, Journal of Fluids Engineering, Transactions American Society of Mechanical Engineers, Vol. 126, pp. 919-927, 2004.

[65] Sinha, S. N., Gupta, A. K., Oberai, M. M., Laminar Separating Flow over Backsteps and Cavities-Part II: Cavities, AIAA Journal, Vol. 20, pp. 370-375, 1982.

[66] Fuglsang, D. F., Cain, A.B., Evaluation of Shear Layer Cavity Resonance Mechanisms by Numerical Simulation, AIAA Paper, $30^{\text {th }}$ Aerospace Sciences Meeting and Exhibit, United States, 1992.

[67] Rowley, C. W., Colonius, T., Basu, A., On Self-Sustained Oscillations in TwoDimensional Compressible Flow over Rectangular Cavities, Journal of Fluid Mechanics, Vol. 455, pp. 315-346, 2002.

[68] Rizzetta, D. P., Numerical Simulation of Supersonic Flow over a ThreeDimensional Cavity, AIAA Journal, Vol. 26, pp. 799-807, 1988.

[69] Rizzetta, D. P., Visbal, M. R., Large-Eddy Simulation of Supersonic Cavity Flowfields Including Flow Control, AIAA Journal, Vol. 41, pp. 1452-1462, 2003.

[70] Grottadaurea, M., Rona, A. The Radiating Pressure Field of a Turbulent Cylindrical Cavity Flow, 14 ${ }^{\text {th }}$ AIAA/CEAS Aeroacoustics Conference, Vancouver, Canada, May 2008.

[71] Zdanski, P. S. B., Ortega, M. A., Fico Jr., N. G. C. R., Numerical Study of the Flow over Shallow Cavities, Computers and Fluids, Vol. 32, pp. 953-974, 2003. 
[72] Durteste, S., Analysis of Cavity Drag Using Glasgow University Flow Code, University of Glasgow, Final year project, 2001.

[73] Hering, T., Dybenko, J., Savory, E., Experimental Verification of CFD Modeling of Turbulent Flow over Circular Cavities, Canadian Society for Mechanical Engineering Forum, Kananaskis, Canada, 2006.

[74] Marsden, Bogey, C., Bailly, C., Numerical Investigation of Flow Features and Acoustic Radiation from Round Cavities, $5^{\text {th }}$ European Conference on Computational Fluid Dynamics, Lisbon, Portugal, June 2010.

[75] FLUENT, FLUENT 6.3 user's guide, FLUENT Inc. 2006.

[76] Ferziger, J. H., Perić, M., Computational Methods for Fluid Dynamics, SpringerVerlag, Berlin, 1996.

[77] Spalart, P., Allmaras, S., A One-Equation Turbulence Model for Aerodynamic Flows, Technical Report AIAA-92-0439, American Institute of Aeronautics and Astronautics, No. 1, pp. 5-21, 1992.

[78] Shih, T. H., Liou, W. W., Shabbir, A., Yang, Z., Zhu. J., A New k- $\varepsilon$ EddyViscosity Model for High Reynolds Number Turbulent Flows - Model Development and Validation, Computers Fluids, Vol. 24(3), pp. 227-238, 1995.

[79] Wilcox, D. C., Turbulence Modeling for CFD, DCW Industries, Inc., La Canada, California, 1998.

[80] Daly, B. J., Harlow, F. H., Transport Equations in Turbulence, Physics of Fluids, Vol. 13, pp. 2634-2649, 1970.

[81] Lien, F. S., Leschziner, M. A., Assessment of Turbulent Transport Models Including Non-Linear RNG Eddy-Viscosity Formulation and Second-Moment Closure, Computers and Fluids, Vol. 23, No. 8, pp. 983-1004, 1994.

[82] Kays, W., Crawford, M. E., Weigand, B., Convective Heat and Mass Transfer, $4^{\text {th }}$ Edition, McGraw-Hill, 2005.

[83] Launder, B. E., Second-Moment Closure and Its Use in Modeling Turbulent Industrial Flows. International Journal for Numerical Methods in Fluids, Vol. 9, pp. 963-985, 1989.

[84] Launder, B. E., Second-Moment Closure: Present... and Future?, International Journal of Heat and Fluid Flow, Vol. 10, No. 4, pp. 282-300, 1989.

[85] White, F.M., Fluid Mechanics, $6^{\text {th }}$ Edition, McGraw-Hill, 2006.

[86] Schlichting, H., Gersten, K., Boundary Layer Theory, $8^{\text {th }}$ Edition, SpringerVerlag, Berlin, 2000.

[87] Young, A. D., Boundary Layers, BSP professional books, Blackwell, Oxford, 1989.

[88] Kim, S. E., Choudhury D., A Near-Wall Treatment Using Wall Functions Sensitized to Pressure Gradient, ASME FED Separated and Complex Flows, ASME, Vol. 217, pp. 273-279, 1995. 
[89] Chen, H. C., Patel, V. C., Near-Wall Turbulence Models for Complex Flows Including Separation, AIAA Journal, Vol. 26, No. 6, pp. 641-648, 1988.

[90] Tennekes, H., Lumley, J. L., A First Course in Turbulence, MIT Press, 1976.

[91] Versteeg, H. K., Malalasekra, W., An Introduction to Computational Fluid Dynamics, $2^{\text {nd }}$ Edition, Pearson Education Ltd., 2007.

[92] Bailey, S. C. C., The effect of Wall Proximity on Vortex Shedding from a Square Cylinder, University of Western Ontario, Master of Engineering Science Thesis, 2001.

[93] Narasimha, R., Prasad, S. N., Leading Edge Shape for Flat Plate Boundary Layer Studies, Experiments in Fluids, Vol. 17, No. 5, pp. 358-360, 1994.

[94] Saric, W. S., Design of High-Reynolds-Number Flat-Plate Experiments in the NTF, NASA-CR-184627, 1988.

[95] Lee, S. L., Ballesteros, P., Uniformly Loaded Rectangular Plate Supported at the Corners, International Journal of Mechanical Science, Pergamon press Ltd., Vol. 2, pp. 206-211, 1960.

[96] Saric, W. S., Influence of High-Amplitude Noise on Boundary-Layer Transition to Turbulence, Final Technical Report, AFOSR, F49620-96-1-0369, 1998.

[97] Westin, K. J. A., Boiko, A. V., Klingmann, B. G. B., Kozlov V. V., Alfredsson, P. H., Experiments in a Boundary Layer Subjected to Free Stream Turbulence. Part 1. Boundary Layer Structure and Receptivity, Journal of Fluid Mechanics, Vol. 281, pp. 193-218, 1994.

[98] Fransson, J. H. M., Brandt, L., Talamelli, A., Cossu, C., Experimental Study of the Stabilization of Tollmien-Schlichting Waves by Finite Amplitude Streaks, Physics of Fluids, Vol. 17, pp. 1-15 (054110), 2005.

[99] AbuOmar, M. M., Experimental Study of the Flow around Surface-Mounted Pyramids, University of Western Ontario, PhD Thesis, 2002.

[100] Larichkin, V. V., Yakovenko, S. N., Effect of Boundary-Layer Thickness on the Structure of a Near-Wall Flow with a Two-Dimensional Obstacle, Journal of Applied Mechanics and Technical Physics, Vol. 44, No. 3, pp. 365-372, 2003.

[101] Ruderich, R., Fernholz, H. H., An Experimental Investigation of a Turbulent Shear Flow with Separation, Reverse Flow, and Reattachment, Journal of Fluid Mechanics, Vol. 163, pp. 283-322, 1986.

[102] Okamoto, S., Turbulent Shear Flow behind Rows of Square Plates Placed on a Plane Boundary, Atmospheric Environment, Vol. 20, No. 8, pp. 1537-1546, 1986.

[103] Counihan, J., Hunt, J. C. R., Jackson, P. S., Wakes behind Two-Dimensional Surface Obstacles in Turbulent Boundary Layers, Journal of Fluid Mechanics, Vol. 64, Part 3, pp. 529-563, 1974.

[104] Naghib, H. N., Chauhan, K., A., Monkewitz, P. A., Approach to an Asymptotic State for Zero Pressure Gradient Turbulent Boundary Layers, Philosophical Transactions of Royal Society, Vol. 365, pp.755-770, 2007. 
[105] Wu, X., Moin, P., Direct Numerical Simulation of Turbulence in a Nominally Zero-Pressure-Gradient Flat-Plate Boundary Layer, Journal of Fluid Mechanics, Vol. 630, pp. 5-41, 2009.

[106] Raffel, M., Willert, C., Kompenhans, J., Particle Image Velocimetry: A Practical Guide, Springer-Verlag, Berlin, 1998.

[107] Loth, E., Numerical Approaches for Motion of Dispersed Particles, Droplets, and Bubbles, Progress in Energy and Combustion Science, Vol. 26, pp. 161-223, 2000 .

[108] Piirto, M., Saarenrinne., P., Eloranta, H., Karvinen, R., Measuring Turbulence Energy with PIV in a Backward Facing Step Flow, Experiments in Fluids, Vol. 35, pp. 219-236, 2003.

[109] Dybenko, J., An Experimental Investigation of Turbulent Boundary Layer Flow over Surface Mounted Circular Cavities. University of Western Ontario, Master of Engineering Science Thesis, 2005.

[110] Wheeler, A., Ganji, A., Introduction to Engineering Experimentation, $2^{\text {nd }}$ Edition, Prentice Hall Inc., NJ., 1996.

[111] Cower, E., A., Monismith, S. G., A hybrid Digital Tracking Velocimetry Technique, Experiments in Fluids, Vol. 22, pp. 199-211, 1997.

[112] Christensen, K. T., The Influence of Peak-Locking Errors on Turbulence Statistics Computed from PIV Ensembles, Experiments in Fluids, Vol. 36, pp. 484-497, 2004.

[113] Westerweel, J., Fundamentals of Digital Particle Image Velocimetry, Measurement Science and Technology, Vol. 8, pp. 1379-1392, 1997.

[114] Hamburg, M., Statistical Analysis for Decision Making, Harcourt, Brace \& World, 1970.

[115] Benedict, L. H., Gould, R. D., Towards Better Uncertainty Estimates for Turbulence Statistics, Experiments in Fluids, Vol. 22, pp. 129-136, 1996.

[116] Holmes, P., Lumley, J. L., Berkooz, G., Turbulence, Coherent Structures, Dynamical Systems, and Symmetry, Cambridge University Press, 1996.

[117] Smith T. R., Moehlis, J., Holmes, P., Low-Dimensional Modeling of Turbulence Using the Proper Orthogonal Decomposition: A Tutorial, Non-linear Dynamics, Vol. 41, pp. 275-307, 2005.

[118] Shlens, J., A Tutorial on Principal Component Analysis, Salk Institute of Biological Studies, US, 2009.

[119] Henderson, J., Badcock, K. J., Richards, B. E., Subsonic and Supersonic Transitional Cavity Flows, AIAA paper 2000-1966, 2000.

[120] Sherry, M., Lo Jacono, D., Sheridan, J., An Experimental Investigation of the Recirculation Zone Formed Downstream of a Forward Facing Step, Journal of Wind Engineering and Experimental Aerodynamics, Vol. 98, pp.888-894, 2010. 
[121] Perrin, R., Braza, M., Cid, E., Cazin, S., Barthet, A., Sevrain, A., Mockett, C., Thiele, F., Obtaining Phase Averaged Turbulence Properties in the Near Wake of a Circular Cylinder Using POD, Experiments in Fluids, Vol. 43, pp. 341-355, 2007.

[122] Kang, W., Lee, S. B., Sung, H. J., Self-Sustained Oscillations of Turbulent Flows over an Open Cavity, Experiments in Fluids, Vol. 45, pp. 693-702, 2008.

[123] Radcliffe, S. A., Two-dimensional CFD Investigation of the Drag of Rectangular Planform Cavities in a Flat Plate with Turbulent Boundary Layer, Final year project report, University of Glasgow, 2002. 


\section{Appendix A: Cavity Model Drawings}

All dimensions are in meters.

- Base plate
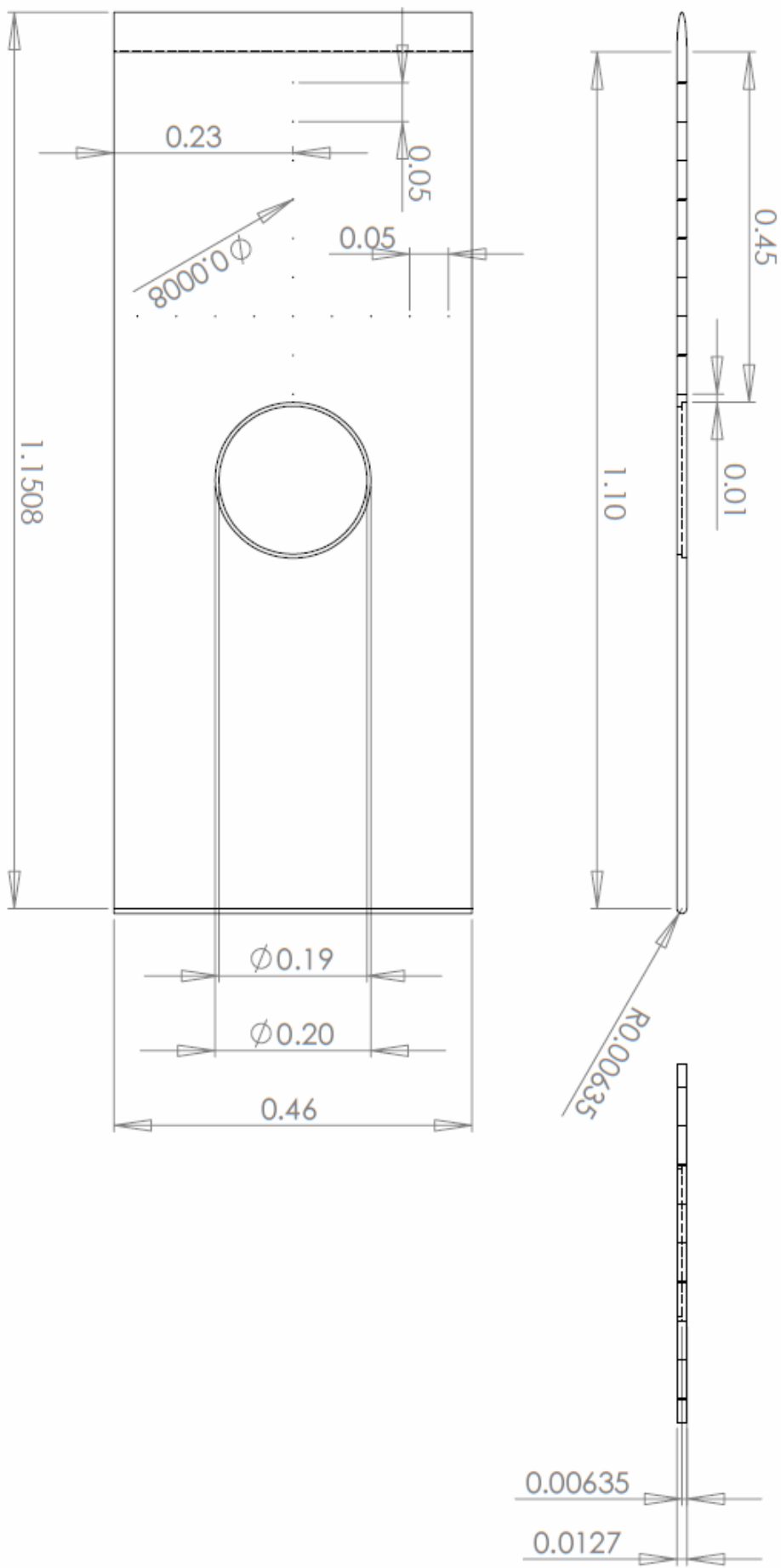
- Flap
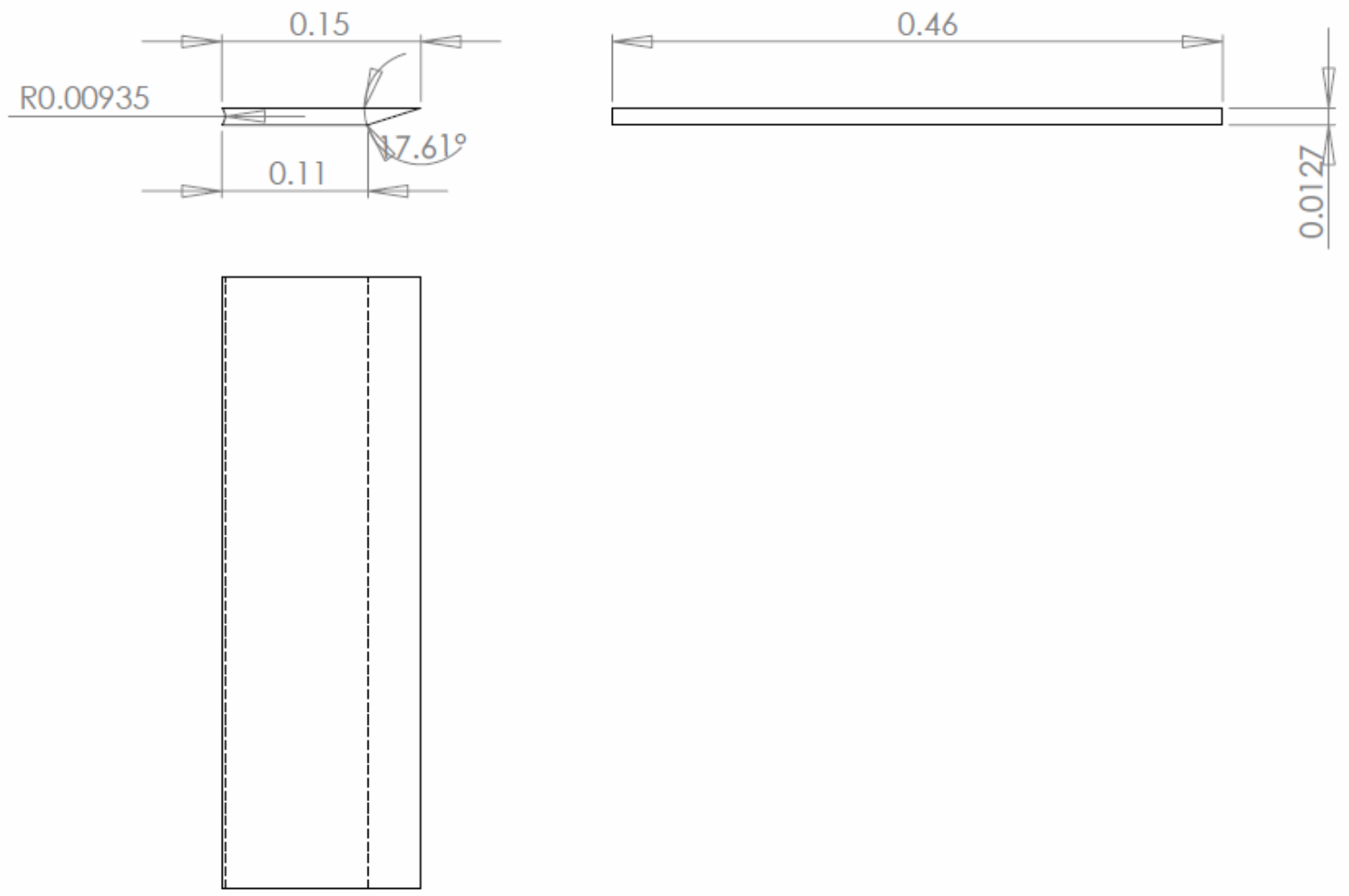

- Ground plate
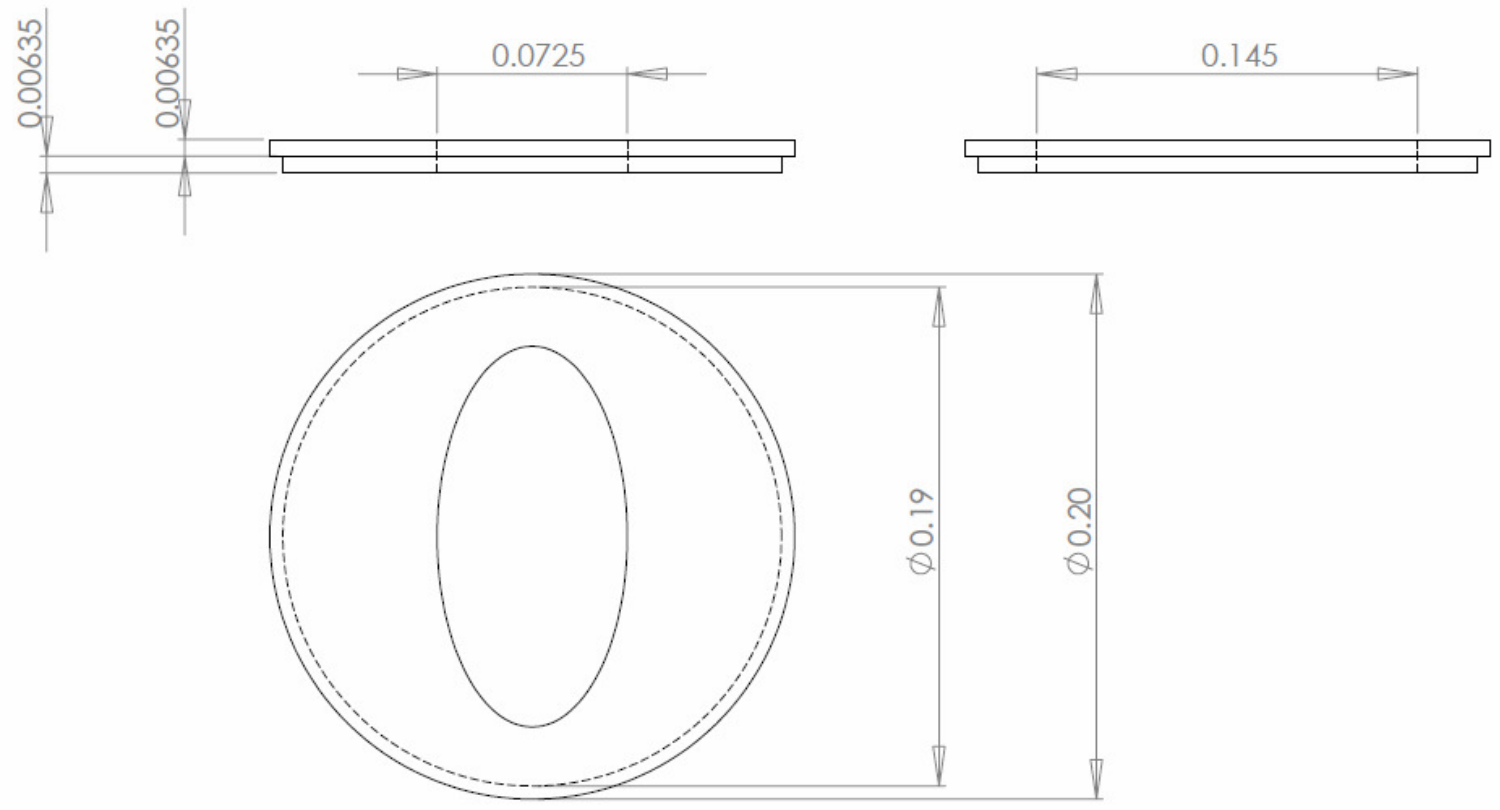
- Cavity walls
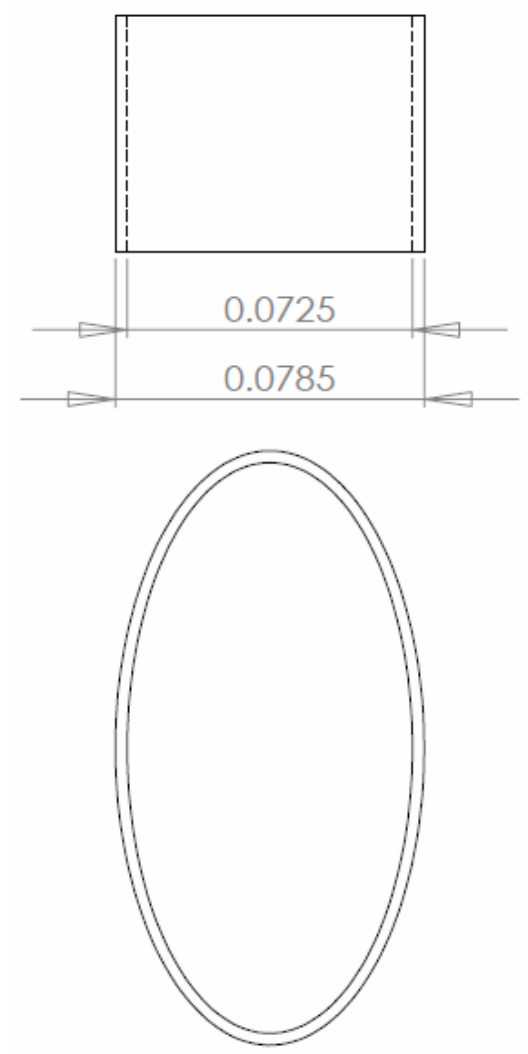

- Cavity base
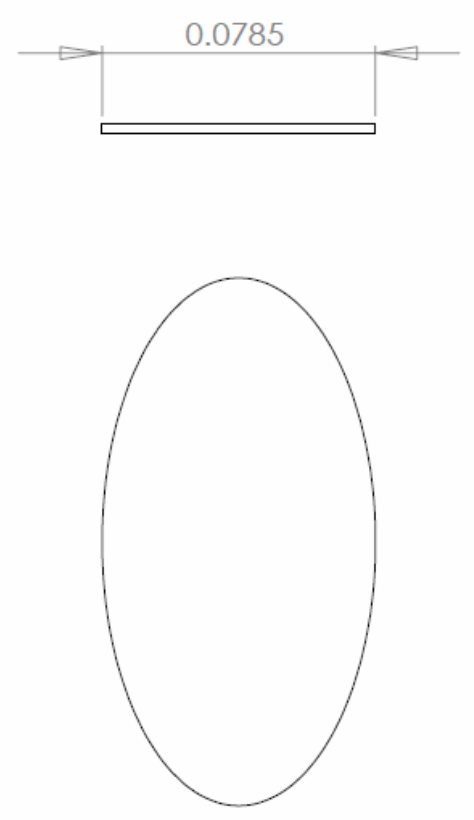
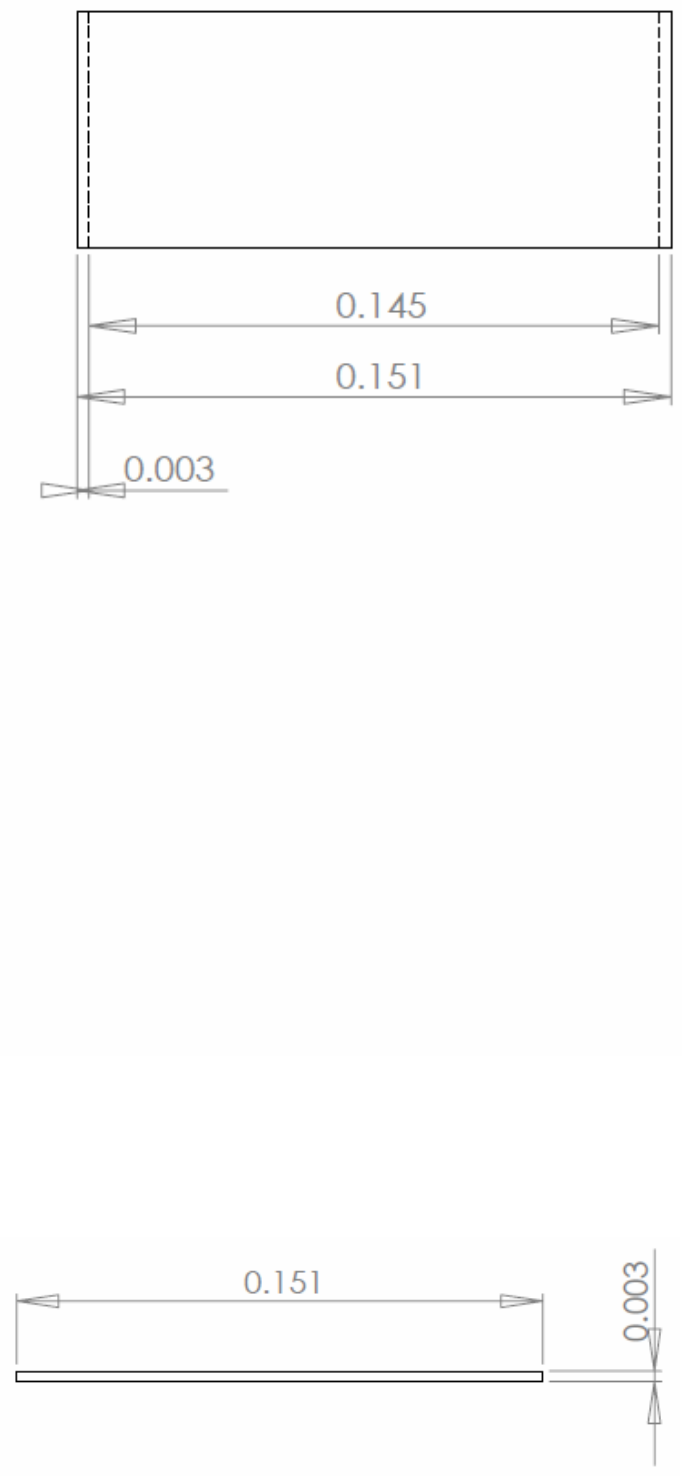
- Support leg
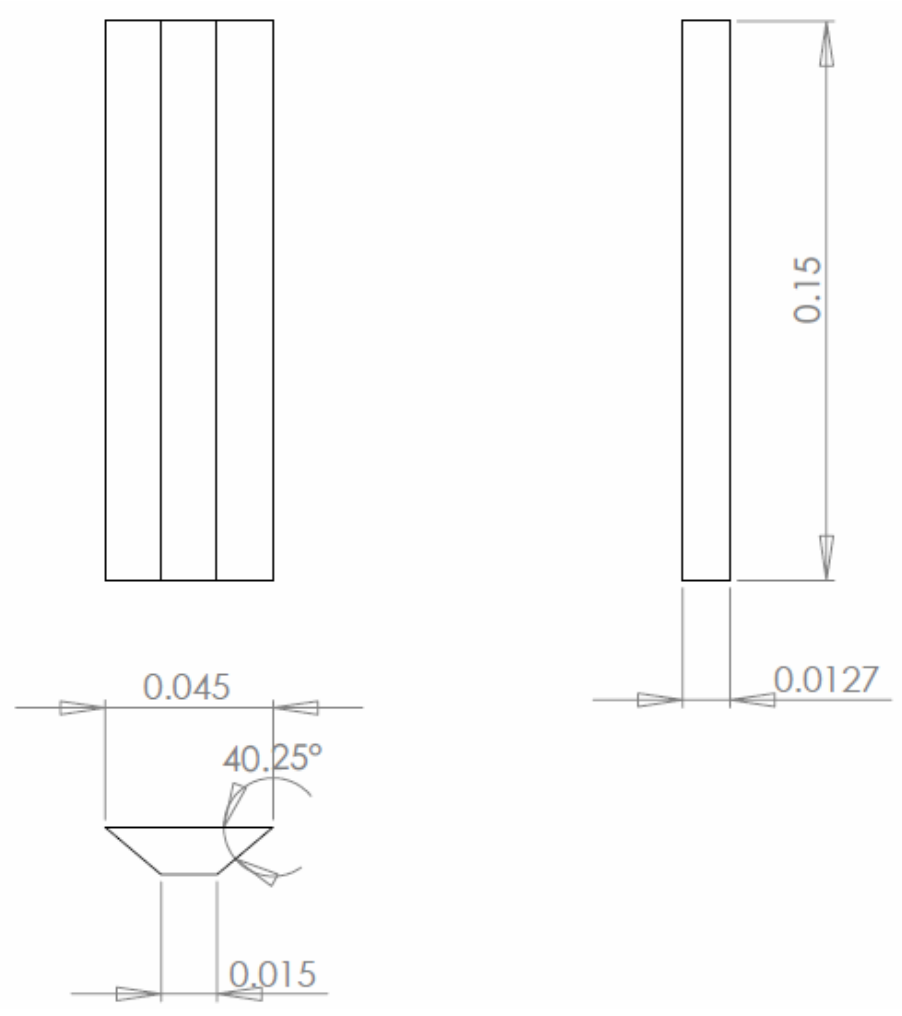

- Assembly

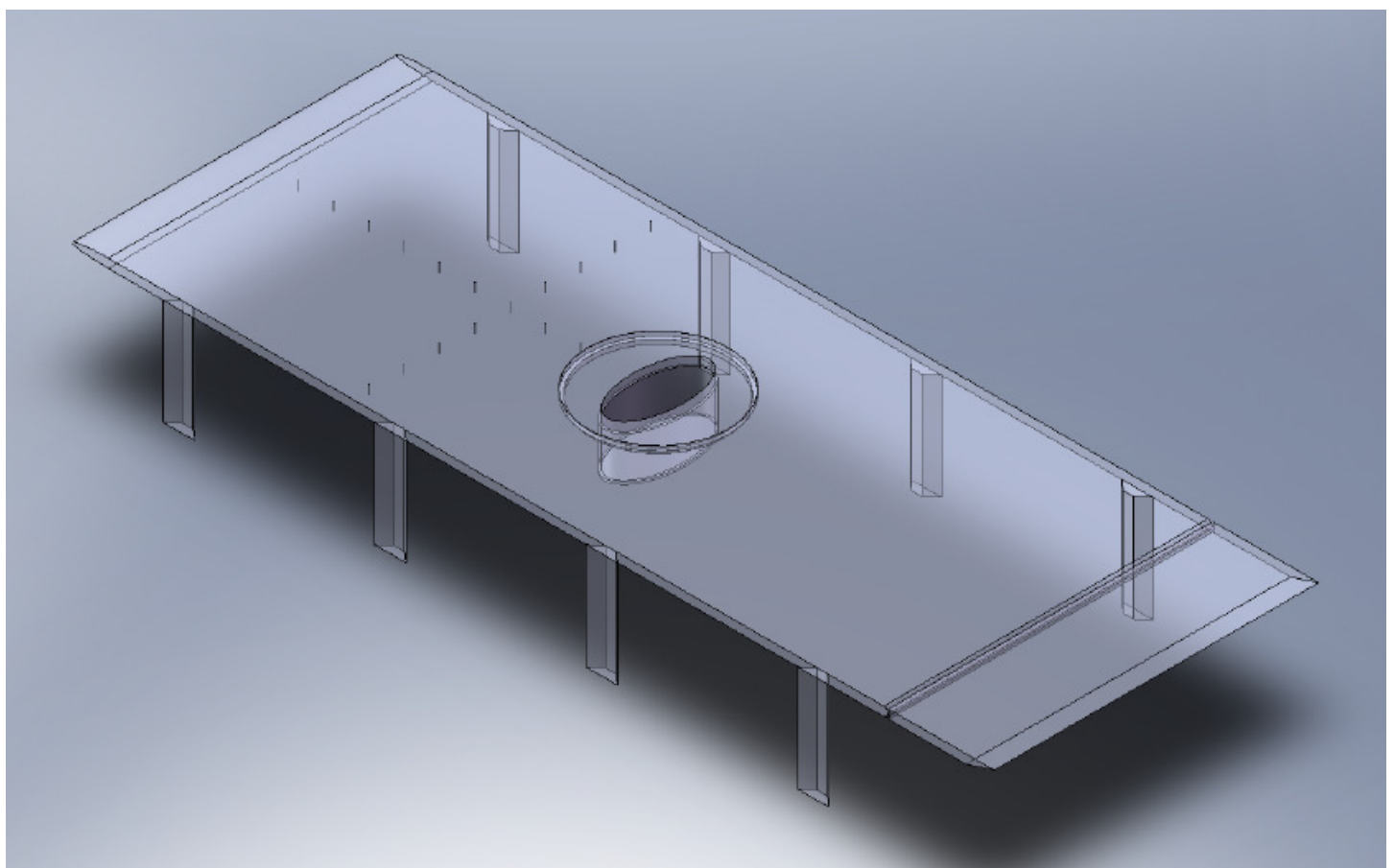




\title{
Curriculum Vitae
}

NAME

EDUCATION

1999-2001

1994-1999

Taravat Khadivi

\author{
M. Sc., Aerospace Engineering (Aerodynamics) \\ Sharif University of Technology, Tehran, Iran \\ B. Sc., Mechanical Engineering (Aerospace) \\ Sharif University of Technology, Tehran, Iran
}

HONORS \& AWARDS

2010

2009

Nominated for Graduate Student Teaching Award

The Best Teaching Assistant Award at MME

\section{PROFESSIONAL BACKGROUND}

2007- 2012

Department of Mechanical and Materials Engineering, The University of Western Ontario, London, Ontario, Canada

$\mathrm{PhD}$ candidate, research and teaching assistant

2003-2006

Safiran-Dnata Airport Service Co., Tehran, Iran

2000-2002

Technical and engineering manager

Aerospace Research Institute, Ministry of Science, Research and

Technology, Tehran, Iran

Research engineer

\section{JOURNAL PUBLICATIONS}

1. M. R. Soltani, T. Khadivi, A. Abbasi Hoseini, "Fluid Flow Study over the Wing of a Fighter-type Aircraft Model", International Journal of Engineering, Transactions B: Applications (ISSN: 1025-2495), Vol. 17, No. 4, pp. 399-410, Dec. 2004

\section{CONFERENCE PRESENTAIONS}

1. T. Khadivi, E. Savory, "Numerical Analysis of Flow around Low Aspect Ratio Cavities with Elliptical and Rectangular Planforms", CFD Society of Canada, Kanata, Canada, May 2009.

2. T. Khadivi, E. Savory, "Numerical Simulation of Flow over Low Aspect Ratio Elliptical Cavities, Western Research Forum, University of Western Ontario, London, Canada, February 2009.

3. T. Khadivi, E. Savory, CFD modeling of Flow over Elliptical Cavities with Varying Depths, Canadian Society for Mechanical Engineering Forum, Ottawa, Canada, June 2008.

4. A. Nejat, T. Khadivi, A. Naghib Lahouti, Effects of Nose Shape of Strap-on Boosters on Aerodynamic Characteristics of a Satellite Launch Vehicle with Strap- 
on Boosters, The 4th National Conference of Iranian Aerospace Society, Tehran Polytechnic, February 2003.

5. M. R. Soltani, T. Khadivi, A. R., Davari, Flow Control over the Wing of a Fightertype Configuration Using Boundary Layer Suction, International Council of the Aeronautical Sciences Congress, Toronto, Canada, September 2002.

6. T. Khadivi, A. Naghib Lahouti, A. Nejat, Parametric Analysis of Aerodynamic Characteristics of Launch Vehicles with Strap-on Boosters, International Council of the Aeronautical Sciences Congress, Toronto, Canada, September 2002.

7. M. R. Soltani, T. Khadivi, A. R., Davari, Flow Field Study over the Wing of a High Angle-of-attack Research Vehicle (HARV) Model, The $9^{\text {th }}$ Asian Congress of Fluid Mechanics, Esfehan, Iran, May 2002.

8. M. R. Soltani, T. Khadivi, The Effects of Suction on the Pressure Distribution over the Wing of a Highly Maneuverable Aircraft, The $10^{\text {th }}$ Annual Conference of Iranian Society of Mechanical Engineers, Khajeh Nasir Toosi University of Technology, Tehran, Iran, May 2002. 